\title{
The Plancherel decomposition for a reductive symmetric space I. Spherical functions
}

E. P. van den Ban and H. Schlichtkrull

\begin{abstract}
We prove the Plancherel formula for spherical Schwartz functions on a reductive symmetric space. Our starting point is an inversion formula for spherical smooth compactly supported functions. The latter formula was earlier obtained from the most continuous part of the Plancherel formula by means of a residue calculus. In the course of the present paper we also obtain new proofs of the uniform tempered estimates for normalized Eisenstein integrals and of the Maass-Selberg relations satisfied by the associated C-functions.
\end{abstract}

\section{Contents}

1 Introduction 2

\begin{tabular}{lll}
\hline 2 & Notation and preliminaries & 9
\end{tabular}

3 Wevl groups 13

4 Laurent functionals and operators 16

5 Normalization of residues and measures 19

6 A vanishing theorem 22

\begin{tabular}{lll}
\hline & Meromorphy of asvmptotic expansions & 27
\end{tabular}

8 Fourier inversion 29

$9 \quad$ The generalized Eisenstein integral 35

10 Temperedness of the Eisenstein integral 41

11 Initial uniform estimates 45

12 Svmmetric pairs of residue tvpe 51 
$\begin{array}{ll}15 \text { Uniform tempered estimates } & 68\end{array}$

16 Infinitesimal characters of the discrete series $\quad 73$

\begin{tabular}{|ll}
17 The constant term of the Eisenstein integral & 77
\end{tabular}

18 The Maass-Selberg relations $\quad 84$

19 The spherical Fourier transform $\quad 89$

20 The wave packet transform $\quad 92$

21 Fourier inversion for Schwartz functions 94

22 Properties of the Fourier transforms $\quad 98$

23 The spherical Plancherel theorem 104

\section{Introduction}

In this paper and its sequel [15] we determine the Plancherel decomposition for a reductive symmetric space $\mathrm{X}=G / H$. Here $G$ is a real reductive Lie group of Harish-Chandra's class and $H$ is an open subgroup of the group $G^{\sigma}$ of fixed points for an involution $\sigma$ of $G$. In the present paper we establish the Plancherel formula for $K$-finite (spherical) Schwartz functions on $X$, with $K$ a $\sigma$ invariant maximal compact subgroup of $G$. In [15] we shall derive the Plancherel decomposition, in the sense of representation theory, from it.

The space X carries a $G$-invariant measure $d x$; accordingly the regular representation $L$ of $G$ in $L^{2}(\mathrm{X})$ is unitary. The Plancherel decomposition amounts to an explicit decomposition of $L$ as a direct integral of irreducible unitary representations. The reductive group $G$ is a symmetric space of its own right, for the left times right action of $G \times G$. In this 'case of the group', the explicit Plancherel decomposition was obtained in the sixties and early seventies in the work of Harish-Chandra, see [30, [31, 32. His ideas, in particular those on the role of Eisenstein integrals and the Maass-Selberg relations satisfied by them, have been a major influence in our work. On the other hand, our approach to the Plancherel formula is via a residue calculus, and thus in a sense closer in spirit to the work of R.P. Langlands on the spectral decomposition in the theory of automorphic forms, see [37.

The results of this paper and [15] were found and announced in the fall of 1995 when both authors were visitors of the Mittag-Leffler Institute in Djursholm, Sweden. At the same time P. Delorme announced his proof of the Plancherel theorem. His results have appeared in a series of papers, partly 
in collaboration with J. Carmona, [19], 23], 24]. At the time of the announcement we relied on the theorem of Carmona and Delorme on the Maass-Selberg relations for Eisenstein integrals, 19, Thm. 2, which in turn relied on Delorme's paper [23]. On the other hand, we also announced the proof of a Paley-Wiener theorem for reductive symmetric spaces, generalizing Arthur's theorem [1] for the case of the group. The proof of the Paley-Wiener theorem has now appeared in [16, which is independent of the present paper and [15]. The present paper as well as [15] and [16] rely on [12] and [14].

Since the time of announcement we have been able to derive the MaassSelberg relations from those associated with the most continuous part of the decomposition; these had been obtained earlier in [5, 6 . The resulting proof of the Plancherel theorem in the present paper and [15] is independent of the one in 24]; moreover, it follows a completely different approach. Finally, we mention that T. Oshima has announced a Plancherel formula in [39], p. 604, but the details have not appeared. For a more extensive survey of recent developments, we refer the reader to $[13$.

For Riemannian symmetric spaces, the Plancherel formula is due to HarishChandra 27] and 28], p. 48. Later, it became incorporated in the above mentioned formula for the group. For further results in harmonic analysis on Riemannian symmetric spaces, we refer the reader to the references given in [35].

For reductive symmetric spaces of type $G_{\mathbb{C}} / G_{\mathbb{R}}$, the Plancherel formula is due to P. Harinck, [26]. Earlier, the Plancherel decomposition had been determined for specific non-Riemannian spaces of rank one, by V.F. Molchanov, J. Faraut, G. van Dijk and others; see 33], p. 185, for references.

We first give a rough outline of the contents of this paper and its sequel [15]. The following global picture should be kept in mind. We first concentrate on the Plancherel formula for $K$-finite functions, with $K \subset G$ a maximal compact subgroup that is chosen to be $\sigma$-stable. The latter condition is equivalent to the condition that the Cartan involution $\theta$ determined by $K$ commutes with $\sigma$. The building blocks of the formula will be discrete series representations of $\mathrm{X}$ and generalized principal series of the form $\operatorname{Ind}_{P}^{G}(\xi \otimes \nu \otimes 1)$, with $P=M_{P} A_{P} N_{P}$ a $\sigma \theta$-stable parabolic subgroup of $G$ with the indicated Langlands decomposition, $\xi$ a discrete series representation of $\mathrm{X}_{P}:=M_{P} / M_{P} \cap H$ and $\nu$ contained in the space $i \mathfrak{a}_{P \mathrm{q}}^{*}$ of unitary characters of $A_{P} / A_{P} \cap H$. For purposes of exposition this introduction is written under the simplifying assumption that the number of open $H$-orbits on $P \backslash G$ is one. In general the open orbits are parametrized by a finite set ${ }^{P} \mathcal{W}$ of representatives, and then one should take for $\xi$ the discrete series representations of all the spaces $\mathrm{X}_{P, v}:=M_{P} / M_{P} \cap v H v^{-1}, v \in{ }^{P} \mathcal{W}$.

In 10] we obtained the most continuous part of the Plancherel decomposition; this is the part built up from representations obtained by induction from a minimal $\sigma \theta$-stable parabolic subgroup $P_{0}=M_{0} A_{0} N_{0}$. Here $M_{0} / M_{0} \cap H$ is compact, so the theory of the discrete series did not critically enter at this stage. On the level of $K$-finite functions the most continuous part of the formula is described via a Fourier transform $\mathcal{F}_{0}$, which in turn is defined in terms of Eisenstein integrals $E^{\circ}\left(P_{0}: \lambda\right)$. The latter are essentially matrix coefficients of the principal series induced from $P_{0}$ and behave finitely under the action of the 
algebra $\mathbb{D}(\mathrm{X})$ of invariant differential operators on $\mathrm{X}$.

From the most continuous part of the Plancherel decomposition we derived in 12 a Fourier inversion formula for functions in $C_{c}^{\infty}(\mathrm{X}: \tau)$, the space of smooth compactly supported $\tau$-spherical functions on X, with $\tau$ a finite dimensional unitary representation of $K$. This formula expresses a function $f$ in terms of the meromorphic extension of $\mathcal{F}_{0} f$ to the complexification of $i \mathfrak{a}_{P_{0} q}^{*}$.

The strategy of the present paper is to put the inversion formula in a form that makes it valid for functions in the Schwartz space $\mathcal{C}(\mathrm{X}: \tau)$. This requires the introduction of Eisenstein integrals $E^{\circ}(P: \nu)$, for imaginary $\nu \in i \mathfrak{a}_{P \mathrm{q}}^{*}$, via residues of the Eisenstein integrals $E^{\circ}\left(P_{0}: \cdot\right)$. To show that these residual Eisenstein integrals define Fourier transforms on the Schwartz space we need the Maass-Selberg relations. It is here that the theory of the discrete series, initiated by M. Flensted-Jensen in 25] and further developed by T. Oshima and T. Matsuki in [40, enters. In our proofs we do not need the full classification of the discrete series. However, for the theory of the constant term developed in [17] to apply, both the necessity of the rank condition and the fact that the infinitesimal $\mathbb{D}(\mathrm{X})$-characters of discrete series representations are real and regular (see Theorem [16.1] due to [40]), play a crucial role.

The resulting inversion formula for Schwartz functions is called the spherical Plancherel formula, see Theorems 21.2 and 23.1. It naturally leads to the spherical Plancherel formula for $L^{2}$-functions, Theorem 23.4 The present paper finishes at this point, where it is not yet clear that the residual Eisenstein integrals are related to induced representations. This fact will be established in the second paper [15] by using the vanishing theorem of [14]. The contributions of all $K$-types can then be collected and lead to the representation theoretic Plancherel formula. At the end of the second paper it will also be shown that the residual Eisenstein integrals $E^{\circ}(P: \nu)$ equal the normalized Eisenstein integrals introduced in Delorme's paper [22. The idea is to use the automatic continuity theorem of W. Casselman and N.R. Wallach (20, [43]) to show that the residual Eisenstein integrals are matrix coefficients. An asymptotic analysis then completes the identification.

We shall now give a more detailed outline of the present paper. The first few sections concern preliminaries. In particular, in Section 5 we specify the normalizations of the residual operators and the measures used in the rest of the paper. In Section [6 we give a formulation of the vanishing theorem of 14, in a form suitable for this paper. Let $\mathfrak{a}_{\mathrm{q}}$ be a maximal abelian subspace of $\mathfrak{p} \cap \mathfrak{q}$, the intersection of the -1 eigenspaces in $\mathfrak{g}$ for $\theta$ and $\sigma$, respectively. Let $\mathcal{P}_{\sigma}$ denote the set of $\sigma \theta$-stable parabolic subgroups of $G$ containing $A_{\mathrm{q}}$ : $=\exp \mathfrak{a}_{\mathrm{q}}$. For each $Q \in \mathcal{P}_{\sigma}$ we introduce a space $\mathcal{E}_{Q}^{\text {hyp }}(\mathrm{X}: \tau)$ of families $\left\{f_{\nu}\right\}$ of spherical generalized eigenfunctions on $\mathrm{X}$, depending meromorphically on the parameter $\nu \in \mathfrak{a}_{Q \mathrm{qc}}^{*}$. Here $\mathfrak{a}_{Q \mathrm{q}}:=\mathfrak{a}_{Q} \cap \mathfrak{q}$. The vanishing theorem asserts that $f_{\nu}=0$ for all $\nu$, as soon as the coefficient of $e^{\nu-\rho_{Q}}$ in the asymptotic expansion along $Q$ vanishes, for all $\nu$ in a non-empty open subset of $\mathfrak{a}_{Q \mathrm{qC}}^{*}$.

In Section 8 we recall the inversion formula of [12]. Let $\Sigma$ be the root system of $\mathfrak{a}_{\mathrm{q}}$ in $\mathfrak{g}$ and let $W$ be the associated Weyl group. Let $\Delta$ be the system of 
simple roots associated with the minimal element $P_{0}$ from $\mathcal{P}_{\sigma}$. For each $F \subset \Delta$, let $P_{F}=M_{F} A_{F} N_{F}$ denote the associated standard parabolic subgroup in $\mathcal{P}_{\sigma}$. Then the inversion formula is of the form

$$
f=\sum_{F \subset \Delta} t\left(\mathfrak{a}_{F \mathrm{q}}^{+}\right) T_{F}^{t} f
$$

where

$$
T_{F}^{t} f(x)=t\left(\mathfrak{a}_{F \mathbf{q}}^{+}\right)|W| \int_{i \mathfrak{a}_{F \mathfrak{q}}+\varepsilon_{F}} \int_{\mathbf{X}} K_{F}^{t}(\nu: x: y) f(y) d y d \mu_{F}(\nu) .
$$

Here $t$ is a choice of $W$-invariant even residue weight on $\Sigma$ and $\varepsilon_{F}$ is an element of $\mathfrak{a}_{F \mathrm{q}}^{+}$, sufficiently close to zero (if $F=\Delta$, we may take $\varepsilon_{F}=0$ ). Moreover, $d \mu_{F}$ is a suitable choice of Lebesgue measure on $i \mathfrak{a}_{F \mathbf{q}}^{*}+\varepsilon_{F}$. The kernel functions $K_{F}^{t}(\nu: x: y) \in \operatorname{End}\left(V_{\tau}\right)$ are obtained from residual operators acting on a combination of normalized and partial Eisenstein integrals for $P_{0}$, see [12 for details. They are meromorphic in the variable $\nu \in \mathfrak{a}_{Q \mathrm{qC}}^{*}$ and smooth spherical and $\mathbb{D}(\mathrm{X})$-finite in both of the variables $x, y \in \mathrm{X}$. Essentially, the idea is that the kernel $K_{F}^{t}$ determines the projection onto the part of $L^{2}(\mathrm{X}: \tau)$ determined by the induction from the standard parabolic subgroup $P_{F}$.

To make the above formula valid for Schwartz functions it is necessary to establish it with $\varepsilon_{F}=0$, for every $F$. This can be achieved by using Cauchy's formula, once we have established the regularity at $i \mathfrak{a}_{F \mathrm{q}}^{*}$ of the kernel functions $K_{F}^{t}(\nu, x, y)$ in the variable $\nu$. In addition to this we need estimates that are tempered in the variables $x, y$ with uniformity in $\nu \in i \mathfrak{a}_{Q q}^{*}$. All this is taken care of by a long inductive argument, that ranges over the Sections 12-21 We shall describe the structure of the argument, which goes by induction on the $\sigma$-split rank of $G$, at a later stage in this introduction.

In Section 9 we recall the definition of the generalized Eisenstein integral $E_{F}^{\circ}(\nu)$, for $\nu \in \mathfrak{a}_{F q \mathrm{qc}}^{*}$. In [12, Def. 10.7, see also [14, Remark 16.12, this Eisenstein integral was defined by means of a linear combination of residual operators (a so called Laurent functional) applied to the Eisenstein integral $E^{\circ}\left(P_{0}: \lambda\right)$ with respect to the variable $\lambda$. As a family in the parameter $\nu$, the generalized Eisenstein integral belongs to $\mathcal{E}_{P_{F}}^{\text {hyp }}(\mathrm{X}: \tau)$. Hence, in view of the vanishing theorem, it can be characterized uniquely in terms of its asymptotic behavior along $P_{F}$; see Theorem 9.3

In Corollary 10.6 we show that the generalized Eisenstein integral is tempered for regular imaginary values of $\nu$. This fact can be derived from a limitation on the asymptotic exponents, see Theorem 10.5 caused by the support of the residual operators. Here Thm. 3.15 of [11] is crucial. In the next section, in Proposition [11.6 we establish uniformly moderate estimates for the generalized Eisenstein integrals. These estimates come from similar estimates for $E^{\circ}\left(P_{0}: \cdot\right)$, which survive the application of the residual operators.

In Section 12 we start with the preparation of the long inductive argument mentioned above. The reductive symmetric pair $(G, H)$ is said to be of residue type if the following two conditions are fulfilled, (a) $G$ has compact center modulo $H$ and (b) the operator $T_{\Delta}^{t}$ is the projection onto the discrete part $L_{d}^{2}(\mathrm{X}: \tau)$ 
of $L^{2}(\mathrm{X}: \tau)$ (which may be trivial). Condition (b) implies that the mentioned operator, and hence the associated 'discrete' kernel $K_{\Delta}^{t}$, is independent of the particular choice of residue weight $t$. Moreover, from condition (b) it follows that $L_{d}^{2}(\mathrm{X}: \tau)$ is finite dimensional, a result known for all pairs $(G, H)$ as a consequence of [0], see also Remark 12.7

We proceed with the induction in Section 13. A parabolic subgroup $P \in \mathcal{P}_{\sigma}$ is said to be of residue type if the pair $\left(M_{P}, M_{P} \cap H\right)$ is. A subset $F \subset \Delta$ is said to be of residue type, if the associated standard parabolic subgroup $P_{F}$ is. In the course of the inductive argument, many results in Sections 12-21 will initially be proved under the assumption that $(G, H)$ or a parabolic subgroup from $\mathcal{P}_{\sigma}$ is of residue type. Such results will always be indicated with the abbreviation (RT) following their declaration. The chain of results marked (RT) is needed in the induction step of Theorem 21.2 where by induction on the $\sigma$-split rank of $G$ it is shown that all groups from $\mathcal{P}_{\sigma}$ are of residue type. In particular, also all pairs $(G, H)$, with $G$ having compact center modulo $H$, are of residue type. It thus follows that the results marked (RT) are valid in the generality stated (see also Remark 12.2).

The kernel $K_{F}^{t}(\nu: \cdot: \cdot)$ is determined by its asymptotic expansion along $P_{F} \times P_{F}$, in view of the vanishing theorem. The coefficient of $e^{\nu-\rho_{F}} \otimes e^{-\nu-\rho_{F}}$ in this expansion is essentially the discrete kernel of $M_{F} / M_{F} \cap H$. If $F$ is of residue type, then the discrete kernel, and hence $K_{F}^{t}$, is independent of the particular choice of $t$. Therefore, so is the generalized Eisenstein integral. From then on we call this Eisenstein integral the normalized one and denote it by $E^{\circ}\left(P_{F}: \nu\right)$. It is a meromorphic function of $\nu \in \mathfrak{a}_{F \mathrm{qC}}^{*}$, with values in $C^{\infty}(\mathrm{X}) \otimes \operatorname{Hom}\left(\mathcal{A}_{2, F}, V_{\tau}\right)$, where $\mathcal{A}_{2, F}=L_{d}^{2}\left(\mathrm{X}_{F}: \tau_{F}\right)$. (Without the simplifying assumption mentioned above, the latter space is replaced by a suitable direct sum over ${ }^{F} \mathcal{W}$.) The unique characterization of the normalized Eisenstein integral by means of the vanishing theorem then allows us to define it for $P_{F}$ replaced by any parabolic subgroup $P \in \mathcal{P}_{\sigma}$ of residue type. In the case of the group, the characterization allows us to identify the normalized Eisenstein integral with Harish-Chandra's, as defined in [29, $\S 6$, Thm. 6; see Remark 13.9.

The definition of the normalized Eisenstein integral in turn allows us to define a kernel function $K_{P}$ for arbitrary $P \in \mathcal{P}_{\sigma}$ of residue type, generalizing the kernels for standard parabolic subgroups of residue type. In terms of the normalized Eisenstein integrals, the kernel is given by the formula

$$
K_{P}(\nu: x: y)=\left|W_{P}\right|^{-1} E^{\circ}(P: \nu: x) E^{*}(P: \nu: y) .
$$

Here $W_{P}$ is the subgroup of $W$ that corresponds to the Weyl group of $\mathrm{X}_{P}$, and $E^{*}(P: \nu: \cdot)$ is the dualized Eisenstein integral. The latter is the function in $C^{\infty}(\mathrm{X}) \otimes \operatorname{Hom}\left(V_{\tau}, \mathcal{A}_{2, P}\right)$ defined by

$$
E^{*}(P: \nu: y):=E^{\circ}(P:-\bar{\nu}: y)^{*} .
$$

Two parabolic subgroups $P, Q \in \mathcal{P}_{\sigma}$ are said to be associated, notation $P \sim Q$, if their $\sigma$-split components $\mathfrak{a}_{P q}$ and $\mathfrak{a}_{Q q}$ are conjugate under $W$. If $P, Q \in \mathcal{P}_{\sigma}$ are 
associated, and if one of them is of residue type, then so is the other. Moreover, the set

$$
W\left(\mathfrak{a}_{Q \mathrm{q}} \mid \mathfrak{a}_{P \mathrm{q}}\right)=\left\{\left.s\right|_{\mathfrak{a}_{P \mathrm{q}}} \mid s \in W, \quad s\left(\mathfrak{a}_{P \mathrm{q}}\right) \subset \mathfrak{a}_{Q \mathrm{q}}\right\}
$$

is non-empty. The main result of the section is Theorem 13.23 which asserts that the kernel function is unchanged if $P, \nu$ are replaced by $Q, s \nu$, with $Q \sim P$ and $s \in W\left(\mathfrak{a}_{Q \mathrm{q}} \mid \mathfrak{a}_{P \mathrm{q}}\right)$. For $P$ minimal, this result is a consequence of the Maass-Selberg relations for the Eisenstein integral $E^{\circ}(P: \cdot)$, in view of (1.2). For arbitrary $P$ of residue type the result follows from the minimal case by $W$-equivariance properties of the residue calculus.

In Section 14 we describe the action of $\mathbb{D}(\mathrm{X})$ on the normalized Eisenstein integral $E^{\circ}(P: \nu)$, for $P$ of residue type. The diagonalization of $\mathbb{D}\left(\mathrm{X}_{P}\right)$ on $L_{d}^{2}\left(\mathrm{X}_{P}: \tau_{P}\right)$, where $\tau_{P}:=\left.\tau\right|_{K \cap P}$, induces a simultaneous diagonalization of the action of $\mathbb{D}(\mathrm{X})$ on the Eisenstein integral, in view of the vanishing theorem, see Corollary 14.4. In the next section this result is used to show that the uniform moderate estimates of the Eisenstein integral can be improved to uniform tempered estimates, exploiting a technique that goes back to [5] and 42

In Section [16] we recall the mentioned result of [40] on the $\mathbb{D}(\mathrm{X})$-characters of the discrete series in Theorem 16.1. This result is of crucial importance for Section [17] where we determine the constant term of the normalized Eisenstein integral. In addition we use the theory of the constant term as developed in 17, see also 30.

The constant term of $E^{\circ}(P: \nu)$ along a $Q \in \mathcal{P}_{\sigma}$ with $Q \sim P$ describes $e^{\rho_{Q}}$ times the top order asymptotic behavior along $Q$; it is given by

$$
E_{Q}^{\circ}(P: \nu: m a) \psi=\sum_{s \in W\left(\mathfrak{a}_{Q \mathrm{q}} \mid \mathfrak{a}_{P \mathrm{q}}\right)} a^{s \nu}\left[C_{Q \mid P}^{\circ}(s: \nu) \psi\right](m),
$$

for $\nu \in \mathfrak{a}_{P \mathrm{q}}^{*}$ generic, $a \in A_{Q \mathrm{q}}$ and $m \in \mathrm{X}_{Q}$. Here $C_{Q \mid P}^{\circ}(s: \nu)$, the normalized $C$-function, is a meromorphic $\operatorname{Hom}\left(\mathcal{A}_{2, P}, \mathcal{A}_{2, Q}\right)$-valued function of $\nu \in \mathfrak{a}_{P \mathrm{qC}}^{*}$. For the description of the constant term without the simplifying assumption mentioned above, see Corollary 17.7

In Section 18 we derive the Maass-Selberg relations from the invariance property of the kernel (1.2) mentioned above. They assert the following identity of meromorphic functions in the variable $\nu \in \mathfrak{a}_{P q}^{*}$,

$$
C_{Q \mid P}^{\circ}(s:-\bar{\nu})^{*} C_{Q \mid P}^{\circ}(s: \nu)=I,
$$

for $P, Q \in \mathcal{P}_{\sigma}$ associated and of residue type, and for $s \in W\left(\mathfrak{a}_{Q \mathrm{q}} \mid \mathfrak{a}_{P \mathrm{q}}\right)$. In particular, it follows that the normalized $C$-functions are unitary for imaginary $\nu$. This in turn shows that the constant term (1.3) is regular for imaginary $\nu$. By a result from [7] this implies that the Eisenstein integral $E^{\circ}(P: \nu)$ is regular for imaginary $\nu$, see Theorem 18.8 Because of the uniform tempered estimates formulated in Corollary 18.12 it becomes possible to define a spherical Fourier transform $\mathcal{F}_{P}$ in the next section by the formula

$$
\mathcal{F}_{P} f(\nu)=\int_{\mathrm{X}} E^{*}(P: \nu: x) f(x) d x,
$$


for $f \in \mathcal{C}(\mathrm{X}: \tau)$, the space of $\tau$-spherical Schwartz functions on X. Proposition [19.6 asserts that $\mathcal{F}_{P}$ is a continuous linear map into the Euclidean Schwartz space $\mathcal{S}\left(i \mathfrak{a}_{P \mathrm{q}}^{*}\right) \otimes \mathcal{A}_{2, P}$, if $P$ is of residue type. In Section [20] it is shown, using a result from [7], that the adjoint wave packet transform, given by the formula

$$
\mathcal{J}_{P} \varphi(x)=\int_{i \mathfrak{a}_{P \mathrm{q}}^{*}} E^{\circ}(P: \nu: x) \varphi(\nu) d \mu_{P}(\nu)
$$

is a continuous linear map from $\mathcal{S}\left(i \mathfrak{a}_{P q}^{*}\right) \otimes \mathcal{A}_{2, P}$ into $\mathcal{C}(\mathrm{X}: \tau)$, see Theorem 20.3. Here $d \mu_{P}$ is Lebesgue measure on $i \mathfrak{a}_{P \mathrm{q}}^{*}$, normalized as in Section [5]

In Section 21] the long inductive argument is completed as follows. In the proof of Theorem 21.2 it is shown that every $P \in \mathcal{P}_{\sigma}$ is of residue type, by induction on the $\sigma$-split rank of $G$. The hypothesis of the induction step implies that one may assume that $G$ has compact center modulo $H$ and that every $F \subsetneq \Delta$ is of residue type. In view of the regularity of the normalized Eisenstein integrals, hence of the kernels $K_{F}(\nu: \cdot: \cdot)$, for $F \subsetneq \Delta$ and $\nu \in i \mathfrak{a}_{F \mathrm{q}}^{*}$, the formula (1.1) becomes valid with $\varepsilon_{F}=0$ for every subset $F \subset \Delta$ (recall that $\varepsilon_{\Delta}=0$ ). Moreover, by the definition of the transforms $\mathcal{F}_{F}:=\mathcal{F}_{P_{F}}$ and $\mathcal{J}_{F}:=\mathcal{J}_{P_{F}}$, it takes the form

$$
f=T_{\Delta}^{t} f+\sum_{F \subsetneq \Delta}\left[W: W_{F}\right] t\left(P_{F}\right) \mathcal{J}_{F} \mathcal{F}_{F} f .
$$

From this one reads off that $T_{\Delta}^{t}$ maps $C_{c}^{\infty}(\mathrm{X}: \tau)$ into $\mathcal{C}(\mathrm{X}: \tau)$, from which it readily follows that $T_{\Delta}^{t}$ is the restriction to $C_{c}^{\infty}(\mathrm{X}: \tau)$ of the orthogonal projection onto $L_{d}^{2}(\mathrm{X}: \tau)$. This argument completes the induction step; moreover, at the same time it shows that

$$
I=\sum_{F \subset \Delta}\left[W: W_{F}\right] t\left(P_{F}\right) \mathcal{J}_{F} \mathcal{F}_{F}
$$

on $C_{c}^{\infty}(\mathrm{X}: \tau)$ and hence on $\mathcal{C}(\mathrm{X}: \tau)$ by continuity and density. It is at this point that the role of the residue weight in the harmonic analysis becomes clear. Define the equivalence relation $\sim$ on the powerset $2^{\Delta}$ by $F \sim F^{\prime} \Longleftrightarrow P_{F} \sim P_{F^{\prime}}$. Then by the Maass-Selberg relations the composed transform $\mathcal{J}_{F} \mathcal{F}_{F}$ only depends on the class of $F$ in $2^{\Delta} / \sim$. Collecting the terms in (1.6) according to such classes we obtain, by an easy counting argument, the following Fourier inversion formula which is independent of the choice of residue weight

$$
I=\sum_{[F] \in 2^{\Delta} / \sim}\left[W: W_{F}^{*}\right] \mathcal{J}_{F} \mathcal{F}_{F}
$$

with $W_{F}^{*}$ the normalizer of $\mathfrak{a}_{F \mathrm{q}}$ in $W$. In other words, the residue weight describes the weight by which an element in the class of $F$ contributes to the above inversion formula.

In Section 22 we give a precise description of the kernels and images of the Fourier transforms and their adjoints. This leads to the spherical Plancherel theorem for Schwartz functions, Theorem 23.1 and the similar theorem for $L^{2}$-functions, Theorem 23.4 In particular, the summands in (1.7) extend to 
$L^{2}(\mathrm{X}: \tau)$ and become orthogonal projections onto mutually orthogonal subspaces.

We are grateful to the organizers of the special year at the Mittag-Leffler Institute, during which these results were found, and we thank Mogens FlenstedJensen for several helpful discussions. We are also grateful to Jacques Carmona and Patrick Delorme for informing us about their results on the Maass-Selberg relations, which played a crucial role for us, as mentioned above.

\section{Notation and preliminaries}

Throughout this paper, $G$ will be a real reductive group of Harish-Chandra's class, $\sigma$ an involution of $G$ and $H$ an open subgroup of $G^{\sigma}$, the set of fixed points for $\sigma$. The associated reductive symmetric space is denoted by

$$
\mathrm{X}=G / H .
$$

The algebra of $G$-invariant differential operators on $\mathrm{X}$ is denoted by $\mathbb{D}(\mathrm{X})$.

We fix a Cartan involution $\theta$ of $G$ that commutes with $\sigma$; thus, the associated maximal compact subgroup $K=G^{\theta}$ is $\sigma$-invariant. We follow the convention to denote Lie groups with roman capitals, and their Lie algebras with the corresponding gothic lower cases. In particular, $\mathfrak{g}$ denotes the Lie algebra of $G$. The infinitesimal involutions of $\mathfrak{g}$ associated with $\theta$ and $\sigma$ are denoted by the same symbols.

We equip $\mathfrak{g}$ with a $G$-invariant non-degenerate bilinear form $B$ that restricts to the Killing form on $[\mathfrak{g}, \mathfrak{g}]$, that is positive definite on $\mathfrak{p}$, negative definite on $\mathfrak{k}$, and for which $\sigma$ is symmetric. Then $\langle X, Y\rangle:=-B(X, \theta Y)$ defines a positive definite inner product on $\mathfrak{g}$ for which the involutions $\theta$ and $\sigma$ are symmetric. Accordingly, the decompositions $\mathfrak{g}=\mathfrak{k} \oplus \mathfrak{p}=\mathfrak{h} \oplus \mathfrak{q}$ into the +1 and -1 eigenspaces of these involutions, respectively, are orthogonal. If $\mathfrak{v} \subset \mathfrak{g}$ is a linear subspace, we agree to identify $\mathfrak{v}^{*}$ with a linear subspace of $\mathfrak{g}$ via the inner product $\langle\cdot, \cdot\rangle$. Finally, we equip the linear dual $\mathfrak{g}^{*}$ of $\mathfrak{g}$ with the dual inner product, and the complexified dual $\mathfrak{g}_{\mathbb{C}}^{*}$ with its complex bilinear extension.

We fix a maximal abelian subspace $\mathfrak{a}_{\mathrm{q}}$ of $\mathfrak{p} \cap \mathfrak{q}$ and denote the associated system of restricted roots by $\Sigma=\Sigma\left(\mathfrak{g}, \mathfrak{a}_{\mathrm{q}}\right)$. This is a, possibly non-reduced, root system; the associated Weyl group is denoted by $W=W(\Sigma)$. We recall that $W \simeq N_{K}\left(\mathfrak{a}_{\mathrm{q}}\right) / Z_{K}\left(\mathfrak{a}_{\mathrm{q}}\right)$, naturally. Accordingly, the natural image of $N_{K \cap H}\left(\mathfrak{a}_{\mathrm{q}}\right)$ in $W$ is denoted by $W_{K \cap H}$.

If $P$ is a parabolic subgroup of $G$, we denote its Langlands decomposition by $P=M_{P} A_{P} N_{P}$ and put $M_{1 P}:=M_{P} A_{P}$. A parabolic subgroup that is invariant under the composed involution $\sigma \theta$ is called a $\sigma$-parabolic subgroup. The set of $\sigma$-parabolic subgroups containing $A_{\mathrm{q}}:=\exp \mathfrak{a}_{\mathrm{q}}$ is finite and denoted by $\mathcal{P}_{\sigma}$.

We shall briefly recall the structure of the parabolic subgroups from $\mathcal{P}_{\sigma}$, meanwhile fixing notation. For details we refer to [14], $\S 2$. If $P \in \mathcal{P}_{\sigma}$, then $M_{P}$ and $A_{P}$ are $\sigma$-invariant, and $\sigma N_{P}=\theta N_{P}=\bar{N}_{P}$. The algebra $\mathfrak{a}_{P}$ is $\sigma$-invariant, hence decomposes as $\mathfrak{a}_{P}=\mathfrak{a}_{P \mathrm{~h}} \oplus \mathfrak{a}_{P \mathrm{q}}$, the vector sum of the intersections of $\mathfrak{a}_{P}$ 
with $\mathfrak{h}$ and $\mathfrak{q}$, respectively. We put $A_{P \mathfrak{q}}:=\exp \mathfrak{a}_{P \mathfrak{q}}$ and $M_{P \sigma}:=M_{P}\left(A_{P} \cap H\right)$ and call $P=M_{P \sigma} A_{P \mathrm{q}} N_{P}$ the $\sigma$-Langlands decomposition of $P$.

As usual, we denote by $\rho_{P}$ the linear functional $\frac{1}{2} \operatorname{tr}\left[\left.\operatorname{ad}(\cdot)\right|_{\mathfrak{n}_{P}}\right] \in \mathfrak{a}_{P}^{*}$. The following lemma is of importance in the theory of induced representations.

Lemma 2.1 Let $P \in \mathcal{P}_{\sigma}$. Then $\rho_{P}$ belongs to $\mathfrak{a}_{P \mathrm{q}}^{*}$.

Proof: The algebras $\mathfrak{a}_{P}$ and $\mathfrak{n}_{P}$ are $\sigma \theta$-invariant, hence $-\sigma \rho_{P}=\sigma \theta \rho_{P}=\rho_{P}$. This implies that $\rho_{P}$ vanishes on $\mathfrak{a}_{P \mathrm{~h}}$, hence belongs to $\mathfrak{a}_{P \mathrm{q}}^{*}$.

The space $\mathfrak{a}_{P \mathrm{q}}$ is contained in $\mathfrak{a}_{\mathrm{q}}$. Let $\Sigma_{P}$ denote the collection of roots from $\Sigma$ that vanish on $\mathfrak{a}_{P \mathrm{q}}$. Then

$$
\mathfrak{a}_{P \mathrm{q}}=\cap_{\alpha \in \Sigma_{P}} \operatorname{ker} \alpha .
$$

The subgroup of $W$ generated by the reflections in the roots of $\Sigma_{P}$ is denoted by $W_{P}$. It equals the centralizer of $\mathfrak{a}_{P \mathrm{q}}$ in $W$.

Let $\Sigma(P)$ be the collection of roots from $\Sigma$ that occur in $\mathfrak{n}_{P}$ as an $\mathfrak{a}_{\mathrm{q}}$-weight. Then $\mathfrak{n}_{P}$ is the vectorial direct sum of the root spaces $\mathfrak{g}_{\alpha}$, for $\alpha \in \Sigma(P)$. We put

$$
\Sigma_{r}(P):=\Sigma\left(\mathfrak{n}_{P}, \mathfrak{a}_{P \mathrm{q}}\right)=\left\{\left.\alpha\right|_{\mathfrak{a}_{P \mathrm{q}}} \mid \alpha \in \Sigma(P)\right\} .
$$

The set

$$
\mathfrak{a}_{P \mathrm{q}}^{+}:=\left\{X \in \mathfrak{a}_{P \mathrm{q}} \mid \alpha(X)>0 \quad \text { for all } \quad \alpha \in \Sigma(P)\right\}
$$

is non-empty. Therefore, the elements of $\Sigma_{r}(P)$ are non-zero linear functionals on $\mathfrak{a}_{P \mathrm{q}}$. Moreover, $\mathfrak{a}_{P \mathrm{q}}^{+}$is a connected component of the complement $\mathfrak{a}_{P \mathrm{q}}^{\text {reg }}$ of the union of their null spaces. We put $A_{P \mathrm{q}}^{+}:=\exp \mathfrak{a}_{P \mathrm{q}}^{+}$.

The collection of weights in $\Sigma_{r}(P)$ that cannot be expressed as a sum of two elements of $\Sigma_{r}(P)$ is denoted by $\Delta_{r}(P)$. We recall from [14, beginning of $\S 3$, that the set $\Delta_{r}(P)$ is linearly independent over $\mathbb{R}$ and spans $\Sigma_{r}(P)$ over $\mathbb{N}$.

If $X \in \mathfrak{a}_{\mathrm{q}}$, then $X \in \mathfrak{p}$, hence ad $X$ diagonalizes with real eigenvalues. It is well known that the sum of the eigenspaces for the non-negative eigenvalues is a parabolic subalgebra of $\mathfrak{g}$. Its $\theta$-stable Levi component $\mathfrak{m}_{1 X}$ and its nilpotent radical $\mathfrak{n}_{X}$ are given by

$$
\mathfrak{m}_{1 X}=\operatorname{ker} \operatorname{ad} X, \quad \mathfrak{n}_{X}=\oplus_{\alpha \in \Sigma, \alpha(X)>0} \quad \mathfrak{g}_{\alpha} .
$$

The associated parabolic subgroup of $G$ is denoted by $P_{X}$. If $P \in \mathcal{P}_{\sigma}$ and $X \in \mathfrak{a}_{P q}^{+}$, then it follows from [14, Eqn. (2.4), that $P=P_{X}$. From $\sigma \theta X=X$ it follows that $P_{X} \in \mathcal{P}_{\sigma}$.

Let $\sim$ be the relation of parabolic equivalence on $\mathfrak{a}_{\mathrm{q}}$, with respect to the root system $\Sigma$. Thus, $X \sim Y$ if and only if for each $\alpha \in \Sigma$ we have $\alpha(X)>$ $0 \Longleftrightarrow \alpha(Y)>0$. It readily follows from the definition given above that $X \sim Y \Longleftrightarrow P_{X}=P_{Y}$.

Lemma 2.2 The map $P \mapsto \mathfrak{a}_{P \mathrm{q}}^{+}$is a bijection from $\mathcal{P}_{\sigma}$ onto the set $\mathfrak{a}_{\mathrm{q}} / \sim$ of parabolic equivalence classes. 
Proof: If $P \in \mathcal{P}_{\sigma}$ and $X \in \mathfrak{a}_{P \mathrm{q}}^{+}$then $P=P_{X}$, as said above. Hence, the map $X \mapsto P_{X}$ is a surjection from $\mathfrak{a}_{\mathrm{q}}$ onto $\mathcal{P}_{\sigma}$. By the last assertion before Lemma 2.2 the map factors to a bijection from $\mathfrak{a}_{\mathrm{q}} / \sim$ onto $\mathcal{P}_{\sigma}$. If $X \in \mathfrak{a}_{\mathrm{q}}$, let $P=P_{X}$. Then $\Sigma(P)=\{\alpha \in \Sigma \mid \alpha(X)>0\}$, hence $\Sigma_{P}=\{\alpha \in \Sigma \mid \alpha(X)=0\}$, and we see that $[X]=\mathfrak{a}_{P \mathrm{q}}^{+}$. Thus, $P \mapsto \mathfrak{a}_{P \mathrm{q}}^{+}$is the inverse to $[X] \mapsto P_{X}$.

It follows from the description in Lemma 2.2 that the Weyl group $W$ acts on the finite set $\mathcal{P}_{\sigma}$. We recall from [11], Def. 3.2, that a residue weight on $\Sigma$ is a map $\mathfrak{a}_{\mathrm{q}}^{*} / \sim \rightarrow[0,1]$ such that for every $Q \in \mathcal{P}_{\sigma}$,

$$
\sum_{P \in \mathcal{P}_{\sigma}, \mathfrak{a}_{P \mathrm{q}}=\mathfrak{a}_{Q \mathrm{q}}} t\left(\mathfrak{a}_{P \mathrm{q}}^{+}\right)=1 .
$$

The collection of residue weights on $\Sigma$ is denoted by $\mathrm{WT}(\Sigma)$. Via the bijection of Lemma 2.2 a weight $t \in \mathrm{WT}(\Sigma)$ will also be viewed as a map $t: \mathcal{P}_{\sigma} \rightarrow[0,1]$. A residue weight $t \in \mathrm{WT}(\Sigma)$ is said to be $W$-invariant if $t\left(w \mathfrak{a}_{P \mathrm{q}}^{+}\right)=t\left(\mathfrak{a}_{P \mathrm{q}}^{+}\right)$for all $P \in \mathcal{P}_{\sigma}$ and $w \in W$, and even if $t\left(\mathfrak{a}_{P \mathfrak{q}}^{+}\right)=t\left(-\mathfrak{a}_{P \mathrm{q}}^{+}\right)$for all $P \in \mathcal{P}_{\sigma}$.

Let $\mathcal{P}_{\sigma}^{\min }$ the collection of minimal elements in $\mathcal{P}_{\sigma}$. Then $P \mapsto \mathfrak{a}_{P \mathrm{q}}^{+}$is a bijection from $\mathcal{P}_{\sigma}^{\min }$ onto the collection of open chambers for $\Sigma$ in $\mathfrak{a}_{\mathrm{q}}$. To emphasize this, we shall also write $\mathfrak{a}_{\mathrm{q}}^{+}(P):=\mathfrak{a}_{P \mathrm{q}}^{+}$and $A_{\mathrm{q}}^{+}(P):=A_{P \mathrm{q}}^{+}$for $P \in \mathcal{P}_{\sigma}^{\min }$. Accordingly, $W$ acts simply transitively on $\mathcal{P}_{\sigma}^{\min }$. Note that for $P \in \mathcal{P}_{\sigma}^{\min }$, $\Sigma(P)=\Sigma_{r}(P)$ is a positive system for $\Sigma$ and $\Delta(P):=\Delta_{r}(P)$ the associated collection of simple roots.

We fix a system $\Sigma^{+}$of positive roots for $\Sigma$; let $\Delta$ be the associated collection of simple roots. Given $F \subset \Delta$ we define

$$
\mathfrak{a}_{F \mathrm{q}}:=\cap_{\alpha \in F} \operatorname{ker} \alpha
$$

and denote by $\mathfrak{a}_{F \mathrm{q}}^{+}$the subset of elements $X \in \mathfrak{a}_{F \mathrm{q}}$ such that $\beta(X)>0$ for $\beta \in \Delta \backslash F$. Then $\mathfrak{a}_{F \mathrm{q}}^{+}$is a parabolic equivalence class. The associated parabolic subgroup $P_{F}$ is called the standard parabolic subgroup determined by $F$. We adopt the convention to replace an index or superscript $P_{F}$ by $F$. In particular, the Langlands decomposition of $P_{F}$ is denoted by $P_{F}=M_{F} A_{F} N_{F}$ and the centralizer of $\mathfrak{a}_{F \mathrm{q}}$ in $W$ by $W_{F}$. Let

$$
W^{F}:=\left\{s \in W \mid s(F) \subset \Sigma^{+}\right\} .
$$

Then the canonical map $W \rightarrow W / W_{F}$ induces a bijection $W^{F} \rightarrow W / W_{F}$.

We write $P_{0}$ for $P_{\emptyset}, P_{0}=M A N_{0}$ for its Langlands decomposition and $M_{1}:=$ $M A$. Then $P=M_{1} N_{P}$ for every $P \in \mathcal{P}_{\sigma}^{\min }$.

If $P \in \mathcal{P}_{\sigma}$ and $v \in N_{K}\left(\mathfrak{a}_{\mathrm{q}}\right)$, we define

$$
\mathrm{X}_{P, v}:=M_{P} / M_{P} \cap v H v^{-1} .
$$

Here $M_{P}$ is a real reductive group of Harish-Chandra's class and $M_{P} \cap v H v^{-1}$ is an open subgroup of the group of fixed points for the involution $\sigma^{v}: M_{P} \rightarrow M_{P}$ defined by $\sigma^{v}(m)=v \sigma\left(v^{-1} m v\right) v^{-1}$. Thus, the space in (2.2) is a reductive 
symmetric space in the class under consideration. Moreover, $\left.\theta\right|_{M_{P}}$ is a Cartan involution of $M_{P}$ that commutes with $\sigma^{v}$; the associated maximal compact subgroup is $K_{P}:=K \cap M_{P}$.

Note that as (an isomorphism class of) a $M_{P}$-homogeneous space, the symmetric space $\mathrm{X}_{P, v}$ depends on $v$ through its class in the double coset space $W_{P} \backslash W / W_{K \cap H}$. Throughout this paper, ${ }^{P} \mathcal{W}$ will denote a choice of representatives in $N_{K}\left(\mathfrak{a}_{\mathrm{q}}\right)$ of $W_{P} \backslash W / W_{K \cap H}$. In general, if $f$ is a surjective map from a set $A$ onto a set $B$, then by a choice of representatives for $B$ in $A$, we mean a subset $\mathcal{B} \subset A$ such that $\left.f\right|_{\mathcal{B}}: \mathcal{B} \rightarrow B$ is a bijection.

Let $* \mathfrak{a}_{P \mathrm{q}}$ denote the orthocomplement of $\mathfrak{a}_{P \mathrm{q}}$ in $\mathfrak{a}_{\mathrm{q}}$. Then

$$
{ }^{*} \mathfrak{a}_{P \mathrm{q}}=\mathfrak{m}_{P} \cap \mathfrak{a}_{\mathrm{q}} .
$$

Moreover, for every $v \in N_{K}\left(\mathfrak{a}_{\mathrm{q}}\right)$, this space is the analogue of $\mathfrak{a}_{\mathrm{q}}$ for the triple $\left(M_{P}, K_{P}, M_{P} \cap v H v^{-1}\right)$, see [14, text following (3.4).

In analogy with (2.2), we define $\mathrm{X}_{1 P, v}:=M_{1 P} / M_{1 P} \cap v H v^{-1}$, for $P \in \mathcal{P}_{\sigma}$ and $v \in N_{K}\left(\mathfrak{a}_{\mathrm{q}}\right)$. The multiplication map $M_{P} \times A_{P \mathrm{q}} \rightarrow M_{1 P}$ induces a diffeomorphism

$$
\mathrm{X}_{1 P, v} \simeq \mathrm{X}_{P, v} \times A_{P q} .
$$

If $v=e$, we agree to omit $v$ in the notation of the spaces in this product, so that $\mathrm{X}_{1 P}=M_{1 P} / M_{1 P} \cap H \simeq M_{P} / M_{P} \cap H \times A_{P q}$.

We end this section with collecting some basic facts about $\theta$-stable Cartan subspaces of $\mathfrak{q}$, meanwhile fixing notation. We define the dual real form $\mathfrak{g}^{d}$ of $\mathfrak{g}$ as the real form of $\mathfrak{g}_{\mathbb{C}}$ given by $\mathfrak{g}^{d}=\operatorname{ker}(\sigma \theta-I) \oplus i \operatorname{ker}(\sigma \theta+I)$. Let $\theta_{\mathbb{C}}$ and $\sigma_{\mathbb{C}}$ be the complex linear extensions of $\theta$ and $\sigma$, respectively. Then $\theta^{d}:=\left.\sigma_{\mathbb{C}}\right|_{\mathfrak{g}^{d}}$ is a Cartan involution of $\mathfrak{g}^{d}$ and $\sigma^{d}:=\left.\theta_{\mathbb{C}}\right|_{\mathfrak{g}^{d}}$ is an involution of $\mathfrak{g}^{d}$ commuting with $\theta^{d}$.

If $\mathfrak{v}$ is any $\sigma$ - and $\theta$-stable subspace of $\mathfrak{g}$, then ${ }^{\mathrm{d}} \mathfrak{v}:=\mathfrak{v}_{\mathbb{C}} \cap \mathfrak{g}^{\mathrm{d}}$ is a $\sigma^{\mathrm{d}}$ - and $\theta^{\mathrm{d}}$-stable subspace of $\mathfrak{g}^{\mathrm{d}}$, whose complexification equals that of $\mathfrak{v}$.

If $\mathfrak{b}$ is a $\theta$-stable Cartan subspace of $\mathfrak{q}$, then $\mathfrak{b}=\mathfrak{b}_{\mathrm{k}} \oplus \mathfrak{b}_{\mathrm{p}}$, where $\mathfrak{b}_{\mathrm{k}}:=\mathfrak{b} \cap \mathfrak{k}$ and $\mathfrak{b}_{\mathrm{p}}:=\mathfrak{b} \cap \mathfrak{p}$. Moreover,

$$
{ }^{\mathrm{d}} \mathfrak{b}:=i \mathfrak{b}_{\mathrm{k}} \oplus \mathfrak{b}_{\mathrm{p}}
$$

is a $\sigma^{\mathrm{d}}$-stable maximal abelian subspace of $\mathfrak{p}^{\mathrm{d}}$. We denote by $\Sigma(\mathfrak{b})$ the root system of ${ }^{\mathrm{d}} \mathfrak{b}$ in $\mathfrak{g}^{\mathrm{d}}$, by $W(\mathfrak{b})$ the associated Weyl group and by $I(\mathfrak{b})$ the space of $W(\mathfrak{b})$-invariants in $S(\mathfrak{b})$, the symmetric algebra of $\mathfrak{b}_{\mathbb{C}}$. Moreover, we denote the associated Harish-Chandra isomorphism by

$$
\gamma_{\mathrm{d} \mathfrak{b}}: \quad U\left(\mathfrak{g}^{\mathrm{d}}\right)^{\mathfrak{k}^{\mathrm{d}}} / U\left(\mathfrak{g}^{\mathrm{d}}\right)^{\mathfrak{k}^{\mathrm{d}}} \cap U\left(\mathfrak{g}^{\mathrm{d}}\right) \mathfrak{k}^{\mathrm{d}} \rightarrow I(\mathfrak{b}) .
$$

As usual, if $\mathfrak{l}$ is a real Lie algebra, we denote by $U(\mathfrak{l})$ the universal algebra of its complexification. Via the natural isomorphism

$$
\mathbb{D}(\mathrm{X}) \simeq U(\mathfrak{g})^{\mathfrak{h}} / U(\mathfrak{g})^{\mathfrak{h}} \cap U(\mathfrak{g}) \mathfrak{h}=U\left(\mathfrak{g}^{\mathrm{d}}\right)^{\mathfrak{k}^{\mathrm{d}}} / U\left(\mathfrak{g}^{\mathrm{d}}\right)^{\mathfrak{k}^{\mathrm{d}}} \cap U\left(\mathfrak{g}^{\mathrm{d}}\right) \mathfrak{k}^{\mathrm{d}},
$$

see [5], Lemma 2.1, we shall identify the algebra $\mathbb{D}(\mathrm{X})$ with the algebra on the left-hand side of (2.4) and thus view the Harish-Chandra isomorphism $\gamma_{\mathrm{d} \mathfrak{b}}$ as an algebra isomorphism from $\mathbb{D}(\mathrm{X})$ onto $I(\mathfrak{b})$; as such it is denoted by $\gamma=\gamma_{\mathfrak{b}}$. 
If $P \in \mathcal{P}_{\sigma}$ and $\mathfrak{b}$ a $\theta$-stable Cartan subspace of $\mathfrak{q}$ containing $\mathfrak{a}_{P \mathfrak{q}}$, we agree to write ${ }^{*} \mathfrak{b}_{P}:=\mathfrak{b} \cap \mathfrak{m}_{P}$. Then ${ }^{*} \mathfrak{b}_{P}$ is a $\theta$-stable Cartan subspace of $\mathfrak{m}_{P} \cap \mathfrak{q}$ and

$$
\mathfrak{b}={ }^{*} \mathfrak{b}_{P} \oplus \mathfrak{a}_{P \mathfrak{q}},
$$

with orthogonal summands. If $P$ is minimal, then $\mathfrak{b}$ is maximally split, and we suppress the index $P$, so that $\mathfrak{b}={ }^{*} \mathfrak{b} \oplus \mathfrak{a}_{\mathrm{q}}$. We shall write $W\left({ }^{*} \mathfrak{b}_{P}\right)$ for the Weyl group of the pair $\left(\mathfrak{m}_{P \mathbb{C}},{ }^{*} \mathfrak{b}_{P}\right)$. Via the decomposition (2.6) this Weyl group is naturally identified with $W_{P}(\mathfrak{b})$, the centralizer of $\mathfrak{a}_{P \mathrm{q}}$ in $W(\mathfrak{b})$.

\section{$3 \quad$ Weyl groups}

In this section we discuss a straightforward generalization of well known results on Weyl groups, see [30, p. 111.

If $\mathfrak{a}_{1}$ and $\mathfrak{a}_{2}$ are abelian subspaces of $\mathfrak{p}$, then following [30, p. 112, we define the set

$$
W\left(\mathfrak{a}_{2} \mid \mathfrak{a}_{1}\right):=\left\{s \in \operatorname{Hom}\left(\mathfrak{a}_{1}, \mathfrak{a}_{2}\right)|\exists g \in G: \quad s=\operatorname{Ad}(g)|_{\mathfrak{a}_{1}}\right\} .
$$

From the definition it is obvious that the set $W\left(\mathfrak{a}_{2} \mid \mathfrak{a}_{1}\right)$ consists of injective linear maps. In particular, if $\operatorname{dim} \mathfrak{a}_{1}=\operatorname{dim} \mathfrak{a}_{2}$, it consists of linear isomorphisms. Finally, if $\mathfrak{a}_{1}=\mathfrak{a}_{2}$, the set is a subgroup of $\operatorname{GL}\left(\mathfrak{a}_{1}\right)=\operatorname{GL}\left(\mathfrak{a}_{2}\right)$. We note that $W\left(\mathfrak{a}_{1} \mid \mathfrak{a}_{1}\right)$ naturally acts from the right on $W\left(\mathfrak{a}_{2} \mid \mathfrak{a}_{1}\right)$, whereas $W\left(\mathfrak{a}_{2} \mid \mathfrak{a}_{2}\right)$ naturally acts from the left. If $\operatorname{dim} \mathfrak{a}_{1}=\operatorname{dim} \mathfrak{a}_{2}$, then both of these actions are transitive and free. If $\mathfrak{a}_{\mathfrak{p}}$ is a maximal abelian subspace of $\mathfrak{p}$, then by $W\left(\mathfrak{g}, \mathfrak{a}_{\mathfrak{p}}\right)$ we denote the Weyl group of $\Sigma\left(\mathfrak{g}, \mathfrak{a}_{\mathfrak{p}}\right)$, the root system of $\mathfrak{a}_{\mathfrak{p}}$ in $\mathfrak{g}$.

Lemma 3.1 Let $\mathfrak{a}_{1}$ and $\mathfrak{a}_{2}$ be abelian subspaces of $\mathfrak{p}$.

(a) The set $W\left(\mathfrak{a}_{1} \mid \mathfrak{a}_{2}\right)$ is finite.

(b) If $\varphi \in \operatorname{Int}\left(\mathfrak{g}_{\mathrm{C}}\right)$ maps $\mathfrak{a}_{1}$ into $\mathfrak{a}_{2}$ then $\left.\varphi\right|_{\mathfrak{a}_{1}} \in W\left(\mathfrak{a}_{2} \mid \mathfrak{a}_{1}\right)$.

(c) If $s \in W\left(\mathfrak{a}_{2} \mid \mathfrak{a}_{1}\right)$, then there exists a $k \in K_{e}$ such that $s=\left.\operatorname{Ad}(k)\right|_{\mathfrak{a}_{1}}$.

(d) Assume that $\mathfrak{a}_{1}$ and $\mathfrak{a}_{2}$ are contained in a maximal abelian subspace $\mathfrak{a}_{\mathfrak{p}}$ of p. Then

$$
W\left(\mathfrak{a}_{2} \mid \mathfrak{a}_{1}\right)=\left\{t \in \operatorname{Hom}\left(\mathfrak{a}_{1}, \mathfrak{a}_{2}\right)\left|\exists s \in W\left(\mathfrak{g}, \mathfrak{a}_{\mathfrak{p}}\right): t=s\right|_{\mathfrak{a}_{1}}\right\} .
$$

Proof: All assertions are immediate consequences of Corollaries 1, 2 and 3 of [30], p. 112.

\section{Corollary 3.2}

(a) $W=W\left(\mathfrak{a}_{\mathrm{q}} \mid \mathfrak{a}_{\mathrm{q}}\right)$.

(b) Let $\mathfrak{a}_{\mathfrak{p}}$ be a maximal abelian subspace of $\mathfrak{p}$, containing $\mathfrak{a}_{\mathrm{q}}$. Then the map $\left.k \mapsto \operatorname{Ad}(k)\right|_{\mathfrak{a}_{\mathfrak{q}}}$ is a surjection from $N_{K_{e}}\left(\mathfrak{a}_{\mathrm{q}}\right) \cap N_{K_{e}}\left(\mathfrak{a}_{\mathfrak{p}}\right)$ onto $W$. 
Proof: The map $\left.k \mapsto \operatorname{Ad}(k)\right|_{\mathfrak{a}_{\mathrm{q}}}$ induces a natural isomorphism $N_{K}\left(\mathfrak{a}_{\mathrm{q}}\right) / Z_{K}\left(\mathfrak{a}_{\mathrm{q}}\right) \simeq$ $W$, see, e.g., 4], Lemma 1.2. Hence, $W \subset W\left(\mathfrak{a}_{\mathrm{q}} \mid \mathfrak{a}_{\mathrm{q}}\right)$. For the converse inclusion, select a maximal abelian subspace $\mathfrak{a}_{\mathfrak{p}}$ of $\mathfrak{p}$, containing $\mathfrak{a}_{\mathrm{q}}$. Then by Lemma 3.1 (d), any element $t \in W\left(\mathfrak{a}_{\mathrm{q}} \mid \mathfrak{a}_{\mathrm{q}}\right)$ is the restriction of an element $s \in W\left(\mathfrak{g}, \mathfrak{a}_{\mathfrak{p}}\right)$. There exists a $k \in N_{K_{e}}\left(\mathfrak{a}_{\mathfrak{p}}\right)$ such that $s=\left.\operatorname{Ad}(k)\right|_{\mathfrak{a}_{\mathfrak{p}}}$. The element $k$ necessarily normalizes $\mathfrak{a}_{\mathrm{q}}$. Thus, we obtain the converse inclusion and also the validity of assertion (b).

The following lemma generalizes Lemma 1 of [30], p. 111. Let $\mathfrak{a}_{\mathrm{q}}$ be a maximal abelian subspace of $\mathfrak{p} \cap \mathfrak{q}$. Let $\mathfrak{a}_{\mathfrak{p}}$ be a maximal abelian subspace of $\mathfrak{p}$ containing $\mathfrak{a}_{\mathrm{q}}$, and $\mathfrak{j}$ a Cartan subalgebra of $\mathfrak{g}$ containing $\mathfrak{a}_{\mathfrak{p}}$. We denote by $W\left(\mathfrak{g}_{\mathbb{C}}, \mathfrak{j}_{\mathbb{C}}\right)$ the Weyl group of the root system of $\mathfrak{j}_{\mathbb{C}}$ in $\mathfrak{g}_{\mathrm{c}}$.

Lemma 3.3 Two elements of $\mathfrak{a}_{\mathrm{q}}$ are conjugate under $\operatorname{Int}\left(\mathfrak{g}_{\mathbb{C}}\right)$ if and only if they are conjugate under any one of the following groups

$$
W\left(\mathfrak{g}_{\mathbb{C}}, \dot{\mathfrak{j}}_{\mathbb{C}}\right), \quad W\left(\mathfrak{g}, \mathfrak{a}_{\mathfrak{p}}\right), \quad W=W\left(\mathfrak{g}, \mathfrak{a}_{\mathfrak{q}}\right), \quad N_{K_{e}}\left(\mathfrak{a}_{\mathfrak{p}}\right) \cap N_{K_{e}}\left(\mathfrak{a}_{\mathfrak{q}}\right) .
$$

Moreover, given $P \in \mathcal{P}_{\sigma}^{\min }$ and $H \in \mathfrak{a}_{\mathrm{q}}$, there is a unique element $H_{0} \in \operatorname{cl} \mathfrak{a}_{\mathrm{q}}^{+}(P)$ which is conjugate to $H$ under $W$.

Proof: If $P \in \mathcal{P}_{\sigma}^{\min }$, then $\mathfrak{a}_{\mathrm{q}}^{+}(P)$ is the open positive chamber for the positive system $\Sigma(P)$ of the root system $\Sigma$. Also, $W$ is the Weyl group of $\Sigma$. Thus, the final assertion follows by a well known property of Weyl groups. We turn to the assertions about equivalence of conjugation.

For the first two listed groups the equivalence follows from Lemma 1 in 30, p. 111. For the equivalence for the third group, let $H_{1}, H_{2} \in \mathfrak{a}_{\mathrm{q}}$ and assume that $H_{2}=\varphi\left(H_{1}\right)$ for some $\varphi \in \operatorname{Int}\left(\mathfrak{g}_{\mathrm{c}}\right)$. We may fix $P \in \mathcal{P}_{\sigma}^{\min }$ such that $H_{1} \in \operatorname{cl}\left(\mathfrak{a}_{\mathrm{q}}^{+}(P)\right)$. There exists a $s \in W$ such that $s^{-1}\left(H_{2}\right) \in \operatorname{cl}\left(\mathfrak{a}_{\mathrm{q}}^{+}(P)\right)$. Fix a choice $\Sigma^{+}\left(\mathfrak{g}, \mathfrak{a}_{\mathfrak{p}}\right)$ of positive roots for $\Sigma\left(\mathfrak{g}, \mathfrak{a}_{\mathfrak{p}}\right)$ that is compatible with $\Sigma(P)$, and let $\mathfrak{a}_{\mathfrak{p}}^{+}$be the associated positive chamber. Then $\operatorname{cl}\left(\mathfrak{a}_{\mathfrak{q}}^{+}(P)\right) \subset \operatorname{cl}\left(\mathfrak{a}_{\mathfrak{p}}^{+}\right)$. Since $W$ is naturally isomorphic to $N_{K}\left(\mathfrak{a}_{\mathrm{q}}\right) / Z_{K}\left(\mathfrak{a}_{\mathrm{q}}\right)$, the elements $s^{-1}\left(H_{2}\right)$ and $H_{1}$ are conjugate under $\operatorname{Int}\left(\mathfrak{g}_{\mathfrak{c}}\right)$. Hence, they are already conjugate under $W\left(\mathfrak{g}, \mathfrak{a}_{\mathfrak{p}}\right)$. Being both contained in $\mathrm{cl}\left(\mathfrak{a}_{\mathfrak{p}}^{+}\right)$, the elements must be equal and we conclude that $H_{2}=s\left(H_{1}\right)$. The equivalence for the third group now follows.

Using Corollary 3.2 (b), we immediately obtain the equivalence for the fourth group from the one for the third.

Lemma 3.4 Let $\mathfrak{a}$ be a linear subspace of $\mathfrak{a}_{\mathrm{q}}$ and assume that $\varphi \in \operatorname{Int}\left(\mathfrak{g}_{\mathrm{C}}\right)$ maps $\mathfrak{a}$ into $\mathfrak{a}_{\mathrm{q}}$. Then there exists a $s \in W$ such that $\left.s\right|_{\mathfrak{a}}=\left.\varphi\right|_{\mathfrak{a}}$.

Proof: The proof is identical to the proof of Cor. 2 in [30, p. 112, with use of Lemma 3.3 instead of 30, Lemma 1.

Corollary 3.5 Let $\mathfrak{a}_{1}, \mathfrak{a}_{2}$ be linear subspaces of $\mathfrak{a}_{\mathrm{q}}$, then

$$
W\left(\mathfrak{a}_{2} \mid \mathfrak{a}_{1}\right)=\left\{t \in \operatorname{Hom}\left(\mathfrak{a}_{1}, \mathfrak{a}_{2}\right)|\exists s \in W: \quad t=s|_{\mathfrak{a}_{1}}\right\} .
$$


We briefly interrupt our discussion of Weyl groups to collect some useful facts about conjugacy classes of the parabolic subgroups from $\mathcal{P}_{\sigma}$.

Lemma 3.6 Let $\mathfrak{a}_{\mathfrak{p}}$ be a maximal abelian subspace of $\mathfrak{p}$, containing $\mathfrak{a}_{\mathfrak{q}}$, and let $Q \in \mathcal{P}_{\sigma}$.

(a) There exists a $k \in N_{K_{e}}\left(\mathfrak{a}_{\mathrm{q}}\right) \cap N_{K_{e}}\left(\mathfrak{a}_{\mathfrak{p}}\right)$ such that $k Q k^{-1}$ is standard.

(b) If $F, F^{\prime} \subset \Delta$ are such that $P_{F}$ and $P_{F^{\prime}}$ are conjugate under $G$, then $F=$ $F^{\prime}$.

(c) There exists a unique subset $F \subset \Delta$ such that $Q$ is conjugate to $P_{F}$ under $G$.

(d) If $P \in \mathcal{P}_{\sigma}$ is conjugate to $Q$ under $G$, then it is already conjugate to $Q$ under $N_{K_{e}}\left(\mathfrak{a}_{\mathrm{q}}\right) \cap N_{K_{e}}\left(\mathfrak{a}_{\mathfrak{p}}\right)$.

Proof: There exists a $s \in W$ such that the parabolic equivalence class $s\left(\mathfrak{a}_{Q \mathrm{q}}^{+}\right)$ is contained in $\mathrm{cl} \mathfrak{a}_{\mathrm{q}}^{+}\left(P_{0}\right)$, hence equals $\mathfrak{a}_{F \mathrm{q}}^{+}$, for some $F \subset \Delta$. It follows that $s Q s^{-1}=P_{F}$, see Section 2. Now apply Corollary 3.2 (b) to obtain (a).

For (b), we note that $P_{F}$ and $P_{F^{\prime}}$ both contain the minimal standard $\sigma$ parabolic subgroup $P_{0}$. Hence, $P_{F}=P_{F^{\prime}}$, by 30, p. 111, Lemma 2. This implies that $F=F^{\prime}$, see Section 2 Assertions (c) and (d) both follow from combining (a) and (b).

We end this section with a discussion of automorphisms connecting $\theta$-stable Cartan subspaces of $\mathfrak{q}$; see Section 2 for basic notation.

If $\mathfrak{b}_{1}$ and $\mathfrak{b}_{2}$ are two $\theta$-stable abelian subspaces of $\mathfrak{q}$, then we define

$$
W\left(\mathfrak{b}_{2} \mid \mathfrak{b}_{1}\right):=\left\{\left.\varphi\right|_{\mathfrak{b}_{1 \mathbb{C}}} \mid \varphi \in \operatorname{Int}\left(\mathfrak{g}_{\mathbb{C}}\right), \varphi\left({ }^{\mathrm{d}} \mathfrak{b}_{1}\right) \subset{ }^{\mathrm{d}} \mathfrak{b}_{2}\right\} .
$$

Note that ${ }^{\mathrm{d}} \mathfrak{b}_{2}$ and ${ }^{\mathrm{d}} \mathfrak{b}_{1}$ are abelian subspaces of $\mathfrak{p}^{\mathrm{d}}$. Using the notation of the first part of this section, relative to the algebra $\mathfrak{g}^{\mathrm{d}}=\mathfrak{k}^{\mathrm{d}} \oplus \mathfrak{p}^{\mathrm{d}}$ with the indicated Cartan decomposition, we see that complex linear extension induces a natural isomorphism

$$
W\left({ }^{\mathrm{d}} \mathfrak{b}_{2} \mid{ }^{\mathrm{d}} \mathfrak{b}_{1}\right) \simeq W\left(\mathfrak{b}_{2} \mid \mathfrak{b}_{1}\right) .
$$

In particular, it follows from this that the set in (3.1) is finite. Moreover, if $\mathfrak{b}_{1}$ and $\mathfrak{b}_{2}$ are contained in $\mathfrak{p} \cap \mathfrak{q}$, the notation (3.1) is consistent with the notation introduced earlier in this section.

Lemma 3.7 Let $\mathfrak{b}_{1}$ and $\mathfrak{b}_{2}$ two $\theta$-stable subspaces of a fixed $\theta$-stable Cartan subspace $\mathfrak{d}$ of $\mathfrak{q}$. Then

$$
W\left(\mathfrak{b}_{2} \mid \mathfrak{b}_{1}\right)=\left\{\left.s\right|_{\mathfrak{b}_{1 \mathbb{C}}} \mid s \in W(\mathfrak{d}), \quad s\left({ }^{\mathrm{d}} \mathfrak{b}_{1}\right) \subset{ }^{\mathrm{d}} \mathfrak{b}_{2}\right\} .
$$

Proof: This follows from Corollary 3.5] applied with $\mathfrak{a}_{1}={ }^{d} \mathfrak{b}_{1}, \mathfrak{a}_{2}={ }^{d} \mathfrak{b}_{2}$ and $\mathfrak{a}_{\mathfrak{p}}={ }^{d} \mathfrak{d}$. 
If $s \in W\left(\mathfrak{b}_{2} \mid \mathfrak{b}_{1}\right)$, then by $s^{*}$ we denote the map $\mathfrak{b}_{2 \mathbb{C}}^{*} \rightarrow \mathfrak{b}_{1 \mathbb{C}}^{*}$ given by pullback, i.e.,

$$
s^{*} \nu:=\nu \circ s, \quad\left(\nu \in \mathfrak{b}_{2 \mathrm{C}}^{*}\right) .
$$

If $\mathfrak{b}_{1}, \mathfrak{b}_{2} \subset \mathfrak{q}$ are two $\theta$-stable Cartan subspaces, then ${ }^{\mathrm{d}} \mathfrak{b}_{1}$ and ${ }^{\mathrm{d}} \mathfrak{b}_{2}$ are conjugate under an interior automorphism of $\mathfrak{g}^{d}$ that commutes with $\theta^{\mathrm{d}}$; hence, the set $W\left(\mathfrak{b}_{2} \mid \mathfrak{b}_{1}\right)$ is non-empty and consists of isomorphisms. If $s$ is any isomorphism from this set, we denote its natural extension to the symmetric algebras by $s$ as well. This extension maps $I\left(\mathfrak{b}_{1}\right)$ into $I\left(\mathfrak{b}_{2}\right)$.

Lemma 3.8 Let $\mathfrak{b}_{1}, \mathfrak{b}_{2}$ be $\theta$-stable Cartan subspaces of $\mathfrak{q}$. Then $W\left(\mathfrak{b}_{2} \mid \mathfrak{b}_{1}\right) \neq \emptyset$. Moreover, if $s \in W\left(\mathfrak{b}_{2} \mid \mathfrak{b}_{1}\right)$, then

$$
s \circ \gamma_{\mathfrak{b}_{1}}=\gamma_{\mathfrak{b}_{2}} .
$$

Proof: The first assertion follows from the discussion preceding the lemma.

Let $K^{d}$ be the analytic subgroup of $\operatorname{Int}\left(\mathfrak{g}_{\mathrm{C}}\right)$ generated by $e^{\text {adk }^{d}}$. Then by Lemma 3.1 applied to ${ }^{\mathrm{d}} \mathfrak{b}_{1},{ }^{\mathrm{d}} \mathfrak{b}_{2} \subset \mathfrak{p}^{\mathrm{d}}$, there exists an element $k \in K^{d}$ such that $s=\left.k\right|_{d_{\mathfrak{b}_{1}}}$. The action of $k$ induces the identity on $U\left(\mathfrak{g}^{d}\right)^{\mathfrak{k}^{d}} / U\left(\mathfrak{k}^{d}\right)^{\mathfrak{k}^{d}} \cap U\left(\mathfrak{g}^{d}\right) \mathfrak{k}^{d}$. Hence, if $D$ belongs to the latter algebra, then $s \gamma_{\mathfrak{b}_{1}}(D)=k \gamma_{\mathfrak{b}_{1}}\left(k^{-1} \cdot D\right)=$ $\gamma_{\mathfrak{b}_{2}}(D)$.

\section{Laurent functionals and operators}

In this section we briefly recall the concept of Laurent functional, introduced in 14]; meanwhile, we fix notation that will be used in the rest of the paper. For details we refer to Sections 10 and 11 of 14 .

Let $V$ be a finite dimensional real linear space, equipped with a positive definite inner product $\langle\cdot, \cdot\rangle$. Its complexification $V_{\mathbb{C}}$ is equipped with the complex bilinear extension of the inner product. We write $P(V)$ for the algebra of polynomial functions $V_{\mathbb{C}} \rightarrow \mathbb{C}$, and $S(V)$ for the symmetric algebra of $V_{\mathbb{C}}$. We identify the latter algebra with the algebra of translation invariant differential operators on $V$, which in turn is identified with the algebra of translation invariant holomorphic differential operators on $V_{\mathrm{c}}$. In both settings, $u \in V$ is identified with the differential operator $f \mapsto d f(\cdot) u$.

Let $X$ be a finite subset of non-zero elements of $V$. By an $X$-hyperplane in $V_{\mathbb{C}}$ we mean an affine hyperplane of the form $H=a+\xi_{\mathbb{C}}^{\perp}$, with $a \in V_{\mathbb{C}}$ and $\xi \in X$. The hyperplane $H$ is said to be real if $a$ may be chosen in $V$. By

$$
\Pi_{X}(V)
$$

we denote the collection of polynomial functions $p \in P\left(V_{\mathrm{c}}\right)$ with zero locus $p^{-1}(0)$ equal to a finite union of $X$-hyperplanes. The subset consisting of $p$ with zero locus a finite union of real $X$-hyperplanes is denoted by $\Pi_{X, \mathbb{R}}(V)$. Note that $\Pi_{X}(V)$ consists of all polynomial functions that may be written as a non-zero multiple of a product of factors of the form $\langle\xi, \cdot\rangle-c$, with $\xi \in X$ and $c \in \mathbb{C}$. The subset $\Pi_{X, \mathbb{R}}(V)$ consists of such products with $c \in \mathbb{R}$ in all factors. 
By an $X$-configuration in $V_{\mathbb{C}}$ we mean a locally finite collection of $X$-hyperplanes in $V_{\mathbb{C}}$. The configuration is said to be real if all its hyperplanes are real. If $a \in V_{\mathbb{C}}$, then by $\mathcal{M}\left(V_{\mathbb{C}}, a, X\right)$ we denote the space of germs at $a$ of meromorphic functions with singular locus contained in the union of the hyperplanes $a+\xi_{\mathbb{C}}^{\perp}$, for $\xi \in X$. Let $\mathbb{N}^{X}$ denote the space of maps $X \rightarrow \mathbb{N}$. For $d \in \mathbb{N}^{X}$ we define the polynomial function $\pi_{a, d} \in \Pi_{X}(V)$ by

$$
\pi_{a, d}(z)=\prod_{\xi \in X}\langle\xi, z-a\rangle^{d} .
$$

Let $\mathcal{O}_{a}\left(V_{\mathbb{C}}\right)$ denote the space of germs of holomorphic functions at $a$. Then $\mathcal{M}\left(V_{\mathbb{C}}, a, X\right)$ is the union of the spaces $\pi_{a, d}^{-1} \mathcal{O}_{a}\left(V_{\mathbb{C}}\right)$ for $d \in \mathbb{N}^{X}$. The space $\mathcal{M}\left(V_{\mathbb{C}}, a, X\right)_{\text {laur }}^{*}$ of $X$-Laurent functionals at $a$ is defined as the subspace of $\mathcal{M}\left(V_{\mathbb{C}}, a, X\right)^{*}$ consisting of $\mathcal{L}$ with the property that for every $d \in \mathbb{N}^{X}$ there exists a $u_{\mathcal{L}, d} \in S(V)$ such that

$$
\mathcal{L} \varphi=\left[u_{\mathcal{L}, d} \pi_{a, d} \varphi\right](a), \quad \text { for all } \quad \varphi \in \pi_{a, d}^{-1} \mathcal{O}_{a}\left(V_{\mathbb{C}}\right) .
$$

The element $u_{\mathcal{L}}$ belongs to a projective limit space $S_{\leftarrow}(V, X)$ whose definition is suggested by the above, see [14, Sect. 10, for more details. Moreover, the map $\mathcal{L} \mapsto u_{\mathcal{L}}$ defines a linear isomorphism

$$
\mathcal{M}\left(V_{\mathbb{C}}, a, X\right)_{\text {laur }}^{*} \stackrel{\simeq}{\longrightarrow} S_{\leftarrow}(V, X),
$$

see [14, Lemma 10.4.

The space on the left-hand side of the above isomorphism only depends on $X$ through its proportionality class. More precisely, a finite set $X^{\prime} \subset V \backslash\{0\}$ is said to be proportional to $X$ if every element of one of the sets $X, X^{\prime}$ is proportional to an element of the other set. If $X$ and $X^{\prime}$ are proportional sets, then $\mathcal{M}\left(V_{\mathrm{c}}, a, X\right)_{\text {laur }}^{*}=\mathcal{M}\left(V_{\mathbb{C}}, a, X^{\prime}\right)_{\text {laur }}^{*}$, see [14], Lemma 10.3.

If $\Omega \subset V_{\mathbb{C}}$ is open and $E$ a complete locally convex space, then a (densely defined) $E$-valued function $f$ on $\Omega$ is said to be meromorphic if for every $z_{0} \in \Omega$ there exists an open neighborhood $\Omega_{0}$ of $z_{0}$ and a holomorphic function $g \in$ $\mathcal{O}\left(\Omega_{0}\right)$ such that $\left.g f\right|_{\Omega_{0}}$ is a holomorphic $E$-valued function on $\Omega$. The space of $E$-valued meromorphic functions on $\Omega$ is denoted by $\mathcal{M}(\Omega, E)$. A point $z \in \Omega$ is said to be a regular point of $f \in \mathcal{M}(\Omega, E)$ if $f$ is holomorphic in a neighborhood of $z$. The collection of regular points of $f$ is denoted by reg $f$.

Let $\mathcal{H}$ be an $X$-configuration in $V_{\mathbb{C}}$. By $\mathcal{M}\left(V_{\mathbb{C}}, \mathcal{H}, E\right)$ we denote the space of meromorphic functions $V_{\mathrm{C}} \rightarrow E$ with singular locus contained in $\cup \mathcal{H}$. We agree to write $\mathcal{M}\left(V_{\mathbb{c}}, \mathcal{H}\right):=\mathcal{M}\left(V_{\mathbb{c}}, \mathcal{H}, \mathbb{C}\right)$.

The space $\mathcal{M}\left(V_{\mathrm{c}}, \mathcal{H}, E\right)$ is topologized as follows. Let $X^{0} \subset X$ be minimal subject to the condition that $X^{0}$ and $X$ are proportional. For each $X$-hyperplane $H \subset V_{\mathbb{C}}$ there exists a unique $\alpha_{H} \in X^{0}$ and a unique first order polynomial function $l_{H}$ of the form $z \mapsto\left\langle\alpha_{H}, z\right\rangle-c$, with $c \in \mathbb{C}$, such that $H=l_{H}^{-1}(0)$.

We denote by $\mathbb{N}^{\mathcal{H}}$ the space of maps $\mathcal{H} \rightarrow \mathbb{N}$. For $d \in \mathbb{N}^{\mathcal{H}}$ and $\omega \subset V_{\mathbb{C}}$ a bounded subset, we define the polynomial function $\pi_{\omega, d} \in \Pi_{X}(V)$ by

$$
\pi_{\omega, d}=\prod_{\substack{H \in \mathcal{H} \\ H \cap \omega \neq \emptyset}} l_{H}^{d(H)} .
$$


A change of choice of $X^{0}$ only causes a change of this polynomial by a nonzero factor. If $E$ is a complete locally convex space, we define $\mathcal{M}\left(V_{\mathbb{C}}, \mathcal{H}, d, E\right)$ to be the space of meromorphic $E$-valued functions $\varphi$ on $V_{\mathbb{C}}$ with the property that $\pi_{\omega, d} \varphi$ is holomorphic on $\omega$, for every bounded open subset $\omega \subset V_{\mathbb{C}}$. This space is equipped with the weakest locally convex topology that makes every map $\left.\varphi \mapsto \pi_{\omega, d} \varphi\right|_{\omega}$ continuous from $\mathcal{M}\left(V_{\mathbb{C}}, \mathcal{H}, d, E\right)$ to $\mathcal{O}(\omega, E)$. This topology is complete; it is Fréchet if $E$ is. We equip $\mathbb{N}^{\mathcal{H}}$ with the partial ordering $\preceq$ defined by $d \preceq d^{\prime} \Longleftrightarrow \forall H \in \mathcal{H}: d(H) \leq d^{\prime}(H)$. We now have

$$
\mathcal{M}\left(V_{\mathbb{C}}, \mathcal{H}, E\right)=\cup_{d \in N^{\mathcal{H}}} \mathcal{M}\left(V_{\mathbb{C}}, \mathcal{H}, d, E\right)
$$

Accordingly, we equip the space on the left-hand side with the direct limit locally convex topology.

Any non-empty intersection of $X$-hyperplanes in $V_{\mathbb{C}}$ is called an $X$-subspace of $V_{\mathbb{C}}$. An $X$-subspace $L$ of $V_{\mathrm{C}}$ may be written as $L=a+V_{L \mathbb{C}}$, with $V_{L} \subset V$ a real linear subspace. Let $V_{L \mathbb{C}}^{\perp}$ denote the complexification of its orthocomplement. The intersection $V_{L \mathbb{C}}^{\perp} \cap L$ consists of a single point $c(L)$, called the central point of $L$. Via the translation $x \mapsto c(L)+x$ from $V_{L \mathbb{C}}$ onto $L$ we equip $L$ with the structure of a complex linear space, together with a real form with a positive definite inner product on it.

If $\mathcal{H}$ is an $X$-configuration and $L \subset V$ an $X$-affine subspace, we define $\mathcal{H}_{L}$ to be the collection of affine hyperplanes in $L$ of the form $H \cap L$, with $H \in \mathcal{H}$ a hyperplane that properly intersects $L$. Let $X(L):=X \cap V_{L}^{\perp}$ and let $X_{r} \subset V_{L}$ be the image of $X \backslash X(L)$ under the orthogonal projection onto $V_{L}$. The image of $X_{r}$ in $L$ under translation by $c(L)$ is denoted by $X_{L}$. Thus, $\left(L, X_{L}\right)$ is the analogue of $\left(V_{\mathbb{C}}, X\right)$. The collection $\mathcal{H}_{L}$ is a $X_{L}$-configuration in $L$.

If $\mathcal{L}$ is a Laurent functional in $\mathcal{M}\left(V_{L \mathbb{C}}^{\perp}, 0, X(L)\right)_{\text {laur }}^{*}$, then $\mathcal{L}$ induces a continuous linear map

$$
\mathcal{L}_{*}: \mathcal{M}\left(V_{\mathbb{C}}, \mathcal{H}\right) \rightarrow \mathcal{M}\left(L, \mathcal{H}_{L}\right),
$$

given by the formula

$$
\mathcal{L}_{*} \varphi(\nu):=\mathcal{L}[\varphi(\cdot+\nu)],
$$

for $\varphi \in \mathcal{M}\left(V_{\mathbb{\mathbb { C }}}, \mathcal{H}\right)$ and generic $\nu \in L$. The map $\mathcal{L}_{*}$ belongs to the space Laur $\left(V_{\mathbb{C}}, L, \mathcal{H}\right)$ of Laurent operators $\mathcal{M}\left(V_{\mathbb{c}}, \mathcal{H}\right) \rightarrow \mathcal{M}\left(L, \mathcal{H}_{L}\right)$, as defined in [1], Sect. 1.3, see also 14, Sect. 11. It follows from the definition of Laurent operator combined with the isomorphism $\mathcal{M}\left(V_{L \mathbb{C}}^{\perp}, 0, X(L)\right)_{\text {laur }}^{*} \simeq S_{\leftarrow}\left(V_{L}^{\perp}, X(L)\right)$ given by (4.2) that the map $\mathcal{L} \mapsto \mathcal{L}_{*}$ defines a linear surjection

$$
\mathcal{M}\left(V_{L}, X(L), 0\right)_{\text {laur }}^{*} \rightarrow \operatorname{Laur}\left(V_{\mathbb{C}}, L, \mathcal{H}\right) \rightarrow 0 .
$$

Accordingly, a Laurent operator may alternatively be defined as any continuous linear operator $\mathcal{M}\left(V_{\mathbb{C}}, \mathcal{H}\right) \rightarrow \mathcal{M}\left(L, \mathcal{H}_{L}\right)$ of the form $\mathcal{L}_{*}$ with $\mathcal{L}$ a Laurent functional from the space on the left-hand side of (4.5).

More generally, if $\mathcal{L} \in \mathcal{M}\left(V_{L}, X(L), 0\right)_{\text {laur }}^{*}$ and if $E$ is a complete locally convex space then the algebraic tensor product $\mathcal{L}_{*} \otimes I_{E}$ has a unique extension to a continuous linear map $\mathcal{M}\left(V_{\mathbb{C}}, \mathcal{H}, E\right) \rightarrow \mathcal{M}\left(L, \mathcal{H}_{L}, E\right)$ that we briefly denote by $\mathcal{L}_{*}$ again. 
The concept of Laurent functional may be extended as follows, see 14, Def. 10.8. Let $\mathcal{M}\left(V_{\mathrm{C}}, *, X\right)_{\text {laur }}^{*}$ be the disjoint union of the spaces $\mathcal{M}\left(V_{\mathbb{C}}, a, X\right)_{\text {laur }}^{*}$, for $a \in V_{\mathbb{C}}$. An $X$-Laurent functional on $V_{\mathbb{C}}$ is defined to be a finitely supported section of $\mathcal{M}\left(V_{\mathbb{C}}, *, X\right)_{\text {laur }}^{*}$, i.e., a map $\mathcal{L}: V_{\mathbb{C}} \rightarrow \mathcal{M}\left(V_{\mathbb{C}}, *, X\right)_{\text {laur }}^{*}$ with $\mathcal{L}_{a} \in \mathcal{M}\left(V_{\mathbb{C}}, a, X\right)_{\text {laur }}^{*}$ for every $a \in V_{\mathbb{C}}$ and with $\operatorname{supp} \mathcal{L}:=\left\{a \in V_{\mathbb{C}} \mid \mathcal{L}_{a} \neq 0\right\}$ a finite set. The set of all $X$-Laurent functionals on $V_{\mathbb{C}}$ naturally forms a complex linear space, denoted $\mathcal{M}\left(V_{\mathrm{c}}, X\right)_{\text {laur }}^{*}$.

Let $\mathcal{H}, L$ be as before. If $a \in V_{\mathbb{C}}$ we denote by $\mathcal{H}_{L}(a)$ the $X_{L}$-configuration consisting of all hyperplanes $H^{\prime}$ in $L$ for which there exists a $H \in \mathcal{H}$ such that $H^{\prime}=L \cap[(-a)+H]$. If $S \subset V_{L \mathbb{C}}^{\perp}$ is a finite subset, we put

$$
\mathcal{H}_{L}(S)=\cup_{a \in S} \mathcal{H}_{L}(a) .
$$

Let now $\mathcal{L} \in \mathcal{M}\left(V_{\mathrm{C}}^{\perp}, X\right)_{\text {laur }}^{*}$ and put $S=\operatorname{supp} \mathcal{L}$. Then from the above discussion it follows in a straightforward manner that the formula (4.4) defines a continuous linear map $\mathcal{L}_{*}: \mathcal{M}\left(V_{\mathbb{C}}, \mathcal{H}\right) \rightarrow \mathcal{M}\left(L, \mathcal{H}_{L}(S)\right)$. As above, if $E$ is a complete locally convex space, then the tensor product map $\mathcal{L}_{*} \otimes I_{E}$ has a unique extension to a continuous linear map

$$
\mathcal{L}_{*}: \mathcal{M}\left(V_{\mathbb{C}}, \mathcal{H}, E\right) \rightarrow \mathcal{M}\left(L, \mathcal{H}_{L}(S), E\right)
$$

see 14], Cor. 11.6.

By $\mathcal{M}\left(V_{\mathbb{C}}, X, E\right)$ we denote the space of meromorphic $E$-valued functions on $V_{\mathbb{C}}$ with singular locus contained in the union of an $X$-configuration. Every Laurent functional $\mathcal{L} \in \mathcal{M}\left(V_{L \mathbb{C}}^{\perp}, X(L)\right)_{\text {laur }}^{*}$ determines a unique continuous linear map $\mathcal{L}_{*}: \mathcal{M}\left(V_{\mathbb{C}}, X, E\right) \rightarrow \mathcal{M}\left(L, X_{L}, E\right)$ such that $\mathcal{L}_{*}$ restricts to the map (4.7) for every $X$-configuration $\mathcal{H}$ in $V_{\mathbb{C}}$. See [14, Lemma 11.8, for details.

\section{$5 \quad$ Normalization of residues and measures}

For the explicit determination of the constants in the Plancherel formula, it is of importance to specify the precise normalizations of residual operators and measures that will be used in the rest of this paper.

Let $P_{0}$ be the standard parabolic subgroup in $\mathcal{P}_{\sigma}^{\min }$ and let $t \in \mathrm{WT}(\Sigma)$ be a $W$-invariant residue weight, see $\S 2$ Let $b$ be a $W$-invariant positive definite inner product on $\mathfrak{a}_{\mathrm{q}}^{*}$. Associated with the data $\Sigma^{+}, t, b$, we defined in [11, beginning of $\S 3.4$, for each subset $F \subset \Delta$ and every element $\lambda \in{ }^{*} \mathfrak{a}_{F \mathrm{q}}^{*}$, a universal residue operator

$$
\operatorname{Res}_{\lambda+\mathfrak{a}_{F q}^{*}}^{t}:=\operatorname{Res}_{\lambda+\mathfrak{a}_{F q}^{*}}^{P_{0}, t},
$$

which encodes the procedure of taking a residue along the affine subspace $\lambda+$ $\mathfrak{a}_{F \mathrm{qC}}^{*}$ of $\mathfrak{a}_{\mathrm{qC}}^{*}$. In [11], text below Eqn. (3.6), this residue operator is introduced as an element of a project limit space $S_{\leftarrow}\left({ }^{*} \mathfrak{a}_{F \mathrm{q}}^{*}, \bar{\Sigma}_{F}^{+}\right)$, defined in [1], $\S 1.3$; here $\bar{\Sigma}_{F}^{+}$denotes the collection of indivisible roots in $\Sigma^{+} \cap{ }^{*} \mathfrak{a}_{F \mathrm{q}}^{*}$. However, to make the residue operator into an object as canonical as possible, we shall prefer to view it as a Laurent functional. 
Applying the results of Section 4 with $V={ }^{*} \mathfrak{a}_{F \mathrm{q}}^{*}, X=\Sigma_{F}, X^{0}=\bar{\Sigma}_{F}^{+}$and $a=0$, we obtain an isomorphism

$$
\mathcal{M}\left({ }^{*} \mathfrak{a}_{F \mathrm{qC}}^{*}, 0, \Sigma_{F}\right)_{\text {laur }}^{*} \simeq S_{\leftarrow}\left({ }^{*} \mathfrak{a}_{F \mathrm{q}}^{*}, \bar{\Sigma}_{F}^{+}\right) .
$$

In the present paper, the universal residue operator (5.1) is accordingly viewed as an element of the space $\mathcal{M}\left({ }^{*} \mathfrak{a}_{F \mathrm{qc}}^{*}, 0, \Sigma_{F}\right)_{\text {laur }}^{*}$ of $\Sigma_{F}$-Laurent functionals at the origin in ${ }^{*} \mathfrak{a}_{F q \mathrm{c}}^{*}$.

By Eqn. [4.5), with $V=\mathfrak{a}_{\mathrm{q}}^{*}, X=\Sigma$ and $L=\lambda+\mathfrak{a}_{F \mathrm{q}}^{*}$, we see that $\mathcal{L} \mapsto$ $\mathcal{L}_{*}$ induces a surjective linear map from $\mathcal{M}\left({ }^{*} \mathfrak{a}_{F \mathrm{qC}}^{*}, \Sigma_{F}\right)_{\text {laur }}^{*}$ onto Laur $\left(\mathfrak{a}_{\mathrm{qC}}^{*}, \lambda+\right.$ $\left.\mathfrak{a}_{F q \mathbb{C}}^{*}, \mathcal{H}\right)$. In this context we omit the star in the notation, and use the notation (5.1) also for the Laurent operator defined by the universal residue operator. Thus, (4.4) becomes

$$
\left(\operatorname{Res}_{\lambda+\mathfrak{a}_{F q}^{*}}^{t} \varphi\right)(\nu)=\operatorname{Res}_{\lambda+\mathfrak{a}_{F q}^{*}}^{t}[\varphi(\cdot+\nu)]
$$

for every $\varphi \in \mathcal{M}\left(\mathfrak{a}_{\mathrm{qC}}^{*}, \mathcal{H}\right)$ and $\nu \in \mathfrak{a}_{F \mathrm{qC}}^{*}$ generic. In this way the notation becomes compatible with the notation of [11].

All definitions in [11] are given with reference to the fixed $W$-invariant inner product $b$ on $\mathfrak{a}_{\mathrm{q}}^{*}$, denoted $\langle\cdot, \cdot\rangle$ in [11, so that a priori the universal residue operator depends on the particular choice of the inner product. However, as we will show, the dependence is through certain measures determined by $b$. To explain this, it is convenient to first introduce some general terminology.

If $\mathfrak{v}$ is a real finite dimensional vector space, let ${ }^{\circ} \wedge(\mathfrak{v})$ denote the one dimensional real linear space of densities on $\mathfrak{v}$, i.e., the space of maps $\omega: \mathfrak{v}^{n} \rightarrow \mathbb{R}$, where $n=\operatorname{dim} \mathfrak{v}$, transforming according to the rule $\omega \circ A^{n}=|\operatorname{det} A| \omega$, for all $A \in \operatorname{End}(\mathfrak{v})$. Evaluation at the origin induces a natural isomorphism from the space of translation invariant densities on $\mathfrak{v}$, where $\mathfrak{v}$ is viewed as a manifold, onto ${ }^{\wedge}(\mathfrak{v})$; we shall identify accordingly. Consequently, via integration the space ${ }^{\circ} \wedge(\mathfrak{v})$ may be identified with the space of Radon measures $\mathbb{R} d \lambda$, where $d \lambda$ is a choice of Lebesgue measure on $\mathfrak{v}$. If $\mathfrak{v}$ is equipped with a positive definite inner product, then by the normalized density on $\mathfrak{v}$ we mean the unique element $\omega \in{ }^{\circ} \wedge(\mathfrak{v})$ such that $\omega\left(e_{1}, \ldots, e_{n}\right)=1$, for every orthonormal basis $e_{1}, \ldots, e_{n}$ of $\mathfrak{v}$.

We shall often encounter the situation that $\mathfrak{v}=i \mathfrak{b}$ with $\mathfrak{b}$ a subspace of a real linear space $V$; here multiplication by $i$ is defined in the complexification $V_{\mathbb{C}}$ of $V$. If $V$ comes equipped with a positive definite inner product $\langle\cdot, \cdot\rangle$, we extend it to $V_{\mathbb{C}}$ by complex bilinearity, and equip $i \mathfrak{b}$ with the positive definite inner product $-\langle\cdot, \cdot\rangle$. Accordingly, in this setting it makes sense to speak of the normalized density on $i \mathfrak{b}$.

Let $d \mu \in{ }^{\circ} \wedge(i \mathfrak{b})$. If $\lambda \in V_{\mathbb{C}}$, then we shall adopt the convention to also denote by $d \mu$ the density on the real affine subspace $\lambda+i \mathfrak{b}$ of $V_{\mathbb{C}}$, obtained by transportation under the translation $X \mapsto \lambda+X, i \mathfrak{b} \rightarrow \lambda+i \mathfrak{b}$. Accordingly, by unoriented integration, the density $d \mu$ determines a real Radon measure on $\lambda+i \mathfrak{b}$, which we shall denote by the same symbol.

We now return to the dependence of the residue operator (5.1) on the choice of $b$. For every $\alpha \in \Sigma$, the orthogonal reflection $s_{\alpha}$ is independent of the particular choice of $b$, and therefore so are the root hyperplanes $\alpha^{\perp}$, and, more 
generally, the root spaces $\mathfrak{a}_{F \mathrm{q}}^{*}$ and their orthocomplements ${ }^{*} \mathfrak{a}_{F \mathrm{q}}^{*}$, for $F \subset \Delta$. Combining this observation with the uniqueness statement of 11 , Theorem 1.13, it follows that the residue operator $\operatorname{Res}_{\lambda+\mathfrak{a}_{F \mathrm{q}}^{*}}^{t}$ can be completely defined in terms of the data $\Sigma^{+}, t, \lambda+\mathfrak{a}_{F \mathrm{q}}^{*}$ and $b$; moreover, it depends on the latter datum through the quotient measure on $i^{*} \mathfrak{a}_{F \mathrm{q}}^{*}$ of the normalized Lebesgue measures $d \lambda$ on $i \mathfrak{a}_{\mathrm{q}}^{*}$ and $d \mu_{F}$ on $i \mathfrak{a}_{F \mathrm{q}}^{*}$.

To keep track of constants coming from comparing residue operators related to different choices of the mentioned inner product we shall introduce a version of the residue operator (5.1) that is independent of the choice of $b$.

The unnormalized residue operator is defined as the unique Laurent functional

$$
\underline{\operatorname{Res}}_{\lambda+\mathfrak{a}_{F \mathrm{q}}^{*}}^{t} \in \mathcal{M}\left({ }^{*} \mathfrak{a}_{F \mathrm{qC}}^{*}, 0, \Sigma_{F}\right)_{\text {laur }}^{*} \otimes \operatorname{Hom}\left({ }^{\circ} \wedge\left(i \mathfrak{a}_{\mathrm{q}}^{*}\right),{ }^{\circ} \wedge\left(i \mathfrak{a}_{F \mathrm{q}}^{*}\right)\right)
$$

satisfying the following requirement. Let (5.1) be defined relative to the given choice of $b$ and let $d \lambda \in{ }^{\circ} \wedge\left(i \mathfrak{a}_{\mathrm{q}}^{*}\right)$ and $d \mu_{F} \in{ }^{\circ} \wedge\left(i \mathfrak{a}_{F \mathrm{q}}^{*}\right)$ be the normalized densities associated with $b$. Then the requirement is that

$$
\underline{\operatorname{Res}}_{\lambda+\mathfrak{a}_{F \mathrm{q}}^{*}}^{t}(\varphi)(d \lambda)=\operatorname{Res}_{\lambda+\mathfrak{a}_{F \mathrm{q}}^{*}}^{t}(\varphi) d \mu_{F},
$$

for $\varphi \in \mathcal{M}\left({ }^{*} \mathfrak{a}_{F \mathrm{qC}}^{*}, 0, \Sigma_{F}\right)$. From the above mentioned dependence of (5.1) on $b$ through the quotient density on $i^{*} \mathfrak{a}_{F \mathrm{q}}^{*}$ of $d \lambda$ and $d \mu_{F}$, it follows that the residue operator in (5.3) only depends on the data $\Sigma^{+}, t, F, \lambda$ and not on the choice of $b$. In other words, if (5.1), $d \lambda$ and $d \mu_{F}$ had been defined relative to an arbitrary $W$-invariant inner product on $\mathfrak{a}_{\mathrm{q}}$, then formula (5.4) would be valid as well.

Suppose now that for each $F \subset \Delta$ a non-trivial density $d \mu_{F} \in{ }^{\circ} \wedge\left(i \mathfrak{a}_{F q}^{*}\right)$ is given. In particular, $d \lambda:=d \mu_{\emptyset}$ is given. Then we can use the formula (5.4) to define residual operators $\operatorname{Res}_{\lambda+\mathfrak{a}_{F q}^{*}}^{t}$. With this definition, the integral formula of 11, Thm. 3.16, is valid.

In the rest of this paper, we fix a choice $d x$ of invariant measure on $\mathrm{X}$. In the rest of this section we will describe how this choice determines all other choices of normalization of measures, and, by the preceding discussion, all choices of normalization of residual operators.

As in [10], $\S 3$, the measure $d x$ determines a choice $d a$ of Haar measure on $A_{\mathrm{q}}$, and a choice $d \lambda$ of Lebesgue measure on $i \mathfrak{a}_{\mathrm{q}}^{*}$. A change of the measure $d x$ by a multiplication by a positive factor $c$ causes a change of $d a$ by the same factor. This in turn causes a change of the measure $d \lambda$ by the factor $c^{-1}$. It follows that the product measure $d x d \lambda$ on $\mathrm{X} \times i \mathfrak{a}_{\mathrm{q}}^{*}$ is independent of the particular choice of the measure $d x$.

In order to be able to use the formula (5.2) of [12], we normalize the Lebesgue measures $d \mu_{F}$ of $i \mathfrak{a}_{F q}^{*}$, for $F \subset \Delta$, as in the text following the mentioned formula. We describe this normalization in a somewhat more general setting, in terms of the above terminology. Let $B$ be the bilinear form of $\mathfrak{g}$, fixed in the beginning of Section 2 Via restriction and dualization, $B$ induces a positive definite inner product on $\mathfrak{a}_{\mathrm{q}}^{*}$, which we denote by $B$ as well. Let $c>0$ be the positive constant such that $d \lambda$ corresponds to the density on $i \mathfrak{a}_{\mathrm{q}}^{*}$, normalized relative to the inner product $c B$. 
If $P \in \mathcal{P}_{\sigma}$, then $d \mu_{P}$ denotes the Lebesgue measure on $i \mathfrak{a}_{P \mathrm{q}}^{*}$ normalized with respect to $c B$. If $\mathfrak{a}_{G q}^{*}=\{0\}$, which occurs if and only if $G$ has a compact center modulo $H$, we agree that $d \mu_{G}$ has total volume 1 , in accordance with [12, text following (5.2).

The residual operators $\operatorname{Res}_{\lambda+\mathfrak{a}_{F q}^{*}}^{t}$ are now normalized by (5.4) and with respect to the choices of normalizations of measures made. All results of [1] and $[12$ needed in this paper are valid with the normalization of measures and residual operators just described.

If $P \in \mathcal{P}_{\sigma}$, we denote by $d \lambda_{P}$ the choice of Lebesgue measure on $i^{*} \mathfrak{a}_{P \mathrm{q}}^{*}$ for which

$$
d \lambda=d \lambda_{P} d \mu_{P} .
$$

If $v \in{ }^{P} \mathcal{W}$, then by the above discussion of the normalization of $d \lambda$, applied to the space $\mathrm{X}_{P, v}$, a choice $d x_{P, v}$ of invariant measure on $\mathrm{X}_{P, v}$ corresponds in oneto-one fashion with a choice of Lebesgue measure $d \lambda_{P, v}$ on $i^{*} \mathfrak{a}_{P q}^{*}$. Throughout this paper we agree to select $d x_{P, v}$ so that $d \lambda_{P, v}=d \lambda_{P}$.

We end this section with the observation that for $P, Q \in \mathcal{P}_{\sigma}$ with $\mathfrak{a}_{P \mathrm{q}}$ and $\mathfrak{a}_{Q \mathrm{q}}$ conjugate under $W$, the measures $d \mu_{P}$ and $d \mu_{Q}$ are $W$-conjugate. Indeed, this follows from the $W$-invariance of the inner product $B$. From (5.5) we see that the measures $d \lambda_{P}$ and $d \lambda_{Q}$ are $W$-conjugate as well.

\section{A vanishing theorem}

Let $Q \in \mathcal{P}_{\sigma}$. Throughout this paper, we assume $\left(\tau, V_{\tau}\right)$ to be a finite dimensional unitary representation of $K$. In this section we introduce a space $\mathcal{E}_{Q}^{\text {hyp }}(\mathrm{X}: \tau)$ of meromorphic families of $\mathbb{D}(\mathrm{X})$-finite $\tau$-spherical functions and show that the vanishing theorem of [14] applies to it.

Let $\mathfrak{a}_{\mathrm{q}}^{\text {reg }}$ denote the set of regular elements in $\mathfrak{a}_{\mathrm{q}}$ for the root system $\Sigma$ and put $A_{\mathrm{q}}^{\text {reg }}:=\exp \mathfrak{a}_{\mathrm{q}}^{\text {reg. }}$. We define a subset of X by

$$
\mathrm{X}_{+}:=K A_{\mathrm{q}}^{\mathrm{reg}} H
$$

According to [14, Sect. 2, this set is open dense in X. Let $\mathcal{W} \subset N_{K}\left(\mathfrak{a}_{\mathrm{q}}\right)$ be a choice of representatives for $W / W_{K \cap H}$. Then, for each $P \in \mathcal{P}_{\sigma}^{\min }$,

$$
\mathrm{X}_{+}=\cup_{v \in \mathcal{W}} K A_{P \mathrm{q}}^{+} v H \quad \text { (disjoint union). }
$$

By $C^{\infty}\left(\mathrm{X}_{+}: \tau\right)$ we denote the space of smooth functions $f: \mathrm{X}_{+} \rightarrow V_{\tau}$ that are $\tau$-spherical, i.e.,

$$
f(k x)=\tau(k) f(x) \quad\left(x \in \mathrm{X}_{+}, k \in K\right) .
$$

By $\mathcal{A}\left(\mathrm{X}_{+}: \tau\right)$ we denote the subspace of $f \in C^{\infty}\left(\mathrm{X}_{+}: \tau\right)$ that behave finitely under the action of the algebra $\mathbb{D}(\mathrm{X})$. Moreover, we denote the subspaces of these spaces consisting of functions that extend smoothly from $\mathrm{X}_{+}$to all of $\mathrm{X}$ by $C^{\infty}(\mathrm{X}: \tau)$ and $\mathcal{A}(\mathrm{X}: \tau)$, respectively. 
Let $P \in \mathcal{P}_{\sigma}$ and $v \in N_{K}\left(\mathfrak{a}_{\mathrm{q}}\right)$. We put $K_{P}:=K \cap M_{P}$ and define $\mathrm{X}_{P, v,+}$ to be the analogue of the set $\mathrm{X}_{+}$for the triple $\left(M_{P}, K_{P}, M_{P} \cap v H v^{-1}\right)$. In particular, $\mathrm{X}_{P, v,+}$ is an open dense subset of $\mathrm{X}_{P, v}$.

We define the function $\left.R_{P, v}: M_{1 P} \rightarrow\right] 0, \infty$ [ as in [14], Section 3. According to [14, Lemma 3.2, this function is left $K_{P^{-}}$and right $M_{1 P} \cap v H v^{-1}$-invariant. Moreover, if $P \neq G, a \in A_{\mathrm{q}}$ and $u \in N_{K_{P}}\left(\mathfrak{a}_{\mathrm{q}}\right)$, then

$$
R_{P, v}(a u)=\max _{\alpha \in \Sigma(P)} a^{-\alpha} .
$$

Finally, $R_{P, v} \geq 1$ on $\mathrm{X}_{P, v}$. The function $R_{P, v}$ is of importance for the description of a domain of convergence for the series expansion that describes the asymptotic behavior of a function from $\mathcal{A}\left(\mathrm{X}_{+}: \tau\right)$ along $(P, v)$. To be more precise, we define, for $0<r<1$,

$$
A_{P \mathrm{q}}^{+}(r):=\left\{a \in A_{P \mathrm{q}} \mid \forall \alpha \in \Delta_{r}(P): a^{-\alpha}<r\right\} .
$$

Then the following property, see [14, Lemma 3.3, is relevant for the mentioned description of the domain of convergence. For $m \in \mathrm{X}_{P, v}$ and $a \in A_{P \mathrm{q}}$,

$$
m \in \mathrm{X}_{P, v,+}, \quad a \in A_{P \mathrm{q}}^{+}\left(R_{P, v}(m)^{-1}\right) \Rightarrow m a v H \in \mathrm{X}_{+} .
$$

We can now describe the mentioned series expansion along $(P, v)$, together with a domain of convergence. According to 14, Lemma 5.3 and Thm. 3.4, a function $f \in \mathcal{A}\left(\mathrm{X}_{+}: \tau\right)$ admits a converging series expansion of the form

$$
f(m a v)=\sum_{\xi \in E} a^{\xi} q_{\xi}(P, v \mid f, \log a, m),
$$

for $m \in \mathrm{X}_{P, v,+}$ and $a \in A_{P \mathrm{q}}^{+}\left(R_{P, v}(m)^{-1}\right)$. The set $E$ in (6.1) is a subset of $\mathfrak{a}_{P \mathrm{qC}}^{*}$ contained in a set of the form $E_{0}-\mathbb{N} \Delta_{r}(P):=E_{0}+\left(-\mathbb{N} \Delta_{r}(P)\right)$, with $E_{0} \subset \mathfrak{a}_{P \mathrm{qC}}^{*}$ finite. In addition, there exists a $k \in \mathbb{N}$ such that, for every $\xi \in E$, the expression $q_{\xi}(P, v \mid f)$ belongs to $P_{k}\left(\mathfrak{a}_{P \mathrm{q}}, C^{\infty}\left(\mathrm{X}_{P, v,+}: \tau_{P}\right)\right)$, the space of polynomial functions $\mathfrak{a}_{P \mathrm{q}} \rightarrow C^{\infty}\left(\mathrm{X}_{P, v,+}: \tau_{P}\right)$ of degree at most $k$. Here $\tau_{P}$ stands for $\left.\tau\right|_{K_{P}}$.

The series on the right-hand side of (6.1) converges neatly in the sense of 14], Def. 1.2, for each $m \in \mathrm{X}_{P, v,+}$, and for $a$ in the indicated range (depending on $m$ ). The functions $q_{\xi}$ are uniquely determined by these properties.

The set of $\xi \in E$ for which $q_{\xi}(P, v \mid f) \neq 0$ is called the set of exponents of $f$ along $(P, v)$, and denoted by $\operatorname{Exp}(P, v \mid f)$. We agree to write $q_{\xi}=0$ for $\xi \in \mathfrak{a}_{P \mathrm{qC}}^{*} \backslash E$.

Using the above terminology we shall introduce the space $\mathcal{E}_{Q}^{\text {hyp }}(\mathrm{X}: \tau)$ in a number of steps. First, following [14], Def. 12.1, we introduce a suitable space of meromorphic families of spherical functions. We agree to write $P_{0}$ for the standard minimal $\sigma$-parabolic subgroup. An index or superscript $P_{0}$ will be replaced by 0 . In particular, $\mathrm{X}_{0, v}=\mathrm{X}_{P_{0}, v}$ and $\tau_{0}=\tau_{P_{0}}$. Note that $\mathrm{X}_{0, v}=\mathrm{X}_{P, v}$ and $\tau_{0}=\tau_{P}$ for every $P \in \mathcal{P}_{\sigma}^{\min }$. 
Definition 6.1 Let $Q \in \mathcal{P}_{\sigma}$ and let $Y \subset{ }^{*} \mathfrak{a}_{Q \mathrm{qC}}^{*}$ be a finite subset. We define

$$
C_{Q, Y}^{\mathrm{ep}, \mathrm{hyp}}\left(\mathrm{X}_{+}: \tau\right)
$$

to be the space of functions $f: \mathfrak{a}_{Q \mathrm{qC}}^{*} \times \mathrm{X}_{+} \rightarrow V_{\tau}$, meromorphic in the first variable, for which there exist a constant $k \in \mathbb{N}$, a $\Sigma_{r}(Q)$-hyperplane configuration $\mathcal{H}$ in $\mathfrak{a}_{Q \mathrm{qC}}^{*}$ and a function $d: \mathcal{H} \rightarrow \mathbb{N}$ such that the following conditions are fulfilled.

(a) The function $\lambda \mapsto f_{\lambda}$ belongs to $\mathcal{M}\left(\mathfrak{a}_{Q \mathrm{qC}}^{*}, \mathcal{H}, d, C^{\infty}\left(\mathrm{X}_{+}: \tau\right)\right)$.

(b) For every $P \in \mathcal{P}_{\sigma}^{\min }$ and $v \in N_{K}\left(\mathfrak{a}_{\mathrm{q}}\right)$ there exist (necessarily unique) functions $q_{s, \xi}(P, v \mid f)$ in $P_{k}\left(\mathfrak{a}_{\mathrm{q}}\right) \otimes \mathcal{M}\left(\mathfrak{a}_{Q \mathrm{qc}}^{*}, \mathcal{H}, d, C^{\infty}\left(\mathrm{X}_{0, v}: \tau_{0}\right)\right)$, for $s \in$ $W / W_{Q}$ and $\xi \in-s W_{Q} Y+\mathbb{N} \Delta(P)$, with the following property. For all $\lambda \in \mathfrak{a}_{Q \mathrm{qC}}^{*} \backslash \cup \mathcal{H}, m \in \mathrm{X}_{0, v}$ and $a \in A_{\mathrm{q}}^{+}(P)$,

$$
f_{\lambda}(m a v)=\sum_{s \in W / W_{Q}} a^{s \lambda-\rho_{P}} \sum_{\xi \in-s W_{Q} Y+\mathbb{N} \Delta(P)} a^{-\xi} q_{s, \xi}(P, v \mid f, \log a)(\lambda, m),
$$

where the $\Delta(P)$-exponential polynomial series with coefficients in $V_{\tau}$ converges neatly on $A_{\mathrm{q}}^{+}(P)$.

(c) For every $P \in \mathcal{P}_{\sigma}^{\min }, v \in N_{K}\left(\mathfrak{a}_{\mathrm{q}}\right)$ and $s \in W / W_{Q}$, the series

$$
\sum_{\xi \in-s W_{Q} Y+\mathbb{N} \Delta(P)} a^{-\xi} q_{s, \xi}(P, v \mid f, \log a)
$$

converges neatly on $A_{\mathrm{q}}^{+}(P)$, as an exponential polynomial series with coefficients in the space $\mathcal{M}\left(\mathfrak{a}_{Q \mathrm{qC}}^{*}, \mathcal{H}, d, C^{\infty}\left(\mathrm{X}_{0, v}: \tau_{0}\right)\right)$.

Finally, we define

$$
C_{0}^{\text {ep,hyp }}\left(\mathrm{X}_{+}: \tau\right):=C_{P_{0},\{0\}}^{\mathrm{ep}, \mathrm{hyp}}\left(\mathrm{X}_{+}: \tau\right) .
$$

Remark 6.2 If $Q^{\prime} \in \mathcal{P}_{\sigma}$ and $\mathfrak{a}_{Q^{\prime} \mathrm{q}}=\mathfrak{a}_{Q \mathrm{q}}$, then $\Sigma_{r}(Q) \subset \Sigma_{r}\left(Q^{\prime}\right) \cup\left[-\Sigma_{r}\left(Q^{\prime}\right)\right]$. Hence, the notions of $\Sigma_{r}(Q)$ - and $\Sigma_{r}\left(Q^{\prime}\right)$-configuration coincide. It follows that the space (6.2) depends on $Q$ through its $\sigma$-split component $\mathfrak{a}_{Q \mathrm{q}}$.

It is sufficient to require conditions (b) and (c) either for all $P \in \mathcal{P}_{\sigma}^{\min }$ and a fixed $v$, or for a fixed $P \in \mathcal{P}_{\sigma}^{\min }$ and all $v$ in a choice of representatives for $W / W_{K \cap H}$ in $N_{K}\left(\mathfrak{a}_{\mathrm{q}}\right)$; see [14, Rem. 7.2, for details.

If $f \in C_{Q, Y}^{\mathrm{ep}, \mathrm{hyp}}\left(\mathrm{X}_{+}: \tau\right)$, then following [14], Def. 12.4, we define the asymptotic degree of $f$, denoted $\operatorname{deg}_{a}(f)$, to be the smallest integer $k$ for which there exist $\mathcal{H}, d$ such that the conditions of Definition 6.1 are fulfilled. Moreover, we denote by $\mathcal{H}_{f}$ the smallest $\Sigma_{r}(Q)$-configuration in $\mathfrak{a}_{Q \mathrm{qC}}^{*}$ such that the conditions of Definition 6.1 are fulfilled with $k=\operatorname{deg}_{a}(f)$ and for some $d: \mathcal{H}_{f} \rightarrow \mathbb{N}$. We denote by $d_{f}$ the $\preceq$-minimal map $\mathcal{H}_{f} \rightarrow \mathbb{N}$ for which the conditions of the definition are fulfilled with $\mathcal{H}=\mathcal{H}_{f}$ and $k=\operatorname{deg}_{a} f$. Finally, we put $\operatorname{reg}_{a}(f):=\mathfrak{a}_{Q \mathrm{qC}}^{*} \backslash \cup \mathcal{H}_{f}$.

We extend $\mathfrak{a}_{\mathrm{q}}$ to a Cartan subspace $\mathfrak{b}$ of $\mathfrak{q}$; clearly, $\mathfrak{b}$ is $\theta$-stable. If $\mu \in \mathfrak{b}_{\mathbb{C}}^{*}$, then $I_{\mu}:=\operatorname{ker} \gamma(\cdot: \mu)$ is an ideal of codimension one in $\mathbb{D}(\mathrm{X})$. 
If $P \in \mathcal{P}_{\sigma}$, we write $\mathrm{D}_{P}$ for the set of finitely supported maps $\delta:{ }^{*} \mathfrak{b}_{P \mathbb{C}}^{*} \rightarrow \mathbb{N}$, see (2.6). For $\delta \in \mathrm{D}_{P}$ and $\lambda \in \mathfrak{a}_{P \mathrm{qC}}^{*}$ we define the ideal $I_{\delta, \lambda}$ in $\mathbb{D}(\mathrm{X})$ by

$$
I_{\delta, \lambda}:=\prod_{\Lambda \in \operatorname{supp} \delta} I_{\Lambda+\lambda}^{\delta(\Lambda)} .
$$

This ideal is cofinite, since it is a product of finitely generated cofinite ideals.

Following [14, Def. 12.8, we introduce the following space of meromorphic families of $\mathbb{D}(\mathrm{X})$-finite functions.

Definition 6.3 Let $Q \in \mathcal{P}_{\sigma}$ and $\delta \in \mathrm{D}_{Q}$. Then for $Y \subset{ }^{*} \mathfrak{a}_{Q \mathrm{qC}}^{*}$ a finite subset we define

$$
\mathcal{E}_{Q, Y}^{\text {hyp }}\left(\mathrm{X}_{+}: \tau: \delta\right)
$$

to be the space of functions $f \in C_{Q, Y}^{\mathrm{ep}, \text { hyp }}\left(\mathrm{X}_{+}: \tau\right)$ such that, for all $\lambda \in \mathfrak{a}_{Q \mathrm{qc}}^{*} \backslash \mathcal{H}_{f}$, the function $f_{\lambda}: x \mapsto f(\lambda, x)$ is annihilated by the cofinite ideal $I_{\delta, \lambda}$.

Finally, we define

$$
\mathcal{E}_{0}^{\text {hyp }}\left(\mathrm{X}_{+}: \tau: \delta\right):=\mathcal{E}_{P_{0},\{0\}}^{\text {hyp }}\left(\mathrm{X}_{+}: \tau: \delta\right) .
$$

Following [14, Def. 12.8, we introduce the following subspace of meromorphic families of $\mathbb{D}(\mathrm{X})$-finite functions in $C^{\infty}\left(\mathrm{X}_{+}: \tau\right)$ satisfying a certain additional assumption. Let $\mathcal{P}_{\sigma}^{1}$ be the collection of parabolic subgroups $P \in \mathcal{P}_{\sigma}$ whose $\sigma$-split component $\mathfrak{a}_{P \mathrm{q}}$ has codimension one in $\mathfrak{a}_{\mathrm{q}}$.

Definition 6.4 Let $Q \in \mathcal{P}_{\sigma}$. Then for $\delta \in \mathrm{D}_{Q}$ and $Y \subset{ }^{*} \mathfrak{a}_{Q \mathrm{qC}}^{*}$ a finite subset we define $\mathcal{E}_{Q, Y}^{\text {hyp }}\left(\mathrm{X}_{+}: \tau: \delta\right)_{\text {glob }}$ to be the space of families $f \in \mathcal{E}_{Q, Y}^{\text {hyp }}\left(\mathrm{X}_{+}: \tau: \delta\right)$ satisfying the following condition.

For every $s \in W$, every $P \in \mathcal{P}_{\sigma}^{1}$ with $s \mathfrak{a}_{Q \mathrm{q}} \not \subset \mathfrak{a}_{P \mathrm{q}}$ and all $v \in N_{K}\left(\mathfrak{a}_{\mathrm{q}}\right)$, there exists an open dense subset $\Omega$ of $\operatorname{reg}_{a} f$ with the following property. For every $\lambda \in \Omega$, every $\left.\xi \in s \lambda\right|_{\mathfrak{a}_{P q}}+\left.W_{P} s W_{Q} Y\right|_{\mathfrak{a}_{P q}}-\rho_{P}-\mathbb{N} \Delta_{r}(P)$ and all $X \in \mathfrak{a}_{P \mathrm{q}}$, the function $m \mapsto q_{\xi}\left(P, v \mid f_{\lambda}, X, m\right)$, originally defined on $\mathrm{X}_{P, v,+}$, extends smoothly to all of $\mathrm{X}_{P, v}$.

Remark 6.5 In [14, Def. 9.5 and Def. 8.4, a family $f$ satisfying the property stated in the above display was said to be $[s]$-global along $(P, v)$.

Definition 6.6 Let $Q \in \mathcal{P}_{\sigma}$. For $\delta \in \mathrm{D}_{Q}$ and $Y \subset{ }^{*} \mathfrak{a}_{Q \mathrm{q}}^{*}$ a finite subset, we define $\mathcal{E}_{Q, Y}^{\text {hyp }}(\mathrm{X}: \tau: \delta)$ to be the space of families $f \in \mathcal{E}_{Q, Y}^{\text {hyp }}\left(\mathrm{X}_{+}: \tau: \delta\right)_{\text {glob }}$ satisfying the following condition.

For all $\nu$ in the complement of a locally finite union of analytic null-sets, the function $f_{\nu}$, initially defined on $\mathrm{X}_{+}$, extends to a smooth function on all of X.

Finally, we define

$$
\mathcal{E}_{Q, Y}^{\text {hyp }}(\mathrm{X}: \tau):=\cup_{\delta} \mathcal{E}_{Q, Y}^{\text {hyp }}(\mathrm{X}: \tau: \delta) \quad \text { and } \quad \mathcal{E}_{Q}^{\text {hyp }}(\mathrm{X}: \tau):=\cup_{Y} \mathcal{E}_{Q, Y}^{\text {hyp }}(\mathrm{X}: \tau),
$$

where $\delta$ runs over $\mathrm{D}_{Q}$ and $Y$ over the collection of finite subsets of ${ }^{*} \mathfrak{a}_{Q \mathrm{q}}^{*}$. 
Remark 6.7 One readily verifies that the space $\mathcal{E}_{Q, Y}^{\text {hyp }}(\mathrm{X}: \tau: \delta)$ depends on $Q$ through its split component $\mathfrak{a}_{Q \mathrm{q}}$. See also Remark 6.2

Remark 6.8 If $G$ has compact center modulo $H$, then $\mathfrak{a}_{G q}=\{0\}$. Therefore, the map $f \mapsto f_{0}$ defines an embedding of $\mathcal{E}_{G}^{\text {hyp }}(\mathrm{X}: \delta)$ into $\mathcal{A}(\mathrm{X}: \tau)$, the space of $\mathbb{D}(\mathrm{X})$-finite functions in $C^{\infty}(\mathrm{X}: \tau)$. We claim that this map is in fact a bijection. To see this, let $g \in \mathcal{A}(\mathrm{X}: \tau)$. Then by []. Thm. 5.3, the family $f: \mathfrak{a}_{G \mathrm{qC}}^{*} \times \mathrm{X} \rightarrow V_{\tau}$ defined by $f_{0}=g$ belongs to $C_{G, Y}^{\text {ep, hyp }}\left(\mathrm{X}_{+}: \tau\right)$ for some finite subset $Y \subset{ }^{*} \mathfrak{a}_{G \mathrm{qC}}^{*}=$ $\mathfrak{a}_{\mathrm{qC}}^{*}$. Moreover, $\mathbb{D}(\mathrm{X}) g$ is a finite dimensional space. In the notation introduced before Definition 6.3 let $S$ be the set of $\Lambda \in{ }^{*} \mathfrak{b}_{G \mathbb{C}}^{*}=\mathfrak{b}_{\mathbb{C}}^{*}$ such that $\mathbb{D}(\mathrm{X}) g$ has a non-trivial subspace on which $\mathbb{D}(\mathrm{X})$ acts by the character $\gamma(\cdot: \Lambda)$. Then there exists a map $\delta:{ }^{*} \mathfrak{b}_{P \mathbb{C}}^{*} \rightarrow \mathbb{N}$ supported by $S$, such that $g$ is annihilated by $I_{\delta, 0}$. It is now readily seen that the family $f$ belongs to $\mathcal{E}_{G, Y}^{\text {hyp }}(\mathrm{X}: \tau: \delta)$.

Lemma 6.9 Let $f \in \mathcal{E}_{Q}^{\text {hyp }}(\mathrm{X}: \tau)$ and put $\mathcal{H}=\mathcal{H}_{f}$ and $d=d_{f}$. Then $\nu \mapsto f_{\nu}$ is a meromorphic $C^{\infty}(\mathrm{X}: \tau)$-valued function in the space $\mathcal{M}\left(\mathfrak{a}_{Q \mathrm{qC}}^{*}, \mathcal{H}, d, C^{\infty}(\mathrm{X}: \tau)\right)$.

Proof: This follows by using condition (a) of Definition 6.1 and applying Cor. 18.2 of [14].

Remark 6.10 Let $\delta \in \mathrm{D}_{Q}$ and let $Y \subset{ }^{*} \mathfrak{a}_{Q \mathrm{qC}}^{*}$ be a finite subset. It can be shown that every family $f \in \mathcal{E}_{Q, Y}^{\text {hyp }}\left(\mathrm{X}_{+}: \tau: \delta\right)$ that satisfies the displayed condition of Definition [6.6] automatically belongs to $\mathcal{E}_{Q, Y}^{\text {hyp }}\left(\mathrm{X}_{+}: \tau: \delta\right)_{\text {glob }}$, hence to $\mathcal{E}_{O}^{\text {hyp }}(\mathrm{X}: \tau)$. In case $\max \delta \leq 1$, this follows from [5], Thm. 12.8. For general $\delta \in \mathrm{D}_{Q}$ one may proceed along similar lines, see also [42], Chapter 4. However, we shall not need such a result in the present paper, since in all cases where we could apply it, the property of Definition 6.4 has already been established in 14 for the functions involved. The present remark justifies the notation used.

The following special case of the vanishing theorem of [14] will play an important role in the rest of this paper.

Theorem 6.11 (Vanishing theorem) Let $Q \in \mathcal{P}_{\sigma}$ be standard and let ${ }^{Q} \mathcal{W} \subset$ $N_{K}\left(\mathfrak{a}_{\mathrm{q}}\right)$ be a choice of representatives for $W_{Q} \backslash W / W_{K \cap H}$. Let $f \in \mathcal{E}_{Q}^{\text {hyp }}(\mathrm{X}: \tau)$ and assume that there exists a non-empty open subset $\Omega \subset \operatorname{reg}_{a} f$ such that, for each $v \in{ }^{Q} \mathcal{W}$,

$$
q_{\nu-\rho_{P}}\left(P, v \mid f_{\nu}\right)=0, \quad(\nu \in \Omega) .
$$

Then $f=0$.

Proof: This is a special case of [14], Thm. 12.10.

We shall often use the vanishing theorem in combination with the following lemma to relate families of eigenfunctions.

Lemma 6.12 Let $P, Q \in \mathcal{P}_{\sigma}$, let $s \in W$ be such that $s \mathfrak{a}_{P \mathrm{q}}=\mathfrak{a}_{Q \mathrm{q}}$ and assume that $f \in \mathcal{E}_{P, Y}^{\mathrm{hyp}}(\mathrm{X}: \tau)$, with $Y \subset{ }^{*} \mathfrak{a}_{P \mathrm{qC}}^{*}$ a finite subset. Then the family $f^{s}: \mathfrak{a}_{Q \mathrm{qC}}^{*} \times$ $\mathrm{X} \rightarrow V_{\tau}$, defined by $f^{s}(\nu, x)=f\left(s^{-1} \nu, x\right)$, belongs to $\mathcal{E}_{Q, s Y}^{\text {hyp }}(\mathrm{X}: \tau)$. 
Proof: In view of Remark 6.7 we may assume that $Q=s P s^{-1}$. There exists a $\delta \in \mathrm{D}_{P}$ such that $f \in \mathcal{E}_{P, Y}^{\mathrm{hyp}}(\mathrm{X}: \tau: \delta)$. By Lemma 3.7 there exists a $\tilde{s} \in W(\mathfrak{b})$ such that $s=\left.\tilde{s}\right|_{\mathfrak{a}_{\mathrm{q}}}$. The element $\tilde{s}$ maps ${ }^{*} \mathfrak{b}_{P \mathrm{C}}$ onto ${ }^{*} \mathfrak{b}_{Q \mathrm{C}}$. Let $\tilde{s}^{*}$ denote its transpose ${ }^{*} \mathfrak{b}_{Q \mathbb{C}}^{*} \rightarrow{ }^{*} \mathfrak{b}_{P \mathbb{C}}^{*}$. Then $\delta \circ \tilde{s}^{*} \in \mathrm{D}_{Q}$. Moreover, one readily checks from the definitions that $f^{s} \in \mathcal{E}_{Q, s Y}^{\text {hyp }}\left(\mathrm{X}: \tau: \delta \circ \tilde{s}^{*}\right)$.

\section{Meromorphy of asymptotic expansions}

Let $f$ belong to the space $\mathcal{E}_{Q}^{\text {hyp }}(\mathrm{X}: \tau)$, defined in Definition 6.6 The mentioned definition refers back to Definition 6.1 according to which, for $v \in N_{K}\left(\mathfrak{a}_{\mathrm{q}}\right)$ and for $P$ a minimal group in $\mathcal{P}_{\sigma}$, the function $f_{\nu}$ admits an expansion along $(P, v)$ that depends meromorphically on the parameter $\nu \in \mathfrak{a}_{Q \mathrm{qc}}^{*}$ in a well defined sense. It follows from [14 that an analogous result holds for arbitrary $P \in \mathcal{P}_{\sigma}$. For its formulation, we need a particular type of subset of the symmetric space $\mathrm{X}_{P, v}$. For $1 \leq R \leq \infty$, we define the set

$$
\mathrm{X}_{P, v,+}[R]:=\left\{x \in \mathrm{X}_{P, v,+} \mid R_{P, v}(x)<R\right\} ;
$$

see [14, Eqn. (3.7) for details.

We also need an equivalence relation $\sim_{P \mid Q}$ on $W$ to describe asymptotic exponents along $(P, v)$ without redundance. The relation is defined by

$$
s \sim_{P \mid Q} t \Longleftrightarrow \forall \nu \in \mathfrak{a}_{Q \mathrm{q}}^{*}:\left.s \nu\right|_{\mathfrak{a}_{P \mathrm{q}}}=\left.t \nu\right|_{\mathfrak{a}_{P \mathrm{q}}} .
$$

If $Y \subset{ }^{*} \mathfrak{a}_{Q \mathrm{qC}}^{*}$ is a finite subset and $\sigma \in W / \sim_{P \mid Q}$, we put

$$
\sigma \cdot Y:=\left\{\left.s \eta\right|_{\mathfrak{a}_{P q}} \mid s \in \sigma, \eta \in Y\right\}
$$

see 14], $\S 6$, for details. We recall from [14, Lemma 6.5, that $W / \sim_{P \mid Q} \simeq W / W_{Q}$, if $P \in \mathcal{P}_{\sigma}^{\min }$ and $Q \in \mathcal{P}_{\sigma}$.

Proposition 7.1 Let $Q \in \mathcal{P}_{\sigma}, \delta \in \mathrm{D}_{Q}$ and $Y \subset{ }^{*} \mathfrak{a}_{Q \mathrm{q}}^{*}$ a finite subset. Let $f$ be a family in $C_{Q, Y}^{\mathrm{ep}, \mathrm{hyp}}\left(\mathrm{X}_{+}: \tau\right)$ and put $k=\operatorname{deg}_{a} f$.

Let $P \in \mathcal{P}_{\sigma}$ and $v \in N_{K}\left(\mathfrak{a}_{\mathrm{q}}\right)$. Then $\left.\operatorname{Exp}\left(P, v \mid f_{\nu}\right) \subset W(\nu+Y)\right|_{\mathfrak{a}_{P \mathrm{q}}}-\rho_{P}-$ $\mathbb{N} \Delta_{r}(P)$, for every $\nu \in \operatorname{reg}_{a} f$. Moreover, there exist unique functions

$$
q_{\sigma, \xi}(P, v \mid f) \in P_{k}\left(\mathfrak{a}_{P \mathrm{q}}\right) \otimes \mathcal{M}\left(\mathfrak{a}_{Q \mathrm{qC}}^{*}, \mathcal{H}_{f}, d_{f}, C^{\infty}\left(\mathrm{X}_{P, v,+}: \tau_{P}\right)\right),
$$

for $\sigma \in W / \sim_{P \mid Q}$ and $\xi \in-\sigma \cdot Y+\mathbb{N} \Delta_{r}(P)$, such that, for every $\nu \in \operatorname{reg}_{a} f$,

$$
f_{\nu}(\text { mav })=\sum_{\sigma \in W / \sim_{P \mid Q}} a^{\sigma \nu-\rho_{P}} \sum_{\xi \in-\sigma \cdot Y+\mathbb{N} \Delta_{r}(P)} a^{-\xi} q_{\xi}(P, v \mid f, \log a)(\nu, m),
$$

for all $m \in \mathrm{X}_{P, v,+}$ and $a \in A_{P q}^{+}\left(R_{P, v}(m)^{-1}\right)$, where the exponential polynomial series in the variable a with coefficients in $V_{\tau}$ is neatly convergent in the indicated range. In particular, for all $\nu$ in an open dense subset of $\mathfrak{a}_{Q \mathrm{qC}}^{*}$ and all $\sigma \in W / \sim_{P \mid Q}$ and $\xi \in-\sigma \cdot Y+\mathbb{N} \Delta_{r}(P)$

$$
q_{\sigma, \xi}(P, v \mid f)(X, \nu)=q_{\left.\sigma \nu\right|_{\mathfrak{a}_{P q}}-\rho_{P}-\xi}\left(P, v \mid f_{\nu}, X\right), \quad\left(X \in \mathfrak{a}_{P \mathrm{q}}\right) .
$$


Finally, for each $\sigma \in W / \sim_{P \mid Q}$ and every $R>1$, the series

$$
\sum_{\xi \in-\sigma \cdot Y+\mathbb{N} \Delta_{r}(P)} a^{-\xi} q_{\sigma, \xi}(P, v \mid f, \log a)
$$

converges neatly on $A_{P \mathrm{q}}^{+}\left(R^{-1}\right)$ as a $\Delta_{r}(P)$-exponential polynomial series with coefficients in $\mathcal{M}\left(\mathfrak{a}_{Q \mathrm{qc}}^{*}, C^{\infty}\left(\mathrm{X}_{P, v,+}[R]: \tau_{P}\right)\right.$.

Proof: This follows from [14, Thm. 7.7 and Lemma 12.7.

The following result is based on the meromorphic nature of the series in the above proposition. It may be considered a natural companion to [14], Lemma 7.9 .

Lemma 7.2 Let $Q \in \mathcal{P}_{\sigma}, Y \subset{ }^{*} \mathfrak{a}_{Q \mathrm{qc}}^{*}$ a finite subset and $f \in C_{Q, Y}^{\mathrm{ep}, \mathrm{hyp}}\left(\mathrm{X}_{+}: \tau\right)$. Let $P \in \mathcal{P}_{\sigma}, v \in N_{K}\left(\mathfrak{a}_{\mathrm{q}}\right)$. Assume that for every $\sigma \in W / \sim_{P \mid Q}$ a set $E_{\sigma} \subset$ $-\sigma \cdot Y+\mathbb{N} \Delta_{r}(P)$ is given such that, for $\nu$ in a non-empty open subset $\Omega$ of $\operatorname{reg}_{a} f$

$$
\left.\operatorname{Exp}\left(P, v \mid f_{\nu}\right) \subset \bigcup_{\sigma \in W / \sim_{P \mid Q}} \sigma \nu\right|_{\mathfrak{a}_{P \mathfrak{q}}}-\rho_{P}-E_{\sigma} .
$$

Then (7.3) holds for every $\nu \in \operatorname{reg} f$.

Proof: Let $\sigma_{0} \in W / \sim_{P \mid Q}$ and $\xi \in-\sigma_{0} \cdot Y+\mathbb{N} \Delta_{r}(P)$ be such that $q_{\sigma_{0}, \xi}(P, v \mid$ $f) \neq 0$. By Proposition [7.1] there exists an open dense subset $\Omega \subset \operatorname{reg}_{a} f$ such that, for all $\nu \in \Omega$, (7.2) is valid. In particular, it follows that, for $\nu \in \Omega$, $\left.\sigma_{0} \nu\right|_{\mathfrak{a}_{P \mathrm{q}}}-\rho_{P}-\xi \in \operatorname{Exp}\left(P, v \mid f_{\nu}\right)$; hence, $\left.\sigma_{0} \nu\right|_{\mathfrak{a}_{P \mathrm{q}}}-\rho_{P}-\xi$ belongs to the union on the right-hand side of (17.3). By [14], Lemma 6.2, the sets $\left.\sigma \nu\right|_{\mathfrak{a}_{P q}}+\sigma \cdot Y-\mathbb{N} \Delta_{r}(P)$, for $\sigma \in W / \sim_{P \mid Q}$, are mutually disjoint, for $\nu$ in an open dense subset $\Omega^{\prime}$ of $\Omega$. It follows that $\xi \in E_{\sigma_{0}}$.

From the above and Proposition [7.1 we conclude that for all $\sigma \in W / \sim_{P \mid Q}$ and $\xi \in\left(-\sigma \cdot Y+\mathbb{N} \Delta_{r}(P)\right) \backslash E_{\sigma}$, the meromorphic function $\nu \mapsto q_{\sigma, \xi}(P, v \mid f, \nu)$ is zero. Hence, for $\nu \in \operatorname{reg}_{a} f$,

$$
f_{\nu}(m a v)=\sum_{\sigma \in W / \sim_{P \mid Q}} a^{\sigma \nu-\rho_{P}} \sum_{\xi \in E_{\sigma}} a^{-\xi} q_{\sigma, \xi}(P, v \mid f)(\log a, \nu, m),
$$

for $m \in \mathrm{X}_{P, v,+}$ and $a \in A_{P \mathrm{q}}^{+}\left(R_{P, v}(m)^{-1}\right)$. Thus, the inclusion (7.3) holds for $\nu \in \operatorname{reg}_{a} f$. It remains to extend the domain of its validity to all of reg $f$.

Let $R \geq 1$. Then for each $\sigma \in W / \sim_{P \mid Q}$, the series

$$
F_{\sigma}(a)=\sum_{\xi \in E_{\sigma}} a^{-\xi} q_{\sigma, \xi}(P, v \mid f)(\log a)
$$

converges as a $\Delta_{r}(P)$-exponential polynomial series in $a \in A_{P \mathrm{q}}^{+}\left(R^{-1}\right)$, with coefficients in the space $\mathcal{M}\left(\mathfrak{a}_{Q \mathrm{qC}}^{*}, \mathcal{H}, d, C^{\infty}\left(\mathrm{X}_{P, v,+}[R]: \tau_{P}\right)\right)$; here $\mathcal{H}=\mathcal{H}_{f}$ and $d=d_{f}$. 
Let $\nu_{0} \in \mathfrak{a}_{Q \mathrm{qC}}^{*}$ be such that the meromorphic $C^{\infty}\left(\mathrm{X}_{+}: \tau\right)$-valued function $\nu \mapsto f_{\nu}$ is regular at $\nu_{0}$. Let $\mathcal{H}\left(\nu_{0}\right)$ be the collection of $H \in \mathcal{H}$ that contain $\nu_{0}$. Since $\mathcal{H}$ is a $\Sigma_{r}(Q)$-configuration in $\mathfrak{a}_{Q \mathrm{qC}}^{*}$, the collection $\mathcal{H}\left(\nu_{0}\right)$ is finite and there exists a bounded open neighborhood $\omega$ of $\nu_{0}$ in $\operatorname{reg}(f)$ such that $\mathcal{H}\left(\nu_{0}\right)=\{H \in \mathcal{H} \mid H \cap \omega \neq \emptyset\}$. Put $\pi=\pi_{\omega, d}$, see (4.3). Then $\left.\varphi \mapsto \pi \varphi\right|_{\omega}$ defines a continuous linear map from $\mathcal{M}\left(\mathfrak{a}_{Q \mathrm{qC}}^{*}, \mathcal{H}, d, C^{\infty}\left(\mathrm{X}_{P, v,+}[P]: \tau_{P}\right)\right)$ into $\mathcal{O}\left(\mathfrak{a}_{Q \mathrm{qc}}^{*}, C^{\infty}\left(\mathrm{X}_{P, v,+}[R]: \tau_{P}\right)\right)$. In particular, the series (17.5) multiplied with $\pi$ converges neatly on $A_{P \mathrm{q}}^{+}\left(R^{-1}\right)$ as an exponential polynomial series with values in the space $\mathcal{O}\left(\omega, C^{\infty}\left(\mathrm{X}_{P, v,+}[R]: \tau_{P}\right)\right)$.

It follows from [14], Lemma 10.5, that there exists a $u \in S\left(\mathfrak{a}_{Q \mathrm{q}}^{*}\right)$, such that $\varphi\left(\nu_{0}\right)=u(\pi \varphi)\left(\nu_{0}\right)$, for $\varphi \in \mathcal{O}(\omega)$. We apply this to $\nu \mapsto f_{\nu}$. Then,

$$
\begin{aligned}
f\left(\nu_{0}, \text { mav }\right) & =u[\pi(\cdot) f(\cdot, \text { mav })]\left(\nu_{0}\right) \\
& =\sum_{\sigma \in W / \sim_{P \mid Q}} a^{\sigma \nu_{0}-\rho_{P}} \sum_{j=1}^{k} p_{\sigma, j}(\log a) U_{\sigma, j}\left[\pi(\cdot) F_{\sigma}(a)(\cdot, m)\right]\left(\nu_{0}\right),
\end{aligned}
$$

with finitely many $p_{\sigma, j} \in P\left(\mathfrak{a}_{P \mathrm{q}}\right)$ and $U_{\sigma, j} \in S\left(\mathfrak{a}_{Q \mathrm{q}}^{*}\right)$, as is readily seen by application of the Leibniz rule. Note that $\operatorname{deg} p_{\sigma, j}+\operatorname{order}\left(U_{\sigma, j}\right) \leq l:=\operatorname{order}(u)$.

From [14, Lemma 1.10, we obtain that the elements $U_{\sigma, j} \in S\left(\mathfrak{a}_{Q q}^{*}\right)$, viewed as constant coefficient differential operators in the variable $\nu$, may be applied termwise to the series for $\pi(\cdot) F_{\sigma}(a)(\cdot, m)$, without disturbing the nature of the convergence of the series. This leads to the existence of polynomial functions $\bar{q}_{\sigma, \xi} \in P_{k+l}\left(\mathfrak{a}_{P \mathrm{q}}\right) \otimes C^{\infty}\left(\mathrm{X}_{P, v,+}: \tau_{P}\right)$, for $\sigma \in W / \sim_{P \mid Q}, \xi \in E_{\sigma}$, such that

$$
f_{\nu_{0}}(m a v)=\sum_{\sigma \in W / \sim_{P \mid Q}} a^{\sigma \nu_{0}-\rho_{P}} \sum_{\xi \in E_{\sigma}} a^{-\xi} \bar{q}_{\sigma, \xi}(\log a, m),
$$

for $a \in A_{P \mathrm{q}}^{+}\left(R^{-1}\right)$ and $m \in \mathrm{X}_{P, v,+}[R]$. The series on the right-hand side converges neatly as a $\Delta_{r}(P)$-exponential polynomial series on $A_{P q}^{+}\left(R^{-1}\right)$, with coefficients in $C^{\infty}\left(\mathrm{X}_{P, v,+}[R]: \tau_{P}\right)$. It follows that the inclusion (7.3) is valid for $\nu=\nu_{0}$.

\section{Fourier inversion}

In this section we recall the Fourier inversion formula from 12 that will be the starting point for the derivation of the spherical Plancherel formula.

Let $\mathcal{W} \subset N_{K}\left(\mathfrak{a}_{\mathrm{q}}\right)$ be a choice of representatives for $W / W_{K \cap H}$. This choice determines the space ${ }^{\circ} \mathrm{C}={ }^{\circ} \mathrm{C}(\tau)$, defined as the formal Hilbert direct sum of finite dimensional Hilbert spaces

$$
{ }^{\circ} \mathcal{C}:=\bigoplus_{v \in \mathcal{W}} C^{\infty}\left(M / M \cap v H v^{-1}: \tau_{0}\right)
$$

where $\tau_{0}$ denotes the restriction of $\tau$ to $K_{0}:=K \cap M$. Given $P \in \mathcal{P}_{\sigma}^{\min }$, and $\psi \in{ }^{\circ} \mathcal{C}$, we define the normalized Eisenstein integral $E^{\circ}(P: \psi)$ as in $\underline{9}$, $\S 5$. This 
Eisenstein integral is a meromorphic $C^{\infty}(\mathrm{X}: \tau)$-valued function on $\mathfrak{a}_{\mathrm{qc}}^{*}$, which depends linearly on $\psi$. It naturally arises in representation theory, essentially as a sum of matrix coefficients of the minimal principal series of X. However, it can also be characterized by some of its analytic properties, as follows.

Proposition 8.1 Let $P \in \mathcal{P}_{\sigma}^{\min }$ and $\psi \in{ }^{\circ} \mathcal{C}$. The function $\nu \mapsto E^{\circ}(P: \psi: \nu)$ is the unique family in $\mathcal{E}_{P}^{\text {hyp }}(\mathrm{X}: \tau)$ with the following property. For each $v \in \mathcal{W}$ and for $\nu$ in a dense open subset of $\mathfrak{a}_{\mathrm{qC}}^{*}$,

$$
q_{\nu-\rho_{P}}\left(P, v \mid E^{\circ}(P: \psi: \nu), \cdot, m\right)=\psi_{v}(m) .
$$

Here $q_{\nu-\rho_{P}}$ is the coefficient in the expansion [6.1) for $f=E^{\circ}(P: \psi: \nu)$.

Remark 8.2 It is implicit in the above formulation that the function on the left-hand side of the above equation is constant as a polynomial function of the variable indicated by the dot. It is known that for $\nu$ in a dense open subset of $\mathfrak{a}_{\mathrm{qC}}^{*}$, there are no terms with $\log a$ in the expansion (6.1) with $f=E^{\circ}(P: \psi: \nu)$; see [10, Thm. 7.5.

Proof: Uniqueness follows from the vanishing theorem, Thm. 6.11 Thus, it suffices to show that, for $\psi \in{ }^{\circ} \mathcal{C}$, the $C^{\infty}(\mathrm{X}: \tau)$-valued meromorphic function $f: \nu \mapsto E^{\circ}(\psi: \nu)$ on $\mathfrak{a}_{\mathrm{qC}}^{*}$ satisfies the properties mentioned above. By linearity with respect to $\psi$ we may assume that $\psi \in{ }^{\circ} \mathcal{C}[\Lambda]$, for some $\Lambda \in i \mathfrak{b}_{\mathrm{k}}^{*}=i^{*} \mathfrak{b}_{P}^{*}$, in the notation of [14, text preceding Lemma 14.3. It now follows from [14, Lemma 14.5 , that $f \in \mathcal{E}_{P}^{\text {hyp }}\left(\mathrm{X}_{+}: \tau: \delta_{\Lambda}\right)_{\text {glob }}$, where $\delta_{\Lambda}$ is the characteristic function of the subset $\{\Lambda\}$ of $*^{*} \mathfrak{b}_{P \mathbb{C}}^{*}$. Finally, it follows from [9, beginning of Sect. 5 , that $\nu \mapsto f_{\nu}$ is a $C^{\infty}(\mathrm{X}: \tau)$-valued meromorphic function on $\mathfrak{a}_{\mathrm{qC}}^{*}$. Therefore, $f \in \mathcal{E}_{P}^{\mathrm{hyp}}(\mathrm{X}: \tau)$.

Combining [9], Eqns. (49), (45) and the display following the latter equation, we finally obtain that

$$
q_{\nu-\rho_{P}}\left(P, v \mid f_{\nu}: \cdot: m\right)=\left[p_{P \mid P, 0}(1: \nu) C_{P \mid P}(1: \nu)^{-1} \psi\right]_{v}(m)=\psi_{v}(m),
$$

for each $v \in \mathcal{W}$ and all $m \in M$.

Lemma 8.3 Let $P \in \mathcal{P}_{\sigma}^{\min }$ and $\psi \in{ }^{\circ} \mathcal{C}$. Let $f$ be the family in $\mathcal{E}_{P}^{\text {hyp }}(\mathrm{X}: \tau)$ defined by $f_{\nu}=E^{\circ}(P: \psi: \nu)$, for $\nu \in \mathfrak{a}_{\mathrm{qC}}^{*}$. Then the $\Sigma(P)$-configuration $\mathcal{H}_{f}$, defined as in the text preceding Definition 6.3 is real.

Proof: In view of 14, Eqn. (14.12), it suffices to prove a similar statement for the function $f$ of [14, Lemma 14.3. We observe that in the proof of the mentioned lemma, $\mathcal{H}_{f}$ is shown to be contained in a $\Sigma(P)$-configuration $\mathcal{H}^{\prime \prime}$. This configuration is given as $\mathcal{H}^{\prime \prime}=t^{-1} \mathcal{H} \cup \mathcal{H}^{\prime}$ with $t \in W$. Now $\mathcal{H}$ is real by 14], Cor. 14.2. It therefore remains to show that $\mathcal{H}^{\prime}$ is real. For this it suffices to show that the singular locus of the normalized $C$-function $C_{P \mid P}^{\circ}(t: \cdot)$ is the union of a real $\Sigma$-configuration. By [14, Eqn. (14.6), it suffices to show that the singular loci of $C_{P \mid P}(1: \cdot)^{-1}$ and $C_{P \mid P}(t: \cdot)$ are likewise. In view of [5], Cor. 15.5, it suffices to show that the functions $C_{Q \mid P}(1: \cdot)^{ \pm 1}$, for $Q \in \mathcal{P}_{\sigma}^{\min }$, all have a singular locus equal to the union of a real $\Sigma$-configuration. The latter assertion follows by the argument following the proof of Lemma 3.2 in [12]. 
We write $E^{\circ}(P: \cdot)$ for the meromorphic $C^{\infty}\left(\mathrm{X}, \operatorname{Hom}\left({ }^{\circ} \mathcal{C}, V_{\tau}\right)\right)$-valued function on $\mathfrak{a}_{\mathrm{qC}}^{*}$ given by

$$
E^{\circ}(P: \nu: x) \psi=E^{\circ}(P: \psi: \nu: x)
$$

for $x \in \mathrm{X}, \psi \in{ }^{\circ} \mathcal{C}$ and generic $\nu \in \mathfrak{a}_{\mathrm{qC}}^{*}$. Following [12], Eqn. (2.3), we define the dualized Eisenstein integral as the $C^{\infty}\left(\mathrm{X}, \operatorname{Hom}\left(V_{\tau},{ }^{\circ} \mathcal{C}\right)\right)$-valued meromorphic function on $\mathfrak{a}_{\mathrm{qc}}^{*}$ given by

$$
E^{*}(P: \nu: x):=E^{\circ}(P:-\bar{\nu}: x)^{*} .
$$

Finally, we introduce the partial Eisenstein integrals $E_{+, s}(P: \cdot)$, for $s \in W$, as in 12, Eqn. (2.9), see also [14, Eqn. (14.11). Let $1 \otimes \tau$ denote the natural representation of $K$ in $\operatorname{Hom}\left({ }^{\circ} \mathcal{C}, V_{\tau}\right) \simeq{ }^{\circ} \mathcal{C}^{*} \otimes V_{\tau}$. Then the partial Eisenstein integrals are meromorphic $C^{\infty}\left(\mathrm{X}_{+}: 1 \otimes \tau\right)$-valued functions on $\mathfrak{a}_{\mathrm{qC}}^{*}$. Moreover, for $\nu \in \mathfrak{a}_{\mathrm{qC}}^{*}$ a regular point, the partial Eisenstein integral $E_{+, s}(P: \nu)$ is $\mathbb{D}(\mathrm{X})$ finite. By 14, Lemma 5.3, it therefore has converging expansions like (6.1), but with $q_{\xi}\left(Q, v \mid E_{+, s}(P: \nu)\right)$ a $C^{\infty}\left(\mathrm{X}_{Q, v,+}: 1 \otimes \tau\right)$-valued polynomial function on $\mathfrak{a}_{Q q}$; see [14], Def. 2.1, for details. The exponents of the partial Eisenstein integrals are restricted by

$$
\operatorname{Exp}\left(P, v \mid E_{+, s}(P: \nu)\right) \subset s \lambda-\rho_{P}-\mathbb{N} \Delta(P),
$$

for all $v \in \mathcal{W}$. Finally, according to [14], Eqn. (165),

$$
E^{\circ}(P: \nu)=\sum_{s \in W} E_{+, s}(P: \nu) \quad \text { on } \quad \mathrm{X}_{+} .
$$

The mentioned properties determine the partial Eisenstein integrals completely, see [14, Lemma 2.2.

We shall now investigate the dependence of the Eisenstein integrals on the choice of $\mathcal{W}$. To this purpose, let ' $\mathcal{W}$ be a second choice of representatives for $W / W_{K \cap H}$ in $N_{K}\left(\mathfrak{a}_{\mathrm{q}}\right)$. We denote by ${ }^{10} \mathrm{C}$ the associated space define by (8.1), with ' $\mathcal{W}$ in place of $\mathcal{W}$. The associated Eisenstein and partial Eisenstein integrals are similarly indicated with a backprime.

Lemma 8.4 There exists a unique linear map $R:{ }^{\circ} \mathrm{C} \rightarrow{ }^{\circ} \mathrm{C}$ such that

$$
E^{\circ}(P: \nu: x)={ }^{\prime} E^{\circ}(P: \nu: x) \circ R,
$$

for all $x \in \mathrm{X}$ and generic $\nu \in \mathfrak{a}_{\mathrm{qC}}^{*}$. The map $R$ is an isometric isomorphism.

Proof: For every $w \in \mathcal{W}$, let ' $w$ denote the unique element of ' $\mathcal{W}$ that represents the same class in $W / W_{K \cap H}$. Then for every $w \in \mathcal{W}$ we may select an element $l_{w} \in K_{0}$ such that ' $w \in l_{w} w N_{K \cap H}\left(\mathfrak{a}_{q}\right)$. The right regular action $R_{l_{w}}: C^{\infty}(M) \rightarrow$ $C^{\infty}(M)$ induces a linear isomorphism $R_{w}$ from $C^{\infty}\left(M / M \cap w H w^{-1}: \tau_{0}\right)$ onto $C^{\infty}\left(M / M \cap{ }^{\prime} w H^{\prime} w^{-1}: \tau_{0}\right)$. Let $R:{ }^{\circ} \mathcal{C} \rightarrow{ }^{1} \mathcal{C}$ be the direct sum of the isomorphisms $R_{w}$, for $w \in \mathcal{W}$. Then obviously $R$ is an isometry. Let $\psi \in{ }^{\circ} \mathcal{C}$. Then 
by Proposition 8.1 the map $g: \nu \mapsto^{'} E^{\circ}(P: \nu: x) \circ R \psi$ belongs to $\mathcal{E}_{P}^{\text {hyp }}(\mathrm{X}: \tau)$. Moreover, it follows from the same proposition that

$$
q_{\nu-\rho_{P}}\left(P,{ }^{\prime} w \mid g_{\nu}, \cdot, m\right)=(R \psi){ }_{w}(m),
$$

for $\nu \in \operatorname{reg}_{a}(g), w \in \mathcal{W}$ and $m \in M$. Also, by the definition of $R$,

$$
(R \psi)_{{ } w}(m)=\psi_{w}\left(m l_{w}\right),
$$

for each $w \in \mathcal{W}$ and all $m \in M$.

On the other hand, still by Proposition 8.1 the map $f: \nu \mapsto E^{\circ}(P: \nu: x) \psi$ belongs to $\mathcal{E}_{P}^{\text {hyp }}(\mathrm{X}: \tau)$ as well, and for $\nu \in \operatorname{reg}_{a}(f), w \in \mathcal{W}$ and $m \in M$,

$$
q_{\nu-\rho_{P}}\left(P, w \mid f_{\nu}, \cdot, m\right)=\psi_{w}(m) .
$$

This equation remains valid if we replace $w$ in the expression on the left-hand side by any element $\bar{w}$ of $w N_{K \cap H}\left(\mathfrak{a}_{\mathrm{q}}\right)$, see [14, Lemma 3.7. Now ' $w=l_{w} \bar{w}$, for some $\bar{w} \in w N_{K \cap H}\left(\mathfrak{a}_{\mathrm{q}}\right)$; hence, applying [14, Lemma 3.6, with $v=\bar{w}$ and $u=l_{w}$, we obtain that

$$
q_{\nu-\rho_{P}}\left(P,{ }^{\prime} w \mid f_{\nu}, \cdot, m\right)=\psi_{w}\left(m l_{w}\right) .
$$

Comparing (8.5) with (8.3) and (8.4) and applying the uniqueness statement of Proposition 8.1 we infer that $f=g$. On the other hand, if $R:{ }^{\circ} \mathrm{C} \rightarrow{ }^{\circ} \mathrm{C}$ is a linear map such that (8.2) is valid, then $f=g$, hence (8.3) and (8.5) are equal. This implies (8.4) and shows that $R$ is uniquely determined by the requirement (8.2).

Lemma 8.5 Let $P \in \mathcal{P}_{\sigma}^{\min }$ and $s \in W$. Then, for all $x \in \mathrm{X}_{+}, y \in \mathrm{X}$ and generic $\nu \in \mathfrak{a}_{\mathrm{qC}}^{*}$, the element

$$
E_{+, s}(P: \nu: x) E^{*}(P: \nu: y) \in \operatorname{End}\left(V_{\tau}\right)
$$

does not depend on the choice of $\mathcal{W}$ made in the text preceding [8.1).

Proof: Let $R$ be the isometry of Lemma 8.4 From the fact that the partial Eisenstein integrals are uniquely determined by the properties mentioned in the text above Lemma 8.4 it follows that they satisfy the transformation property (8.2) with on both sides $E^{\circ}$ replaced by $E_{+, s}$, for $s \in W$. See also [14, Lemma 2.2 .

On the other hand, taking adjoints of the homomorphisms on both sides of (8.2), and substituting $-\bar{\nu}$ for $\nu$, we obtain that

$$
E^{*}(P: \nu: x)=R^{*} \circ^{\prime} E^{*}(P: \nu: x),
$$

for all $x \in \mathrm{X}$ and generic $\nu \in \mathfrak{a}_{\mathrm{qC}}^{*}$. From the unitarity of $R$ it now follows that the endomorphism (8.6) does not change if we replace $E_{+, s}$ and $E^{*}$ by ${ }^{\prime} E_{+, s}$ and ' $E^{*}$, respectively. 
In the following we consider Eisenstein integrals associated with the standard parabolic subgroup $P_{0} \in \mathcal{P}_{\sigma}^{\min }$, but suppress the symbol $P_{0}$ in the notation. Moreover, we agree to write $E_{+}$for $E_{+, 1}$. We recall from [12, p. 41, that the meromorphic functions $\nu \mapsto E^{\circ}(\nu)$ and $\nu \mapsto E_{+}(\nu)$ have singular sets that are locally finite unions of real $\Sigma$-hyperplanes in $\mathfrak{a}_{\mathrm{qC}}^{*}$. Let $\mathcal{H}$ be the collection of the singular hyperplanes for $E^{\circ}(\cdot)$ and $E_{+}(\cdot)$. Let $t \in \mathrm{WT}(\Sigma)$ be a $W$ invariant even residue weight, see the text following (2.1). Associated with the data $\Sigma^{+}, t$, we define, for each subset $F \subset \Delta$ and every element $\lambda \in{ }^{*} \mathfrak{a}_{F \mathrm{qC}}^{*}$, the residue operator $\operatorname{Res}_{\lambda+\mathfrak{a}_{F q}^{*}}^{t}$ as in $\S$ [ with respect to the normalization of the Lebesgue measures $d \lambda$ of $\mathfrak{a}_{\mathrm{q}}^{*}$ and $d \mu_{F}$ of $\mathfrak{a}_{F \mathrm{q}}^{*}$ given at the end of that section.

The data $\Sigma^{+}, F$ and $\mathcal{H}$ determine a finite subset $\Lambda(F)$ of $-\mathbb{R}_{+} F$, the negative of the closed cone spanned by $F$, see [12, Eqn. (5.1), where between the set brackets the requirement 'for some $t$ ' should be added. We now recall, from 12], Eqn. (5.7), the definition of the kernel $K_{F}^{t}(\nu: x: y) \in \operatorname{End}\left(V_{\tau}\right)$, for $(x, y) \in$ $\mathrm{X}_{+} \times \mathrm{X}$ and generic $\nu \in \mathfrak{a}_{F \mathrm{qc}}^{*}$, by

$$
K_{F}^{t}(\nu: x: y)=\sum_{\lambda \in \Lambda(F)} \operatorname{Res}_{\lambda+\mathfrak{a}_{F q}^{*}}^{t}\left(\sum_{s \in W^{F}} E_{+}(s \cdot: x) \circ E^{*}(s \cdot: y)\right)(\lambda+\nu) .
$$

From the definition it follows that $\nu \mapsto K_{F}^{t}(\nu: \cdot: \cdot)$ is a meromorphic function on $\mathfrak{a}_{F \mathrm{qC}}^{*}$ with values in the space $C^{\infty}\left(\mathrm{X}_{+} \times \mathrm{X}: \tau \otimes \tau^{*}\right)$ and with singularities along the hyperplanes of a real $\Sigma_{r}(F)$-configuration. Here $\tau \otimes \tau^{*}$ denotes the tensor product representation of $K \times K$ in $\operatorname{End}\left(V_{\tau}\right) \simeq V_{\tau} \otimes V_{\tau}^{*}$.

The residue operators in (8.7) depend on the choices of $d \lambda$ and $d \mu_{F}$, see the discussion in $\S$ 罒 therefore, so does the kernel $K_{F}^{t}$.

Lemma 8.6 Let the data $\left(G, H, K, \tau, \mathfrak{a}_{\mathrm{q}}, \Sigma^{+}\right)$be fixed as above. Let $F \subset \Delta$ and let $t \in \mathrm{WT}(\Sigma)$ be a $W$-invariant even residue weight. These data completely determine $K_{F}^{t} d \mu_{F} d y$, the product of the kernel $K_{F}^{t}$, defined by 8.7), with the product measure $d \mu_{F} d y$ on $i \mathfrak{a}_{F q}^{*} \times \mathrm{X}$.

In particular, $K_{F}^{t} d \mu_{F} d y$ is independent of the particular choice of $\mathcal{W}$, made in the text preceding 8.1), and of the choices of B and dy, made in Sections 2 and 囵.

Proof: Put

$$
k_{F}(\cdot)=\sum_{s \in W^{F}} E_{+}(s \cdot: x) \circ E^{*}(s \cdot: y) .
$$

It follows from Lemma 8.5 that $k_{F}$ depends only on the data mentioned, and not on $\mathcal{W}, B, d y$. Moreover, from (8.7) and (5.4), it follows that

$$
K_{F}^{t}(\nu: x: y) d \mu_{F} d y=\sum_{\lambda \in \Lambda(F)} \underline{\operatorname{Res}}_{\lambda+\mathfrak{a}_{F q}^{*}}^{t}\left(k_{F}(\cdot+\nu)\right)(d \lambda) d y .
$$

The occurring residue operators only depend on the data mentioned, and the product measure $d \lambda d y$ only depends on the choice of $\left(G, H, K, \mathfrak{a}_{\mathrm{q}}\right)$, by the discussion in $\S$. All assertions now follow. 
Remark 8.7 Since $d \mu_{F}$ and $d y$ do not depend on the choice of $\mathcal{W}$, it follows that the same holds for the kernel $K_{F}^{t}$. This fact has already silently been exploited in [12], text below Lemma 8.1, where the choice of $\mathcal{W}$ is adapted to the set $F$.

According to 12, Cor. 10.10, the kernel $K_{F}^{t}(\nu: x: y)$ extends smoothly to all of $\mathrm{X}$ in the variable $x$; more precisely, $K_{F}^{t}$ is a meromorphic $C^{\infty}\left(\mathrm{X} \times \mathrm{X}\right.$, $\left.\operatorname{End}\left(V_{\tau}\right)\right)$ valued function on $\mathfrak{a}_{F \mathrm{qc}}^{*}$ with singularities along real $\Sigma_{r}(F)$-hyperplanes.

From [12, Eqn. (5.5) and (5.8), we recall the definition of the linear operator $T_{F}^{t}$ from $C_{c}^{\infty}(\mathrm{X}: \tau)$ to $C^{\infty}\left(\mathrm{X}_{+}: \tau\right)$ by

$$
T_{F}^{t} f(x)=|W| t\left(\mathfrak{a}_{F \mathrm{q}}^{+}\right) \int_{\varepsilon_{F}+i \mathfrak{a}_{F \mathrm{q}}^{*}} \int_{X} K_{F}^{t}(\nu: x: y) f(y) d y d \mu_{F}(\nu),
$$

for $f \in C_{c}^{\infty}(\mathrm{X}: \tau)$ and $x \in \mathrm{X}_{+}$. Here $d \mu_{F}$ is the translate by $\varepsilon_{F}$ of the Lebesgue measure on $i \mathfrak{a}_{F q}^{*}$ normalized as in Section 5 Moreover, $\varepsilon_{F}$ is a point in the chamber $\mathfrak{a}_{F \mathfrak{q}}^{+}$, arbitrary but sufficiently close to the origin.

Remark 8.8 If $G$ has compact center modulo $H$, then $\mathfrak{a}_{\Delta q}=\{0\}$ and $t\left(\mathfrak{a}_{\Delta q}^{+}\right)=$ 1. Moreover, the above is to be understood so that integration relative to $d \mu_{\Delta}$ means evaluation in 0 ; also, $\varepsilon_{\Delta}=0$. In this case we agree to write $K_{\Delta}^{t}(x: y)=$ $K_{\Delta}^{t}(0: x: y)$, so that the formula for $T_{\Delta}^{t}$ becomes

$$
T_{\Delta}^{t} f(x)=|W| \int_{X} K_{\Delta}^{t}(x: y) f(y) d y
$$

for $f \in C_{c}^{\infty}(\mathrm{X}: \tau)$ and $x \in \mathrm{X}_{+}$.

In 12, Cor. 10.11, it is shown that in fact $T_{F}^{t}$ maps into $C^{\infty}(\mathrm{X}: \tau)$ and defines a continuous linear operator $C_{c}^{\infty}(\mathrm{X}: \tau) \rightarrow C^{\infty}(\mathrm{X}: \tau)$. Moreover, by 12, Thm. 1.2, it follows that

$$
I=\sum_{F \subset \Delta} T_{F}^{t} \quad \text { on } \quad C_{c}^{\infty}(\mathrm{X}: \tau)
$$

Lemma 8.9 Let $\left(G, H, K, \tau, \mathfrak{a}_{\mathrm{q}}, \Sigma^{+}\right)$and $(F, t)$ be data as in Lemma8.6. These data determine the operator $T_{F}^{t}$, defined by 8.8), completely.

Proof: This follows from Lemma 8.6

We finish this section with a discussion of how the kernels $K_{F}^{t}$ and the operators $T_{F}^{t}$ behave under isomorphisms of reductive symmetric spaces.

Let $\varphi: G \rightarrow$ ' $G$ be an isomorphism of reductive groups of Harish-Chandra's class, and put ' $H=\varphi(H),{ }^{\prime} K=\varphi(K),{ }^{\prime} \tau:=\tau \circ \varphi^{-1},{ }^{\prime} \mathfrak{a}_{\mathrm{q}}=\varphi\left(\mathfrak{a}_{\mathrm{q}}\right)$. Let ' $\Sigma$ be the root system of ' $\mathfrak{a}_{\mathrm{q}}$ in ' $\mathfrak{g}$ and let ' $W$ denote the associated Weyl group. The isomorphism $\varphi$ naturally induces the linear isomorphism $\mathfrak{a}_{\mathrm{qC}}^{*} \rightarrow{ }^{\prime} \mathfrak{a}_{\mathrm{qC}}^{*}$ given by

$$
\nu \mapsto{ }^{\prime} \nu:=\left.\nu \circ \varphi^{-1}\right|_{\mathfrak{a}_{\mathrm{q}}} .
$$


This isomorphism restricts to an isomorphism of root systems $\Sigma \rightarrow{ }^{\prime} \Sigma$. Let $\Sigma^{+}$ be a positive system for $\Sigma$ and ' $\Sigma^{+}$the corresponding positive system for ' $\Sigma$. Let $\Delta$ and ${ }^{\prime} \Delta$ be the collections of simple roots for $\Sigma^{+}$and ${ }^{\prime} \Sigma^{+}$, respectively. We denote by ' $F$ the image in ' $\Delta$ of a subset $F \subset \Delta$.

The map $\varphi$ also naturally induces a bijection from $\mathrm{WT}(\Sigma)$ onto $\mathrm{WT}\left({ }^{\prime} \Sigma\right)$, which we denote by $t \mapsto{ }^{\prime} t$. If $t \in \mathrm{WT}(\Sigma)$ is $W$-invariant and even, then ' $t \in$ $\mathrm{WT}\left({ }^{\prime} \Sigma\right)$ is ' $W$-invariant and even.

We put $\mathrm{X}=G / H$ and ' $\mathrm{X}={ }^{\prime} G /{ }^{\prime} H$. Then the map $\varphi$ factors to a diffeomorphism $\bar{\varphi}: \mathrm{X} \rightarrow{ }^{\prime} \mathrm{X}$. This diffeomorphism induces the isomorphism $\varphi_{*}: C^{\infty}(\mathrm{X}: \tau) \rightarrow$ $C^{\infty}\left({ }^{\prime} \mathrm{X}:{ }^{\prime} \tau\right)$, given by $f \mapsto f \circ \bar{\varphi}^{-1}$. It maps $C_{c}^{\infty}(\mathrm{X}: \tau)$ onto $C_{c}^{\infty}\left({ }^{\prime} \mathrm{X}:{ }^{\prime} \tau\right)$. We select invariant measures $d x$ and ' $d x$ on $\mathrm{X}$ and 'X, respectively. As in Section 5 . this choice determines Lebesgue measures $d \lambda$ and ' $d \lambda$ on $i \mathfrak{a}_{\mathrm{q}}^{*}$ and $i{ }^{\prime} \mathfrak{a}_{\mathrm{q}}^{*}$, respectively. As in Section 2 we fix bilinear forms $B$ and ' $B$ on $\mathfrak{g}$ and ' $\mathfrak{g}$, respectively. These choices determine densities $d \mu_{F}$ and $d \mu^{\prime} F$ on $i \mathfrak{a}_{F \mathrm{q}}^{*}$ and $i^{\prime} \mathfrak{a}^{*}{ }_{F \mathrm{q}}$, respectively.

Let $K_{\backslash}{ }_{F}^{t}$ be the analogue of the kernel $K_{F}^{t}$ for the data $\left({ }^{\prime} G,{ }^{\prime} H,{ }^{\prime} K,{ }^{\prime} \tau,{ }^{\prime} \mathfrak{a}_{\mathrm{q}},{ }^{\prime} \Sigma^{+}\right.$, $\left.{ }^{\prime} F,{ }^{\prime} t,{ }^{\prime} d x,{ }^{\prime} B\right)$ in place of $\left(G, H, K, \tau, \mathfrak{a}_{\mathrm{q}}, \Sigma^{+}, F, t, d x, B\right)$. Moreover, let $T_{F}^{\prime} t$ be the associated analogue of the operator $T_{F}^{t}$.

Lemma 8.10 Let notation be as above. Then

$$
K_{{ }^{\prime}}^{\prime}\left({ }^{\prime} \nu: \bar{\varphi}(x): \bar{\varphi}(y)\right) \varphi^{*}\left(d \mu_{F}\right) \bar{\varphi}^{*}\left({ }^{\prime} d y\right)=K_{F}^{t}(\nu: x: y) d \mu_{F} d y,
$$

for $x, y \in \mathrm{X}$ and generic $\nu \in \mathfrak{a}_{\mathrm{qC}}^{*}$. Moreover, the corresponding operators are related by

$$
T_{F}^{t} \circ \varphi_{*}=\varphi_{*} \circ T_{F}^{t} \quad \text { on } \quad C_{c}^{\infty}(\mathrm{X}: \tau) .
$$

Proof: In view of Lemma 8.6 it suffices to prove the identity 8.11) in case ' $\mathcal{W}$,' $d x$ and ' $B$ are compatible with $\mathcal{W}, d x$ and $B$, via $\varphi$. It then follows from the definition of the kernels that

$$
K_{{ }_{F}^{\prime}}^{\prime}\left({ }^{\prime} \nu: \bar{\varphi}(x): \bar{\varphi}(y)\right)=K_{F}^{t}(\nu: x: y),
$$

whence (8.11).

Equation (8.12) now follows by combining (8.11) with (8.8) and using the relations induced by $\varphi$ between the data associated with $G$ and ' $G$.

\section{The generalized Eisenstein integral}

In this section we shall use the vanishing theorem to give an alternative characterization of the generalized Eisenstein integral defined in 12, Def. 10.7. This characterization, which is in the spirit of Proposition 8.1 will be used throughout the paper.

For the moment we assume the $G$ has compact center modulo $H$. Then, with notation as in Remark 8.8 we define the space

$$
\mathcal{A}^{t}(\mathrm{X}: \tau):=\operatorname{span}\left\{K_{\Delta}^{t}(\cdot: y) v \mid y \in \mathrm{X}_{+}, v \in V_{\tau}\right\} .
$$


This space equals the space $\mathcal{C}_{\nu}$ of [12], Eqn. (10.2), with $F=\Delta$ and $\nu=0$. It is finite dimensional and consists of $\mathbb{D}(\mathrm{X})$-finite functions in $C^{\omega}(\mathrm{X}: \tau)$, see [12], Lemma 10.1. We adopt the new notation (9.1) in stead of $\mathcal{C}_{\nu}$ to avoid confusion with the space defined by 8.17).

Lemma 9.1 Let $G$ have compact center modulo $H$, and let $t \in \mathrm{WT}(\Sigma)$ be a $W$-invariant even residue weight. Then the space $\mathcal{A}^{t}(\mathrm{X}: \tau)$ equals the image $T_{\Delta}^{t}\left(C_{c}^{\infty}(\mathrm{X}: \tau)\right)$.

Proof: From [12], Lemma 10.2, it follows that $K_{\Delta}^{t}$ extends to a real analytic function $\mathrm{X} \times \mathrm{X} \rightarrow \operatorname{End}\left(V_{\tau}\right) \simeq V_{\tau} \otimes V_{\tau}^{*}$ which is $\tau \otimes \tau^{*}$-spherical.

By density of $\mathrm{X}_{+}$in $\mathrm{X}$, continuity of $K_{\Delta}^{t}$ and finite dimensionality of the space $\mathcal{A}^{t}(\mathrm{X}: \tau)$, it follows that the latter contains the function $K_{\Delta}^{t}(\cdot: y) v$ for every $y \in \mathrm{X}$ and $v \in V_{\tau}$. This implies that $T_{\Delta}^{t}$ maps $C_{c}^{\infty}(\mathrm{X}: \tau)$ into $\mathcal{A}^{t}(\mathrm{X}: \tau)$.

To see that the converse inclusion holds, suppose that $\xi$ is a linear functional of $\mathcal{A}^{t}(\mathrm{X}: \tau)$, vanishing on $\operatorname{im}\left(T_{\Delta}^{t}\right)$. Then it suffices to show that $\xi=0$.

For every $x \in \mathrm{X}$ and $\eta \in V_{\tau}^{*}$, let $\xi_{x, \eta}$ denote the linear functional $\varphi \mapsto$ $\eta(\varphi(x))$ on $\mathcal{A}^{t}(\mathrm{X}: \tau)$. The intersection of the kernels of these linear functionals, as $x \in \mathrm{X}$ and $\eta \in V_{\tau}^{*}$, is zero. Therefore, these linear functionals span the dual of $\mathcal{A}^{t}(\mathrm{X}: \tau)$, and we see that there exist $n \geq 1, x_{1}, \ldots, x_{n} \in \mathrm{X}$ and $\eta_{1}, \ldots, \eta_{n} \in V_{\tau}^{*}$, such that $\xi=\sum_{j=1}^{n} \xi_{x_{j}, \eta_{j}}$. In view of (8.9), the fact that $\xi$ vanishes on $\operatorname{im}\left(T_{\Delta}^{t}\right)$ implies that

$$
\sum_{j=1}^{n} \int_{\mathrm{X}} \eta_{j} K_{\Delta}^{t}\left(x_{j}: y\right) f(y) d y=0
$$

for all $f \in C_{c}^{\infty}(\mathrm{X}: \tau)$. By sphericality of $K_{\Delta}^{t}$ in the second variable, the above integral also vanishes for all functions $f \in C_{c}^{\infty}\left(\mathrm{X}, V_{\tau}\right)$. Hence, $\sum_{j} \eta_{j} K_{\Delta}^{t}\left(x_{j}: \cdot\right)=$ 0 as a function in $C^{\infty}\left(\mathrm{X}, V_{\tau}^{*}\right)$. It follows that $\xi\left(K_{\Delta}(\cdot: y) v\right)=0$, for all $y \in \mathrm{X}_{+}$ and $v \in V_{\tau}$. In view of (9.1), this implies that $\xi=0$.

We now assume that $G$ is arbitrary again. Let $F \subset \Delta$ and let $F \mathcal{W} \subset N_{K}\left(\mathfrak{a}_{\mathrm{q}}\right)$ be a choice of representatives for $W_{F} \backslash W / W_{K \cap H}$. If $t \in \mathrm{WT}(\Sigma)$ we denote by ${ }^{*} t$ the induced residue weight of $\Sigma_{F}$, see [11, Eqn. (3.16). Let $t$ be $W$-invariant and even; then ${ }^{*} t$ is $W_{F}$-invariant and even.

If $v \in{ }^{F} \mathcal{W}$, let $K_{F}^{*} t\left(\mathrm{X}_{F, v}: m: m^{\prime}\right)$, for $m, m^{\prime} \in \mathrm{X}_{F, v}$, denote the analogue of $K_{\Delta}^{t}$ for the symmetric space $\mathrm{X}_{F, v}$. Note that $M_{F}$ has a compact center, so that the discussion of the beginning of this section applies to $M_{F}$ in stead of $G$. In particular, the data $\left(M_{F}, H_{F}, K_{F}, \tau_{F},{ }^{*} \mathfrak{a}_{F \mathrm{q}}, \Sigma_{F}^{+},{ }^{*} t\right)$ determine the finite dimensional space

$$
\mathcal{A}^{*} t\left(\mathrm{X}_{F, v}: \tau_{F}\right)=\operatorname{span}\left\{K_{F}^{* t}\left(\mathrm{X}_{F, v}: \cdot: m^{\prime}\right) \mid m^{\prime} \in \mathrm{X}_{F, v,+}\right\} .
$$

Note that this space was denoted $\mathcal{C}_{F, v}$ in [12, Eqn. (10.7). We define

$$
\mathcal{A}_{F}^{* t}=\oplus_{v \in F} \mathcal{W} \quad \mathcal{A}^{*} t\left(\mathrm{X}_{F, v}: \tau_{F}\right)
$$

this formal direct sum was denoted $\mathcal{C}_{F}$ in [12, Def. 10.7. The natural projections and embeddings associated with the above direct sum are denoted by

$$
\operatorname{pr}_{F, v}: \mathcal{A}_{F}^{*} t \rightarrow \mathcal{A}^{*} t\left(\mathrm{X}_{F, v}: \tau_{F}\right) \quad \text { and } \quad \mathrm{i}_{F, v}: \mathcal{A}^{*} t\left(\mathrm{X}_{F, v}: \tau_{F}\right) \rightarrow \mathcal{A}_{F}^{* t},
$$


for $v \in{ }^{F} \mathcal{W}$. Given $\psi \in \mathcal{A}_{F}^{*} t$ we shall also write $\psi_{v}:=\operatorname{pr}_{F, v} \psi$.

The generalized Eisenstein integral $E_{F}^{\circ}(\psi: \nu)$, defined in [12], Def. 10.7, is a function in $C^{\infty}(\mathrm{X}: \tau)$ that depends linearly on $\psi \in \mathcal{A}_{F}^{*} t$ and meromorphically on $\nu \in \mathfrak{a}_{F \mathrm{qc}}^{*}$. We shall not repeat the definition here, but instead give a characterization based on the vanishing theorem, Theorem 6.11 The following result will allow us to show that $E_{F}^{\circ}(\psi: \cdot)$ belongs to the space of families $\mathcal{E}_{F}^{\text {hyp }}(\mathrm{X}: \tau)$ introduced in Definition 6.6 with $Q=P_{F}$. For its formulation, we recall some notation from [12], $\S 8$.

In the rest of this section we write $E^{\circ}(\lambda: x):=E^{\circ}\left(P_{0}: \lambda: x\right)$. Similarly, if $v \in{ }^{F} \mathcal{W}$, we write $E^{\circ}\left(\mathrm{X}_{1 F, v}: \nu: m\right)$ for the normalized Eisenstein integral of $\mathrm{X}_{1 F, v}$, associated with the minimal parabolic subgroup $M_{1 F} \cap P_{0}$. The analogue of the space ${ }^{\circ} \mathrm{C}$ for the latter Eisenstein integral is

$$
{ }^{\circ} \mathcal{C}_{F, v}:=\oplus_{w \in \mathcal{W}_{F, v}} \quad C^{\infty}\left(M / M \cap w v H v^{-1} w^{-1}: \tau_{0}\right) .
$$

Here $\mathcal{W}_{F, v} \subset N_{M_{F} \cap K}\left(\mathfrak{a}_{\mathrm{q}}\right)$ is a choice of representatives for $W_{F} / W_{K_{F} \cap v H v^{-1}}$; see [12, Eqn. (8.2). Adapting the set $\mathcal{W}$ if necessary, we may assume that $\mathcal{W}_{F, v} \subset \mathcal{W}$. Then $\mathcal{W}$ is the disjoint union of the sets $\mathcal{W}_{F, v}$, for $v \in{ }^{F} \mathcal{W}$, see [12], Lemma 8.1. Accordingly, $\mathrm{i}_{F, v}$ denotes the natural inclusion ${ }^{\circ} \mathcal{C}_{F, v} \rightarrow{ }^{\circ} \mathcal{C}$, defined as the identity on each component of (9.3). Moreover,

$$
{ }^{\circ} \mathcal{C}=\oplus_{v \in F} \mathcal{W} \quad \mathrm{i}_{F, v}\left({ }^{\circ} \mathcal{C}_{F, v}\right) .
$$

We denote the associated projection operator ${ }^{\circ} \mathcal{C} \rightarrow{ }^{\circ} \mathcal{C}_{F, v}$ by $\operatorname{pr}_{F, v}$, for $v \in{ }^{F} \mathcal{W}$.

Lemma 9.2 Let $\mathcal{L}$ be a Laurent functional in $\mathcal{M}\left({ }^{*} \mathfrak{a}_{F \mathrm{qC}}^{*}, \Sigma_{F}\right)_{\text {laur }}^{*} \otimes{ }^{\circ} \mathcal{C}$. Then the family $g: \mathfrak{a}_{F \mathbf{q C}}^{*} \rightarrow C^{\infty}(\mathrm{X}: \tau)$, defined by

$$
g(\nu, x):=\mathcal{L}\left[E^{\circ}(\nu+\cdot: x)\right]
$$

belongs to $\mathcal{E}_{F, Y}^{\mathrm{hyp}}(\mathrm{X}: \tau)$, with $Y=\operatorname{supp} \mathcal{L}$. Moreover, if $v \in{ }^{F} \mathcal{W}$, then for $\nu$ in a dense open subset of $\mathfrak{a}_{F \mathrm{qC}}^{*}$,

$$
q_{\nu-\rho_{F}}\left(P_{F}, v \mid g_{\nu}, X, m\right)=\mathcal{L}\left[E^{\circ}\left(\mathrm{X}_{1 F, v}: \cdot+\nu: m\right) \circ \operatorname{pr}_{F, v}\right]
$$

for all $X \in \mathfrak{a}_{F \mathrm{q}}$ and $m \in \mathrm{X}_{F, v,+}$. Here $\operatorname{pr}_{F, v}$ denotes the projection associated with 9.4).

Proof: It suffices to prove the result for a Laurent functional of the form $\mathcal{L}=\mathcal{L}^{\prime} \otimes \psi$, with $\mathcal{L}^{\prime} \in \mathcal{M}\left({ }^{*} \mathfrak{a}_{F \mathrm{qC}}^{*}, \Sigma_{F}\right)_{\text {laur }}^{*}$ and $\psi \in{ }^{\circ} \mathcal{C}$. Define the family $f$ by $f(\nu: x):=E^{\circ}(\nu: x) \psi$.

It follows from [9], p. 52, Lemma 14, that there exists a locally finite collection $\mathcal{H}$ of $\Sigma$-hyperplanes in $\mathfrak{a}_{\mathrm{qC}}^{*}$ and a map $d: \mathcal{H} \rightarrow \mathbb{N}$ such that $f$ belongs to $\mathcal{M}\left(\mathfrak{a}_{\mathrm{qc}}^{*}, \mathcal{H}, d, C^{\infty}(\mathrm{X}: \tau)\right)$. From [14], Lemma 13.1, applied with $Q=P_{F}$, it follows that $g$ is a meromorphic function on $\mathfrak{a}_{F \mathrm{qc}}^{*}$ with values in $C^{\infty}(\mathrm{X}: \tau)$.

It follows from [14, Lemma 14.5, that there exists a $\delta \in D_{P}$ such that $f$ belongs to $\mathcal{E}_{0}^{\text {hyp }}\left(\mathrm{X}_{+}: \tau: \delta\right)_{\text {hglob }}$, see [14, Def. 13.10, for the definition of the latter 
space. According to 14, Thm. 13.12, this implies that $g \in \mathcal{E}_{F, Y}^{\text {hyp }}\left(\mathrm{X}_{+}: \tau\right)_{\text {glob }}$. We conclude that $g \in \mathcal{E}_{F, Y}^{\text {hyp }}(\mathrm{X}: \tau)$.

The family $f$ equals the family $f_{W}$ defined in [14, Prop. 15.4. It follows from that proposition, applied with $Q=P_{F}$ and with $\mathcal{L}^{\prime}$ in place of $\mathcal{L}$, that (9.5) holds for each $v \in{ }^{F} \mathcal{W}$, generic $\nu \in \mathfrak{a}_{F \mathbf{q C}}^{*}$ and all $X \in \mathfrak{a}_{F \mathrm{q}}^{*}$ and $m \in \mathrm{X}_{F, v,+}$. Combining this with [14, Theorem 7.7, Eqn. (7.14), we see that (9.5) holds for all $\nu$ in a dense open subset of $\mathfrak{a}_{F \mathrm{qc}}^{*}$, every $v \in{ }^{F} \mathcal{W}, X \in \mathfrak{a}_{F \mathrm{q}}^{*}$ and all $m \in \mathrm{X}_{F, v,+}$.

Theorem 9.3 Let $\psi \in \mathcal{A}_{F}^{*}$. Then $g: \nu \mapsto E_{F}^{\circ}(\psi: \nu)$ is the unique family in $\mathcal{E}_{F}^{\text {hyp }}(\mathrm{X}: \tau)$ with the following property. For all $\nu$ in some non-empty open subset of $\mathfrak{a}_{F \mathbf{q C}}^{*}$ and each $u \in{ }^{F} \mathcal{W}$,

$$
q_{\nu-\rho_{F}}\left(P_{F}, u \mid g_{\nu}\right)(X, m)=\psi_{u}(m), \quad\left(X \in \mathfrak{a}_{F \mathbf{q}}, m \in \mathrm{X}_{F, v,+}\right) .
$$

Remark 9.4 If $F=\Delta$ and $G$ has compact center modulo $H$, then $\mathfrak{a}_{F \mathrm{q}}=\{0\}$ and $f \mapsto f_{0}$ defines a bijection from $\mathcal{E}_{F}^{\text {hyp }}(\mathrm{X}: \tau)$ onto $\mathcal{A}(\mathrm{X}: \tau)$, the space of $\mathbb{D}(\mathrm{X})$-finite functions in $C^{\infty}(\mathrm{X}: \tau)$, see Remark 6.8 Moreover, ${ }^{F} \mathcal{W}$ consists of one element which one may take to be $1, M_{F} / M_{F} \cap H \simeq \mathrm{X}$, and $\mathcal{A}_{F}^{*} \simeq \mathcal{A}^{t}(\mathrm{X}: \tau)$. Finally, with notation as in the above theorem, $g_{0}=\psi$, so that $\psi \mapsto E_{F}^{\circ}(\psi: 0)$ is the inclusion map $\mathcal{A}^{t}(\mathrm{X}: \tau) \rightarrow \mathcal{A}(\mathrm{X}: \tau)$.

Remark 9.5 In the proof of Theorem 9.3 we will encounter the set

$$
\Lambda\left(\mathrm{X}_{F, v}, F\right) \subset-\mathbb{R}_{+} F,
$$

which is defined to be the analogue of the set $\Lambda(\Delta)$ of (8.7), for the data $\left(\mathrm{X}_{F, v},{ }^{*} \mathfrak{a}_{F \mathfrak{q}}, \Sigma_{F}^{+}\right)$in place of $\left(\mathrm{X}, \mathfrak{a}_{\mathrm{q}}, \Sigma^{+}\right)$.

Proof: Uniqueness follows from the vanishing theorem, Theorem 6.11 hence, it suffices to prove existence.

In view of (9.2), we may assume that $\psi \in \mathcal{A}^{*} t\left(\mathrm{X}_{F, v}: \tau_{F}\right)$ for some $v \in{ }^{F} \mathcal{W}$. According to [12, Eqn. (10.9), we may then express $\psi$ as follows

$$
\psi(m)=\sum_{\lambda \in \Lambda\left(\mathrm{X}_{F, v}, F\right)} \operatorname{Res}_{\lambda}^{* t}\left[E^{\circ}\left(\mathrm{X}_{F, v}:-\cdot: m\right) \Phi(\cdot)\right], \quad\left(m \in \mathrm{X}_{F, v}\right),
$$

where

$$
\Phi(\lambda)=\sum_{j=1}^{k} E_{+}^{*}\left(\mathrm{X}_{F, v}:-\lambda: m_{j}\right) v_{j} \in{ }^{\circ} \mathcal{C}_{F, v}, \quad\left(\lambda \in{ }^{*} \mathfrak{a}_{F \mathbf{q C}}^{*}\right),
$$

with $\left\{m_{1}, \ldots, m_{k}\right\}$ a finite subset of $\mathrm{X}_{F, v,+}$, and $\left\{v_{1}, \ldots, v_{k}\right\}$ a finite subset of $V_{\tau}$. We now note that

$$
\mathcal{R}_{F}:=\sum_{\lambda \in \Lambda\left(\mathrm{X}_{F, v}, F\right)} \operatorname{Res}_{\lambda}^{*} t
$$


is a Laurent functional in $\mathcal{M}\left({ }^{*} \mathfrak{a}_{F \mathrm{qC}}^{*}, \Sigma_{F}\right)_{\text {laur }}^{*}$; moreover, according to [12, Def. 10.7, the generalized Eisenstein integral is given by

$$
g(\nu, x)=\mathcal{R}_{F}\left[E^{\circ}(\nu-\cdot: x) \circ \mathrm{i}_{F, v} \Phi(\cdot)\right] .
$$

Define the Laurent functional $\mathcal{L}_{0} \in \mathcal{M}\left({ }^{*} \mathfrak{a}_{F \mathrm{q}}^{*}, \Sigma_{F}\right)_{\text {laur }}^{*} \otimes{ }^{\circ} \mathcal{C}$ by

$$
\mathcal{L}_{0} \varphi:=\mathcal{R}_{F}\left[\varphi(-\cdot) \circ \mathrm{i}_{F, v} \Phi(\cdot)\right],
$$

for $\varphi \in \mathcal{M}\left({ }^{*} \mathfrak{a}_{F \mathfrak{q C}}^{*}, \Sigma_{F}\right) \otimes{ }^{\circ} \mathcal{C}^{*}$. Then the generalized Eisenstein integral is given by

$$
g(\nu, x)=\mathcal{L}_{0}\left[E^{\circ}(\nu+\cdot: x)\right]
$$

It now follows from Lemma 9.2 that $g \in \mathcal{E}_{F}^{\text {hyp }}(\mathrm{X}: \tau)$ and that, for $\nu$ in an open dense subset of $\mathfrak{a}_{F \mathbf{q C}}^{*}$ and all $X \in \mathfrak{a}_{F \mathbf{q}}$ and $m \in \mathrm{X}_{F, v,+}$,

$$
\begin{aligned}
q_{\nu-\rho_{F}}\left(P_{F}, u \mid g_{\nu}, X, m\right) & =\mathcal{L}_{0}\left[E^{\circ}\left(\mathrm{X}_{F, v}: \nu+\cdot: m\right) \circ \operatorname{pr}_{F, u}\right] \\
& =\mathcal{R}_{F}\left[E^{\circ}\left(\mathrm{X}_{F, v}: \nu-\cdot: m\right) \circ \operatorname{pr}_{F, u} \circ \mathrm{i}_{F, v} \Phi(\cdot \varnothing \oint . .11)\right.
\end{aligned}
$$

If $u \neq v$, then the latter expression equals 0 . Since also $\psi_{u}=0$, the identity (9.6) then follows. On the other hand, if $u=v$, then $\operatorname{pr}_{F, u} \circ \mathrm{i}_{F, v} \Phi(\cdot)=\Phi(\cdot)$; hence, (9.11) equals the expression on the right-hand side of (9.8), and since $\psi_{v}=\psi$, the identity (9.6) follows.

Corollary 9.6 There exists a locally finite collection $\mathcal{H}_{0}$ of hyperplanes in $\mathfrak{a}_{F \mathrm{qC}}^{*}$ such that the following holds. Let $\psi \in \mathcal{A}_{F}^{*}$ and let $g$ be defined as in Theorem 9.3. Then the meromorphic function $\nu \mapsto g_{\nu}$ is regular on the complement of $\cup \mathcal{H}_{0}$. Moreover, for every $u \in{ }^{F} \mathcal{W}, X \in \mathfrak{a}_{F \mathrm{q}}$ and $m \in \mathrm{X}_{F, v,+}$, formula (9.6) holds for all $\nu \in \mathfrak{a}_{F \mathrm{qC}}^{*} \backslash \cup \mathcal{H}_{0}$.

Proof: Let $\overline{1}$ denote the image of 1 in $W / \sim_{P_{F} \mid P_{F}}$, see (7.1). Let

$$
\nu \mapsto q_{\overline{1}, 0}\left(P_{F}, u \mid g\right)(\nu, X, m)
$$

be the function in $\mathcal{M}\left(\mathfrak{a}_{F \mathrm{qC}}^{*}, \Sigma_{r}(F), V_{\tau}\right)$, defined as in Proposition [7.1] By [14, Thm. 7.7, there exists a locally finite collection $\mathcal{H}_{0}$ of hyperplanes in $\mathfrak{a}_{F \mathrm{qc}}^{*}$ such that $\nu \mapsto g_{\nu}$ is regular on $\mathfrak{a}_{F \mathrm{qc}}^{*} \backslash \cup \mathcal{H}_{0}$ and for every $u \in{ }^{F} \mathcal{W}$, all $X \in \mathfrak{a}_{F \mathrm{q}}$ and $m \in \mathrm{X}_{F, u,+}$,

$$
q_{\overline{1}, 0}\left(P_{F}, u \mid g\right)(\nu, X, m)=q_{\nu-\rho_{F}}\left(P_{F}, u \mid g_{\nu}, X, m\right),
$$

for all $\nu \in \mathfrak{a}_{F \mathrm{qC}}^{*} \backslash \cup \mathcal{H}_{0}$. By linearity in $\psi$ and finite dimensionality of the space $\mathcal{A}_{F}^{*}$, the collection $\mathcal{H}_{0}$ can be chosen independent of $\psi$. Combination of (9.13) and (9.6) gives that the meromorphic function (9.12) is constant and equal to $\psi_{u}(m)$. In view of (9.13) it now follows that (9.6) holds for all $X \in \mathfrak{a}_{F \mathrm{q}}$, $m \in \mathrm{X}_{F, u,+}$ and $\nu \in \mathfrak{a}_{F \mathrm{qC}}^{*} \backslash \cup \mathcal{H}_{0}$. 
From the uniqueness statement in Theorem 9.3 it follows that the generalized Eisenstein integral $E_{F}^{\circ}(\psi) \in \mathcal{E}_{F}^{\text {hyp }}(\mathrm{X}: \tau)$ depends linearly on $\psi \in \mathcal{A}_{F}^{* t}$. We agree to write $E_{F}^{\circ}(\nu: x) \psi:=E_{F}^{\circ}(\psi: \nu: x)$, for $x \in \mathrm{X}$ and generic $\nu \in \mathfrak{a}_{F \mathrm{qc}}^{*}$. Accordingly, we view the generalized Eisenstein integral as a meromorphic function on $\mathfrak{a}_{F \mathrm{qC}}^{*}$ with values in $C^{\infty}(\mathrm{X}: \tau \otimes 1)$; here $\tau \otimes 1$ denotes the tensor product representation in $\operatorname{Hom}\left(\mathcal{A}_{F}^{*} t, V_{\tau}\right) \simeq V_{\tau} \otimes\left(\mathcal{A}_{F}^{*} t\right)^{*}$. In accordance with [14, Def. 10.7, we put

$$
E_{F, v}^{\circ}(\nu: x):=E_{F}^{\circ}(\nu: x) \circ \mathrm{i}_{F, v} \in \operatorname{Hom}\left(\mathcal{A}^{*} t\left(\mathrm{X}_{F, v}: \tau_{F}\right), V_{\tau}\right),
$$

for $v \in{ }^{F} \mathcal{W}, x \in \mathrm{X}$ and generic $\nu \in \mathfrak{a}_{F \mathrm{qC}}^{*}$.

Lemma 9.7 Let $v \in{ }^{F} \mathcal{W}$ and let $\psi \in \mathcal{A}^{*} t\left(\mathrm{X}_{F, v}: \tau_{F}\right)$.

(a) There exists a Laurent functional $\mathcal{L} \in \mathcal{M}\left({ }^{*} \mathfrak{a}_{F \mathrm{qC}}^{*}, \Sigma_{F}\right)_{\text {laur }}^{*} \otimes{ }^{\circ} \mathcal{C}_{F, v}$ such that

$$
\psi(m)=\mathcal{L}\left[E^{\circ}\left(\mathrm{X}_{F, v}: \cdot: m\right)\right], \quad\left(m \in \mathrm{X}_{F, v}\right) .
$$

(b) There exists a functional as in (a) with support contained in $\Lambda\left(\mathrm{X}_{F, v}, F\right)$, the set introduced in Remark 9.5. In particular, the support of this functional is real.

(c) If $\mathcal{L}$ is any Laurent functional as in (a), then, for all $x \in \mathrm{X}$,

$$
E_{F, v}^{\circ}(\nu: x) \psi=\mathcal{L}\left[E^{\circ}(\nu+\cdot: x) \circ \mathrm{i}_{F, v}\right]
$$

as an identity of meromorphic functions in $\nu \in \mathfrak{a}_{F \mathrm{qC}}^{*}$.

Proof: As in the proof of Theorem 9.3 we may express $\psi$ by $\left(9.8\right.$. Let $\mathcal{L}_{0}$ be defined as in (9.10) and let the Laurent functional $\mathcal{L} \in \mathcal{M}\left({ }^{*} \mathfrak{a}_{F \mathrm{qC}}^{*}, \Sigma_{F}\right)_{\text {laur }}^{*} \otimes{ }^{\circ} \mathcal{C}_{F, v}$ be defined by $\mathcal{L} \varphi=\mathcal{L}_{0}\left(\varphi(\cdot) \circ \operatorname{pr}_{F, v}\right)$, for $\varphi \in \mathcal{M}\left({ }^{*} \mathfrak{a}_{F \mathrm{qC}}^{*}, \Sigma_{F}\right) \otimes{ }^{\circ} \mathcal{C}_{F, v}^{*}$. Since $\operatorname{pr}_{F, v} \circ \mathrm{i}_{F, v}=I$ on ${ }^{\circ} \mathcal{C}_{F, v}$, it follows from (9.10), (9.9) and (9.8) that $\mathcal{L}$ satisfies (9.15). We observe that $\operatorname{supp} \mathcal{L} \subset \operatorname{supp} \mathcal{R}_{F} \subset \Lambda\left(X_{F, v}, F\right)$; in particular, $\mathcal{L}$ has support contained in ${ }^{*} \mathfrak{a}_{F \mathrm{q}}^{*}$. This establishes (a) and (b).

Now assume that $\mathcal{L}$ is a Laurent functional as in (a). Let $\mathcal{L}^{\prime}$ be the Laurent functional in $\mathcal{M}\left({ }^{*} \mathfrak{a}_{F \mathrm{gc}}^{*}, \Sigma_{F}\right)_{\text {laur }}^{*} \otimes{ }^{\circ} \mathcal{C}$ defined by $\mathcal{L}^{\prime} \varphi=\mathcal{L}\left(\varphi(\cdot) \circ \mathrm{i}_{F, v}\right)$. Then it follows from Lemma 9.2 that the family $g: \mathfrak{a}_{F \mathrm{qc}}^{*} \times \mathrm{X} \rightarrow V_{\tau}$ defined by

$$
g(\nu, x)=\mathcal{L}^{\prime}\left[E^{\circ}(\nu+\cdot: x)\right]
$$

belongs to $\mathcal{E}_{F}^{\text {hyp }}(\mathrm{X}: \tau)$ and satisfies, for $u \in{ }^{F} \mathcal{W}, \nu$ in an open dense subset of $\mathfrak{a}_{F \mathrm{qC}}^{*}$ and all $X \in \mathfrak{a}_{F \mathrm{q}}$ and $m \in \mathrm{X}_{F, u}$,

$$
\begin{aligned}
q_{\nu-\rho_{F}}\left(P_{F}, u \mid g_{\nu}\right) & =\mathcal{L}^{\prime}\left[E^{\circ}\left(\mathrm{X}_{F, v}: \cdot\right) \circ \operatorname{pr}_{F, u}\right] \\
& =\mathcal{L}\left[E^{\circ}\left(\mathrm{X}_{F, v}: \cdot\right) \circ \operatorname{pr}_{F, u} \circ \mathrm{i}_{F, v}\right] \\
& =\operatorname{pr}_{F, u} \circ \mathrm{i}_{F, v} \psi .
\end{aligned}
$$

Here we note that the last equality is obvious for $u \neq v$, since then $\operatorname{pr}_{F, u} \circ \mathrm{i}_{F, v}=$ 0 . On the other hand, if $u=v$, then $\operatorname{pr}_{F, u} \circ \mathrm{i}_{F, v}=I$ on $\mathcal{A}^{*} t\left(\mathrm{X}_{F, v}: \tau_{F}\right)$ and the equality follows from the assumption on $\psi$. It now follows from Theorem 9.3 that $g(\nu, x)=E_{F}^{\circ}(\nu: x) \circ \mathrm{i}_{F, v} \psi$. 
Combining the above result with Lemma 8.3 we obtain the following information on the asymptotic coefficients of the generalized Eisenstein integral. We put

$$
\mathrm{Y}(F):=\cup_{v \in F \mathcal{W}} \Lambda\left(\mathrm{X}_{F, v}, F\right) .
$$

This is a finite subset of $-\mathbb{R}_{+} F$, which in turn is contained in ${ }^{*} \mathfrak{a}_{F \mathrm{q}}^{*}$.

Lemma 9.8 Let $F \subset \Delta$ and $\psi \in \mathcal{A}_{F}^{*}$. The family $f:(\nu, x) \mapsto E_{F}^{\circ}(\nu: x) \psi$ belongs to $\mathcal{E}_{F, \mathrm{Y}(F)}^{\text {hyp }}(\mathrm{X}: \tau)$. Moreover, the $\Sigma_{r}(F)$-configuration $\mathcal{H}_{f}$, defined in the text preceding Definition 6.3, is real.

Put $k=\operatorname{deg}_{a} f$ and let $Q \in \mathcal{P}_{\sigma}, u \in N_{K}\left(\mathfrak{a}_{\mathrm{q}}\right)$. Then, for every $\sigma \in W / \sim_{Q \mid P_{F}}$ and all $\xi \in-\sigma \cdot \mathrm{Y}(F)+\Delta_{r}(Q)$,

$$
q_{\sigma, \xi}(Q, u \mid f) \in P_{k}\left(\mathfrak{a}_{Q \mathrm{q}}\right) \otimes \mathcal{M}\left(\mathfrak{a}_{F \mathrm{qc}}^{*}, \mathcal{H}_{f}, d_{f}, C^{\infty}\left(\mathrm{X}_{Q, u}: \tau_{Q}\right)\right) .
$$

Proof: From Theorem 9.3 it follows that $f \in \mathcal{E}_{F, Y}^{\text {hyp }}(\mathrm{X}: \tau)$, with $Y$ a finite subset of ${ }^{*} \mathfrak{a}_{F \mathrm{qc}}^{*}$.

For the first two assertions we may assume that $f_{\nu}=E_{F, v}^{\circ}(\nu) \psi$, with $\psi \in$ $\mathcal{A}^{*} t\left(\mathrm{X}_{F, v}: \tau_{F}\right)$. Select $\mathcal{L} \in \mathcal{M}\left({ }^{*} \mathfrak{a}_{F \mathrm{qC}}^{*}, \Sigma_{F}\right)_{\text {laur }}^{*} \otimes{ }^{\circ} \mathcal{C}_{F, v}$ as in Lemma 9.7 (b). According to Lemma 8.3 there exists a real $\Sigma$-configuration $\mathcal{H}$ in $\mathfrak{a}_{\mathrm{qC}}^{*}$ such that for every $\psi^{\prime} \in{ }^{\circ} \mathcal{C}_{F, v}$, the family $g: \lambda \mapsto E^{\circ}(\lambda) \mathrm{i}_{F, v} \psi^{\prime}$, which belongs to $\mathcal{E}_{0}^{\text {hyp }}(\mathrm{X}: \tau)$, satisfies $\mathcal{H}_{g} \subset \mathcal{H}$. It now follows from Lemma 9.7 (c), combined with 14, Prop. 13.2, that $Y \subset Y(F)$ and that $\mathcal{H}_{f} \subset \mathcal{H}_{F}(Y(F))$, with the latter set defined as in 14], Eqn. (11.6). It follows from the mentioned definition and the fact that $\mathcal{H}$ and $Y(F)$ are real, that $\mathcal{H}_{F}(Y(F))$ and hence $\mathcal{H}_{f}$ are real. It remains to establish (9.17).

Let $Q, u, \sigma$ be as asserted. With a reasoning as above, it follows from Lemma 9.7 combined with 14, Lemma 14.5 and Proposition 13.9, that $f$ is holomorphically $\sigma$-global along $(Q, u)$ (see 14, Definition 13.6). Let $\xi \in-\sigma \cdot Y+\mathbb{N} \Delta_{r}(Q)$. Then (9.17) follows by application of 14, Proposition 13.8 .

\section{Temperedness of the Eisenstein integral}

In this section we show that the generalized Eisenstein integral $E_{F}^{\circ}(\nu) \psi$, defined in the previous section, is tempered for regular values of $\nu$ in $i \mathfrak{a}_{F \mathrm{q}}^{*}$.

Let us first recall the notion of temperedness. Following [5], p. 415, we define the function $\Theta: \mathrm{X} \rightarrow \mathbb{R}$ by

$$
\Theta(x)=\sqrt{\Xi\left(x \sigma(x)^{-1}\right)},
$$

where $\Xi$ is the elementary spherical function $\varphi_{0}$ associated with the Riemannian symmetric space $G / K$.

Moreover, we define the function $l_{\mathrm{X}}: \mathrm{X} \rightarrow[0, \infty[$, denoted $\tau$ in [5], by

$$
l_{\mathrm{X}}(k a h)=|\log a|, \quad\left(k \in K, a \in A_{\mathrm{q}}, h \in H\right) .
$$


Definition 10.1 $\mathrm{A} \mathbb{D}(\mathrm{X})$-finite function $f$ in $C^{\infty}(\mathrm{X}: \tau)$ is said to be tempered if there exists a $d \in \mathbb{N}$ such that

$$
\sup _{\mathrm{X}}\left(1+l_{\mathrm{X}}\right)^{-d} \Theta^{-1}\|f\|<\infty .
$$

The space of these functions is denoted by $\mathcal{A}_{\text {temp }}(\mathrm{X}: \tau)$.

The following lemma gives a criterion for temperedness in terms of exponents. We assume that $\mathrm{P}^{\min } \subset \mathcal{P}_{\sigma}^{\min }$ is a choice of representatives for $\mathcal{P}_{\sigma}^{\min } / W_{K \cap H}$ and that $\mathcal{W} \subset N_{K}\left(\mathfrak{a}_{\mathrm{q}}\right)$ is a choice of representatives for $W / W_{K \cap H}$. We also assume that $P_{1}$ is a fixed element of $\mathcal{P}_{\sigma}^{\min }$.

Lemma 10.2 Let $f \in C^{\infty}(\mathrm{X}: \tau)$ be a $\mathbb{D}(\mathrm{X})$-finite function. Then the following conditions are equivalent.

(a) $f \in \mathcal{A}_{\text {temp }}(\mathrm{X}: \tau)$.

(b) For each $P \in \mathrm{P}^{\mathrm{min}}$ and every $\xi \in \operatorname{Exp}(P, e \mid f)$ the estimate $\operatorname{Re} \xi+\rho_{P} \leq 0$ holds on $\mathfrak{a}_{\mathrm{q}}^{+}(P)$.

(c) For each $v \in \mathcal{W}$ and every $\xi \in \operatorname{Exp}\left(P_{1}, v \mid f\right)$ the estimate $\operatorname{Re} \xi+\rho_{P_{1}} \leq 0$ holds on $\mathfrak{a}_{\mathrm{q}}^{+}\left(P_{1}\right)$.

Proof: By sphericality and the decomposition $G=\mathrm{cl} \cup_{P \in \mathrm{P} \min } K A_{\mathrm{q}}^{+}(P) H$, see [3], Cor. 1.4 and top of p. 232, the estimate (10.2) is equivalent to the requirement that, for each $P \in \mathrm{P}^{\text {min }}$,

$$
\sup _{a \in A_{q}^{+}(P)}(1+|\log a|)^{-d} \Theta(a)^{-1}\|f(a)\|<\infty .
$$

By [5], Prop. 17.2, there exist constants $C>0$ and $N \in \mathbb{N}$ such that, for each $P \in \mathrm{P}^{\mathrm{min}}$,

$$
a^{-\rho_{P}} \leq \Theta(a) \leq C(1+|\log a|)^{N} a^{-\rho_{P}} \quad\left(a \in A_{\mathbf{q}}^{+}(P)\right) .
$$

Therefore, (10.2) is equivalent to the existence of a constant $d^{\prime} \in \mathbb{N}$ such that, for each $P \in \mathrm{P}^{\mathrm{min}}$,

$$
\sup _{a \in A_{\mathrm{q}}^{+}(P)}(1+|\log a|)^{-d^{\prime}} a^{\rho_{P}}\|f(a)\|<\infty .
$$

According to 3], Thm. 6.1, this condition is in turn equivalent to (b). This establishes the equivalence of (a) and (b), for any choice of $\mathrm{P}^{\mathrm{min}}$. The equivalence of (b) and (c) follows from the observation that $\left\{v^{-1} P_{1} v \mid v \in \mathcal{W}\right\}$ is a choice of representatives for $\mathcal{P}_{\sigma}^{\min } / W_{K \cap H}$ combined with the fact that $\operatorname{Exp}\left(v^{-1} P_{1} v, e \mid\right.$ $f)=v^{-1} \operatorname{Exp}\left(P_{1}, v \mid f\right)$, for $v \in \mathcal{W}$, by [14, Lemma 3.6. 
If $G$ has compact center modulo $H$ and $t \in \mathrm{WT}(\Sigma)$ is a $W$-invariant even residue weight, then according to 12, Lemma 10.3, there exists, for every choice of Hilbert structure on the space $\mathcal{A}^{t}(\mathrm{X}: \tau)$, a unique endomorphism $\alpha=\alpha^{t}$ of this space, such that

$$
K_{\Delta}^{t}(x: y)=\mathbf{e}(x) \circ \alpha \circ \mathbf{e}(y)^{*},
$$

for $x, y \in \mathrm{X}_{+}$. Here the map $\mathbf{e}(x): \mathcal{A}^{t}(\mathrm{X}: \tau) \rightarrow V_{\tau}$ is defined by $\varphi \mapsto \varphi(x)$. The corresponding function $\mathbf{e}$, with values in $\operatorname{Hom}\left(\mathcal{A}^{t}(\mathrm{X}: \tau), V_{\tau}\right) \simeq V_{\tau} \otimes \mathcal{A}^{t}(\mathrm{X}: \tau)^{*}$, is a $\tau \otimes 1$-spherical real analytic function on $\mathrm{X}$. We recall from [12], Lemma 10.3, that $\alpha$ is self-adjoint and bijective.

In the following we assume that $t \in \mathrm{WT}(\Sigma)$ is a $W$-invariant even residue weight and that $F \subset \Delta$. We equip each finite dimensional space $\mathcal{A}^{*} t\left(\mathrm{X}_{F, v}: \tau_{F}\right)$, for $v \in{ }^{F} \mathcal{W}$, with a positive definite inner product. Moreover, we equip the direct sum space $\mathcal{A}_{F}^{* t}$, defined by (9.2), with the direct sum inner product, denoted $\langle\cdot \mid \cdot\rangle$. Here and in the following, we use a bar in the notation of an inner product to indicate its sesquilinearity. Moreover, all such inner products will be antilinear in the second variable.

Let $\alpha_{F, v}^{*}=\alpha_{F, v}$ be the analogue of the endomorphism $\alpha$ for $\left(\mathrm{X}_{F, v}, \tau_{F}\right)$, and let $\alpha_{F}^{*} t=\alpha_{F} \in \operatorname{End}\left(\mathcal{A}^{*} t\right)$ be the direct sum of the $\alpha_{F, v}$, for $v \in{ }^{F} \mathcal{W}$. Then $\alpha_{F}$ is self-adjoint and bijective. Moreover, according to [12], Prop. 10.9, see also Lemma 10.2, we have, for $x, y \in \mathrm{X}$,

$$
K_{F}^{t}(\nu: x: y)=E_{F}^{\circ}(\nu: x) \circ \alpha_{F} \circ E_{F}^{*}(\nu: y)
$$

as an identity of $\operatorname{End}\left(V_{\tau}\right)$-meromorphic functions in the variable $\nu \in \mathfrak{a}_{F \mathrm{qc}}^{*}$. Here $E_{F}^{*}$ denotes the dual generalized Eisenstein integral, defined by

$$
E_{F}^{*}(\nu: y):=E_{F}^{\circ}(-\bar{\nu}: y)^{*} \in \operatorname{Hom}\left(V_{\tau}, \mathcal{A}_{F}^{* t}\right),
$$

for $y \in \mathrm{X}$ and generic $\nu \in \mathfrak{a}_{F \mathrm{qC}}^{*}$.

Lemma 10.3 There exists a locally finite collection $\mathcal{H}_{1}$ of affine hyperplanes in $\mathfrak{a}_{F \mathrm{qc}}^{*}$, such that $\nu \mapsto E_{F}^{*}(\nu)$ is regular on $\mathfrak{a}_{F \mathrm{qC}}^{*} \backslash \cup \mathcal{H}_{1}$, and such that the following holds. For every $\nu \in \mathfrak{a}_{F \mathrm{qc}}^{*} \backslash \cup \mathcal{H}_{1}$,

$$
\operatorname{span}\left\{E_{F}^{*}(\nu: y) v \mid y \in \mathrm{X}_{+}, v \in V_{\tau}\right\}=\mathcal{A}_{F}^{*} .
$$

Proof: Let $\mathcal{H}_{0}$ be the collection of hyperplanes of Corollary [9.6 and let $\mathcal{H}_{1}$ be the image of $\mathcal{H}_{0}$ under the map $\nu \mapsto-\bar{\nu}$. In view of 10.5) the function $\nu \mapsto E_{F}^{*}(\nu)$ is regular on the complement of $\cup \mathcal{H}_{1}$.

Let $\nu \in \mathfrak{a}_{F \mathrm{qC}}^{*} \backslash \cup \mathcal{H}_{1}$ and let $\psi \in \mathcal{A}_{F}^{*} t$. Assume that

$$
\left\langle\psi \mid E_{F}^{*}(\nu: y) v\right\rangle=0 \quad \text { for all } \quad y \in \mathrm{X}_{+}, v \in V_{\tau} .
$$

Using (10.5) we see that $E_{F}^{\circ}(-\bar{\nu}) \psi=0$. It now follows from Corollary 9.6 that $\psi_{u}=0$ for each $u \in{ }^{F} \mathcal{W}$. Hence, $\psi=0$ and (10.6) follows. 
In the following we write $\rho=\rho_{P_{0}}$, where $P_{0}$ denotes the standard parabolic subgroup in $\mathcal{P}_{\sigma}^{\min }$.

Lemma 10.4 Let $v \in V_{\tau}$ and $y \in \mathrm{X}_{+}$. Then the family $f:(\nu, x) \mapsto K_{F}^{t}(\nu: x: y) v$ belongs to $\mathcal{E}_{F}^{\text {hyp }}(\mathrm{X}: \tau)$. Moreover, for every $\nu \in \operatorname{reg} f$ and each $u \in \mathcal{W}$,

$$
\operatorname{Exp}\left(P_{0}, u \mid f_{\nu}\right) \subset W^{F}(\nu+\Lambda(F))-\rho-\mathbb{N} \Delta,
$$

where $\Lambda(F)$ denotes the finite subset of $-\mathbb{R}_{+} F$ introduced in (8.7).

Proof: The first assertion follows from (10.4) and Theorem 9.3

According to 12, Prop. 3.1, the function $\lambda \mapsto E^{*}\left(P_{0}: \lambda: y\right) v$ belongs to the space $\mathcal{M}\left(\mathfrak{a}_{\mathrm{qc}}^{*}, \Sigma\right) \otimes^{\circ} \mathcal{C}(\tau)$. Combining this with [14, Lemma 14.3, we deduce that the family $h: \mathfrak{a}_{\mathrm{qC}}^{*} \times \mathrm{X}_{+} \rightarrow V_{\tau}$, defined by

$$
h(\lambda, x)=\sum_{s \in W^{F}} E_{+, s}\left(P_{0}: \lambda: x\right) E^{*}\left(P_{0}: \lambda: y\right) v
$$

belongs to $\mathcal{E}_{0}^{\text {hyp }}\left(\mathrm{X}_{+}: \tau\right)$, hence to $C_{0}^{\text {ep,hyp }}\left(\mathrm{X}_{+}: \tau\right)$. Using [14, Eqn. (14.13), we see that if $s \in W, \mu \in \mathbb{N} \Delta$ and $u \in N_{K}\left(\mathfrak{a}_{\mathrm{q}}\right)$, then

$$
q_{s, \mu}\left(P_{0}, u \mid h\right) \neq 0 \Rightarrow s \in W^{F} .
$$

In the notation of $\S$ 國 define the Laurent functional $\mathcal{L} \in \mathcal{M}\left({ }^{*} \mathfrak{a}_{F \mathrm{qc}}^{*}, \Sigma_{F}\right)_{\text {laur }}^{*}$ by

$$
\mathcal{L}=\sum_{\lambda \in \Lambda(F)} \operatorname{Res}_{\lambda+\mathfrak{a}_{F q}^{*}}^{t}
$$

then $\operatorname{supp} \mathcal{L} \subset \Lambda(F)$. It follows from (8.7) that $f=\mathcal{L}_{*} h$. From [14, Prop. 13.2 (b), it now follows that there exists an open dense subset $\Omega \subset \mathfrak{a}_{F \mathrm{qC}}^{*}$ such that, for $\nu \in \Omega$,

$\operatorname{Exp}\left(P_{0}, u \mid f_{\nu}\right) \subset\left\{s(\nu+\lambda)-\rho-\mu \mid s \in W, \lambda \in \Lambda(F), \mu \in \mathbb{N} \Delta, q_{s, \mu}\left(P_{0}, v \mid h\right) \neq 0\right\}$.

In view of (10.8) this implies that the inclusion (10.7) holds for $\nu \in \Omega$. From $f \in \mathcal{E}_{F}^{\text {hyp }}(\mathrm{X}: \tau)$ it follows in particular that there exists a finite subset $Y \subset{ }^{*} \mathfrak{a}_{F \mathrm{qC}}^{*}$ such that $f \in C_{F, Y}^{\text {ep,hyp }}\left(\mathrm{X}_{+}: \tau\right)$. The canonical map $W \rightarrow W / W_{F}$ restricts to a bijection $s \mapsto \bar{s}$ from $W^{F}$ onto $W / W_{F}$. For $s \in W^{F}$ we put $E_{\bar{s}}=-s \Lambda(F)+\mathbb{N} \Delta$. We now apply Lemma 7.2 with $Q=P_{F}, P=P_{0}$, so that $W / \sim_{P \mid Q} \simeq W / W_{F}$, and with $E_{\sigma}$ as just defined, for $\sigma \in W / W_{F}$. Then it follows that the inclusion (10.7) holds for $\nu \in \operatorname{reg} f$.

Theorem 10.5 Let $\psi \in \mathcal{A}_{F}^{* t}$ and $p \in \Pi_{\Sigma_{r}(F)}\left(\mathfrak{a}_{F \mathrm{q}}^{*}\right)$. Then $g:(\nu, x) \mapsto p(\nu) E_{F}^{\circ}(\nu: x) \psi$ defines a family in $\mathcal{E}_{F}^{\text {hyp }}(\mathrm{X}: \tau)$. Moreover, for each $v \in \mathcal{W}$ and every $\nu \in \operatorname{reg} g$,

$$
\operatorname{Exp}\left(P_{0}, v \mid g_{\nu}\right) \subset W^{F}(\nu+\Lambda(F))-\rho-\mathbb{N} \Delta,
$$

where $\Lambda(F)$ denotes the finite subset of $-\mathbb{R}_{+} F$ introduced above (8.7). 
Proof: The first assertion follows from Theorem 9.3 By Lemma 10.3 there exists a $\nu_{0} \in \mathfrak{a}_{F \mathrm{qC}}^{*}$ and elements $y_{j} \in \mathrm{X}_{+}$and $v_{j} \in V_{\tau}$, for $1 \leq j \leq r$, such that $\alpha_{F} \circ E_{F}^{*}\left(\nu_{0}: y_{j}\right) v_{j}, 1 \leq j \leq r$ is a basis for $\mathcal{A}_{F}^{* t}$. Define meromorphic $\mathcal{A}_{F}^{*} t$ valued functions on $\mathfrak{a}_{F \mathbf{q C}}^{*}$ by $\psi_{j}:=\alpha_{F} \circ E_{F}^{*}\left(\cdot: y_{j}\right) v_{j}$, for $1 \leq j \leq r$. By standard arguments involving analyticity and linear algebra it follows that $\left(\psi_{j}(\nu) \mid 1 \leq\right.$ $j \leq r)$ is a basis for $\mathcal{A}_{F}^{* t}$, for $\nu$ in an open dense subset of $\mathfrak{a}_{F \mathrm{qC}}^{*}$. Moreover, $\psi \in \mathcal{A}_{F}^{* t}$ may be expressed as a linear combination $\psi=\sum_{1 \leq j \leq r} c_{j}(\nu) \psi_{j}(\nu)$, with meromorphic functions $c_{j}: \mathfrak{a}_{F \mathrm{qC}}^{*} \rightarrow \mathbb{C}$. Using (10.4) we now deduce that

$$
g(\nu: x)=\sum_{j=1}^{r} c_{j}(\nu) p(\nu) K_{F}^{t}\left(\nu: x: y_{j}\right) v_{j}
$$

as an identity of meromorphic functions in the variable $\nu \in \mathfrak{a}_{F \mathrm{qc}}^{*}$. From Lemma 10.4 it follows that there exists a dense open subset $\Omega \subset \mathfrak{a}_{F \text { qC }}^{*}$ such that $\nu \mapsto g_{\nu}$ is regular on $\Omega$ and such that, for $\nu \in \Omega$, the inclusion (10.9) is valid. From $g \in \mathcal{E}_{F}^{\text {hyp }}(\mathrm{X}: \tau)$ it follows that there exists a finite subset $Y \subset{ }^{*} \mathfrak{a}_{F \mathrm{qC}}^{*}$ such that $g \in C_{F, Y}^{\text {ep,hyp }}\left(\mathrm{X}_{+}: \tau\right)$. By the same argument as at the end of the proof of Lemma 10.4 we now conclude that the inclusion (10.9) is valid for every $\nu \in \operatorname{reg} g$.

Corollary 10.6 Let notation be as in Theorem 10.5. Then, for each $\nu \in$ $i \mathfrak{a}_{F q}^{*} \cap \operatorname{reg} g$,

$$
g_{\nu} \in \mathcal{A}_{\text {temp }}(\mathrm{X}: \tau) \text {. }
$$

Proof: Let $\nu \in i \mathfrak{a}_{F \mathrm{q}}^{*} \cap \operatorname{reg} g$. Then from (10.9) it follows that every $\left(P_{0}, v\right)$ exponent of $g_{\nu}$ is of the form $\xi=s(\nu+\eta)-\rho-\mu$, with $s \in W^{F}, \eta \in \Lambda(F)$ and $\mu \in \mathbb{N} \Delta$. Now $\Lambda(F) \subset-\mathbb{R}_{+} F$, hence $s \eta \in-\mathbb{R}_{+} \Sigma^{+}$. It follows that $\operatorname{Re} \xi+\rho=$ $s \eta-\mu \in-\mathbb{R}_{+} \Delta$, hence $\operatorname{Re} \xi+\rho \leq 0$ on $A_{\mathrm{q}}^{+}\left(P_{0}\right)$. In view of Lemma 10.2 this implies that $g_{\nu} \in \mathcal{A}_{\text {temp }}(\mathrm{X}: \tau)$.

\section{Initial uniform estimates}

In this section we shall derive estimates for the generalized Eisenstein integrals $E_{F}^{\circ}(\nu)$, with uniformity in the parameter $\nu \in \mathfrak{a}_{F \mathrm{qc}}^{*}$, from similar estimates for the normalized Eisenstein integral $E^{\circ}(\lambda)=E^{\circ}\left(P_{0}: \lambda\right)$. The idea is that estimates of the latter survive the application of certain Laurent functionals.

We start with an investigation of the type of estimates involved. For $Q \in \mathcal{P}_{\sigma}$ and $R \in \mathbb{R}$, we define

$$
\mathfrak{a}_{Q \mathrm{q}}^{*}(Q, R):=\left\{\nu \in \mathfrak{a}_{Q \mathrm{qC}}^{*} \mid \operatorname{Re}\langle\nu, \alpha\rangle<R, \quad \forall \alpha \in \Sigma_{r}(Q)\right\} .
$$

The closure of this set is denoted by $\overline{\mathfrak{a}}_{Q q}^{*}(Q, R)$. It is readily seen to consist of all elements $\nu \in \mathfrak{a}_{Q \mathrm{qC}}^{*}$ with $\operatorname{Re}\langle\nu, \alpha\rangle \leq R$ for all $\alpha \in \Sigma_{r}(Q)$.

In the following lemma we assume that $S$ is a finite subset of $\mathfrak{a}_{Q \mathrm{q}}^{*} \backslash\{0\}$ and we use the notation of Section 4 
Lemma 11.1 Let $R \in \mathbb{R}, p \in \Pi_{S}\left(\mathfrak{a}_{Q \mathrm{q}}^{*}\right), u \in S\left(\mathfrak{a}_{Q \mathrm{q}}^{*}\right)$ and $n \in \mathbb{N}$. Then for every real number $R_{-}<R$ and every $\delta>0$ there exists a constant $C>0$ with the following property.

Assume that $V$ is any complete locally convex space, $s$ a continuous seminorm on $V$ and $b>0$ a constant. Moreover, let $f: \mathfrak{a}_{Q \mathrm{q}}^{*}(Q, R) \rightarrow V$ be a holomorphic function satisfying the estimate

$$
s(p(\nu) f(\nu)) \leq(1+|\nu|)^{n} e^{b|\operatorname{Re} \nu|},
$$

for all $\nu \in \mathfrak{a}_{Q \mathrm{q}}^{*}(Q, R)$. Then

$$
s(u f(\nu)) \leq C(1+|\nu|)^{n} e^{b \delta} e^{b|\operatorname{Re} \nu|},
$$

for all $\nu \in \mathfrak{a}_{Q \mathrm{q}}^{*}\left(Q, R_{-}\right)$.

Proof: It suffices to prove this on the one hand for $u=1$ and $p$ arbitrary and on the other hand for $p=1$ and $u$ arbitrary. In the first case the proof is essentially the same as that of Lemma $6.1 \mathrm{in} \mathrm{[5}$, which is based on an application of Cauchy's integral formula.

In the second case the proof relies on a straightforward application of Cauchy's integral formula.

Let $\mathcal{H}$ be a $\Sigma$-configuration in $\mathfrak{a}_{\mathrm{qC}}^{*}$. For $Y \subset{ }^{*} \mathfrak{a}_{Q \mathrm{qC}}^{*}$ a finite subset, we define the $\Sigma_{r}(Q)$-configuration $\mathcal{H}_{Q}(Y)=\mathcal{H}_{\mathfrak{a}_{Q \mathrm{qC}}^{*}}(Y)$ in $\mathfrak{a}_{Q \mathrm{qC}}^{*}$ as in (4.6), with $L=\mathfrak{a}_{Q \mathrm{qC}}^{*}$ and $S=Y$, see also 14], text preceding Cor. 11.6. Thus, for $\nu \in \mathfrak{a}_{Q \mathrm{qc}}^{*}$, we have

$$
\nu \in \mathfrak{a}_{Q \mathrm{qC}}^{*} \backslash \cup \mathcal{H}_{Q}(Y) \Longleftrightarrow\left\{\forall \lambda \in Y \forall H \in \mathcal{H}: \quad \lambda+\nu \in H \Rightarrow \lambda+\mathfrak{a}_{Q \mathrm{qC}}^{*} \subset H\right\} .
$$

Let now $\mathcal{L} \in \mathcal{M}\left({ }^{*} \mathfrak{a}_{Q \mathrm{qC}}^{*}, \Sigma_{Q}\right)_{\text {laur }}^{*}$ have support contained in the finite subset $Y$ of ${ }^{*} \mathfrak{a}_{Q \mathrm{qc}}^{*}$. For any locally convex space $V$ we have an associated continuous linear operator $\mathcal{L}_{*}$ as in (4.7). The following result expresses the continuity with uniformity in the space $V$.

Lemma 11.2 Let $\mathcal{H}, Y, \mathcal{L}$ be as above, and let $d: \mathcal{H} \rightarrow \mathbb{N}$ be a map. Then there exists a map $d^{\prime}: \mathcal{H}_{Q}(Y) \rightarrow \mathbb{N}$ with the following property. For any locally convex space $V$, the prescription

$$
\mathcal{L}_{*} f(\nu)=\mathcal{L}[f(\cdot+\nu)]
$$

defines a continuous linear operator

$$
\mathcal{L}_{*}: \mathcal{M}\left(\mathfrak{a}_{\mathrm{qC}}^{*}, \mathcal{H}, d, V\right) \rightarrow \mathcal{M}\left(\mathfrak{a}_{Q \mathrm{qC}}^{*}, \mathcal{H}_{Q}(Y), d^{\prime}, V\right) .
$$

Proof: This is Cor. 11.6 of 14 .

A real $\Sigma_{r}(Q)$-configuration $\mathcal{H}^{\prime}$ in $\mathfrak{a}_{Q \mathrm{qC}}^{*}$ consists of hyperplanes of the form

$$
H_{\alpha, s}:=\left\{\nu \in \mathfrak{a}_{Q \mathrm{qC}}^{*} \mid\langle\alpha, \nu\rangle=s\right\},
$$

with $\alpha \in \Sigma_{r}(Q)$ and $s \in \mathbb{R}$. The configuration $\mathcal{H}^{\prime}$ is called $Q$-bounded if there exists a constant $s_{0} \in \mathbb{R}$ such that $H_{\alpha, s} \in \mathcal{H}^{\prime} \Rightarrow s \geq s_{0}$, for all $\alpha \in \Sigma_{r}(Q), s \in \mathbb{R}$. See 11], text before Lemma 3.1, for the similar notion for $Q$ minimal. 
Lemma 11.3 Let $Q \in \mathcal{P}_{\sigma}, P \in \mathcal{P}_{\sigma}^{\min }$ and $P \subset Q$. Let $Y \subset{ }^{*} \mathfrak{a}_{Q q}^{*}$ be a finite subset.

(a) If $\mathcal{H}$ is a P-bounded real $\Sigma$-configuration in $\mathfrak{a}_{\mathrm{qc}}^{*}$, then $\mathcal{H}_{Q}(Y)$ is a $Q$ bounded real $\Sigma_{r}(Q)$-configuration in $\mathfrak{a}_{Q \mathrm{qC}}^{*}$.

(b) If $\mathcal{H}^{\prime}$ is a $Q$-bounded real $\Sigma_{r}(Q)$-configuration in $\mathfrak{a}_{Q \mathrm{qC}}^{*}$, then for every $R \in \mathbb{R}$ the collection $\left\{H \in \mathcal{H}^{\prime} \mid H \cap \overline{\mathfrak{a}}_{Q \mathrm{q}}^{*}(Q, R) \neq \emptyset\right\}$ is finite.

(c) If $\mathcal{H}^{\prime}$ is as in (b), then for every $R \in \mathbb{R}$ there exists a constant $R_{+}>R$ such that $H \cap \overline{\mathfrak{a}}_{Q \mathrm{q}}^{*}(Q, R) \neq \emptyset \Longleftrightarrow H \cap \mathfrak{a}_{Q \mathrm{q}}^{*}\left(Q, R_{+}\right) \neq \emptyset$, for every $H \in \mathcal{H}^{\prime}$.

Proof: There exists $t_{0} \in \mathbb{R}$ such that the hyperplanes in $\mathcal{H}$ are all of the form $H_{\beta, t}$, with $\beta \in \Sigma(P)$ and $t \in\left[t_{0}, \infty\left[\right.\right.$. Let $\eta \in Y$ and assume that $-\eta+H_{\beta, t}$ intersects $\mathfrak{a}_{Q \mathrm{qC}}^{*}$ in a proper hyperplane $H^{\prime}$. Then it follows that the restriction $\alpha=\left.\beta\right|_{\mathfrak{a}_{Q q}}$ is non-zero, hence belongs to $\Sigma_{r}(Q)$. Moreover, $H^{\prime}=H_{\alpha, s}$ with $s=t-\langle\beta, \eta\rangle$. Let $m$ be the maximum of the numbers $\langle\beta, \eta\rangle$, for $\beta \in \Sigma(P) \backslash \Sigma_{Q}$ and $\eta \in Y$ and put $s_{0}=t_{0}-m$. Then it follows that every hyperplane from $\mathcal{H}_{Q}(Y)$ is of the form $H_{\alpha, s}$, with $\alpha \in \Sigma_{r}(Q)$ and $s \geq s_{0}$. This establishes (a).

To prove (b), fix $\alpha \in \Sigma_{r}(Q)$ and put $I_{\alpha, R}=\left\{s \in \mathbb{R} \mid H_{\alpha, s} \in \mathcal{H}, H_{\alpha, s} \cap\right.$ $\left.\overline{\mathfrak{a}}_{Q \mathrm{q}}^{*}(Q, R) \neq \emptyset\right\}$, for every $R>0$. Then it suffices to show that $I_{\alpha, R}$ is finite. Since $\mathcal{H}$ is locally finite, the set $I_{\alpha, R}$ is discrete, and since $\mathcal{H}$ is $Q$-bounded, the set $I_{\alpha, R}$ is bounded from below. If $h \in H_{\alpha, s} \cap \overline{\mathfrak{a}}_{Q \mathrm{q}}^{*}(Q, R)$, then $s=\langle\alpha, h\rangle \leq R$. It follows that the set $I_{\alpha, R}$ is bounded from above by $R$. Hence, $I_{\alpha, R}$ is finite.

For (c) we observe that $R \leq R^{\prime} \Rightarrow I_{\alpha, R} \subset I_{\alpha, R^{\prime}}$. Fix $R^{\prime}>R$. Using that $I_{\alpha, R^{\prime}}$ is discrete, we see that we may choose $R_{+} \in$ ] $R, R^{\prime}$ [ sufficiently close to $R$ so that $I_{\alpha, R_{+}}=I_{\alpha, R}$ for all $\alpha \in \Sigma_{r}(Q)$. The constant $R_{+}$has the required property.

If $\mathcal{H}^{\prime}$ is a $Q$-bounded real $\Sigma_{r}(Q)$-configuration in $\mathfrak{a}_{Q \mathrm{qC}}^{*}$, and $d^{\prime}: \mathcal{H}^{\prime} \rightarrow \mathbb{N}$ a map, then, for $R \in \mathbb{R}$, we define the polynomial function $\pi_{Q, R, d^{\prime}}$ on $\mathfrak{a}_{Q \mathbf{q C}}^{*}$ in analogy with (4.3) by

$$
\pi_{Q, R, d^{\prime}}:=\prod_{H} l_{H}^{d^{\prime}(H)},
$$

where the product is taken over the collection of $H \in \mathcal{H}^{\prime}$ whose intersection with $\overline{\mathfrak{a}}_{Q \mathrm{q}}^{*}(Q, R)$ is non-empty; this collection is finite by Lemma 11.3 (b). It follows from Lemma 11.3 (c) that $\pi_{Q, R, d^{\prime}}=\pi_{Q, R_{+}, d^{\prime}}$, for $R_{+}>R$ sufficiently close to $R$.

Proposition 11.4 Let $Q \in \mathcal{P}_{\sigma}, P \in \mathcal{P}_{\sigma}^{\min }$ and $P \subset Q$. Let $Y \subset{ }^{*} \mathfrak{a}_{Q \mathrm{q}}^{*}$ be a finite subset and let $\mathcal{L} \in \mathcal{M}\left({ }^{*} \mathfrak{a}_{\text {Qq }}^{*}, \Sigma_{Q}\right)_{\text {laur }}^{*}$ be a Laurent functional with $\operatorname{supp} \mathcal{L} \subset$ $Y$. Let $\mathcal{H}$ be a P-bounded $\Sigma$-configuration in $\mathfrak{a}_{\mathrm{qC}}^{*}, d: \mathcal{H} \rightarrow \mathbb{N}$ a map, and let $d^{\prime}: \mathcal{H}_{Q}(Y) \rightarrow \mathbb{N}$ be associated with the above data as in Lemma 11.2 Let $M>$ $\max _{\eta \in Y}|\operatorname{Re} \eta|$ and assume that $R, R^{\prime} \in \mathbb{R}$ are constants with $Y+\mathfrak{a}_{Q \mathrm{q}}^{*}\left(Q, R^{\prime}\right) \subset$ $\mathfrak{a}_{\mathrm{q}}^{*}\left(P, R_{-}\right)$for some $R_{-}<R$.

There exists a constant $k \in \mathbb{N}$ and for every $n \in \mathbb{N}$ a constant $C>0$ with the following property. 
If $V$ is a complete locally convex space, $s$ a continuous seminorm on $V, b>0$ a positive constant and $\varphi$ a function in $\mathcal{M}\left(\mathfrak{a}_{\mathrm{qC}}^{*}, \mathcal{H}, d, V\right)$ satisfying the estimate

$$
s\left(\pi_{P, R, d}(\lambda) \varphi(\lambda)\right) \leq(1+|\lambda|)^{n} e^{b|\operatorname{Re} \lambda|},
$$

for all $\lambda \in \mathfrak{a}_{\mathrm{q}}^{*}(P, R)$, then the function $\mathcal{L}_{*} \varphi \in \mathcal{M}\left(\mathfrak{a}_{Q \mathrm{qC}}^{*}, \mathcal{H}_{Q}(Y), d^{\prime}, V\right)$ satisfies the estimate

$$
s\left(\pi_{Q, R^{\prime}, d^{\prime}}(\nu) \mathcal{L}_{*} \varphi(\nu)\right) \leq C(1+|\nu|)^{n+k} e^{b M} e^{b|\operatorname{Re} \nu|}
$$

for all $\nu \in \mathfrak{a}_{Q \mathrm{q}}^{*}\left(Q, R^{\prime}\right)$.

Proof: It suffices to prove this for the case that $\operatorname{supp} \mathcal{L}$ consists of a single point $\lambda_{0} \in Y$. Let $\mathcal{H}_{0}$ be the collection of $H \in \mathcal{H}$ containing $\lambda_{0}+\mathfrak{a}_{Q \mathrm{qc}}^{*}$. Then for every $H \in \mathcal{H}_{0}$ there is a unique indivisible root $\alpha_{H} \in \Sigma_{Q} \cap \Sigma(P)$ such that $H=$ $\lambda_{0}+\left(\alpha \frac{\perp}{H}\right)_{\mathbb{C}}$. We define the affine function $l_{H}: \mathfrak{a}_{\mathrm{qC}}^{*} \rightarrow \mathbb{C}$ by $l_{H}(\lambda)=\left\langle\lambda-\lambda_{0}, \alpha_{H}\right\rangle$. Then $H=l_{H}^{-1}(0)$. We define the polynomial function $q_{0}: \mathfrak{a}_{\mathrm{qC}}^{*} \rightarrow \mathbb{C}$ by

$$
q_{0}=\prod_{H \in \mathcal{H}_{0}} l_{H}^{d(H)}
$$

From the definition of the space of Laurent functionals in $\mathcal{M}\left({ }^{*} \mathfrak{a}_{Q \mathrm{qc}}^{*}, \Sigma_{Q}\right)_{\text {laur }}^{*}$ supported at $\lambda_{0}$, see 4 it follows that there exists a $u \in S\left({ }^{*} \mathfrak{a}_{Q \mathrm{q}}^{*}\right)$ such that on a function $f \in\left(q_{0} \mid{ }^{*} \mathfrak{a}_{Q \mathbf{q C}}^{*}\right)^{-1} \mathcal{O}_{\lambda_{0}}$ the action of the Laurent functional is given by $\mathcal{L} f=u\left(q_{0} \mid{ }_{\mathfrak{a}^{*}}^{*} f \mathrm{C}\right)\left(\lambda_{0}\right)$. It follows that for $\varphi \in \mathcal{M}\left(\mathfrak{a}_{\mathrm{qC}}^{*}, \mathcal{H}, d, V\right)$ and $\nu \in$ $\mathfrak{a}_{Q \mathrm{qC}}^{*} \backslash \cup \mathcal{H}_{Q}(Y)$

$$
\mathcal{L}_{*} \varphi(\nu)=\mathcal{L}(\varphi(\cdot+\nu))=u\left(q_{0}(\cdot) \varphi(\cdot+\nu)\right)\left(\lambda_{0}\right)=u\left(q_{0} \varphi\right)\left(\lambda_{0}+\nu\right) .
$$

As in the proof of [11, Lemma 1.2, we infer that there exist a polynomial function $\pi \in \Pi_{\Sigma_{r}(Q)}\left(\mathfrak{a}_{Q \mathrm{q}}^{*}\right)$ and finitely many $q_{j} \in P\left(\mathfrak{a}_{Q \mathrm{q}}^{*}\right)$ and $u_{j} \in S\left(\mathfrak{a}_{\mathrm{q}}^{*}\right)$, all independent of $V, s, b$ and $\varphi$, such that

$$
\pi(\nu) u\left(q_{0} \varphi\right)\left(\lambda_{0}+\nu\right)=\sum_{j} q_{j}(\nu) u_{j}\left(\pi_{P, R, d} \varphi\right)\left(\lambda_{0}+\nu\right),
$$

for $\nu \in \mathfrak{a}_{Q \mathrm{q}}^{*}\left(Q, R^{\prime}\right)$. Multiplying both sides of (11.1) with a suitable polynomial function we see that we may as well assume that $\pi=\pi_{0} \pi_{Q, R^{\prime}, d^{\prime}}$, for some $\pi_{0} \in \Pi_{\Sigma_{r}(Q)}\left(\mathfrak{a}_{Q q}^{*}\right)$. We obtain

$$
\pi_{0}(\nu) \psi(\nu)=\sum_{j} q_{j}(\nu) u_{j}\left(\pi_{P, R, d} \varphi\right)\left(\lambda_{0}+\nu\right),
$$

where we have written $\psi=\pi_{Q, R^{\prime}, d^{\prime}} \mathcal{L}_{*} \varphi$.

Let $k$ be the maximum of the degrees of the polynomials $q_{j}$. Then there exists a constant $D>0$, independent of $V, s, b$ and $\varphi$, such that for every $j$,

$$
\left|q_{j}(\nu)\right| \leq D(1+|\nu|)^{k}, \quad\left(\nu \in \mathfrak{a}_{Q \mathrm{qC}}^{*}\right) .
$$


Put $m=\max _{\eta \in Y}|\operatorname{Re} \eta|$ and fix $\delta>0$ such that $m+2 \delta<M$. We may select constants $R_{+}^{\prime}>R^{\prime}$ and $R_{-}<R$ such that $Y+\overline{\mathfrak{a}}_{Q \mathrm{q}}^{*}\left(Q, R_{+}^{\prime}\right) \subset \mathfrak{a}_{\mathrm{q}}^{*}\left(P, R_{-}\right)$. Adapting $R_{+}^{\prime}$ if necessary, we may in addition assume that

$$
\pi_{Q, R_{+}^{\prime}, d^{\prime}}=\pi_{Q, R^{\prime}, d^{\prime}}
$$

see the text preceding the proposition.

Let now $n \in \mathbb{N}$ and $b>0$, and assume that $\varphi$ satisfies the hypotheses of the proposition. It follows from Lemma 11.1 applied with $P$ in place of $Q$, that there exist constants $C_{j}>0$, independent of $V, s, b$ and $\varphi$, such that

$$
s\left(u_{j}\left(\pi_{P, R, d} \varphi\right)(\lambda)\right) \leq C_{j}(1+|\lambda|)^{n} e^{b \delta} e^{b|\operatorname{Re} \lambda|},
$$

for $\lambda \in \mathfrak{a}_{\mathrm{q}}^{*}\left(P, R_{-}\right)$.

Using the estimate $1+\left|\lambda_{0}+\nu\right| \leq\left(1+\left|\lambda_{0}\right|\right)(1+|\nu|)$ and combining (11.2), (11.3) and (11.5), we obtain

$$
s\left(\pi_{0}(\nu) \psi(\nu)\right) \leq C^{\prime}(1+|\nu|)^{n+k} e^{b \delta} e^{b(|\operatorname{Re} \nu|+m)}, \quad\left(\nu \in \mathfrak{a}_{Q \mathbf{q}}^{*}\left(Q, R_{+}^{\prime}\right)\right),
$$

with

$$
C^{\prime}=D\left(\sum_{j} C_{j}\right)\left(1+\left|\lambda_{0}\right|\right)^{n}
$$

From (11.4) we see that the function $\psi$ is holomorphic on $\mathfrak{a}_{Q q}^{*}\left(Q, R_{+}^{\prime}\right)$. We may therefore apply Lemma 11.1 with $\psi, \pi_{0}, C^{\prime}$ and $\left[C^{\prime} e^{b \delta+b m}\right]^{-1} s$ in place of $f, p, u$ and $s$, and with $R_{+}^{\prime}, R^{\prime}$ in place of $R, R_{-}$, respectively. Using that $m+2 \delta<M$, we obtain the desired estimate, with $C>0$ a constant that is independent of $V, s, b$ and $\varphi$.

In the rest of the section we shall apply the above results to Eisenstein integrals. We start with a suitable estimate for Eisenstein integrals associated with minimal $\sigma$-parabolic subgroups.

Lemma 11.5 Let $P \in \mathcal{P}_{\sigma}^{\min }$. Then there exists a $\bar{P}$-bounded real $\Sigma$-configuration $\mathcal{H}$ in $\mathfrak{a}_{\mathrm{qC}}^{*}$ and a map $d: \mathcal{H} \rightarrow \mathbb{N}$ such that the function $\lambda \mapsto E^{\circ}(P: \lambda)$ belongs to the space $\mathcal{M}\left(\mathfrak{a}_{\mathrm{qC}}^{*}, \mathcal{H}, d, C^{\infty}(\mathrm{X}) \otimes \operatorname{Hom}\left({ }^{\circ} \mathcal{C}, V_{\tau}\right)\right)$.

Let $R \in \mathbb{R}$ and let $p \in \Pi_{\Sigma}\left(\mathfrak{a}_{\mathrm{q}}^{*}\right)$ be a polynomial such that the function $\lambda \mapsto$ $p(\lambda) E^{\circ}(P: \lambda)$ is holomorphic on a neighborhood of $\overline{\mathfrak{a}}_{\mathrm{q}}^{*}(\bar{P}, R)$. Then there exists a constant $r>0$ and for every $u \in U(\mathfrak{g})$ constants $n \in \mathbb{N}$ and $C>0$ such that

$$
\left\|p(\lambda) E^{\circ}(P: \lambda: u ; x)\right\| \leq C(1+|\lambda|)^{n} e^{(r+|\operatorname{Re} \lambda|) l_{\mathrm{X}}(x)},
$$

for all $\lambda \in \overline{\mathfrak{a}}_{\mathrm{q}}^{*}(\bar{P}, R)$ and $x \in \mathrm{X}$. (See [10.1) for the definition of the function $l_{\mathrm{X}}$.)

Proof: First assume that $\tau=\tau_{\vartheta}$, defined as in [9], text after Eqn. (28), with $\vartheta \subset$ $\widehat{K}$ a finite subset. Then for $x \in A_{\mathrm{q}}$ the estimate (11.6) follows from [] , Corollary 16.2 and Proposition 10.3, combined with the fact that $E^{\circ}(P: \lambda)=E^{1}(\bar{P}: \lambda)$, see [9], Eqn. (52). In view of the decomposition $\mathrm{X}=K A_{\mathrm{q}}(e H)$, the estimate now follows for general $x \in \mathrm{X}$ by sphericality of the Eisenstein integral. Finally, for general $\tau$ the estimate follows by application of the 'functorial' dependence of the Eisenstein integral on $\tau$, see [9], Eqn. (32). 
We can now prove the following analogous result for the generalized Eisenstein integral.

Proposition 11.6 Let $F \subset \Delta, v \in{ }^{F} \mathcal{W}$ and let $t \in \mathrm{WT}(\Sigma)$ be a $W$-invariant even residue weight.

There exists a $\bar{P}_{F}$-bounded, real $\Sigma_{r}(F)$-hyperplane configuration $\mathcal{H}_{F}$ in $\mathfrak{a}_{F \mathbf{q C}}^{*}$ and a map $d_{F}: \mathcal{H}_{F} \rightarrow \mathbb{N}$ such that $\nu \mapsto E_{F}^{\circ}(\nu)$ belongs to the space

$$
\mathcal{M}\left(\mathfrak{a}_{Q \mathrm{qc}}^{*}, \mathcal{H}_{F}, d_{F}, C^{\infty}(\mathrm{X}) \otimes \operatorname{Hom}\left(\mathcal{A}_{F}^{*} t, V_{\tau}\right)\right) .
$$

Moreover, if $R^{\prime} \in \mathbb{R}$ and if $p$ is any polynomial in $\Pi_{\Sigma_{r}(F)}\left(\mathfrak{a}_{F \mathrm{q}}^{*}\right)$ such that $\nu \mapsto$ $p(\nu) E_{F, v}^{\circ}(\nu)$ is holomorphic on a neighborhood of $\overline{\mathfrak{a}}_{F q}^{*}\left(\bar{P}_{F}, R^{\prime}\right)$, then there exist a constant $r>0$ and for every $u \in U(\mathfrak{g})$ constants $n \in \mathbb{N}$ and $C>0$, such that

$$
\left\|p(\nu) E_{F, v}^{\circ}(\nu: u ; x)\right\| \leq C(1+|\nu|)^{n} e^{(r+|\operatorname{Re} \nu|) l_{\mathrm{X}}(x)},
$$

for all $\nu \in \overline{\mathfrak{a}}_{F \mathrm{q}}^{*}\left(\bar{P}_{F}, R^{\prime}\right)$ and $x \in \mathrm{X}$.

Remark 11.7 This result is a sharpening of the estimate given in 12, Lemma 10.8 .

Proof: According to Lemma 9.7 (b,c), the generalized Eisenstein integral may be expressed as

$$
E_{F, v}^{\circ}(\nu: x) \psi=\mathcal{L}_{*}\left[E^{\circ}\left(P_{0}: \cdot: x\right) \circ \mathrm{i}_{F, v}\right](\nu),
$$

with $\mathcal{L} \in \mathcal{M}\left(\mathfrak{a}_{F \mathrm{qC}}^{*}, \Sigma_{F}\right)_{\text {laur }}^{*} \otimes{ }^{\circ} \mathcal{C}_{F, v}$ a Laurent functional whose support is contained in a finite subset $Y \subset{ }^{*} \mathfrak{a}_{Q \mathrm{q}}^{*}$. Let $\mathcal{H}, d$ be associated with $P=P_{0}$ as in Lemma 11.5 Let $\mathcal{H}_{F}:=\mathcal{H}_{F}(Y)$ and $d_{F}:=d^{\prime}$ be associated with the data $P=P_{0}, Q=P_{F}, \mathcal{H}, d, \mathcal{L}$ as in Lemma 11.2 then $\mathcal{H}_{F}$ is a real $\bar{P}_{F}$-bounded $\Sigma_{r}(F)$-configuration, by Lemma 11.3 The first assertion of the proposition follows by application of Lemma 11.2

Fix $R \in \mathbb{R}$ such that $\mathfrak{a}_{F \mathrm{q}}^{*}\left(\bar{P}_{F}, R^{\prime}\right)+Y \subset \mathfrak{a}_{\mathrm{q}}^{*}\left(\bar{P}_{0}, R_{-}\right)$for some $R_{-}<R$. Let $r$ be the constant of Lemma 11.5 applied with $P_{0}, R$ and $\pi_{\bar{P}_{0}, R, d}$ in place of $P, R$ and $p$, respectively. Fix $u \in U(\mathfrak{g})$. Then according to Lemma 11.5 there exist constants $n_{0} \in \mathbb{N}$ and $C_{0}>0$, such that for all $x \in \mathrm{X}$,

$$
\left\|\pi_{\bar{P}_{0}, R, d}(\lambda) E^{\circ}\left(P_{0}: \lambda: u ; x\right) \circ \mathrm{i}_{F, v}\right\| \leq C_{0}(1+|\lambda|)^{n_{0}} e^{(r+|\operatorname{Re} \lambda|) l_{\mathrm{X}}(x)},
$$

for all $\lambda \in \overline{\mathfrak{a}}_{\mathrm{q}}^{*}\left(\bar{P}_{0}, R\right)$. Let $x \in \mathrm{X}$. We apply Proposition 11.4 to the function $\varphi=\varphi_{x}$ from $\mathcal{M}\left(\mathfrak{a}_{\mathrm{qC}}^{*}, \mathcal{H}, d, \operatorname{Hom}\left({ }^{\circ} \mathcal{C}_{F, v}, V_{\tau}\right)\right)$, given by

$$
\varphi_{x}=E^{\circ}\left(P_{0}: \cdot: u ; x\right) \circ \mathrm{i}_{F, v},
$$

with the constant $b=l_{\mathrm{X}}(x)$ and the seminorm $s=C_{0}^{-1} e^{-r b}\|\cdot\|$. Let $M>$ $\max _{\eta \in Y}|\operatorname{Re} \eta|$. Then we obtain the estimate

$$
\left\|\pi_{\bar{P}_{F}, R^{\prime}, d_{F}}(\nu) \mathcal{L}_{*}\left(\varphi_{x}\right)(\nu)\right\| \leq C_{1}(1+|\nu|)^{n_{0}+k} e^{(r+|\operatorname{Re} \nu|+M) l_{\mathrm{X}}(x)},
$$


for $\nu \in \mathfrak{a}_{F \mathrm{q}}^{*}\left(\bar{P}_{F}, R^{\prime}\right)$, with constants $k \in \mathbb{N}$ and $C_{1}>0$ that are independent of $x, \nu$. We now note that

$$
\begin{aligned}
\mathcal{L}_{*}\left(\varphi_{x}\right) & =\mathcal{L}_{*}\left(E^{\circ}\left(P_{0}: \cdot: u ; x\right) \circ \mathrm{i}_{F, v}\right)(\nu) \\
& =\mathcal{L}_{*}\left(E^{\circ}\left(P_{0}: \cdot\right) \circ \mathrm{i}_{F, v}\right)(\nu)(u ; x) \\
& =E_{F, v}^{\circ}(\nu: u ; x)
\end{aligned}
$$

as a meromorphic identity in $\nu \in \mathfrak{a}_{Q \mathrm{qc}}^{*}$. The second of the above identities involves the interchange of $u$ and $\mathcal{L}_{*}$, which is allowed by the continuity of $\mathcal{L}_{*}$, see Lemma 11.2 The third identity is obtained by application of (11.8). Thus, we obtain, for all $x \in \mathrm{X}$ and all $\nu \in \mathfrak{a}_{F \mathrm{q}}^{*}\left(\bar{P}_{F}, R^{\prime}\right)$, the estimate

$$
\left\|\pi_{\bar{P}_{F}, R^{\prime}, d_{F}}(\nu) E_{F, v}^{\circ}(\nu: u ; x)\right\| \leq C_{1}(1+|\nu|)^{n_{0}+k} e^{(r+|\operatorname{Re} \nu|+M) l_{\mathrm{X}}(x)} .
$$

This proves the result for the particular polynomial $p=\pi_{\bar{P}_{F}, R^{\prime}, d_{F}}$. For $p$ equal to a multiple of $\pi_{\bar{P}_{F}, R^{\prime}, d_{F}}$ the result now also follows, since any polynomial from $P_{d}\left(\mathfrak{a}_{F \mathrm{q}}^{*}\right), d \in \mathbb{N}$, can be estimated from above by a function of the form $C(1+|\nu|)^{d}$.

Let $p$ now be an arbitrary element of $\Pi_{\Sigma_{r}(F)}\left(\mathfrak{a}_{F \mathrm{q}}^{*}\right)$ satisfying the hypothesis. Fix $R_{+}^{\prime}>R^{\prime}$. Then an estimate of type (11.7) holds on $\mathfrak{a}_{F q}^{*}\left(\bar{P}_{F}, R_{+}^{\prime}\right)$ with $p \pi_{\bar{P}_{F}, R_{+}^{\prime}, d_{F}}$ in place of $p$. By application of Lemma 11.1 this implies an estimate of the form (11.7), with the required dependences of the constants.

\section{Symmetric pairs of residue type}

By $L_{\mathrm{d}}^{2}(X)$ we denote the discrete part of $L^{2}(\mathrm{X})$, i.e., the closed span in $L^{2}(\mathrm{X})$ of all the irreducible closed subspaces of $L^{2}(\mathrm{X})$. Accordingly we define

$$
L_{\mathrm{d}}^{2}(\mathrm{X}: \tau):=\left(L_{\mathrm{d}}^{2}(\mathrm{X}) \otimes V_{\tau}\right) \cap L^{2}(\mathrm{X}: \tau) .
$$

For the following definition we recall from Section 8 that the data $\left(G, H, K, \tau, \mathfrak{a}_{\mathrm{q}}, \Sigma^{+}\right)$ together with a $W$-invariant even residue weight $t \in \mathrm{WT}(\Sigma)$ determine the continuous linear operator $T_{\Delta}^{t}: C_{c}^{\infty}(\mathrm{X}: \tau) \rightarrow C^{\infty}(\mathrm{X}: \tau)$. If $G$ has a compact center modulo $H$, then this operator is given by the formula (8.9).

Definition 12.1 The reductive symmetric pair $(G, H)$ is said to be of residue type if the following conditions are fulfilled.

(a) The group $G$ has a compact center modulo $H$.

(b) For any choice of the data $\left(K, \mathfrak{a}_{\mathrm{q}}\right)$, the following requirement is fulfilled. For every finite dimensional unitary representation $\tau$ of $K$, every choice $\Sigma^{+}$of positive roots for $\Sigma$ and every $W$-invariant even residue weight $t \in \mathrm{WT}(\Sigma)$, the operator $T_{\Delta}^{t}: C_{c}^{\infty}(\mathrm{X}: \tau) \rightarrow C^{\infty}(\mathrm{X}: \tau)$ is the restriction of the orthogonal projection $L^{2}(\mathrm{X}: \tau) \rightarrow L_{\mathrm{d}}^{2}(\mathrm{X}: \tau)$. 
Remark 12.2 The above definition is given for technical reasons. Together with Definition [13.1] where the notion of residue type for a parabolic subgroup from $\mathcal{P}_{\sigma}$ will be introduced, it plays a role in a long chain of reasoning that will be used in an induction step in the proof of Theorem 21.2. (The induction goes by induction on the $\sigma$-split rank of $G$.) As part of the mentioned theorem it is asserted that in fact every pair $(G, H)$ with $G$ having compact center modulo $H$ and every group from $\mathcal{P}_{\sigma}$ is of residue type.

In the course of the chain of reasoning, many results will first be proved under the assumption that an involved parabolic subgroup from $\mathcal{P}_{\sigma}$ or an involved reductive symmetric pair $(G, H)$ is of residue type. Such results will be marked with (RT) after their declaration. The additional hypotheses will be clearly stated at the beginning of their proofs. Within the chain of reasoning, until Theorem 21.2 the results marked (RT) will only be used if these additional hypotheses are assumed to be fulfilled. The mentioned theorem implies that the additional hypotheses are in fact always fulfilled so that in the end the results marked (RT) are valid as stated.

Within the chain of reasoning, it also happens that definitions need extra hypotheses concerning residue type in order to be valid. These definitions will be marked (RT) as well. The extra hypotheses are stated in a subsequent remark. Within the chain of reasoning such definitions will only be used if the extra hypotheses are assumed to be fulfilled. In the end Theorem 21.2 implies that the extra hypotheses are always fulfilled, so that the definitions marked (RT) are valid as stated.

Remark 12.3 If $\mathfrak{a}_{\mathrm{q}}=\{0\}$, then $\mathrm{X}$ is compact and the operator $T_{\Delta}$ is understood to be the identity operator of $C_{c}^{\infty}(\mathrm{X}: \tau)$. Thus, conditions (a) and (b) of the definition are fulfilled and in this case $(G, H)$ is of residue type.

Remark 12.4 It follows from Lemma 8.10 that the notion of residue type is stable under isomorphisms of reductive symmetric pairs.

Remark 12.5 Condition (b) of the definition is valid as soon as a particular choice of the data $\left(K, \mathfrak{a}_{\mathrm{q}}\right)$ satisfies the mentioned requirement. Indeed, assume that $\left(K, \mathfrak{a}_{\mathrm{q}}\right)$ satisfies the requirement and let ' $K \subset G$ be a second $\sigma$-invariant maximal compact subgroup, $\mathfrak{g}=\mathfrak{k}^{\mathfrak{k}} \oplus^{\prime} \mathfrak{p}$ the associated Cartan decomposition, and $\mathfrak{a}_{\mathrm{q}} \subset \mathfrak{q} \cap ' \mathfrak{p}$ an associated maximal abelian subspace. Then there exists a (unique) $g \in \exp (\mathfrak{h} \cap \mathfrak{p})$ such that $g K g^{-1}=^{'} K$, see 38, p. 153. Now $\operatorname{Ad}(g) \mathfrak{a}_{\mathrm{q}}$ is maximal abelian in ' $\mathfrak{p} \cap \mathfrak{q}$, hence there exists an element $k \in{ }^{\prime} K_{e} \cap H_{e}$ such that $\operatorname{Ad}(k g) \mathfrak{a}_{\mathrm{q}}={ }^{\prime} \mathfrak{a}_{\mathrm{q}}$. Let $\varphi: G \rightarrow G$ be conjugation by $k g$, then $\varphi$ maps the data $\left(G, H, K, \mathfrak{a}_{\mathrm{q}}\right)$ onto $\left(G, H,{ }^{\prime} K, \mathfrak{a}_{\mathrm{q}}\right)$. In view of Lemma 8.10 it follows that the requirement in (b) for the pair $\left(K, \mathfrak{a}_{\mathrm{q}}\right)$ is equivalent to the similar requirement for the pair $\left(' K,{ }^{\prime} \mathfrak{a}_{\mathrm{q}}\right)$.

We recall from [5], $\S 17$, that the Schwartz space $\mathcal{C}(\mathrm{X}: \tau)$ is defined to be the space of functions $f \in C^{\infty}(\mathrm{X}: \tau)$ such that, for every $u \in U(\mathfrak{g})$ and $n \in \mathbb{N}$,

$$
s_{u, n}(f):=\sup _{x \in \mathrm{X}}\left(1+l_{\mathrm{X}}\right)^{n} \Theta(x)^{-1}\|u f(x)\|<\infty ;
$$


see also the beginning of $\S$ 10. The Schwartz space is equipped with the Fréchet topology determined by the seminorms $s_{u, n}$. By [3], Lemma 7.2, the operators from $\mathbb{D}(\mathrm{X})$ act on $\mathcal{C}(\mathrm{X}: \tau)$ by continuous linear endomorphisms. We define $\mathcal{A}_{2}(\mathrm{X}: \tau)$ to be the space of $\mathbb{D}(\mathrm{X})$-finite functions in $\mathcal{C}(\mathrm{X}: \tau)$.

Lemma 12.6 (RT) Let $G$ have compact center modulo $H$. Then, for every $W$-invariant even residue weight $t \in \mathrm{WT}(\Sigma)$,

$$
\mathcal{A}^{t}(\mathrm{X}: \tau)=L_{\mathrm{d}}^{2}(\mathrm{X}: \tau)=\mathcal{A}_{2}(\mathrm{X}: \tau)
$$

In particular, the space $\mathcal{A}_{2}(\mathrm{X}: \tau)$ is finite dimensional.

Remark 12.7 The fact that $L_{d}^{2}(\mathrm{X}: \tau)$ is finite dimensional is also an immediate consequence of the classification of the discrete series in 40. In the present paper it would not have been advantageous to use this known fact. Only the spectral properties of the discrete series as formulated in Theorem [16.1 are needed. The mentioned finite dimensionality naturally follows from the finite dimensionality of $\mathcal{A}^{t}(\mathrm{X}: \tau)$, by the nature of our argument.

Proof: We give the proof under the assumption that $(G, H)$ is of residue type, see Remark 12.2 It follows from Definition 12.1(b) that $T_{\Delta}^{t}\left(C_{c}^{\infty}(\mathrm{X}: \tau)\right)$ is dense in $L_{\mathrm{d}}^{2}(\mathrm{X}: \tau)$. By Lemma 9.1 it follows that $\mathcal{A}^{t}(\mathrm{X}: \tau)$ is dense in $L_{\mathrm{d}}^{2}(\mathrm{X}: \tau)$. By finite dimensionality of the first of these spaces, the first equality in (12.2) follows. In particular, it follows that the space $L_{\mathrm{d}}^{2}(\mathrm{X}: \tau)$ consists of smooth $\mathbb{D}(\mathrm{X})$-finite functions; by 3 , Thm. 7.3 with $p=2$ it is therefore contained in $\mathcal{A}_{2}(\mathrm{X}: \tau)$. Conversely, if $f \in \mathcal{A}_{2}(\mathrm{X}: \tau)$, then $f$ is $K$-finite and $\mathbb{D}(\mathrm{X})$-finite. Hence, by a well known result of Harish-Chandra its $(\mathfrak{g}, K)$-span in $\mathcal{C}\left(\mathrm{X}, V_{\tau}\right)$ is a $(\mathfrak{g}, K)$-module of finite length; see [1, p. 312, Thm. 12 and 42, p. 112, Thm. 4.2.1. The closure of this span in $L^{2}(\mathrm{X}) \otimes V_{\tau}$ is therefore a finite direct sum of irreducible representations. The mentioned closure contains $f$; hence, $f \in L_{d}^{2}(\mathrm{X}: \tau)$.

Assume that $G$ has compact center modulo $H$ and that $t \in \mathrm{WT}(\Sigma)$ is a $W$ invariant even residue weight. We recall that a choice of Hilbert structure on the space $\mathcal{A}^{t}(\mathrm{X}: \tau)$ uniquely determines an endomorphism $\alpha=\alpha^{t} \in \operatorname{End}\left(\mathcal{A}^{t}(\mathrm{X}: \tau)\right)$ such that (10.3) holds.

Lemma 12.8 (RT) Assume that $G$ has compact center modulo $H$ and let $t \in$ $\mathrm{WT}(\Sigma)$ be a $W$-invariant even residue weight.

(a) Assume that the space $\mathcal{A}^{t}(\mathrm{X}: \tau)$ is equipped with the restriction of the inner product from $L^{2}(\mathrm{X}) \otimes V_{\tau}$, see [12.2). Then the endomorphism $\alpha$, determined by (10.3), equals $|W|^{-1}$ times the identity operator of $\mathcal{A}^{t}(\mathrm{X}: \tau)$.

(b) The kernel $K_{\Delta}^{t}$ is independent of the residue weight $t$.

Proof: We give the proof under the assumption that $(G, H)$ is of residue type, see Remark 12.2 It follows from (12.2) that the real analytic $\tau \otimes 1$-spherical 
function e on $\mathrm{X}$ attains its values in $\operatorname{Hom}\left(L_{\mathrm{d}}^{2}(\mathrm{X}: \tau), V_{\tau}\right) \simeq V_{\tau} \otimes L_{\mathrm{d}}^{2}(\mathrm{X}: \tau)^{*}$. Hence, $\mathbf{e}^{*}: y \mapsto \mathbf{e}(y)^{*}$ is a real analytic $1 \otimes \tau^{*}$-spherical function on $\mathrm{X}$, with values in $\operatorname{Hom}\left(V_{\tau}, L_{\mathrm{d}}^{2}(\mathrm{X}: \tau)\right)$.

We define the continuous linear operator $P: C_{c}^{\infty}(\mathrm{X}: \tau) \rightarrow L_{\mathrm{d}}^{2}(\mathrm{X}: \tau)$ by

$$
P f=\int_{X} \mathbf{e}(y)^{*} f(y) d y .
$$

Then one readily verifies that $\langle P f \mid \psi\rangle=\langle f \mid \psi\rangle$ for every $\psi \in L_{\mathrm{d}}^{2}(\mathrm{X}: \tau)$. It follows that $P$ equals the restriction to $C_{c}^{\infty}(\mathrm{X}: \tau)$ of the orthogonal projection $L^{2}(\mathrm{X}: \tau) \rightarrow L_{\mathrm{d}}^{2}(\mathrm{X}: \tau)$. Hence, $P=T_{\Delta}^{t}$. Combining this with [8.9) we obtain that, for all $x \in \mathrm{X}$ and all $f \in C_{c}^{\infty}(\mathrm{X}: \tau)$,

$$
\begin{aligned}
|W| \int_{X} K_{\Delta}^{t}(x: y) f(y) d y & =T_{\Delta}^{t} f(x)=\mathbf{e}(x)(P f) \\
& =\int_{X} \mathbf{e}(x) \circ \mathbf{e}(y)^{*} f(y) d y .
\end{aligned}
$$

Since $\mathbf{e}(x) \circ \mathbf{e}(\cdot)^{*}$ and $K_{\Delta}^{t}(x: \cdot)$ are smooth and $\tau^{*} \otimes 1$-spherical functions on $\mathrm{X}$, with values in $\operatorname{End}\left(V_{\tau}\right)$, it follows from the above identities that $|W| K_{\Delta}^{t}(x: y)=$ $\mathbf{e}(x) \circ \mathbf{e}(y)^{*}$ for all $x, y \in \mathrm{X}$. This implies (10.3) with $\alpha$ equal to $|W|^{-1} I_{L_{\mathrm{d}}^{2}(\mathrm{X}: \tau)}$. Hence, (a) holds. Assertion (b) is now immediate.

\section{The normalized Eisenstein integral}

In this section we shall define the normalized Eisenstein integral, initially for the class of parabolic subgroups introduced in the following definition, see Remark 12.2

Definition 13.1 A parabolic subgroup $P \in \mathcal{P}_{\sigma}$ is said to be of residue type (relative to $H$ ) if for every $v \in N_{K}\left(\mathfrak{a}_{\mathrm{q}}\right)$ the pair $\left(M_{P}, M_{P} \cap v H v^{-1}\right)$ is of residue type. A subset $F \subset \Delta$ is said to be of residue type if the associated standard $\sigma$-parabolic subgroup $P_{F}$ is of residue type.

Remark 13.2 In view of Remark 12.4 it suffices to require the above condition for $v$ in a choice of representatives ${ }^{P} \mathcal{W} \subset N_{K}\left(\mathfrak{a}_{\mathrm{q}}\right)$ of $W_{P} \backslash W / W_{K \cap H}$.

Remark 13.3 We write ${ }^{\circ} G$ for $M_{G}$, the Langlands $M$-component of $G$ viewed as a parabolic subgroup. Thus, ${ }^{\circ} G$ equals the intersection of the kernels ker $|\chi|$, for $\chi: G \rightarrow \mathbb{C}^{*}$ a character. By the previous remark, $G$ (viewed as an element from $\left.\mathcal{P}_{\sigma}\right)$ is of residue type relative to $H$ if and only if the pair $\left({ }^{\circ} G,{ }^{\circ} G \cap H\right)$ is of residue type. If $G$ has compact center modulo $H$, then the pair $\left({ }^{\circ} G,{ }^{\circ} G \cap H\right)$ is of residue type if and only if $(G, H)$ is.

Definition 13.4 Two parabolic subgroups $P, Q \in \mathcal{P}_{\sigma}$ are said to be associated if their $\sigma$-split components $\mathfrak{a}_{P \mathrm{q}}$ and $\mathfrak{a}_{Q \mathrm{q}}$ are conjugate under $W$. The equivalence relation of associatedness is denoted by $\sim$. 
Lemma 13.5 Assume that $P \in \mathcal{P}_{\sigma}$ is of residue type. Then every $Q \in \mathcal{P}_{\sigma}$ with $Q \sim P$ has the same property.

Proof: If $Q \sim P$, there exists a $k \in N_{K}\left(\mathfrak{a}_{\mathrm{q}}\right)$ such that $\mathfrak{a}_{Q \mathrm{q}}=\operatorname{Ad}(k) \mathfrak{a}_{P \mathrm{q}}$. From this it follows that $M_{Q}=k M_{P} k^{-1}$. If $v \in N_{K}\left(\mathfrak{a}_{\mathrm{q}}\right)$, then $M_{Q} \cap v H v^{-1}$ equals $k\left(M_{P} \cap w H w^{-1}\right) k^{-1}$, with $w=k^{-1} v \in N_{K}\left(\mathfrak{a}_{\mathrm{q}}\right)$. The pair $\left(M_{P}, M_{P} \cap w H w^{-1}\right)$ is of residue type, and by Remark 12.4 we conclude that $\left(M_{Q}, M_{Q} \cap v H v^{-1}\right)$ is of residue type as well.

Let $P \in \mathcal{P}_{\sigma}$. We equip the space $\mathrm{X}_{P, v}$, for $v \in{ }^{P} \mathcal{W}$, with the invariant measure $d x_{P, v}$ specified at the end of Section 5 The space $\mathcal{A}_{2}\left(\mathrm{X}_{P, v}: \tau_{P}\right)$ is equipped with the inner product from $L^{2}\left(\mathrm{X}_{P, v}, V_{\tau}\right)$, for $v \in{ }^{P} \mathcal{W}$. Moreover, the space $\mathcal{A}_{2, P}=\mathcal{A}_{2, P, P \mathcal{W}}$ is defined to be the formal direct sum

$$
\mathcal{A}_{2, P}:=\bigoplus_{v \in P^{P} \mathcal{W}} \mathcal{A}_{2}\left(\mathrm{X}_{P, v}: \tau_{P}\right),
$$

equipped with the direct sum inner product. The space $\mathcal{A}_{2, P}$ is finite dimensional by Lemma 12.6 Application of this lemma requires $P$ to be of residue type, see Remark 12.2

We agree to denote by $\operatorname{pr}_{P, v}: \mathcal{A}_{2, P} \rightarrow \mathcal{A}_{2}\left(\mathrm{X}_{P, v}: \tau_{P}\right)$ the natural projection operator, for $v \in{ }^{P} \mathcal{W}$, and by $\mathrm{i}_{P, v}$ the associated natural embedding operator.

In the following we shall use the characterization of the generalized Eisenstein integral by its asymptotic behavior, see Theorem 9.3 to define an Eisenstein integral for arbitrary parabolic subgroups.

Proposition 13.6 (RT)

(a) Let $P \in \mathcal{P}_{\sigma}$. For every $\psi \in \mathcal{A}_{2, P}$ there exists a unique family $E^{\circ}(P: \psi) \in$ $\mathcal{E}_{P}^{\text {hyp }}(\mathrm{X}: \tau)$ with the following property. For all $\nu$ in a non-empty open subset of $\mathfrak{a}_{P \mathrm{qc}}^{*}$, each $v \in{ }^{P} \mathcal{W}$, every $X \in \mathfrak{a}_{P \mathrm{q}}$ and every $m \in \mathrm{X}_{P, v,+}$,

$$
q_{\nu-\rho_{P}}\left(P, v \mid E^{\circ}(P: \psi: \nu), X, m\right)=\psi_{v}(m) .
$$

(b) Let $F \subset \Delta$. Then, for every $W$-invariant even residue weight $t \in \mathrm{WT}(\Sigma)$, the space $\mathcal{A}_{F}^{*}$ equals $\mathcal{A}_{2, F}:=\mathcal{A}_{2, P_{F}}$; here we assume that in the definition of both spaces the same set ${ }^{F} \mathcal{W}$ has been used. Moreover, for every $\psi \in$ $\mathcal{A}_{2, F}$,

$$
E^{\circ}\left(P_{F}: \psi: \nu: x\right)=E_{F}^{\circ}(\nu: x) \psi,
$$

for all $x \in \mathrm{X}$ and generic $\nu \in \mathfrak{a}_{F \mathrm{qC}}^{*}$.

Proof: Here we prove (a) under the assumption that $P$ is of residue type and (b) under the assumption that $F$ is of residue type, see Remark 12.2.

Uniqueness follows from Theorem 6.11 Thus, it suffices to establish existence. We will first do this for $P$ of residue type and equal to a standard parabolic subgroup $P_{F}$, with $F \subset \Delta$. Let $t \in \mathrm{WT}(\Sigma)$ be any $W$-invariant even residue weight. Let ${ }^{*} t$ be the induced residue weight of $\Sigma_{F}$. Then it follows from 
Lemma 12.6 applied to the pair $\left(M_{F}, M_{F} \cap v H v^{-1}\right)$, that $\mathcal{A}^{*} t\left(\mathrm{X}_{F, v}: \tau_{F}\right)=$ $\mathcal{A}_{2}\left(\mathrm{X}_{F, v}: \tau_{F}\right)$. Moreover, if $\psi \in \mathcal{A}_{2}\left(\mathrm{X}_{F, v}: \tau_{F}\right)$, then $E^{\circ}\left(P_{F}: \psi\right):=E_{F}^{\circ}(\cdot) \psi$ satisfies the desired property, by Theorem 9.3

Now assume that $P$ is general and of residue type, let ${ }^{P} \mathcal{W} \subset N_{K}\left(\mathfrak{a}_{\mathrm{q}}\right)$ be a choice of representatives for $W_{P} \backslash W / W_{K \cap H}$ and let $\psi \in \mathcal{A}_{2, P}$. There exists a $u \in N_{K}\left(\mathfrak{a}_{\mathrm{q}}\right)$ such that $u^{-1} P u=P_{F}$, with $F \subset \Delta$. Moreover, $P_{F}$ is of residue type, by Lemma 13.5 The set ${ }^{F} \mathcal{W}=u^{-1}\left({ }^{P} \mathcal{W}\right)$ is a choice of representatives for $W_{F} \backslash W / W_{K \cap H}$ in $N_{K}\left(\mathfrak{a}_{\mathrm{q}}\right)$. For $v \in{ }^{P} \mathcal{W}$, let $\rho_{\tau, u}$ be the linear isomorphism from $C^{\infty}\left(\mathrm{X}_{F, u^{-1} v,+}: \tau_{F}\right)$ onto $C^{\infty}\left(\mathrm{X}_{P, v,+}: \tau_{P}\right)$, defined as in 14, Eqn. (3.24). We define the function $\psi^{\prime} \in \mathcal{A}_{2, F}$ by $\psi_{u^{-1} v}^{\prime}=\rho_{\tau, u}^{-1} \psi_{v}$, for $v \in{ }^{P} \mathcal{W}$. Define the meromorphic family $f: \mathfrak{a}_{P \mathrm{qC}}^{*} \rightarrow C^{\infty}(\mathrm{X}: \tau)$ by

$$
f_{\nu}(x)=E^{\circ}\left(P_{F}: \psi^{\prime}: u^{-1} \nu: x\right),
$$

for $x \in \mathrm{X}$ and generic $\nu \in \mathfrak{a}_{P \mathrm{qc}}^{*}$. By Theorem 9.3 and Lemma 6.12 the family $f$ belongs to $\mathcal{E}_{P}^{\text {hyp }}(\mathrm{X}: \tau)$. Moreover, it follows from [14, Lemma 3.6, that, for $v \in{ }^{P} \mathcal{W}$ and $\nu$ in a dense open subset of $\mathfrak{a}_{P \mathrm{qc}}^{*}$,

$$
\begin{aligned}
q_{\nu-\rho_{P}}\left(P, v \mid f_{\nu}\right) & =\left[\operatorname{Ad}\left(u^{-1}\right)^{*} \otimes \rho_{\tau, u}\right] q_{u^{-1}{ }_{\nu-\rho_{F}}}\left(P_{F}, u^{-1} v \mid f_{\nu}\right) \\
& =\rho_{\tau, u} \psi_{u^{-1} v}^{\prime}=\psi_{v} .
\end{aligned}
$$

This establishes the result with $E^{\circ}(P: \psi)=f$.

From the uniqueness assertion in Proposition 13.6 it follows that the meromorphic function $E^{\circ}(P: \psi): \mathfrak{a}_{P \mathrm{qc}}^{*} \rightarrow C^{\infty}(\mathrm{X}: \tau)$ depends linearly on $\psi$.

Definition 13.7 (RT) Let $P \in \mathcal{P}_{\sigma}$. For $\psi \in \mathcal{A}_{2, P}$, let $E^{\circ}(P: \psi)$ denote the unique family in $\mathcal{E}_{P}^{\text {hyp }}(\mathrm{X}: \tau)$ of Proposition 13.6 (a).

The meromorphic $C^{\infty}\left(\mathrm{X}, \operatorname{Hom}\left(\mathcal{A}_{2, P}, V_{\tau}\right)\right)$-valued function $E^{\circ}(P: \cdot)=E^{\circ}(\mathrm{X}: P: \cdot)$ on $\mathfrak{a}_{P \mathrm{qc}}^{*}$, defined by

$$
E^{\circ}(P: \nu: x) \psi=E^{\circ}(P: \psi: \nu: x)
$$

for $\psi \in \mathcal{A}_{2, P}, x \in \mathrm{X}$ and generic $\nu \in \mathfrak{a}_{Q \mathrm{qC}}^{*}$, is called the normalized Eisenstein integral associated with the parabolic subgroup $P$ (and the choice ${ }^{P} \mathcal{W}$ ).

The meromorphic $C^{\infty}\left(\mathrm{X}, \operatorname{Hom}\left(V_{\tau}, \mathcal{A}_{2, P}\right)\right)$-valued function $E^{*}(P: \cdot)$ on $\mathfrak{a}_{P \mathrm{qC}}^{*}$ defined by

$$
E^{*}(P: \nu: x)=E^{\circ}(P:-\bar{\nu}: x)^{*},
$$

for $\psi \in \mathcal{A}_{2, P}, x \in \mathrm{X}$, and generic $\nu \in \mathfrak{a}_{P \mathrm{qC}}^{*}$, is called the dual Eisenstein integral associated with $P$.

Remark 13.8 The above definition requires the validity of Proposition 13.6 which inside the chain of reasoning leading up to Theorem 21.2] requires $P$ to be of residue type, see Remark 12.2 
Remark 13.9 In the case of the group, the normalized Eisenstein integral defined above is essentially equal to the one defined by Harish-Chandra [29], $\S 6$, Thm. 6. This is seen as follows. Let ' $G$ be a real reductive group of Harish-Chandra's class, let $G={ }^{\prime} G \times{ }^{\prime} G$, let $\sigma: G \rightarrow G$ be the involution given by $\left(' x,{ }^{\prime} y\right) \mapsto\left(' y,{ }^{\prime} x\right)$ and let $H=G^{\sigma}$ be the diagonal subgroup. Then $\left({ }^{\prime} x,{ }^{\prime} y\right) \mapsto{ }^{\prime} x\left({ }^{\prime} y\right)^{-1}$ induces a $G$-diffeomorphism $\Phi: G / H \rightarrow{ }^{\prime} G$. Let ${ }^{\prime} \mathfrak{g}={ }^{\prime} \mathfrak{k} \oplus{ }^{\prime} \mathfrak{p}$ be a Cartan decomposition, ' $\theta$ the associated involution and put $\theta={ }^{\prime} \theta \times{ }^{\prime} \theta$. Then $\theta$ is a Cartan involution commuting with $\sigma$. Let ' $K$ and $K={ }^{\prime} K \times{ }^{\prime} K$ be the associated maximal compact subgroups of ' $G$ and $G$, respectively, and let $\left(\tau, V_{\tau}\right)$ be a finite dimensional unitary representation of $K$. Let $\tau_{1}, \tau_{2}$ be the unitary representations of ${ }^{\prime} K$ in $V_{\tau}$ defined by $\tau_{1}\left({ }^{\prime} k\right)=\tau\left({ }^{\prime} k, 1\right)$ and $\tau_{2}\left({ }^{\prime} k\right)=\left(1,{ }^{\prime} k\right)$. Let ' $\tau$ denote the pair of commuting representations $\left(\tau_{1}, \tau_{2}\right)$. Then pull-back by $\Phi$ induces a linear isomorphism $\Phi^{*}$ from the space $C^{\infty}\left({ }^{\prime} G:{ }^{\prime} \tau\right)$ of smooth ' $\tau$-spherical functions on ' $G$, onto $C^{\infty}(G / H: \tau)$.

Let $\mathfrak{a}_{\mathfrak{p}}$ be maximal abelian in $\mathfrak{p} \mathfrak{p}$, then $\mathfrak{a}_{\mathfrak{q}}:=\left\{(X,-X) \mid X \in \mathfrak{a}_{\mathfrak{p}}\right\}$ is maximal abelian in $\mathfrak{p} \cap \mathfrak{q}$. Let ' $P \subset{ }^{\prime} G$ be a parabolic subgroup containing $A_{\wedge_{\mathfrak{p}}}$. Then $P:={ }^{\prime} P \times^{\prime} \bar{P}$ belongs to $\mathcal{P}_{\sigma}$. Moreover, the map $(X, Y) \mapsto X-Y$ is surjective from $\mathfrak{a}_{P}=\mathfrak{a}_{P} \times \mathfrak{a}_{P}$ onto $\mathfrak{a}_{P}$ and induces a linear isomorphism from $\mathfrak{a}_{P \mathrm{q}}=\mathfrak{a}_{P} \cap \mathfrak{a}_{\mathrm{q}}$ onto $\mathfrak{a}_{P}$, mapping $\mathfrak{a}_{P \mathrm{q}}^{+}$onto $\mathfrak{a}_{P}^{+}$. The complexified adjoint map $\varphi^{*}$ is the linear isomorphism from $\mathfrak{a}_{P \mathbb{C}}^{*}$ onto $\mathfrak{a}_{P \mathrm{qC}}^{*}$ given by ${ }^{\prime} \nu \mapsto\left({ }^{\prime} \nu,-{ }^{\prime} \nu\right)$.

We observe that $M_{P}=M_{\wedge_{P}} \times M_{\wedge_{P}}$, so that $\mathrm{X}_{P}=M_{\wedge_{P}} \times M_{\wedge_{P}} / H \cap M_{P}$, which is $M_{P}$-diffeomorphic to $M_{\backslash_{P}}$ under the map $\Phi_{P}$ induced by restricting $\Phi$. It is readily seen that $\Phi_{P}^{*}$ restricts to a linear bijection from the finite dimensional space $L_{d}^{2}\left(M_{\wedge_{P}}:{ }^{\prime} \tau_{M_{\wedge_{P}}}\right)$ onto $L_{d}^{2}\left(\mathrm{X}_{P}: \tau_{P}\right)=\mathcal{A}_{2, P}$. Let $\psi \in L_{d}^{2}\left(\mathrm{X}_{P}: \tau_{P}\right)$ and consider the family $f: \mathfrak{a}_{\mathrm{qC}}^{*} \times G \rightarrow V_{\tau}$ defined by $f_{\nu}=\Phi^{*} E^{\circ}\left({ }^{\prime} P: \Phi_{P}^{*-1} \psi: \varphi^{*-1} \nu / i\right)$, where the normalized Eisenstein integral is Harish-Chandra's. By holomorphy of Harish-Chandra's unnormalized Eisenstein integral combined with meromorphy and the product structure of Harish-Chandra's $C$-function $C_{{ }_{P} \mid{ }^{\prime} P}\left(1:{ }^{\prime} \nu\right)$, it follows that the family $f_{\nu} \in C^{\infty}(\mathrm{X}: \tau)$ satisfies condition (a) of Definition 6.1 Via the subrepresentation theorem of [21, combined with induction by stages, Harish-Chandra's Eisenstein integral can be realized by matrix coefficients of the minimal principal series of ' $G$. Using this information it can be deduced that $f$ satisfies the remaining conditions (b), (c) of Definition 6.1 In fact, it is now readily checked that $f$ belongs to the space $C_{P}^{\text {hyp }}(\mathrm{X}: \tau)$, defined in Definition 6.6. Moreover, from the information on the constant term of Harish-Chandra's Eisenstein integral, see [29, §5, Thm. 5, it follows that $q_{\nu-\rho_{P}}\left(P, 1 \mid f_{\nu}, X, m\right)=\psi(m)$, in the notation of Proposition 13.6 for generic $\nu \in i \mathfrak{a}_{P \mathrm{q}}^{*}$. By meromorphy it now follows that the family $f$ satisfies the condition of Proposition 13.6 (a) (note that we may take ${ }^{P} \mathcal{W}=\{1\}$ here). Hence,

$$
\Phi^{*} E^{\circ}\left({ }^{\prime} P: \Phi_{P}^{*-1} \psi: \varphi^{*-1} \nu / i\right)=E^{\circ}(P: \nu) \psi \text {. }
$$

Remark 13.10 At the end of the sequel to this paper, [15], we will show that the normalized Eisenstein integral introduced above coincides (up to a change from $\nu$ to $-\nu$ ) with the one introduced by J. Carmona and P. Delorme in [19].

Remark 13.11 If $G$ has compact center modulo $H$, then $A_{G \mathrm{q}}=\{0\}$ and 
$\mathcal{A}_{2, G}=\mathcal{A}_{2}(G / H: \tau)$. In view of Lemma 12.6 it follows from Remark 9.4 that $E^{\circ}(G: 0: x)$ equals the evaluation map ev ${ }_{x}: \mathcal{A}_{2}(\mathrm{X}: \tau) \rightarrow V_{\tau}, \psi \mapsto \psi(x)$. Accordingly, $E^{*}(G: 0: x)=\mathrm{ev}_{x}^{*} \in \operatorname{Hom}\left(V_{\tau}, \mathcal{A}_{2}(\mathrm{X}: \tau)\right)$.

The following result describes the dependence of the normalized Eisenstein integral on a member $P$ of a class in $\mathcal{P}_{\sigma} / W$, as well as on the choice of ${ }^{P} \mathcal{W}$.

Lemma 13.12 (RT) Let $P \in \mathcal{P}_{\sigma}$, let $s \in W$, and let $Q:=s P s^{-1}$. Let ${ }^{P} \mathcal{W}$ and ${ }^{Q} \mathcal{W}$ be choices of representatives in $N_{K}\left(\mathfrak{a}_{\mathrm{q}}\right)$, for $W_{P} \backslash W / W_{K \cap H}$ and $W_{Q} \backslash W / W_{K \cap H}$, respectively. Then there exists a unique linear map $R_{P}(s): \mathcal{A}_{2, P} \rightarrow \mathcal{A}_{2, Q}$ such that

$$
E^{\circ}(Q: s \nu: x) \circ R_{P}(s)=E^{\circ}(P: \nu: x),
$$

for $x \in \mathrm{X}$ and generic $\nu \in \mathfrak{a}_{P \mathrm{qC}}^{*}$. The map $R_{P}(s)$ is bijective and unitary.

Proof: We give the proof under the assumption that $P$ is of residue type, see Remark 12.2 Left multiplication by $s$ induces a bijective map $W_{P} \backslash W / W_{K \cap H} \rightarrow$ $W_{Q} \backslash W / W_{K \cap H}$. Via the natural bijections ${ }^{P} \mathcal{W} \rightarrow W_{P} \backslash W / W_{K \cap H}$ and ${ }^{Q} \mathcal{W} \rightarrow$ $W_{Q} \backslash W / W_{K \cap H}$ we transfer the induced map to a bijection $\bar{s}:{ }^{P} \mathcal{W} \rightarrow{ }^{Q} \mathcal{W}$.

Let $\psi \in \mathcal{A}_{2, P}$ and define the meromorphic family $f: \mathfrak{a}_{Q \mathrm{qC}}^{*} \rightarrow C^{\infty}(\mathrm{X}: \tau)$ by

$$
f_{\lambda}(x)=E^{\circ}\left(P: s^{-1} \lambda: x\right) \psi,
$$

for $x \in \mathrm{X}$ and generic $\lambda \in \mathfrak{a}_{Q \mathrm{qc}}^{*}$. Then $f \in \mathcal{E}_{Q}^{\text {hyp }}(\mathrm{X}: \tau)$, by Lemma 6.12 Select $v \in{ }^{P} \mathcal{W}$. We may select a representative $u_{s}$ in $N_{K}\left(\mathfrak{a}_{\mathrm{q}}\right)$ of a Weyl group element from $s W_{P}$ such that $u_{s} v=\bar{s}(v) w_{s}$ for some $w_{s} \in N_{K \cap H}\left(\mathfrak{a}_{\mathrm{q}}\right)$. Note that $\mathrm{X}_{Q, u_{s} v}=$ $\mathrm{X}_{Q, \bar{s}(v)}$. Hence, we may define the bijective linear map

$$
\rho_{\tau, u_{s}}: \mathcal{A}_{2}\left(\mathrm{X}_{P, v}: \tau_{P}\right) \rightarrow \mathcal{A}_{2}\left(\mathrm{X}_{Q, \bar{s}(v)}: \tau_{Q}\right)
$$

as in 14], Eqn. (3.24). This map is unitary in view of the choice of invariant measures on $\mathrm{X}_{P, v}$ and $\mathrm{X}_{Q, \bar{s}(v)}$, specified at the end of Section [5]

It follows from [14, Lemmas 3.7 and 3.6, that

$$
\begin{aligned}
q_{\lambda-\rho_{Q}}\left(Q, \bar{s}(v) \mid f_{\lambda}\right) & =q_{\lambda-\rho_{Q}}\left(Q, u_{s} v \mid f_{\lambda}\right) \\
& =\left[\operatorname{Ad}\left(u_{s}^{-1}\right)^{*} \otimes \rho_{\tau, u_{s}}\right] q_{s^{-1} \lambda-\rho_{P}}\left(P, v \mid f_{\lambda}\right) \\
& =\rho_{\tau, u_{s}} \psi_{v},
\end{aligned}
$$

for generic $\lambda \in \mathfrak{a}_{Q \mathbf{q C}}^{*}$. Hence, by Definition 13.7

$$
f_{\lambda}(x)=E^{\circ}(Q: \lambda: x) \psi^{\prime},
$$

with $\psi_{\bar{s}(v)}^{\prime}=\rho_{\tau, u_{s}} \psi_{v}$ for $v \in{ }^{P} \mathcal{W}$. We define the bijective linear map $R_{P}(s): \mathcal{A}_{2, P} \rightarrow$ $\mathcal{A}_{2, Q}$ by

$$
\left(R_{P}(s) \psi\right)_{\bar{s}(v)}=\rho_{\tau, u_{s}} \psi_{v} .
$$

Then $\psi^{\prime}=R_{P}(s) \psi$ and (13.2) follows from (13.3) and (13.5) by substituting $s \nu$ for $\lambda$. From the definition it follows that $R_{P}(s)$ is unitary. 
To establish uniqueness, let $R_{P}(s): \mathcal{A}_{2, P} \rightarrow \mathcal{A}_{2, Q}$ be a linear map. Let $\psi \in$ $\mathcal{A}_{2, P}$, define $f$ as above, and define the meromorphic family $g: \mathfrak{a}_{Q \mathrm{qC}}^{*} \rightarrow C^{\infty}(\mathrm{X}: \tau)$ by $g_{\lambda}=E^{\circ}(Q: \lambda: \cdot) R_{P}(s) \psi$. Then

$$
q_{\lambda-\rho_{Q}}\left(Q, \bar{s}(v) \mid g_{\lambda}\right)=\left(R_{P}(s) \psi\right)_{\bar{s}(v)} .
$$

Now assume that (13.2) holds. Then $g=f$; combining (13.4) and (13.7) we obtain (13.6).

Let $P \in \mathcal{P}_{\sigma}$. Then for all $x, y \in \mathrm{X}$, the meromorphic $\operatorname{End}\left(V_{\tau}\right)$-valued function on $\mathfrak{a}_{P \mathrm{qC}}^{*}$ given by

$$
\nu \mapsto E^{\circ}(P: \nu: x) E^{*}(P: \nu: y)
$$

depends a priori on the choice of the set ${ }^{P} \mathcal{W}$.

Corollary 13.13 (RT) Let $P \in \mathcal{P}_{\sigma}$. Then for every $x, y \in \mathrm{X}$ the function [13.8) is independent of the particular choice of ${ }^{P} \mathcal{W}$.

Proof: Here we assume that $P$ is of residue type, see Remark 12.2 The result then follows from application of Lemma 13.12 with $s=1$.

Proposition 13.14 (RT) Let $P \in \mathcal{P}_{\sigma}$. There exists a $\bar{P}$-bounded, real $\Sigma_{r}(P)$ hyperplane configuration $\mathcal{H}=\mathcal{H}_{P}$ in $\mathfrak{a}_{P \mathrm{qC}}^{*}$ and a map $d=d_{P}: \mathcal{H} \rightarrow \mathbb{N}$ such that $\nu \mapsto E^{\circ}(P: \nu)$ belongs to the space

$$
\mathcal{M}\left(\mathfrak{a}_{P \mathrm{qC}}^{*}, \mathcal{H}, d, C^{\infty}(\mathrm{X}) \otimes \operatorname{Hom}\left(\mathcal{A}_{2, P}, V_{\tau}\right)\right) .
$$

Moreover, if $R \in \mathbb{R}$ and if $p$ is any polynomial in $\Pi_{\Sigma_{r}(P)}\left(\mathfrak{a}_{P \mathrm{q}}^{*}\right)$ such that $\nu \mapsto$ $p(\nu) E^{\circ}(P: \nu)$ is holomorphic on a neighborhood $\overline{\mathfrak{a}}_{P \mathrm{q}}^{*}(\bar{P}, R)$, then there exist a constant $r>0$ and for every $u \in U(\mathfrak{g})$ constants $n \in \mathbb{N}$ and $C>0$, such that

$$
\left\|p(\nu) E^{\circ}(P: \nu: u ; x)\right\| \leq C(1+|\nu|)^{n} e^{(r+|\operatorname{Re} \nu|) l_{\mathrm{x}}(x)},
$$

for all $\nu \in \overline{\mathfrak{a}}_{P \mathrm{q}}^{*}(\bar{P}, R)$ and $x \in \mathrm{X}$.

Proof: Here we prove the result for $P$ of residue type, see Remark 12.2

We first assume that $P=P_{F}$ with $F \subset \Delta$ of residue type. In this case, $E^{\circ}(P: \nu)=E_{F}^{\circ}(\nu)$, by Proposition 13.6 Hence, the result follows from Proposition 11.6 by summation over ${ }^{F} \mathcal{W}$, see (9.14) and (9.2).

Next, let $P \in \mathcal{P}_{\sigma}$ be a general parabolic subgroup of residue type. There exists a $s \in W$ such that $s P s^{-1}=P_{F}$, by Lemma [3.6 (c). Since $P$ is of residue type, $P_{F}$ is of residue type as well, see Lemma 13.5 By Lemma 13.12 and Proposition 13.6 there exists a unitary map $R_{P}(s): \mathcal{A}_{2, P} \rightarrow \mathcal{A}_{F, 2}$ such that

$$
E^{\circ}\left(P_{F}: s \nu: x\right) \circ R_{P}(s)=E^{\circ}(P: \nu: x)
$$

for all $x$ and generic $\nu \in \mathfrak{a}_{P \mathrm{qC}}^{*}$. The result now follows by application of the first part of the proof. 
The following result limits the exponents of the normalized Eisenstein integral along a minimal parabolic subgroup. To formulate it we need the following notation. Let $Q \in \mathcal{P}_{\sigma}$ and let $P \in \mathcal{P}_{\sigma}^{\text {min }}$ be such that $P \subset Q$. We put

$$
\begin{aligned}
\Delta_{Q}(P) & :=\left\{\alpha \in \Delta(P)|\alpha|_{\mathfrak{a}_{Q \mathrm{q}}}=0\right\} \\
W^{P \mid Q} & :=\left\{t \in W \mid t\left(\Delta_{Q}(P)\right) \subset \Sigma(P)\right\} .
\end{aligned}
$$

Let $s \in W$ be the unique element such that $P=s P_{0} s^{-1}$. Then $s^{-1} Q s$ contains $P_{0}$ hence equals $P_{F}$ for some subset $F \subset \Delta$; note that $F$ is uniquely determined by $Q$ in view of Lemma 3.6 We define

$$
\Lambda(P \mid Q):=s \Lambda(F),
$$

where $\Lambda(F)$ is the finite subset of $-\mathbb{R}_{+} F$ introduced in (8.7). We note that $\Lambda(P \mid Q)$ is a finite subset of $-\mathbb{R}_{+} \Delta_{Q}(P)$.

Proposition 13.15 (RT) Let $Q \in \mathcal{P}_{\sigma}$ and let $P \in \mathcal{P}_{\sigma}^{\min }$ be contained in $Q$. Let $\psi \in \mathcal{A}_{2, Q}$ and $q \in \Pi_{\Sigma_{r}(Q)}\left(\mathfrak{a}_{Q q}^{*}\right)$. Then $f:(\nu, x) \mapsto q(\nu) E^{\circ}(Q: \nu: x) \psi$ defines a family in $\mathcal{E}_{Q}^{\text {hyp }}(\mathrm{X}: \tau)$. Moreover, for each $v \in N_{K}\left(\mathfrak{a}_{\mathrm{q}}\right)$ and every $\nu \in \operatorname{reg} f$,

$$
\operatorname{Exp}\left(P, v \mid f_{\nu}\right) \subset W^{P \mid Q}(\nu+\Lambda(P \mid Q))-\rho_{P}-\mathbb{N} \Delta(P) .
$$

In particular, $f_{\nu} \in \mathcal{A}_{\text {temp }}(\mathrm{X}: \tau)$, for every $\nu \in i \mathfrak{a}_{Q \mathrm{q}}^{*} \cap \operatorname{reg} f$.

Remark 13.16 For $P$ minimal, the assertion about temperedness is due to [5], Thm. 19.2, in view of [9], Eqn. (52). For general $P$ the assertion about temperedness is due to [22, Thm. 1, in view of Remark 13.10]

Proof: We give the proof under the assumption that $Q$ is of residue type, see Remark 12.2 Let $s \in W$ and $F \subset \Delta$ be as in the text preceding the corollary. Let the polynomial function $p: \mathfrak{a}_{F \mathrm{qC}}^{*} \rightarrow \mathbb{C}$ be defined by $p(\nu)=q(s \nu)$. Then $p \in \Pi_{\Sigma_{r}(F)}\left(\mathfrak{a}_{F \mathrm{q}}^{*}\right)$. It follows from Lemma 13.12 with $P_{F}$ in place of $P$ that $f(\nu, x)=g\left(s^{-1} \nu, x\right)$, for $x \in \mathrm{X}$ and generic $\nu \in \mathfrak{a}_{Q \mathrm{qC}}^{*}$, where $g:(\lambda, x) \mapsto$ $p(\lambda) E_{F}^{\circ}(\lambda: x) R_{P_{F}}(s)^{-1} \psi$. By Theorem 10.5 the family $g$ belongs to $\mathcal{E}_{F}^{\text {hyp }}(\mathrm{X}: \tau)$. By Lemma 6.12 it follows that $f \in \mathcal{E}_{Q}^{\text {hyp }}(\mathrm{X}: \tau)$. Moreover, let $\nu \in \operatorname{reg} f$; then $s^{-1} \nu \in \operatorname{reg} g$ and by the last mentioned theorem it follows that, for every $u \in$ $N_{K}\left(\mathfrak{a}_{\mathrm{q}}\right)$,

$$
\begin{aligned}
\operatorname{Exp}\left(P_{0}, u \mid f_{\nu}\right) & =\operatorname{Exp}\left(P_{0}, u \mid g_{s^{-1}}\right) \\
& \subset W^{F}\left(s^{-1} \nu+\Lambda(F)\right)-\rho-\mathbb{N} \Delta .
\end{aligned}
$$

On the other hand, by [14, Lemma 3.6, it follows that, for $v \in N_{K}\left(\mathfrak{a}_{\mathrm{q}}\right)$,

$$
\operatorname{Exp}\left(P, v \mid f_{\nu}\right)=s \operatorname{Exp}\left(P_{0}, \bar{s}^{-1} v \mid f_{\nu}\right),
$$

where $\bar{s}$ is any representative of $s$ in $N_{K}\left(\mathfrak{a}_{\mathrm{q}}\right)$. We conclude that

$$
\operatorname{Exp}\left(P, v \mid f_{\nu}\right) \subset s W^{F} s^{-1}(\nu+s \Lambda(F))-\rho_{P}-\mathbb{N} \Delta(P) .
$$

Now $s \Lambda(F)=\Lambda(P \mid Q)$ by definition. Moreover, one readily verifies that $s W^{F} s^{-1}=$ $W^{P \mid Q}$. Hence, 13.10 follows. The final assertion follows from the similar assertion for $g$, which in turn follows by application of Corollary 10.6. 
In the theory of the constant term, we shall need the following result on the coefficients of the asymptotic expansions of the Eisenstein integral.

Lemma 13.17 (RT) Let $P \in \mathcal{P}_{\sigma}$ and let $\psi \in \mathcal{A}_{2, P}$. The family $f:(\nu, x) \mapsto$ $E^{\circ}(P: \nu: x) \psi$ belongs to $\mathcal{E}_{P, Y}^{\text {hyp }}(\mathrm{X}: \tau)$, for a suitable finite subset $Y \subset{ }^{*} \mathfrak{a}_{P \mathrm{q}}^{*}$. Moreover, the $\Sigma_{r}(P)$-configuration $\mathcal{H}_{f}$, defined as in the text before Definition 6.3, is real.

Let $k=\operatorname{deg}_{a} f$. Then for every $Q \in \mathcal{P}_{\sigma}, v \in N_{K}\left(\mathfrak{a}_{\mathrm{q}}\right)$, each $\sigma \in W / \sim_{Q \mid P}$ and all $\xi \in-\sigma \cdot Y+\mathbb{N} \Delta_{r}(Q)$,

$$
q_{\sigma, \xi}(Q, v \mid f) \in P_{k}\left(\mathfrak{a}_{Q \mathrm{q}}\right) \otimes \mathcal{M}\left(\mathfrak{a}_{P \mathrm{qC}}^{*}, \mathcal{H}_{f}, d_{f}, C^{\infty}\left(\mathrm{X}_{Q, v}: \tau_{Q}\right)\right) .
$$

Proof: We give the proof under the assumption that $P$ is of residue type, see Remark 12.2. In view of Lemma 3.6 (a), there exist $s \in W$ and $F \subset \Delta$ such that $P=s P_{F} s^{-1}$. In view of Lemma 13.12 with $P$ and $P_{F}$ in place of $Q$ and $P$, respectively, we may as well assume that $P=P_{F}$ for some $F \subset \Delta$. In this case we have $E^{\circ}(P: \nu)=E_{F}^{\circ}(\nu)$ by Proposition [13.6. Hence, the result follows from Lemma 9.8

Lemma 13.18 (RT) Let $F \subset \Delta$ and let $t \in \mathrm{WT}(\Sigma)$ be $a W$-invariant even residue weight. Then, for all $x, y \in \mathrm{X}$,

$$
K_{F}^{t}(\nu: x: y)=\left|W_{F}\right|^{-1} E^{\circ}\left(P_{F}: \nu: x\right) \circ E^{*}\left(P_{F}: \nu: y\right),
$$

as an identity of $\operatorname{End}\left(V_{\tau}\right)$-valued meromorphic functions in the variable $\nu \in$ $\mathfrak{a}_{F \mathbf{q C}}^{*} \cdot$

In particular, the function $K_{F}^{t}$ does not depend on the residue weight $t$, nor on the choice of ${ }^{F} \mathcal{W}$.

Proof: We give the proof under the assumption that $F$ is of residue type, see Remark 12.2 From Proposition13.6 (b) we recall that $\mathcal{A}_{F}^{* t}=\mathcal{A}_{2, F}$. Accordingly, we equip the space $\mathcal{A}_{F}^{*} t$ with the inner product described in the text preceding (13.1). As in the text preceding (10.4), this choice of inner product determines an endomorphism $\alpha_{F, v} \in \operatorname{End}\left(\mathcal{A}^{*} t\left(\mathrm{X}_{F, v}: \tau_{F}\right)\right)$, for each $v \in{ }^{F} \mathcal{W}$. The endomorphism $\alpha_{F, v}$ is the analogue for the space $\mathrm{X}_{F, v}$ of the endomorphism $\alpha$, described in (10.3). Thus, $\alpha_{F, v}=\left|W_{F}\right|^{-1} I$, by Lemma 12.8 Let $\alpha_{F} \in \operatorname{End}\left(\mathcal{A}_{F}^{*} t\right)$ be the direct sum of the $\alpha_{F, v}$, for $v \in{ }^{F} \mathcal{W}$. Then from (10.4) we obtain that

$$
K_{F}^{t}(\nu: x: y)=\left|W_{F}\right|^{-1} E_{F}^{\circ}(\nu: x) E_{F}^{\circ}(-\bar{\nu}: y)^{*},
$$

for all $x, y \in \mathrm{X}$ and generic $\nu \in \mathfrak{a}_{F \mathrm{qC}}^{*}$. Now use Definition 13.7 and Proposition 13.6 to conclude the validity of (13.12). It is now obvious that $K_{F}^{t}$ does not depend on $t$; it follows by application of Corollary 13.13 that it does not depend on ${ }^{F} \mathcal{W}$ either.

Remark 13.19 In view of Lemma 13.18 we agree to omit $t$ in the notation $K_{F}^{t}$. 
Definition 13.20 (RT) Let $P \in \mathcal{P}_{\sigma}$. We define the meromorphic function $K_{P}: \mathfrak{a}_{P \mathrm{qC}}^{*} \rightarrow C^{\infty}\left(\mathrm{X} \times \mathrm{X}, \operatorname{End}\left(V_{\tau}\right)\right)$ by

$$
K_{P}(\nu: x: y)=\left|W_{P}\right|^{-1} E^{\circ}(P: \nu: x) E^{*}(P: \nu: y),
$$

for $x, y \in \mathrm{X}$ and generic $\nu \in \mathfrak{a}_{P \mathrm{qc}}^{*}$.

In the chain of reasoning leading up to Theorem 21.2 this definition requires $P$ to be of residue type, since only then the Eisenstein integral is well-defined, see Remark 12.2

Remark 13.21 If $P=P_{F}$, for $F \subset \Delta$, then $K_{P}=K_{F}$, in view of Lemma 13.18

Lemma 13.22 (RT) Let $P \in \mathcal{P}_{\sigma}$. Then, for every $s \in W$ and all $x, y \in \mathrm{X}$,

$$
K_{P}(\nu: x: y)=K_{s P s^{-1}}(s \nu: x: y)
$$

as a meromorphic identity in $\nu \in \mathfrak{a}_{P \mathrm{qc}}^{*}$.

Proof: We give the proof under the assumption that $P$ is of residue type, see Remark 12.2 Put $Q=s P s^{-1}$. Since the inner product $c B$, specified in Section [5] is $W$-invariant, the normalized measures $d \mu_{P}$ and $d \mu_{Q}$ are $s$-conjugate. Moreover, since $W_{Q}=s W_{P} s^{-1}$, we have $\left|W_{Q}\right|=\left|W_{P}\right|$. The result now follows from combining Definition 13.20 and Lemma 13.12

Theorem 13.23 (RT) Let $P, Q \in \mathcal{P}_{\sigma}$ be associated parabolic subgroups. Then for every $s \in W\left(\mathfrak{a}_{Q \mathrm{q}} \mid \mathfrak{a}_{P \mathrm{q}}\right)$, and all $x, y \in \mathrm{X}$,

$$
K_{Q}(s \nu: x: y)=K_{P}(\nu: x: y),
$$

as an identity of $\operatorname{End}\left(V_{\tau}\right)$-valued meromorphic functions of the variable $\nu \in$ $\mathfrak{a}_{P \mathrm{qc}}^{*}$.

Proof: We give the proof under the assumption that $P$ and $Q$ are of residue type, see Remark 12.2 Since $P$ and $Q$ are associated, $\operatorname{dim} \mathfrak{a}_{Q q}=\operatorname{dim} \mathfrak{a}_{P \mathfrak{q}}$; hence, $s$ is a linear bijection from $\mathfrak{a}_{P \mathrm{q}}$ onto $\mathfrak{a}_{Q \mathrm{q}}$. From Corollary 3.5 it follows that there exists $w \in W$ such that $w\left(\mathfrak{a}_{P \mathrm{q}}\right)=\mathfrak{a}_{Q \mathrm{q}}$ and $\left.w\right|_{\mathfrak{a}_{P \mathrm{q}}}=s$.

Assume first that $P=P_{F}$ and $Q=P_{F^{\prime}}$, with $F, F^{\prime} \subset \Delta$. Then the result follows from 12, Lemma 6.2, since $K_{F}^{t}=K_{P_{F}}$ and $K_{F^{\prime}}^{t}=K_{P_{F^{\prime}}}$, for any $W$-invariant even residue weight $t$, see Remarks 13.19 and 13.21

Next assume that $P$ and $Q$ are arbitrary. Then the result follows by using Lemma 3.6 (a) and Lemma 13.22 


\section{Eigenvalues for the Eisenstein integral}

In this section we investigate the action of $\mathbb{D}(\mathrm{X})$ on the normalized Eisenstein integral.

Let $P \in \mathcal{P}_{\sigma}$. We define the algebra homomorphism $\mu_{P}: \mathbb{D}(\mathrm{X}) \rightarrow \mathbb{D}\left(\mathrm{X}_{1 P}\right)$ as in [5], text following (20). Here $\mathrm{X}_{1 P}:=\mathrm{X}_{1 P, e}=M_{1 P} / M_{1 P} \cap H$. Let $\mathfrak{b} \subset \mathfrak{q}$ be a $\theta$-stable Cartan subspace containing $\mathfrak{a}_{P \mathfrak{q}}$ and let $\gamma_{\mathfrak{b}}$ be the associated HarishChandra isomorphism from $\mathbb{D}(\mathrm{X})$ onto $I(\mathfrak{b})$. Let $W_{P}(\mathfrak{b})$ denote the centralizer of $\mathfrak{a}_{P \mathrm{q}}$ in $W(\mathfrak{b})$, and $I_{P}(\mathfrak{b})$ the ring of $W_{P}(\mathfrak{b})$-invariants in $S(\mathfrak{b})$. Moreover, let $\gamma_{\mathfrak{b}}^{\mathrm{X}_{1 P}}$ denote the associated Harish-Chandra isomorphism $\mathbb{D}\left(\mathrm{X}_{1 P}\right) \rightarrow I_{P}(\mathfrak{b})$. Then we recall from [5], Eqn. (21), that

$$
\gamma_{\mathfrak{b}}^{\mathrm{X}_{1 P}} \circ \mu_{P}=\gamma_{\mathfrak{b}}
$$

If $v \in N_{K}\left(\mathfrak{a}_{\mathrm{q}}\right)$, then following [14, text above Lemma 4.12, we define the algebra homomorphism $\mu_{P}^{v}: \mathbb{D}\left(G / v H v^{-1}\right) \rightarrow \mathbb{D}\left(\mathrm{X}_{1 P, v}\right)$ as $\mu_{P}$ for the triple $\left(G, v H v^{-1}, P\right)$ instead of $(G, H, P)$. Moreover, we define the algebra homomorphism $\mu_{P, v}: \mathbb{D}(\mathrm{X}) \rightarrow$ $\mathbb{D}\left(\mathrm{X}_{1 P, v}\right)$ by

$$
\mu_{P, v}=\mu_{P}^{v} \circ \operatorname{Ad}(v),
$$

where $\operatorname{Ad}(v)$ denotes the isomorphism $\mathbb{D}(\mathrm{X}) \rightarrow \mathbb{D}\left(G / v H v^{-1}\right)$ induced by the adjoint action by $v$. Since $A_{P \mathrm{q}}$ is central in $M_{1 P}$, it follows from (2.3) that

$$
\mathbb{D}\left(\mathrm{X}_{1 P, v}\right) \simeq \mathbb{D}\left(\mathrm{X}_{P, v}\right) \otimes S\left(\mathfrak{a}_{P q}\right)
$$

Accordingly, if $D \in \mathbb{D}(\mathrm{X})$, we shall write $\mu_{P, v}(D: \cdot)$ for $\mu_{P, v}(D)$, viewed as a $\mathbb{D}\left(\mathrm{X}_{P, v}\right)$-valued polynomial function on $\mathfrak{a}_{P \mathrm{qC}}^{*}$. If $D \in \mathbb{D}(\mathrm{X}), \nu \in \mathfrak{a}_{P \mathrm{qC}}^{*}$, and $v \in{ }^{P} \mathcal{W}$, then $\mu_{P, v}(D: \nu) \in \mathbb{D}\left(\mathrm{X}_{P, v}\right)$ acts on the space $\mathcal{A}_{2}\left(\mathrm{X}_{P, v}: \tau_{P}\right)$ (see the text preceding Lemma 12.6) by an endomorphism that we denote by $\underline{\mu}_{P, v}(D: \nu)$. The direct sum of these endomorphisms, for $v \in{ }^{P} \mathcal{W}$, is an endomorphism of the space $\mathcal{A}_{2, P}$, denoted $\underline{\mu}_{P}(D: \nu)$.

Lemma 14.1 (RT) Let $P \in \mathcal{P}_{\sigma}$. Then

$$
D E^{\circ}(P: \nu)=E^{\circ}(P: \nu) \underline{\mu}_{P}(D: \nu), \quad(D \in \mathbb{D}(\mathrm{X})) .
$$

Proof: We give the proof under the assumption that $P$ is of residue type, see Remark 12.2. Let $\psi \in \mathcal{A}_{2, P}$. Then the family $f: \mathfrak{a}_{P \mathrm{qC}}^{*} \times \mathrm{X} \rightarrow V_{\tau}$, defined by

$$
f(\nu, x)=E^{\circ}(P: \nu: x) \psi
$$

belongs to $\mathcal{E}_{P}^{\text {hyp }}(\mathrm{X}: \tau)$, by Proposition 13.6 Let $D \in \mathbb{D}(\mathrm{X})$. The family $D f:(\nu, x) \mapsto$ $D f_{\nu}(x)$ belongs to $\mathcal{E}_{P}^{\text {hyp }}(\mathrm{X}: \tau)$ as well, by Definition [6.6] and [14, Lemma 9.8. Moreover, by [14], Lemma 6.2, there exists a dense open subset $\Omega$ of $\mathfrak{a}_{P \mathrm{qC}}^{*}$ such that, for $\nu \in \Omega$, the element $\nu-\rho_{P}$ is a leading exponent of $f_{\nu}$ along $(P, v)$. Hence, by [14], Lemma 4.12, it follows that, for $\nu \in \Omega, X \in \mathfrak{a}_{P \mathrm{q}}$ and $m \in \mathrm{X}_{P, v,+}$,

$$
q_{\nu-\rho_{P}}(P, v \mid D f)(X, \nu, m)=\mu_{P, v}(D) \varphi_{\nu}(\exp X m)
$$


where the function $\varphi_{\nu}: \mathrm{X}_{1 P, v,+} \rightarrow V_{\tau}$ is defined by

$$
\varphi_{\nu}(m a)=a^{\nu} q_{\nu-\rho_{P}}\left(P, v \mid f_{\nu}, \log a, \nu, m\right),
$$

for $a \in A_{P \mathrm{q}}$ and $m \in \mathrm{X}_{P, v,+}$. By Proposition 13.6 the expression on the righthand side of (14.4) equals $a^{\nu} \psi_{v}(m)$, and we see that

$$
q_{\nu-\rho_{P}}(P, v \mid D f)(X, \nu, m)=\mu_{P, v}(D: \nu) \psi_{v}(m),
$$

for $\nu$ in a dense open subset of $\mathfrak{a}_{P \mathrm{qc}}^{*}, m \in \mathrm{X}_{P, v,+}$ and $X \in \mathfrak{a}_{P \mathrm{q}}$.

On the other hand, $\nu \mapsto \underline{\mu}_{P}(D: \nu) \psi$ is a polynomial $\mathcal{A}_{2, P}$-valued function on $\mathfrak{a}_{P \mathrm{qc}}^{*}$. It readily follows that the family

$$
g:(\nu, x) \mapsto E^{\circ}(P: \nu: x) \underline{\mu}_{P}(D: \nu) \psi
$$

belongs to $\mathcal{E}_{P}^{\text {hyp }}(\mathrm{X}: \tau)$. Moreover, by Proposition 13.6 it follows that

$$
q_{\nu-\rho_{P}}(P, v \mid g)(X, \nu, m)=\mu_{P, v}(D: \nu) \psi_{v}(m),
$$

for each $v \in{ }^{P} \mathcal{W}, \nu$ in a dense open subset of $\mathfrak{a}_{P \mathrm{qC}}^{*}$, all $X \in \mathfrak{a}_{P \mathrm{q}}$ and all $m \in$ $\mathrm{X}_{P, v,+}$. It follows from (14.5) and (14.6) that the family $D f-g \in \mathcal{E}_{P}^{\text {hyp }}(\mathrm{X}: \tau)$ satisfies all hypotheses of Theorem [6.11] Therefore, $D f=g$.

In the rest of this section we shall study the eigenvalues of the endomorphism $\underline{\mu}_{P}(D: \nu)$ of $\mathcal{A}_{2, P}$. For a start, we collect some facts about the action of $\mathbb{D}(\mathrm{X})$ on $\mathcal{A}_{2}(\mathrm{X}: \tau)$.

Let $L_{d}^{2}(\mathrm{X})$ be the discrete part of $L^{2}(\mathrm{X})$, defined as in the beginning of Section 12] It follows from [2, Thm. 1.5, that the space $L_{d}^{2}(\mathrm{X})$ admits a decomposition as an orthogonal direct sum of closed $G$-invariant subspaces on each of which $\mathbb{D}(\mathrm{X})$ acts by scalars (in the distribution sense). Let $\mathfrak{b}$ be a $\theta$-stable Cartan subspace of $\mathfrak{q}$. We denote by $\mathrm{L}(\mathrm{X}, \mathfrak{b})$ the collection of infinitesimal characters $\Lambda \in \mathfrak{b}_{\mathbb{C}}^{*}$ for which the associated character $\gamma(\cdot: \Lambda)=\gamma_{\mathfrak{b}}(\cdot: \Lambda)$ of $\mathbb{D}(\mathrm{X})$ occurs as a simultaneous eigenvalue in the decomposition mentioned.

The elements of the $\mathbb{D}(\mathrm{X})$-module $\mathcal{A}_{2}(\mathrm{X}: \tau)$ are $\mathbb{D}(\mathrm{X})$-finite and belong to $L_{d}^{2}(\mathrm{X}) \otimes V_{\tau}$. It follows that $\mathcal{A}_{2}(\mathrm{X}: \tau)$ splits as an algebraic direct sum of $\mathbb{D}(\mathrm{X})$ submodules on which the action of $\mathbb{D}(\mathrm{X})$ is by infinitesimal characters from $\mathrm{L}(\mathrm{X}, \mathfrak{b})$. More precisely, for $\Lambda \in \mathfrak{b}_{\mathbb{C}}^{*}$ we put

$$
\mathcal{A}_{2}(\mathrm{X}: \tau: \Lambda):=\left\{f \in \mathcal{A}_{2}(\mathrm{X}: \tau) \mid D f=\gamma(D: \Lambda) f, \quad \forall D \in \mathbb{D}(\mathrm{X})\right\} .
$$

This space is finite dimensional by [2, Lemma 3.9. It depends on $\Lambda$ through its class $[\Lambda]$ in $\mathfrak{b}_{\mathbb{C}}^{*} / W(\mathfrak{b})$; we therefore also denote it with $[\Lambda]$ in place of $\Lambda$. Let $\mathrm{L}(\mathfrak{b}, \tau)=\mathrm{L}(\mathrm{X}, \mathfrak{b}, \tau)$ denote the collection of $\Lambda \in \mathfrak{b}_{\mathbb{C}}^{*}$ for which $\mathcal{A}_{2}(\mathrm{X}: \tau: \Lambda) \neq 0$. Then $\mathrm{L}(\mathfrak{b}, \tau)$ is a $W(\mathfrak{b})$-invariant subset of $\mathrm{L}(\mathrm{X}, \mathfrak{b})$ and we have the following algebraic direct sum decomposition into joint eigenspaces for $\mathbb{D}(\mathrm{X})$,

$$
\mathcal{A}_{2}(\mathrm{X}: \tau)=\bigoplus_{\Lambda \in \mathrm{L}(\mathfrak{b}, \tau) / W(\mathfrak{b})} \mathcal{A}_{2}(\mathrm{X}: \tau: \Lambda)
$$


The summands in this decomposition are finite dimensional and mutually orthogonal with respect to the inner product from $L^{2}(\mathrm{X}: \tau)$. Moreover, the decomposition is finite by Lemma [12.6 In the chain of reasoning leading up to Theorem 21.2 finiteness of the decomposition requires $(G, H)$ to be of residue type, see Remark 12.2

Lemma 14.2 Let $\mathfrak{b}_{1}, \mathfrak{b}_{2} \subset \mathfrak{q}$ be two $\theta$-stable Cartan subspaces. Each element $s$ from $W\left(\mathfrak{b}_{2} \mid \mathfrak{b}_{1}\right)$, which set is non-empty by Lemma 3.8 maps $\mathrm{L}\left(\mathfrak{b}_{1}, \tau\right)$ bijectively onto $\mathrm{L}\left(\mathfrak{b}_{2}, \tau\right)$.

Proof: This follows by application of Lemma 3.8

Lemma 14.3 Let $P \in \mathcal{P}_{\sigma}$ and $v \in N_{K}\left(\mathfrak{a}_{\mathrm{q}}\right)$. Then $\mathfrak{a}_{P} \cap \operatorname{Ad}(v) \mathfrak{q}=\mathfrak{a}_{P \mathfrak{q}}$.

Proof: $\mathfrak{a}_{P} \cap \operatorname{Ad}(v) \mathfrak{q}=\operatorname{Ad}(v)\left(\mathfrak{a}_{v^{-1} P v} \cap \mathfrak{q}\right)=\operatorname{Ad}(v)\left(\mathfrak{a}_{v^{-1} P v} \cap \mathfrak{a}_{\mathrm{q}}\right)=\mathfrak{a}_{P} \cap \mathfrak{a}_{\mathrm{q}}=\mathfrak{a}_{P \mathrm{q}}$.

Let now $\mathfrak{b}$ be a Cartan subspace of $\mathfrak{q}$ containing $\mathfrak{a}_{\mathrm{q}}$ and let $v \in N_{K}\left(\mathfrak{a}_{\mathrm{q}}\right)$. Then $\mathfrak{b}^{v}:=\operatorname{Ad}(v) \mathfrak{b}$ is a Cartan subspace of $\operatorname{Ad}(v) \mathfrak{q}$, which contains $\mathfrak{a}_{\mathrm{q}}$. In particular, $\mathfrak{b}^{v}$ contains $\mathfrak{a}_{P \mathrm{q}}$, hence is contained in the latter's centralizer $\mathfrak{m}_{1 P}$. We write ${ }^{*} \mathfrak{b}_{P, v}:=\mathfrak{b}^{v} \cap \mathfrak{m}_{P}$. Then

$$
\mathfrak{b}^{v}={ }^{*} \mathfrak{b}_{P, v} \oplus \mathfrak{a}_{P \mathrm{q}} .
$$

In view of Lemma 14.3 this is the analogue of the decomposition (2.6) for the Cartan subspace $\mathfrak{b}^{v}$ related to symmetric pair $\left(\mathfrak{m}_{1 P}, \mathfrak{m}_{1 P} \cap \operatorname{Ad}(v) \mathfrak{h}\right)$. The restriction of $\operatorname{Ad}(v)$ to $\mathfrak{b}$ determines an element of $\operatorname{Hom}\left(\mathfrak{b}, \mathfrak{b}^{v}\right)$ that we denote by $\bar{v}$. The restriction $\left.\bar{v}\right|_{\mathfrak{a}_{\mathrm{q}}}$ is an element of $W$. The latter set equals $W\left(\mathfrak{a}_{\mathrm{q}} \mid \mathfrak{a}_{\mathrm{q}}\right)$, by Corollary 3.5. hence, by Lemma 3.7 applied with $\mathfrak{b}, \mathfrak{a}_{\mathrm{q}}, \mathfrak{a}_{\mathrm{q}}$ in place of $\mathfrak{d}, \mathfrak{b}_{1}, \mathfrak{b}_{2}$, there exists an element $s \in W(\mathfrak{b})$ such that $s=\bar{v}$ on $\mathfrak{a}_{\mathrm{q}}$. It readily follows that $\bar{v} \circ s^{-1} \in \operatorname{Hom}\left(\mathfrak{b}, \mathfrak{b}^{v}\right)$ equals the identity on $\mathfrak{a}_{P \mathfrak{q}}$, hence maps ${ }^{*} \mathfrak{b}_{P}$ isomorphically onto ${ }^{*} \mathfrak{b}_{P, v}$. Note that this map maps $W\left({ }^{*} \mathfrak{b}_{P}\right)$-orbits onto $W\left({ }^{*} \mathfrak{b}_{P, v}\right)$-orbits. The induced map from ${ }^{*} \mathfrak{b}_{P \mathbb{C}}^{*} / W\left({ }^{*} \mathfrak{b}_{P}\right)$ to $\left({ }^{*} \mathfrak{b}_{P, v}\right)_{\mathbb{C}}^{*} / W\left({ }^{*} \mathfrak{b}_{P, v}\right)$ is bijective and depends on $v$, but is independent of the particular choice of $s$. Given $\Lambda \in{ }^{*} \mathfrak{b}_{P \mathbb{C}}^{*}$, we define

$$
\mathcal{A}_{2}\left(\mathrm{X}_{P, v}: \tau_{P}: \Lambda\right):=\mathcal{A}_{2}\left(\mathrm{X}_{P, v}: \tau_{P}: \bar{v} \circ s^{-1} \Lambda\right) .
$$

Moreover, we define $\mathrm{L}_{P, v}(\mathfrak{b}, \tau)$ to be the set of $\Lambda \in{ }^{*} \mathfrak{b}_{P \mathbb{C}}^{*}$ for which the above space is non-trivial. Then

$$
\bar{v} \circ s^{-1} \mathrm{~L}_{P, v}(\mathfrak{b}, \tau)=\mathrm{L}\left(\mathrm{X}_{P, v},{ }^{*} \mathfrak{b}_{P, v}, \tau_{P}\right) .
$$

Thus, $\mathrm{L}_{P, v}(\mathfrak{b}, \tau)$ is a $W\left({ }^{*} \mathfrak{b}_{P}\right)$-invariant subset of ${ }^{*} \mathfrak{b}_{P \mathbb{C}}^{*}$.

Corollary 14.4 (RT) Let $P \in \mathcal{P}_{\sigma}$ and let $\mathfrak{b} \subset \mathfrak{q}$ be a $\theta$-stable Cartan subspace containing $\mathfrak{a}_{\mathrm{q}}$. Then

$$
\mathcal{A}_{2, P}=\bigoplus_{v \in P \mathcal{W}} \bigoplus_{\Lambda \in \mathrm{L}_{P, v}(\mathfrak{b}, \tau) / W\left(*^{*} \mathfrak{b}_{P}\right)} \mathrm{i}_{P, v} \mathcal{A}_{2}\left(\mathrm{X}_{P, v}: \tau_{P}: \Lambda\right),
$$


with a finite orthogonal direct sum of finite dimensional spaces. If $D \in \mathbb{D}(\mathrm{X})$ and $\nu \in \mathfrak{a}_{P \mathrm{qC}}^{*}$, then for every $v \in{ }^{P} \mathcal{W}$ and $\Lambda \in \mathrm{L}_{P, v}(\mathfrak{b}, \tau)$,

$$
\underline{\mu}_{P, v}(D: \nu)=\gamma_{\mathfrak{b}}(D: \Lambda+\nu) I \quad \text { on } \quad \mathrm{i}_{P, v} \mathcal{A}_{2}\left(\mathrm{X}_{P, v}: \tau_{P}: \Lambda\right) .
$$

Proof: We give the proof under the assumption that $P$ is of residue type, see Remark 12.2. By (13.1) the space $\mathcal{A}_{2, P}$ is the orthogonal direct sum of the spaces $\mathcal{A}_{2}\left(\mathrm{X}_{P, v}: \tau_{P}\right)$, as $v \in{ }^{P} \mathcal{W}$. Fix $v \in{ }^{P} \mathcal{W}$. By the assumption on $P$, the pair $\left(M_{P}, M_{P} \cap v P v^{-1}\right)$ is of residue type, hence $\mathcal{A}_{2}\left(\mathrm{X}_{P, v}: \tau_{P}\right)$ is finite dimensional and by (14.7) it is the orthogonal direct sum of the spaces $\mathcal{A}_{2}\left(\mathrm{X}_{P, v}: \tau_{P}: \Lambda^{\prime}\right)$, with $\Lambda^{\prime} \in \mathrm{L}\left(\mathrm{X}_{P, v},{ }^{*} \mathfrak{b}_{P, v}, \tau_{P}\right) / W\left({ }^{*} \mathfrak{b}_{P, v}\right)$. It now follows from (14.8) and (14.9) that $\mathcal{A}_{2}\left(\mathrm{X}_{P, v}: \tau_{P}\right)$ is the orthogonal direct sum of the spaces $\mathcal{A}_{2}\left(\mathrm{X}_{P, v}: \tau_{P}: \Lambda\right)$, for $\Lambda \in \mathrm{L}_{P, v}(\mathfrak{b}, \tau) / W\left({ }^{*} \mathfrak{b}_{P}\right)$; moreover, the sum is finite and the summands are finite dimensional. This establishes (14.10), with the asserted properties.

Let $\Lambda \in \mathrm{L}_{P, v}(\mathfrak{b}, \tau)$. Then by (14.9), $\Lambda^{\prime}:=\bar{v} s^{-1} \Lambda$ belongs to $\mathrm{L}\left(\mathrm{X}_{P, v},{ }^{*} \mathfrak{b}_{P, v}, \tau_{P}\right)$. Let now $\psi \in \mathcal{A}_{2}\left(\mathrm{X}_{P, v}: \tau_{P}: \Lambda\right)$. Then, writing $D^{v}=\operatorname{Ad}(v) D$ for $D \in \mathbb{D}(\mathrm{X})$,

$$
\begin{aligned}
\underline{\mu}_{P, v}(D: \nu) \psi & =\mu_{P}^{v}\left(D^{v}: \nu\right) \psi \\
& =\gamma_{* \mathfrak{b}_{P, v}}^{\mathrm{X}_{P}}\left(\mu_{P}^{v}\left(D^{v}: \nu\right): \Lambda^{\prime}\right) \psi \\
& =\gamma_{\mathfrak{b}^{v}}^{\mathrm{X}_{1 P, v}}\left(\mu_{P}^{v}\left(D^{v}\right): \Lambda^{\prime}+\nu\right) \psi .
\end{aligned}
$$

In the last equation we have used that $\gamma_{\mathfrak{b}^{v}}^{\mathrm{X}_{1 P, v}}=\gamma_{* \mathfrak{b}_{P, v}}^{\mathrm{X}_{P, v}} \otimes I$, in accordance with (14.3). Combining (14.1) for the triple $\left(G / v H v^{-1}, \mathfrak{b}^{v}, P\right)$ in place of $(G / H, \mathfrak{b}, P)$ with (14.2), we obtain that

$$
\begin{aligned}
\underline{\mu}_{P, v}(D: \nu) \psi & =\gamma_{\mathfrak{b} v}^{G / v H v^{-1}}\left(D^{v}: \Lambda^{\prime}+\nu\right) \psi \\
& =\gamma_{\mathfrak{b}}\left(D: \operatorname{Ad}(v)^{-1}\left(\Lambda^{\prime}+\nu\right)\right) \psi \\
& =\gamma_{\mathfrak{b}}\left(D: s \circ \operatorname{Ad}(v)^{-1}\left(\Lambda^{\prime}+\nu\right)\right) \psi \\
& =\gamma_{\mathfrak{b}}(D: \Lambda+\nu) \psi .
\end{aligned}
$$

We define $\mathrm{L}_{P}(\mathfrak{b}, \tau) \subset{ }^{*} \mathfrak{b}_{P \mathbb{C}}^{*}$ to be the union of the sets $\mathrm{L}_{P, v}(\mathfrak{b}, \tau)$, for $v \in{ }^{P} \mathcal{W}$. Moreover, for $\Lambda$ in this union, we put

$$
\mathcal{A}_{2, P}(\Lambda):=\bigoplus_{v \in{ }^{P} \mathcal{W}} \mathrm{i}_{P, v} \mathcal{A}_{2}\left(\mathrm{X}_{P, v}: \tau_{P}: \Lambda\right) .
$$

Corollary 14.5 (RT) Let $P \in \mathcal{P}_{\sigma}$. Then

$$
\mathcal{A}_{2, P}=\bigoplus_{\Lambda \in \mathrm{L}_{P}(\mathfrak{b}, \tau) / W\left({ }^{*} \mathfrak{b}_{P}\right)} \mathcal{A}_{2, P}(\Lambda) .
$$

Moreover, if $\Lambda \in \mathrm{L}_{P}(\mathfrak{b}, \tau)$ and $\psi \in \mathcal{A}_{2, P}(\Lambda)$, then, for every $D \in \mathbb{D}(\mathrm{X})$,

$$
D E^{\circ}(P: \nu) \psi=\gamma_{\mathfrak{b}}(D: \Lambda+\nu) E^{\circ}(P: \nu) \psi,
$$

as a meromorphic $C^{\infty}(\mathrm{X}: \tau)$-valued identity in $\nu \in \mathfrak{a}_{P \mathrm{qC}}^{*}$. 
Proof: We give the proof under the assumption that $P$ is of residue type, see Remark 12.2 The result follows from Corollary 14.4 combined with Lemma 14.1

We end this section with a description of the action of $\mathbb{D}(\mathrm{X})$ on the dualized Eisenstein integral. For $D \in \mathbb{D}(\mathrm{X})$ we define the formal adjoint $D^{*} \in \mathbb{D}(\mathrm{X})$ by

$$
\langle D f \mid g\rangle=\left\langle f \mid D^{*} g\right\rangle,
$$

for all $f, g \in C_{c}^{\infty}(\mathrm{X})$; here $\langle\cdot \mid \cdot\rangle$ denotes the inner product from $L^{2}(\mathrm{X})$. The canonical anti-automorphism $X \mapsto X^{\vee}$ of $U(\mathfrak{g})$ induces an anti-automorphism of $U(\mathfrak{g})^{H} / U(\mathfrak{g})^{H} \cap U(\mathfrak{g}) \mathfrak{h} \simeq \mathbb{D}(\mathrm{X})$, which we also denote by $D \mapsto D^{\vee}$. If $D \in \mathbb{D}(\mathrm{X})$, let $\bar{D}$ be its complex conjugate, i.e. the differential operator with complex conjugate coefficients. Then $D^{*}=\bar{D}^{\vee}$, for every $D \in \mathbb{D}(\mathrm{X})$.

We recall from [3], Lemma 7.2, that $D$ restricts to a continuous linear endomorphism of $\mathcal{C}(X)$; by density of $C_{c}^{\infty}(\mathrm{X})$ in $\mathcal{C}(\mathrm{X})$ it follows that (14.11) also holds for all $f, g \in \mathcal{C}(\mathrm{X})$.

Lemma 14.6 Let $P \in \mathcal{P}_{\sigma}, v \in N_{K}\left(\mathfrak{a}_{\mathrm{q}}\right)$ and $D \in \mathbb{D}(\mathrm{X})$. Then

$$
\mu_{P, v}\left(D^{*}\right)=\mu_{P, v}(D)^{*} .
$$

Proof: We note that $\mu_{P, e}=\mu_{P}$; hence, for $v=e$, the result follows by the same argument as in [5], proof of Lemma 19.3. For general $v$ the result follows by application of (14.2).

Lemma 14.7 (RT) Let $P \in \mathcal{P}_{\sigma}$. Then, for every $D \in \mathbb{D}(\mathrm{X})$ and all $\nu \in \mathfrak{a}_{P \mathrm{qC}}^{*}$,

$$
\underline{\mu}_{P}(D: \nu)^{*}=\underline{\mu}_{P}\left(D^{*}:-\bar{\nu}\right) .
$$

Proof: We give the proof under the assumption that $P$ is of residue type, see Remark 12.2 Let $v \in{ }^{P} \mathcal{W}$. The decomposition $\mathrm{X}_{1 P, v} \simeq \mathrm{X}_{P, v} \times A_{P \mathrm{q}}$ induces an isomorphism $\mathbb{D}\left(\mathrm{X}_{1 P, v}\right) \simeq \mathbb{D}\left(\mathrm{X}_{P, v}\right) \otimes S\left(\mathfrak{a}_{P \mathrm{q}}\right)$. Accordingly $(u \otimes p)^{*}=u^{*} \otimes p^{*}$, for all $u \in \mathbb{D}\left(\mathrm{X}_{P, v}\right)$ and $p \in S\left(\mathfrak{a}_{P \mathrm{q}}\right)$. Moreover, $p^{*}(\nu)=\overline{p(-\bar{\nu})}$, for $\nu \in \mathfrak{a}_{P \mathrm{qc}}^{*}$. Hence, $(u \otimes p)^{*}(\nu)=\bar{p}(-\bar{\nu}) u^{*}=[u \otimes p(-\bar{\nu})]^{*}$ and we see that $u^{*}(\nu)=u(-\bar{\nu})^{*}$ for $u \in \mathbb{D}\left(\mathrm{X}_{1 P, v}\right)$ and $\nu \in \mathfrak{a}_{P \mathrm{qC}}^{*}$. Applying this to (14.12) it follows that for $D \in \mathbb{D}(\mathrm{X})$ and $\nu \in \mathfrak{a}_{P \mathrm{qC}}^{*}$ we have $\mu_{P, v}(D: \nu)^{*}=\mu_{P, v}\left(D^{*}:-\bar{\nu}\right)$. By the argument in the text preceding Lemma 14.6 applied to $\mathrm{X}_{P, v}$ in place of $\mathrm{X}$, we infer that

$$
\left\langle\mu_{P, v}(D: \nu) f \mid g\right\rangle=\left\langle f \mid \mu_{P, v}\left(D^{*}:-\bar{\nu}\right) g\right\rangle,
$$

for all $f, g \in \mathcal{C}\left(\mathrm{X}_{P, v}: \tau_{P}\right)$. Here $\langle\cdot \mid \cdot\rangle$ denotes the $L^{2}$-inner product. In particular, the equation holds for $f, g$ in the subspace $\mathcal{A}_{2}\left(\mathrm{X}: \tau_{P}\right)$, which is finite dimensional, since $P$ is of residue type. Hence, $\underline{\mu}_{P, v}(D: \nu)^{*}=\underline{\mu}_{P, v}\left(D^{*}:-\bar{\nu}\right)$. By orthogonality of the direct sum decomposition in (13.1), the result follows by summation over $v \in{ }^{P} \mathcal{W}$. 
Lemma 14.8 (RT) Let $P \in \mathcal{P}_{\sigma}$. Then for every $D \in \mathbb{D}(\mathrm{X})$,

$$
D E^{*}(P: \nu)=\underline{\mu}_{P}\left(D^{\vee}: \nu\right) E^{*}(P: \nu),
$$

as a meromorphic identity in $\nu \in \mathfrak{a}_{P \mathrm{qc}}^{*}$.

Proof: We give the proof under the assumption that $P$ is of residue type, see Remark 12.2 By linearity, we may assume that $D$ is real. It then follows from the definition of the dual Eisenstein integral, see Definition 13.7 combined with Lemma 14.1 that

$$
D E^{*}(P: \nu)=\underline{\mu}_{P}(D:-\bar{\nu})^{*} E^{*}(P: \nu) .
$$

The lemma now follows by application of Lemma 14.7 in view of the fact that $D^{*}=D^{\vee}$.

\section{Uniform tempered estimates}

In this section we present straightforward generalizations of results of [5], Sect. 18 , to a setting involving families $\left\{f_{\nu}\right\}$ of eigenfunctions on $\mathrm{X}$, with holomorphic dependence on a parameter $\nu \in \mathfrak{a}_{Q \mathrm{qc}}^{*}$, where $Q \in \mathcal{P}_{\sigma}$. A similar generalization has been given in 22, Sect. 9. The generalized results allow us to sharpen uniformly moderate estimates of type (13.9) to uniform tempered estimates. In particular, we obtain estimates of the latter type for the normalized Eisenstein integral.

We fix $Q \in \mathcal{P}_{\sigma}$, a $\theta$-stable Cartan subspace $\mathfrak{b}$ of $\mathfrak{q}$ containing $\mathfrak{a}_{\mathrm{q}}$ and an element $\Lambda \in{ }^{*} \mathfrak{b}_{Q \mathbb{C}}^{*}$, cf. (2.6). For $\varepsilon>0$, we put

$$
\mathfrak{a}_{Q \mathrm{q}}^{*}(\varepsilon):=\left\{X \in \mathfrak{a}_{Q \mathrm{qC}}^{*}|\quad| \operatorname{Re} X \mid<\varepsilon\right\} .
$$

The closure of this set is denoted by $\overline{\mathfrak{a}}_{Q \mathrm{q}}^{*}(\varepsilon)$.

Definition 15.1 Let $\varepsilon>0$. We define $\mathcal{E}(Q: \Lambda: \varepsilon)=\mathcal{E}(\mathrm{X}: Q: \Lambda: \varepsilon)$ to be the space of smooth functions $f: \mathfrak{a}_{Q \mathrm{q}}^{*}(\varepsilon) \times \mathrm{X} \rightarrow \mathbb{C}$ satisfying the following conditions.

(a) The function $f$ is holomorphic in its first variable.

(b) For every $\nu \in \mathfrak{a}_{Q \mathrm{q}}^{*}(\varepsilon)$, the function $f_{\nu}: x \mapsto f(\nu, x)$ satisfies the following system of differential equations

$$
D f_{\nu}=\gamma(D: \Lambda+\nu) f_{\nu}, \quad(D \in \mathbb{D}(\mathrm{X})) .
$$

Note that in this definition it is not required that $f$ is $K$-finite, or spherical, from the left. We also have the following analogue of the space $\mathcal{M}(\Lambda, \varepsilon)$ defined in [5], Sect. 18. 
Definition 15.2 Let $\varepsilon>0$. A function $f \in \mathcal{E}(Q: \Lambda: \varepsilon)$ is called uniformly moderate of exponential rate $r \geq 0$ if for every $u \in U(\mathfrak{g})$ there exist constants $n \in \mathbb{N}$ and $C>0$ such that

$$
\left\|L_{u} f_{\nu}(x)\right\| \leq C(1+|\nu|)^{n} e^{r l \times}(x),
$$

for all $\nu \in \mathfrak{a}_{Q \mathrm{q}}^{*}(\varepsilon)$ and $x \in \mathrm{X}$. The space of all such functions is denoted by $\mathcal{E}^{\mathrm{um}}(Q: \Lambda: \varepsilon: r)$.

Lemma 15.3 (RT) Let $Q \in \mathcal{P}_{\sigma}$ and let $\varepsilon>0$. There exists a polynomial function $p \in \Pi_{\Sigma_{r}(Q), \mathbb{R}}\left(\mathfrak{a}_{Q \mathrm{q}}^{*}\right)$ such that the $C^{\infty}(\mathrm{X}) \otimes \operatorname{Hom}\left(\mathcal{A}_{2, Q}, V_{\tau}\right)$-valued meromorphic function $\nu \mapsto p(\nu) E^{\circ}(Q: \nu)$ is regular on $\overline{\mathfrak{a}}_{Q \mathrm{q}}^{*}(\varepsilon)$ and such that the following holds. There exists a constant $r>0$ such that for every $\Lambda \in \mathrm{L}_{Q}(\mathfrak{b}, \tau)$, $\psi \in \mathcal{A}_{2, Q}(\Lambda)$ and $\eta \in V_{\tau}^{*}$, the family $f:(\nu, x) \mapsto \eta\left(p(\nu) E^{\circ}(Q: \nu: x)\right)$ belongs to $\mathcal{E}^{\mathrm{um}}(Q: \Lambda: \varepsilon: r)$.

Proof: We give the proof under the assumption that $Q$ is of residue type, see Remark 12.2] Let $R>0$ be such that $\mathfrak{a}_{Q \mathrm{q}}^{*}(\varepsilon) \subset \mathfrak{a}_{Q \mathrm{q}}^{*}(\bar{Q}, R)$. Then by Proposition 13.14 there exists a polynomial function $p \in \Pi_{\Sigma_{r}(Q)}\left(\mathfrak{a}_{Q \mathrm{q}}^{*}\right)$ such that the $C^{\infty}(\mathrm{X}) \otimes \operatorname{Hom}\left(\mathcal{A}_{2, Q}, V_{\tau}\right)$-valued meromorphic function $F: \nu \mapsto p(\nu) E^{\circ}(Q: \nu)$ is holomorphic on a neighborhood of $\overline{\mathfrak{a}}_{Q \mathrm{q}}^{*}(\bar{Q}, R)$. Moreover, there exists $r^{\prime}>0$ and for every $u \in U(\mathfrak{g})$ constants $n \in \mathbb{N}$ and $C>0$ such that

$$
\left\|L_{u} F_{\nu}(x)\right\| \leq C(1+|\nu|)^{n} e^{\left(r^{\prime}+|\operatorname{Re} \nu|\right) l_{\mathrm{X}}(x)},
$$

for $x \in \mathrm{X}, \nu \in \overline{\mathfrak{a}}_{Q \mathrm{q}}^{*}(\bar{Q}, R)$. Put $r=r^{\prime}+\varepsilon$. Then it follows that $F$ is holomorphic on a neighborhood of $\overline{\mathfrak{a}}_{Q \mathrm{q}}^{*}(\varepsilon)$ and satisfies the estimates

$$
\left\|L_{u} F_{\nu}(x)\right\| \leq C(1+|\nu|)^{n} e^{r l \times}(x)
$$

for $x \in \mathrm{X}$ and $\nu \in \mathfrak{a}_{Q \mathrm{q}}^{*}(\varepsilon)$. Let $f$ be defined as in the lemma. Then $L_{u} f_{\nu}(x)=$ $\eta\left(L_{u} F_{\nu}(x) \psi\right)$. Hence, it follows from the above and from Corollary 14.5 that $f \in$ $\mathcal{E}(Q: \Lambda: \varepsilon)$. Finally, it follows from the estimates (15.1) that $f \in \mathcal{E}^{\mathrm{um}}(Q: \Lambda: \varepsilon: r)$.

We also have the following obvious generalization of the notion of uniformly tempered families; see [5], Sect. 18. For $\nu \in \mathfrak{a}_{Q \mathrm{qC}}^{*}$ and $x \in \mathrm{X}$ we put

$$
|(\nu, x)|:=(1+|\nu|)\left(1+l_{\mathrm{X}}(x)\right) .
$$

Definition 15.4 Let $\varepsilon>0$. A function $f \in \mathcal{E}(Q: \Lambda: \varepsilon)$ is called uniformly tempered of scale $s$ if for every $u \in U(\mathfrak{g})$ there exist constants $n \in \mathbb{N}$ and $C>0$ such that

$$
\left|L_{u} f_{\nu}(x)\right| \leq C|(\nu, x)|^{n} \Theta(x) e^{s|\operatorname{Re} \nu| l_{\mathrm{X}}(x)},
$$

for all $\nu \in \mathfrak{a}_{Q \mathrm{q}}^{*}(\varepsilon)$ and all $x \in \mathrm{X}$. The space of all such functions is denoted by $\mathcal{T}(Q: \Lambda: \varepsilon: s)$. 
If $f \in \mathcal{E}^{\text {um }}(Q: \Lambda: \varepsilon: r)$, then for every $\nu \in \mathfrak{a}_{Q \mathrm{qC}}^{*}$ the function $f_{\nu}$ belongs to the space $\mathcal{E}_{\Lambda+\nu, *}^{\infty}(\mathrm{X})$, defined in [5], p. 392, see also p. 387. If $g$ is any function in the latter space, then, viewed as a function on $G$, it has an asymptotic expansion along every parabolic subgroup $P \in \mathcal{P}_{\sigma}$ of the form

$$
g(x \exp t X) \sim \sum_{\xi \in Z-\mathbb{N} \Delta_{r}(P)} p_{\xi}(P \mid g, x, X) e^{t \xi(X)},
$$

as $t \rightarrow \infty$, for $x \in G$ and $X \in \mathfrak{a}_{P \mathrm{q}}^{+}$. Here $Z$ is a finite subset of $\mathfrak{a}_{P \mathrm{qC}}^{*}$ and there exists a $d \in \mathbb{N}$ such that the $p_{\xi}(P \mid g)$ are smooth functions $G \rightarrow P_{d}\left(\mathfrak{a}_{P q}\right)$, for all $\xi$. Moreover, the functions $p_{\xi}(P \mid g)$ are uniquely determined, see [5], Theorem 12.8. Accordingly, we may define the set of exponents of $g$ along the parabolic subgroup $P$ by

$$
\operatorname{Exp}(P \mid g):=\left\{\xi \in Z-\mathbb{N} \Delta_{r}(P) \mid \quad p_{\xi}(P \mid g) \neq 0\right\} .
$$

We define the partial ordering $\preceq_{P}$ on $\mathfrak{a}_{P \text { qC }}^{*}$ by

$$
\lambda \preceq_{P} \mu \Longleftrightarrow \mu-\lambda \in \Delta_{r}(P), \quad\left(\lambda, \mu \in \mathfrak{a}_{P \mathrm{qC}}^{*}\right) .
$$

The $\preceq_{P}$-maximal elements in the set (15.2) are called the leading exponents of $g$ along $P$. The set of these leading exponents is denoted by $\operatorname{Exp}_{\mathrm{L}}(P \mid g)$.

Remark 15.5 The above notions of asymptotic coefficients and exponents are related to the similar notions introduced in $\S[$ as follows.

Let $f \in \mathcal{A}(\mathrm{X}: \tau)$ and assume that every vector component $\eta \circ f$, for $\eta \in V_{\tau}^{*}$, belongs to $\mathcal{E}_{\Lambda+\nu, *}^{\infty}(\mathrm{X})$. For $P \in \mathcal{P}_{\sigma}$, let $\operatorname{Exp}(f \mid P)$ denote the union of the sets $\operatorname{Exp}(\eta \circ f \mid P)$, for $\eta \in V_{\tau}^{*}$; by sphericality of $f$ this union equals the union with index $\eta$ ranging over any generating subset of the $K$-module $V_{\tau}^{*}$. If $u \in N_{K}\left(\mathfrak{a}_{\mathrm{q}}\right)$, then it readily follows from the definitions that $\operatorname{Exp}(P, u \mid f) \subset \operatorname{Exp}(P \mid f)$. Moreover, by uniqueness of asymptotics we have, for $\xi \in \operatorname{Exp}(P, u \mid f)$, that

$$
\eta\left(q_{\xi}(P, u \mid f, X, m)\right)=p_{\xi}(P \mid \eta \circ f, m u, X), \quad\left(m \in M_{P}, X \in \mathfrak{a}_{P q}\right),
$$

for all $\eta \in V_{\tau}^{*}$.

Lemma 15.6 Let $\nu \in \mathfrak{a}_{O \mathrm{qc}}^{*}$ and assume that $g \in \mathcal{E}_{\Lambda+\nu, *}^{\infty}(\mathrm{X})$. Let $P \in \mathcal{P}_{\sigma}^{\min }$. Then for every $\xi \in \operatorname{Exp}_{L}(P \mid g)$ there exists a $s \in W(\mathfrak{b})$ such that $\xi+\rho_{P}=$ $\left.s(\nu+\Lambda)\right|_{\mathfrak{a}_{\mathrm{q}}}$.

Proof: We recall that $\mathfrak{a}_{\mathrm{q}} \subset \mathfrak{b}$. Let $\Sigma^{+}(\mathfrak{b})$ be a choice of positive roots for $\Sigma(\mathfrak{b})$ that is compatible with $\Sigma(P)$. Let $\mathfrak{g}_{\mathbb{C}}^{+}$be the associated sum of the positive root spaces and let $\mathfrak{m}_{\mathbb{C}}^{+}$be its intersection with $\mathfrak{m}_{\mathbb{C}}$. Let $\delta:=\frac{1}{2} \operatorname{tr}\left[\left.\operatorname{ad}(\cdot)\right|_{\mathfrak{g}_{\mathbb{C}}^{+}}\right] \in \mathfrak{b}_{\mathbb{C}}^{*}$ and let $\delta_{0}:=\frac{1}{2} \operatorname{tr}\left[\left.\operatorname{ad}(\cdot)\right|_{\mathfrak{m}_{\mathrm{c}}^{+}}\right] \in i \mathfrak{b}_{\mathrm{k}}^{*}$. Then $\delta=\delta_{0}+\rho_{P}$.

Let $\xi$ be a leading exponent along $P$. Then by [5], Cor. 13.3 and Lemma 13.1, the function $\varphi \in C^{\infty}\left(M_{1}\right)$ defined by

$$
\varphi(m a)=a^{\xi} p_{\xi}(P \mid g, m, \log a), \quad\left(m \in M_{\sigma}, a \in A_{\mathrm{q}}\right),
$$


is right $M_{\sigma} \cap H$-invariant and satisfies the following system of differential equations

$$
\mu_{P}^{\prime}(D) \varphi=\gamma(D: \Lambda+\nu) \varphi, \quad(D \in \mathbb{D}(\mathrm{X})) .
$$

Here $\mu_{P}^{\prime}$ is defined as in [5], Sect. 2. Now $M_{\sigma} / M_{\sigma} \cap H \simeq M_{10} / M_{10} \cap H$, naturally, so that $\varphi$ may be viewed as a function in $C^{\infty}\left(\mathrm{X}_{10}\right)$. By (14.3) with $P=P_{0}$ and $v=e$ we have $\mathbb{D}\left(\mathrm{X}_{10}\right) \simeq \mathbb{D}\left(\mathrm{X}_{0}\right) \otimes S\left(\mathfrak{a}_{\mathrm{q}}\right)$. Since $p_{\xi}$ is polynomial in $\log a$, the second component of the tensor product acts on $\varphi$ with a single generalized eigenvalue $u \mapsto u(\xi)$. On the other hand, we recall from [5], Lemma 4.8, that the action of $\mathbb{D}\left(\mathrm{X}_{0}\right)$ on $C^{\infty}\left(\mathrm{X}_{0}\right)_{K_{0}}$ allows a simultaneous diagonalization with eigenvalues of the form $D \mapsto \gamma^{\mathrm{X}_{0}}\left(D: \Lambda_{0}+\delta_{0}\right)$, with $\Lambda_{0} \in i \mathfrak{b}_{\mathrm{k}}^{*}$. It follows that there exists a $\Lambda_{0} \in i \mathfrak{b}_{\mathrm{k}}^{*}$ such that

$$
\gamma^{\mathrm{X}_{0}}\left(\mu_{P}^{\prime}(D: \xi): \Lambda_{0}+\delta_{0}\right)=\gamma(D: \Lambda+\nu), \quad(D \in \mathbb{D}(\mathrm{X})) .
$$

The expression on the left-hand side of this expression can be rewritten as $\gamma_{P}\left(D: \Lambda_{0}+\xi+\delta_{0}+\rho_{P}\right)=\gamma\left(D: \Lambda_{0}+\xi+\delta\right)$, from which we conclude that $\Lambda_{0}+\xi+\delta \in W(\mathfrak{b})(\Lambda+\nu)$. Since $\left.\left(\Lambda_{0}+\delta\right)\right|_{\mathfrak{a}_{\mathrm{q}}}=\rho_{P}$, it follows that $\xi+\rho_{P}=$ $\left.s(\nu+\Lambda)\right|_{\mathfrak{a}_{\mathrm{q}}}$, for some $s \in W(\mathfrak{b})$.

We can now generalize [5], Theorem 18.3. For an appropriate formulation we need the following definition.

Definition 15.7 We say that the exponents of a family $f \in \mathcal{E}^{\mathrm{um}}(Q: \Lambda: \varepsilon: r)$ are tempered along a minimal $\sigma$-parabolic subgroup $P \in \mathcal{P}_{\sigma}^{\min }$ if for every $\nu \in \mathfrak{a}_{Q \mathrm{q}}^{*}(\varepsilon)$ the set of exponents $\operatorname{Exp}\left(P \mid f_{\nu}\right)$ satisfies the following condition. For every $\xi \in \operatorname{Exp}\left(P \mid f_{\nu}\right)$, there exists a $s \in W(\mathfrak{b})$ such that

(a) $\operatorname{Re}(s \Lambda) \leq 0$ on $\mathfrak{a}_{\mathrm{q}}^{+}(P)$,

(b) $\left.\xi \in s(\nu+\Lambda)\right|_{\mathfrak{a}_{\mathrm{q}}}-\rho_{P}-\mathbb{N} \Delta(P)$.

We denote by $\mathcal{E}_{\mathrm{T}}^{\mathrm{um}}(Q: \Lambda: \varepsilon: r)$ the space of functions $f \in \mathcal{E}^{\mathrm{um}}(Q: \Lambda: \varepsilon: r)$, such that for every $P \in \mathcal{P}_{\sigma}^{\min }$ the exponents of $f$ along $P$ are tempered.

Remark 15.8 If $Q$ is a minimal $\sigma$-parabolic subgroup, then it follows by application of [5], Thm. 13.7, that $\mathcal{E}^{\mathrm{um}}(Q: \Lambda: \varepsilon: r)=\mathcal{E}_{\mathrm{T}}^{\mathrm{um}}(Q: \Lambda: \varepsilon: r)$.

Theorem 15.9 Let $Q \in \mathcal{P}_{\sigma}$ and let $r>0$. Then there exists a $s>0$ such that for sufficiently small $\varepsilon>0$,

$$
\mathcal{E}_{\mathrm{T}}^{\mathrm{um}}(Q: \Lambda: \varepsilon: r) \subset \mathcal{T}(Q: \Lambda: \varepsilon: s) .
$$

Proof: The proof is a straightforward, but somewhat tedious, adaptation of the proof of [5], Theorem 18.3, with trivial alterations because of the change of the parameter set. Conditions (a) and (b) of Definition 15.7 are to be used in place of [5], Theorem 13.7, see the proof of [5], Proposition 18.14, to keep track of the exponents occurring in the asymptotic expansions considered. If $Q$ is minimal, then the mentioned Theorem 13.7 implies conditions (a) and (b) for any family $f \in \mathcal{E}^{\mathrm{um}}(Q: \Lambda: \varepsilon: r)$; see Remark 15.8 
Remark 15.10 Another version of Theorem [15.9] is given by [22, Thm. 3 . However, in that paper the requirement on the exponents in Definition 15.7 is replaced by the requirement that the function $f_{\nu}$ is tempered for every $\nu \in i \mathfrak{a}_{\mathrm{q}}^{*}$. By an additional argument it is then shown that this requirement is equivalent to the one of Definition 15.7 see 22], Lemma 23. We shall not need this result, since by Proposition 13.15 the needed information on the exponents is known for the normalized Eisenstein integrals to which Theorem 15.9 will be applied.

Definition 15.11 Let $Q \in \mathcal{P}_{\sigma}, \Lambda \in{ }^{*} \mathfrak{b}_{Q \mathbb{C}}^{*}, \varepsilon>0$ and $s>0$. Then by $\mathcal{T}(Q, \tau, \Lambda, \varepsilon, s)$ we denote the space of smooth functions $f: \mathfrak{a}_{Q \mathrm{q}}^{*}(\varepsilon) \times \mathrm{X} \rightarrow V_{\tau}$ such that

(a) for every $\eta \in V_{\tau}^{*}$ the family $\eta \circ f:(\nu, x) \mapsto \eta(f(\nu, x))$ belongs to $\mathcal{T}(Q, \Lambda, \varepsilon, s)$;

(b) $f_{\nu}$ is $\tau$-spherical for every $\nu \in \mathfrak{a}_{Q \mathrm{q}}^{*}(\varepsilon)$.

Theorem 15.12 (RT) Let $Q \in \mathcal{P}_{\sigma}$. There exists a polynomial function $p \in$ $\Pi_{\Sigma_{r}(Q), \mathbb{R}}\left(\mathfrak{a}_{Q \mathrm{q}}^{*}\right)$ and constants $s>0$ and $\varepsilon>0$ such that the meromorphic $C^{\infty}(\mathrm{X}) \otimes \operatorname{Hom}\left(\mathcal{A}_{2, Q}, V_{\tau}\right)$-valued function $\nu \mapsto p(\nu) E^{\circ}(Q: \nu)$ is holomorphic on $\mathfrak{a}_{Q \mathfrak{q}}^{*}(\varepsilon)$, and such that the following holds. For each $\Lambda \in \mathrm{L}_{Q}(\mathfrak{b}, \tau)$ and every $\psi \in \mathcal{A}_{2, Q}(\Lambda)$ the family $f:(\nu, x) \mapsto p(\nu) E^{\circ}(Q: \nu: x) \psi$ belongs to $\mathcal{T}(Q, \tau, \Lambda, \varepsilon, s)$.

Remark 15.13 For $Q$ minimal, this result is due [5], Thm. 19.2, in view of 9], Eqn. (52). For general $Q$, a similar result for an unnormalized version of the Eisenstein integral is due to [22, Thm. 4.

Proof: We give the proof under the assumption that $Q$ is of residue type, see Remark 12.2 Fix $\varepsilon>0$. There exist $p \in \Pi_{\Sigma_{r}(Q), \mathbb{R}}\left(\mathfrak{a}_{Q \mathrm{q}}^{*}\right)$ and $r>0$ as in Lemma 15.3 Fix $\Lambda \in \mathrm{L}_{Q}(\mathfrak{b}, \tau)$ and $\psi \in \mathcal{A}_{2, Q}(\Lambda)$. Define $f:(\nu, x) \mapsto p(\nu) E^{\circ}(Q: \nu: x) \psi$. Let $\eta \in V_{\tau}^{*}$ and define $F:(\nu, x) \mapsto \eta(f(\nu, x))$. Then by finite dimensionality of $\mathcal{A}_{2, Q}$ and $V_{\tau}$ it suffices to show that there exist $\varepsilon^{\prime}>0$ and $s>0$ such that $F \in \mathcal{T}\left(Q: \Lambda: \varepsilon^{\prime}: s\right)$.

In view of Theorem 15.9 it suffices to show that $F \in \mathcal{E}_{\mathrm{T}}^{\mathrm{um}}(Q: \Lambda: \varepsilon: r)$. In view of Lemma 15.3 the function $F$ belongs to $\mathcal{E}^{\mathrm{um}}(Q: \Lambda: \varepsilon: r)$. Let $P \in \mathcal{P}_{\sigma}^{\min }$. Then it remains to be verified that the exponents of $F$ along $P$ are tempered in the sense of Definition 15.7

There exists a $v \in N_{K}\left(\mathfrak{a}_{\mathrm{q}}\right)$ such that $P_{1}:=v^{-1} P v \subset Q$. The meromorphic $C^{\infty}(\mathrm{X}: \tau)$-valued function $\nu \mapsto f_{\nu}$ is regular on $\mathfrak{a}_{Q \mathrm{q}}^{*}(\varepsilon)$. Moreover, from Proposition 13.15 and [14, Lemma 3.6, it follows that, for $\nu \in \operatorname{reg} f$,

$$
\operatorname{Exp}\left(P, e \mid f_{\nu}\right)=v \operatorname{Exp}\left(P_{1}, v^{-1} \mid f_{\nu}\right) \subset v W^{P_{1} \mid Q}\left(\nu+\Lambda\left(P_{1} \mid Q\right)\right)-\rho_{P}-\mathbb{N} \Delta(P) .
$$

Thus, let $\nu_{0} \in \mathfrak{a}_{Q \mathrm{q}}^{*}(\varepsilon)$ be fixed, and let $\xi \in \operatorname{Exp}\left(P \mid F_{\nu_{0}}\right)$. Then we may select $s \in v W^{P_{1} \mid Q}$ and $\xi_{0} \in-s \Lambda\left(P_{1} \mid Q\right)+\mathbb{N} \Delta(P)$ such that $\xi=s \nu_{0}-\rho_{P}-\xi_{0}$. Since $f \in \mathcal{E}_{Q}(\mathrm{X}: \tau)$, see Definition 13.7 it follows from Definitions 6.6 6.4 and 6.3 that $f \in C_{Q, Y}^{\mathrm{ep}, \text { hyp }}\left(\mathrm{X}_{+}: \tau\right)$, for a suitable finite subset $Y \subset{ }^{*} \mathfrak{a}_{Q \mathrm{qc}}^{*}$. By Definition 6.1 and 14, Lemma 6.2, it follows that $s \nu-\rho_{P}-\xi_{0} \in \operatorname{Exp}\left(P, e \mid f_{\nu}\right)$, for $\nu$ in an open dense subset of $\mathfrak{a}_{Q \mathrm{qc}}^{*}$. 
Let $\xi_{1}$ be a $\preceq_{P}$-minimal element in $-s \Lambda\left(P_{1} \mid Q\right)+\mathbb{N} \Delta(P)$ with the property that $\xi_{1} \preceq_{P} \xi_{0}$ and that $s \nu-\xi_{1}-\rho_{P} \in \operatorname{Exp}\left(P, e \mid f_{\nu}\right)$ for $\nu$ in an open dense subset of $\mathfrak{a}_{Q \mathrm{qC}}^{*}$. Then for $\nu$ in an open dense subset $\Omega$ of $\mathfrak{a}_{Q \mathrm{qc}}^{*}$, the element $s \nu-\xi_{1}-\rho_{P}$ is a leading exponent of $f_{\nu}$ along $(P, e)$. By Lemma 15.6 it follows that

$$
s \nu-\xi_{1}-\left.\rho_{P} \in W(\mathfrak{b})(\nu+\Lambda)\right|_{\mathfrak{a}_{\mathrm{q}}}-\rho_{P},
$$

for $\nu \in \Omega$. This implies in turn that there exists $t \in W(\mathfrak{b})$ such that $s \nu-\xi_{1}=$ $\left.t(\nu+\Lambda)\right|_{\mathfrak{a}_{\mathrm{q}}}$, for all $\nu \in \mathfrak{a}_{Q \mathrm{qc}}^{*}$. Hence, $s \nu=\left.t \nu\right|_{\mathfrak{a}_{\mathrm{q}}}$ for all $\nu \in \mathfrak{a}_{Q \mathrm{qc}}^{*}$ and $-\xi_{1}=\left.t \Lambda\right|_{\mathfrak{a}_{\mathrm{q}}}$. Now $-\xi_{1} \in s \Lambda\left(P_{1} \mid Q\right)-\mathbb{N} \Delta(P) \subset-s\left(\mathbb{R}_{+} \Delta_{Q}\left(P_{1}\right)\right)-\mathbb{N} \Delta(P) \subset-\mathbb{R}_{+} \Delta(P)$, hence $\left.\operatorname{Re}(t \Lambda)\right|_{\mathfrak{a}_{\mathrm{q}}}=-\xi_{1} \leq 0$ on $\mathfrak{a}_{\mathrm{q}}^{+}(P)$. We complete the proof by observing that

$$
\xi=s \nu-\rho_{P}-\xi_{0}=s \nu-\xi_{1}-\rho_{P}-\left.\left(\xi_{0}-\xi_{1}\right) \in t(\nu+\Lambda)\right|_{\mathfrak{a}_{\mathrm{q}}}-\rho_{P}-\mathbb{N} \Delta(P) .
$$

\section{Infinitesimal characters of the discrete series}

In this section we describe a restriction on the set $\mathrm{L}(\mathrm{X}, \mathfrak{b})$ of $\mathbb{D}(\mathrm{X})$-characters of the discrete series, see the text before 14.7). The main result is due to $\mathrm{T}$. Oshima and T. Matsuki, 40.

Let $\mathfrak{b} \subset \mathfrak{q}$ be a $\theta$-stable Cartan subalgebra. If $\Lambda \in \mathfrak{b}_{\mathbb{C}}^{*}$ then by $I_{\Lambda}$ we denote the kernel of $\gamma_{\mathfrak{b}}(\cdot: \Lambda)$ in $\mathbb{D}(\mathrm{X})$. We denote by $\mathcal{C}(\mathrm{X}: \Lambda)$ the space of $L^{2}$-Schwartz functions on $\mathrm{X}$ annihilated by $I_{\Lambda}$. If $\mathcal{C}(\mathrm{X}: \Lambda)$ is non-trivial, then it contains a non-trivial $K$-finite function $f$. By a well known result of Harish-Chandra, the closed $G$-span of $f$ in $L^{2}(\mathrm{X})$ is a subrepresentation of finite length; see [41, p. 312, Thm. 12 and 42, p. 112, Thm. 4.2.1. Therefore, the mentioned closed $G$-span is contained in $L_{d}^{2}(\mathrm{X})$ and we deduce that $\Lambda \in \mathrm{L}(\mathrm{X}, \mathfrak{b})$. Conversely, if $\Lambda \in \mathrm{L}(\mathrm{X}, \mathfrak{b})$, then there exists a non-trivial $K$-finite function $f \in L_{d}^{2}(\mathrm{X})$ that is annihilated by $I_{\Lambda}$. From [3], Thm. 7.3, it follows that $f$ belongs to $\mathcal{C}(\mathrm{X}: \Lambda)$ and we see that the latter space is non-trivial. We conclude that

$$
\mathrm{L}(\mathrm{X}, \mathfrak{b})=\left\{\Lambda \in \mathfrak{b}_{\mathbb{C}}^{*} \mid \mathcal{C}(\mathrm{X}: \Lambda) \neq 0\right\} .
$$

Theorem 16.1 Assume that the space $L_{d}^{2}(\mathrm{X})$ is non-trivial. Then there exists a compact Cartan subspace $\mathfrak{t} \subset \mathfrak{q}$. Moreover, each $\Lambda \in \mathrm{L}(\mathrm{X}, \mathfrak{t})$ belongs to $i \mathfrak{t}^{*}$ and is regular with respect to $\Sigma(\mathfrak{t})$.

Remark 16.2 This result, which plays a crucial role in the description of the constant term of the normalized Eisenstein integral in Section 17 is essentially due to T. Oshima and M. Matsuki, 40. However, we have to be a bit careful here, since in our situation $G$ is assumed to be of Harish-Chandra's class, whereas in [40] it is assumed that $G$ is semisimple.

Proof: Fix a Cartan subspace $\mathfrak{b} \subset \mathfrak{q}$ that is fundamental, i.e., its compact part $\mathfrak{b}_{\mathrm{k}}=\mathfrak{b} \cap \mathfrak{k}$ is of maximal dimension. Then the assumption that $L_{d}^{2}(\mathrm{X})$ is nontrivial is equivalent to the assumption that $\mathrm{L}(\mathrm{X}, \mathfrak{b})$ is non-empty. We must show 
that under this assumption $\mathfrak{b}$ is compact, and all elements of $\mathrm{L}(\mathrm{X}, \mathfrak{b})$ belong to $i \mathfrak{b}^{*}$ and are regular.

Let $\mathrm{X}^{\circ}=G_{e} / G_{e} \cap H$ be the connected component of the origin in X. If $\Lambda \in \mathfrak{b}_{\mathbb{C}}^{*}$ then restriction defines a linear map $r: \mathcal{C}(\mathrm{X}: \Lambda) \rightarrow \mathcal{C}\left(\mathrm{X}^{\circ}: \Lambda\right)$. Conversely, extension by zero defines a linear embedding $j: \mathcal{C}\left(\mathrm{X}^{\circ}: \Lambda\right) \rightarrow \mathcal{C}(\mathrm{X}: \Lambda)$. Now $r \circ j=I$, hence $r$ is surjective. If the space $\mathcal{C}(\mathrm{X}: \Lambda)$ is non-trivial, then by $G$-invariance it follows that $r$ is non-zero, hence its image is non-trivial. On the other hand, if $\mathcal{C}\left(\mathrm{X}^{\circ}: \Lambda\right)$ is non-trivial, then $\mathcal{C}(\mathrm{X}: \Lambda)$ is non-trivial, by injectivity of $j$. Thus, from (16.1) we see that $\mathrm{L}(\mathrm{X}, \mathfrak{b})=\mathrm{L}\left(\mathrm{X}^{\circ}, \mathfrak{b}\right)$. Therefore, we may as well assume that $G$ is connected.

Let $\mathfrak{a}_{\Delta \mathrm{q}}$ be the intersection in $\mathfrak{a}_{\mathrm{q}}$ of the root spaces ker $\alpha, \alpha \in \Sigma$. This space is central in $\mathfrak{g}$. Hence, $\mathfrak{a}_{\Delta \mathfrak{q}} \subset \mathfrak{b}$ and the group $A_{\Delta q}:=\exp \mathfrak{a}_{\Delta q}$ is central in $G$.

The algebra $U\left(\mathfrak{a}_{\Delta q}\right)$ naturally embeds into $\mathbb{D}(\mathrm{X})$ and into $I(\mathfrak{b})$; accordingly, $\gamma$ restricts to the identity on $U\left(\mathfrak{a}_{\Delta \mathrm{q}}\right)$. Let $\Lambda \in \mathrm{L}(\mathrm{X}, \mathfrak{b})$ and let $f$ be a non-trivial function in $\mathcal{C}(\mathrm{X}: \Lambda)$. Then it follows that $R_{X} f=\Lambda(X) f$ for all $X \in \mathfrak{a}_{\Delta \mathrm{q}}$. Let $\Lambda_{0}:=\Lambda \mid \mathfrak{a}_{\Delta \mathrm{q}}$. Then it follows that $f(a x)=a^{\Lambda_{0}} f(x)$ for all $x \in \mathrm{X}$ and $a \in A_{\Delta \mathrm{q}}$. Since $f$ is a non-trivial Schwartz function, this implies that $\mathfrak{a}_{\Delta \mathrm{q}}=0$.

Let $\mathfrak{c}$ be the center of $\mathfrak{g}$. Then it follows that $\mathfrak{c}_{\mathrm{q}}:=\mathfrak{c} \cap \mathfrak{q}$ is contained in $\mathfrak{b} \cap \mathfrak{k}$. Let $\mathfrak{g}_{1}:=[\mathfrak{g}, \mathfrak{g}]$. Then $\mathfrak{b}=\mathfrak{c}_{\mathrm{q}} \oplus \mathfrak{b}_{1}$, with $\mathfrak{b}_{1}=\mathfrak{b} \cap \mathfrak{g}_{1}$. Accordingly, $I(\mathfrak{b})=U\left(\mathfrak{c}_{\mathrm{q}}\right) \otimes I\left(\mathfrak{b}_{1}\right)$.

Let $G_{1}$ be the analytic subgroup of $G$ with Lie algebra $\mathfrak{g}_{1}$ and let $H_{1}=$ $G_{1} \cap H$. The embeddings $\mathfrak{c}_{\mathrm{q}} \subset \mathfrak{g}$ and $\mathfrak{g}_{1} \subset \mathfrak{g}$ induce embeddings $U\left(\mathfrak{c}_{\mathrm{q}}\right) \subset \mathbb{D}(\mathrm{X})$ and $\mathbb{D}\left(G_{1} / H_{1}\right) \subset \mathbb{D}(\mathrm{X})$, via which we identify. Accordingly, $\mathbb{D}(\mathrm{X})=U\left(\mathfrak{c}_{\mathrm{q}}\right) \otimes$ $\mathbb{D}\left(G_{1} / H_{1}\right)$; moreover, the map $\gamma: \mathbb{D}(\mathrm{X}) \rightarrow I(\mathfrak{b})$ corresponds with the tensor product of $I_{U\left(\mathfrak{c}_{\mathrm{q}}\right)}$ and $\gamma_{1}$, the Harish-Chandra isomorphism for $\left(G_{1}, H_{1}, \mathfrak{b}_{1}\right)$.

If $\Lambda \in \mathrm{L}(\mathrm{X}, \mathfrak{b})$, let $\Lambda_{\mathfrak{c}}:=\left.\Lambda\right|_{\mathfrak{c}_{\mathrm{q}}}$ and $\Lambda_{1}:=\left.\Lambda\right|_{\mathfrak{b}_{1}}$. Then $U\left(\mathfrak{c}_{\mathrm{q}}\right) \simeq S\left(\mathfrak{c}_{\mathrm{q}}\right)$ acts by the character $\Lambda_{\mathfrak{c}}$ on the non-trivial space $\mathcal{C}(\mathrm{X}: \Lambda)$. This character must therefore be an infinitesimal character of the compact group $\exp \left(\mathfrak{c}_{\mathrm{q}}\right)$, hence belongs to $i \mathfrak{c}_{\mathrm{q}}^{*}$. On the other hand, $\mathbb{D}\left(G_{1} / H_{1}\right)$ acts by the character $\gamma_{1}\left(\cdot: \Lambda_{1}\right)$ on $\mathcal{C}(\mathrm{X}: \Lambda)$. Restriction to $G_{1} / H_{1}$ therefore induces a map $\mathcal{C}(\mathrm{X}: \Lambda) \rightarrow \mathcal{C}\left(G_{1} / H_{1}, \Lambda_{1}\right)$, which is non-zero by $G$-invariance and non-triviality of the space $\mathcal{C}(\mathrm{X}: \Lambda)$. Hence, $\Lambda_{1} \in \mathrm{L}\left(G_{1} / H_{1}, \mathfrak{b}_{1}\right)$. If $\mathfrak{b}_{1}$ is contained in $\mathfrak{k}$, then so is $\mathfrak{b}$ and if $\Lambda_{1} \in i \mathfrak{b}_{1}^{*}$ then $\Lambda=\Lambda_{\mathfrak{c}}+\Lambda_{1} \in i \mathfrak{b}^{*}$; finally, if $\Lambda_{1}$ is regular, then so is $\Lambda$. Therefore, we may as well assume that $\mathfrak{g}$ is semisimple from the start.

Let $Z(G)$ denote the center of $G$ and put $Z_{H}:=Z(G) \cap H$. Since $Z_{H}$ is discrete and central, ' $G:=G / Z_{H}$ is a Lie group with algebra naturally isomorphic with $\mathfrak{g}$. The involution $\sigma$ factors to an involution ' $\sigma$ of ' $G$. Moreover, $' H:=H / Z_{H}$, viewed as a subgroup of ' $G$, is an open subgroup of ' $G^{\prime} \sigma$. The associated symmetric space ${ }^{\prime} \mathrm{X}:={ }^{\prime} G /{ }^{\prime} H$ is naturally diffeomorphic with $\mathrm{X}$ and it is readily seen that $\mathrm{L}(\mathrm{X}, \mathfrak{b})=\mathrm{L}\left({ }^{\prime} \mathrm{X}, \mathfrak{b}\right)$. Therefore, it suffices to prove the assertions for ' $\mathrm{X}$ and we see that we may as well assume from the start that $Z_{H}=\{e\}$.

From now on we assume that $G$ is connected and semisimple, and that $Z_{H}=\{e\}$. The natural map $\pi: G / H_{e} \rightarrow G / H$ is a finite covering, hence induces a linear embedding $\pi^{*}: \mathcal{C}(G / H) \rightarrow \mathcal{C}\left(G / H_{e}\right)$ by pull-back. Via the isomorphism (2.5) we may identify the algebras $\mathbb{D}(G / H)$ and $\mathbb{D}\left(G / H_{e}\right)$, so that $\pi^{*}(D f)=$ $D \pi^{*} f$, for $f \in \mathcal{C}(G / H)$. Thus, if $\Lambda \in \mathrm{L}(\mathrm{X}, \mathfrak{b})$, then the image of $\mathcal{C}(G / H: \Lambda)$ 
in $\mathcal{C}\left(G / H_{e}\right)$ is a non-trivial subspace annihilated by the ideal $I_{\Lambda}$, from which we see that $\Lambda \in \mathrm{L}\left(G / H_{e}, \mathfrak{b}\right)$. It follows that we may as well assume that $H$ is connected. We will do so from now on.

Let $\mathfrak{g}^{d}$ be the dual real form of $\mathfrak{g}_{\mathbb{C}}$ defined as in Section 2 Via ad we identify $\mathfrak{g}_{\mathbb{C}}$ with the Lie algebra of the complex adjoint group $G_{\mathbb{C}}$ of $G$; accordingly, we denote by $G^{d}, K^{d}$ and $H^{d}$ the analytic subgroups of $G_{\mathbb{C}}$ with Lie algebras $\mathfrak{g}^{d}, \mathfrak{k}^{d}$ and $\mathfrak{h}^{d}$, respectively. Via Ad we may identify $K \cap H$ with a connected subgroup of $G_{\mathbb{C}}$. Accordingly, the map $(k, X) \mapsto k \exp X$ is a diffeomorphism from $(K \cap H) \times i[\mathfrak{k} \cap \mathfrak{q}]$ onto $H^{d}$. Hence, for every finite dimensional representation $(\pi, V)$ of $K$ there exists a unique finite dimensional representation $\left(\pi^{d}, V\right)$ of $H^{d}$ such that the infinitesimal representations associated with $\pi$ and $\pi^{d}$ have the same complex linear extension to $\mathfrak{k}_{\mathbb{C}}$. It follows that Flensted-Jensen's dualization procedure, see [25], Thm. 2.3, defines an injective linear map $f \mapsto f^{d}$ (denoted $f \mapsto f^{\eta}$ in 25] from the space $C^{\infty}(G / H)_{K}$ of $K$-finite smooth functions on $G / H$ into the space $C^{\infty}\left(G^{d} / K^{d}\right)_{H^{d}}$ of $H^{d}$-finite smooth functions on $G^{d} / K^{d}$. The map is determined by the property that, for every $f \in C^{\infty}(G / H)_{K}$ and all $u \in U\left(\mathfrak{k}_{\mathbb{C}}\right)$,

$$
\left.L_{u} f\right|_{A_{\mathrm{q}}}=\left.L_{u} f^{d}\right|_{A_{\mathrm{q}}} .
$$

We note that the left $H^{d}$-types of $f^{d}$ are all of the form $\pi^{d}$, with $\pi$ a finite dimensional irreducible representation of $K$. We also note that for $f \in C^{\infty}(G / H)_{K}$, the condition $f \in L^{2}(G / H)$ can be entirely rephrased in terms of the function $f^{d}$; in fact it is equivalent to the condition that $\left.L_{u} f^{d}\right|_{A_{\mathrm{q}}} \in L^{2}\left(A_{\mathrm{q}}, J d a\right)$, for all $u \in U(\mathfrak{k})$, with $J$ the Jacobian associated with the decomposition $G=K A_{\mathrm{q}} H$, see $[10,(3.1)$.

Let $D \mapsto D^{d}$ denote the natural algebra isomorphism from $\mathbb{D}(\mathrm{X})$ onto $\mathbb{D}\left(\mathrm{X}^{d}\right)$, corresponding to (2.5). Then $(D f)^{d}=D^{d} f^{d}$, for every $f \in C^{\infty}(G / H)_{K}$. Moreover, we recall from the text after (2.5) that $D^{d}=\gamma_{\mathfrak{a}_{\mathfrak{p}}^{d}}{ }^{-1} \circ \gamma_{\mathfrak{b}}(D)$, where we have written $\mathfrak{a}_{\mathfrak{p}}^{d}$ for the maximal abelian subspace ${ }^{d} \mathfrak{b}=\mathfrak{b}_{\mathbb{C}} \cap \mathfrak{g}^{d}$ of $\mathfrak{p}^{d}$. Now assume that $\Lambda \in \mathrm{L}(\mathrm{X}, \mathfrak{b})$. Then there exists a non-trivial $K$-finite function $f \in \mathcal{C}(\mathrm{X}: \Lambda)$. It follows that $f^{d} \in C^{\infty}\left(G^{d} / K^{d}\right)_{H^{d}}$ satisfies the system of differential equations $D f^{d}=\gamma_{\mathfrak{a}_{\mathfrak{p}}^{d}}(D: \Lambda) f^{d}$, for $D \in \mathbb{D}\left(G^{d} / K^{d}\right)$.

It follows from the above discussion, that the theorem of [40], p. 359, as well as its proof, can be entirely formulated in terms of the function $f^{d}$, and therefore applies without change, see 40, p. 388, note (i) added in proof. In particular, we may draw the following conclusions. In the notation of the cited theorem, we may take $\mathfrak{a}_{\mathfrak{p}}^{d}$ as above, and we may select a positive system $\Sigma\left(\mathfrak{a}_{\mathfrak{p}}^{d}\right)^{+}$ for $\Sigma\left(\mathfrak{g}^{d}, \mathfrak{a}_{\mathfrak{p}}^{d}\right)$ such that $\operatorname{Re} \Lambda$ is dominant. The hypothesis of part (i) of the cited theorem is fulfilled, since the non-trivial function $f$ belongs to the space $\mathcal{A}_{K}\left(G / H, \mathcal{M}_{\lambda}\right) \cap L^{2}(G / H)$, with $\lambda=\Lambda$. It follows that $\mathfrak{b}$ is compact, i.e., is contained in $\mathfrak{k} \cap \mathfrak{q}$. In the cited theorem we may now take $\mathfrak{t}=\mathfrak{b}$ and $\mathfrak{a}_{\mathfrak{p}}^{\prime}=i \mathfrak{b}$. Thus, $\mathfrak{a}_{\mathfrak{p}}^{d}=\mathfrak{a}_{\mathfrak{p}}^{\prime}$, and it follows from part (i) of the cited theorem that $\Lambda$ is regular.

We note that $W\left(\mathfrak{a}_{\mathfrak{p}}^{\prime} \mid \mathfrak{a}_{\mathfrak{p}}^{d}\right)=W\left(\mathfrak{a}_{\mathfrak{p}}^{d}\right)$, so that the elements $\bar{x}_{j}=\left.\operatorname{Ad}\left(x_{j}\right)\right|_{\mathfrak{a}_{\mathfrak{p}}^{d}}$ of the cited theorem belong to $W\left(\mathfrak{a}_{\mathfrak{p}}^{d}\right)$. It follows from part (iii) of the cited theorem that, for some $j$, the element $\bar{x}_{j} \Lambda=\lambda^{j}$ belongs to $\mathfrak{a}_{\mathfrak{p}}^{d *}$. This implies 
that $\Lambda \in \mathfrak{a}_{\mathfrak{p}}^{d *}=i \mathfrak{b}^{*}$.

Corollary 16.3 Let $\mathfrak{b} \subset \mathfrak{q}$ be a $\theta$-stable Cartan subspace. If $\mathrm{L}(\mathrm{X}, \mathfrak{b}) \neq \emptyset$ then there exists a Cartan subspace $\mathfrak{t} \subset \mathfrak{q}$ with $\mathfrak{t} \subset \mathfrak{k}$. Moreover, let $t$ be an element of the set $W(\mathfrak{t} \mid \mathfrak{b})$, which is non-empty by Lemma 3.8. Then, for every $\Lambda \in \mathrm{L}(\mathrm{X}, \mathfrak{b})$, the element $t \Lambda$ belongs to $i \mathrm{t}^{*}$ and is regular relative to the root system $\Sigma(\mathfrak{t})$.

Proof: Assume that $\mathrm{L}(\mathrm{X}, \mathfrak{b}) \neq \emptyset$. Then, by definition, $L_{\mathrm{d}}^{2}(\mathrm{X}) \neq 0$. By Theorem 16.1 there exists a compact Cartan subspace $\mathfrak{t} \subset \mathfrak{q}$. Let $t \in W(\mathfrak{b}, \mathfrak{t})$. Then by Lemma 14.2 the element $t$ maps $\mathrm{L}(\mathrm{X}, \mathfrak{t})$ bijectively onto $\mathrm{L}(\mathrm{X}, \mathfrak{b})$. The assertion now follows from Theorem 16.1

In the rest of this section we fix a Cartan subspace $\mathfrak{b} \subset \mathfrak{q}$ containing $\mathfrak{a}_{\mathrm{q}}$. If $P \in \mathcal{P}_{\sigma}$, then the $\theta$-stable Cartan subspace ${ }^{*} \mathfrak{b}_{P}$ of $\mathfrak{m}_{P} \cap \mathfrak{q}$ is defined as in the text before (2.6).

Lemma 16.4 Let $P \in \mathcal{P}_{\sigma}, v \in N_{K}\left(\mathfrak{a}_{\mathrm{q}}\right)$ and assume that $\mathrm{L}_{P, v}(\mathfrak{b}, \tau) \neq \emptyset$. Then there exist a Cartan subspace $\hat{\mathfrak{b}} \subset \mathfrak{m}_{1 v^{-1} P v} \cap \mathfrak{q}$ and an element $t \in W(\hat{\mathfrak{b}} \mid \mathfrak{b})$ with the following properties.

(a) ${ }^{*} \hat{\mathfrak{b}}:=\hat{\mathfrak{b}} \cap \mathfrak{m}_{v^{-1} P v}$ is compact, i.e., contained in $\mathfrak{k}$;

(b) $t=\operatorname{Ad}(v)^{-1}$ on $\mathfrak{a}_{P q}$;

(c) the elements of $t \mathrm{~L}_{P, v}(\mathfrak{b}, \tau)$ belong to $i^{*} \hat{\mathfrak{b}}^{*}$ and are regular relative to $\Sigma\left(\mathfrak{m}_{v^{-1} P v \mathbb{C}},{ }^{*} \hat{\mathfrak{b}}\right)$.

Proof: From (14.9) it follows that $\mathrm{L}\left(\mathrm{X}_{P, v},{ }^{*} \mathfrak{b}_{P, v}, \tau_{P}\right) \neq \emptyset$. Hence, by Corollary 16.3 there exists a Cartan subspace $\mathfrak{t}$ of $\mathfrak{m}_{P} \cap \operatorname{Ad}(v)(\mathfrak{q})$ that is contained in $\mathfrak{k}$. Now $\hat{\mathfrak{b}}=\operatorname{Ad}(v)^{-1}\left(\mathfrak{t} \oplus \mathfrak{a}_{P \mathfrak{q}}\right)$ is a Cartan subspace of $\mathfrak{m}_{1 v^{-1} P v} \cap \mathfrak{q}$ that satisfies condition (a), with ${ }^{*} \hat{\mathfrak{b}}=\operatorname{Ad}(v)^{-1} \mathfrak{t}$.

Fix $t_{1} \in W(\hat{\mathfrak{b}} \mid \mathfrak{b})$. Then $t_{1}^{-1} \operatorname{Ad}(v)^{-1} \mathfrak{a}_{P \mathfrak{q}} \subset{ }^{\mathrm{d}} \mathfrak{b}$. Since ${ }^{\mathrm{d}} \mathfrak{b}$ is maximal abelian in $\mathfrak{p}^{d}$, it follows from Lemma 3.1 (d) that there exists a $t_{2} \in W(\mathfrak{b})$ such that $t_{2}=t_{1}^{-1} \operatorname{Ad}(v)^{-1}$ on $\mathfrak{a}_{P \mathfrak{q}}$. It follows that $t=t_{1} t_{2} \in W(\hat{\mathfrak{b}} \mid \mathfrak{b})$ satisfies requirement (b).

Finally, let $\Lambda \in \mathrm{L}_{P, v}(\mathfrak{b}, \tau)$. Then, in the notation of (14.9), the element $\Lambda^{\prime}:=$ $\operatorname{Ad}(v) s^{-1} \Lambda$ belongs to $\mathrm{L}\left(\mathrm{X}_{P, v},{ }^{*} \mathfrak{b}_{P, v}, \tau_{P}\right)$. The element $t^{\prime}=\operatorname{Ad}(v) t s \operatorname{Ad}(v)^{-1}$ belongs to $W\left(\hat{\mathfrak{b}}^{v} \mid \mathfrak{b}^{v}\right)$ and equals the identity on $\mathfrak{a}_{P \mathrm{q}}$, hence restricts to an element of $W\left(\mathfrak{t} \mid{ }^{*} \mathfrak{b}_{P, v}\right)$. By Corollary 16.3 it follows that $t^{\prime} \Lambda^{\prime}$ belongs to $i \mathfrak{t}^{*}$ and is regular relative to $\Sigma\left(\mathfrak{m}_{P \mathbb{C}}, \mathfrak{t}\right)$. We now observe that $t^{\prime} \Lambda^{\prime}=\operatorname{Ad}(v) t \Lambda$. Hence, $t \Lambda$ belongs to $i^{*} \hat{\mathfrak{b}}$ and is regular with respect to $\Sigma\left(\mathfrak{m}_{v^{-1} P v \mathfrak{C}},{ }^{*} \hat{\mathfrak{b}}\right)$.

Remark 16.5 Let $P, v, \hat{\mathfrak{b}}, t$ be as in Lemma 16.4 Then it follows from Lemma 3.8 that, for all $\Lambda \in \mathrm{L}_{P, v}(\mathfrak{b}, \tau), \nu \in \mathfrak{a}_{P \mathrm{qC}}^{*}$ and $D \in \mathbb{D}(\mathrm{X})$,

$$
\gamma_{\mathfrak{b}}(D: \Lambda+\nu)=\gamma_{\hat{\mathfrak{b}}}(D: t \Lambda+t \nu) .
$$


Corollary 16.6 Let $P \in \mathcal{P}_{\sigma}$ and let $\Lambda \in \mathrm{L}_{P}(\mathfrak{b}, \tau)$. Then $\langle\Lambda, \alpha\rangle \in \mathbb{R} \backslash\{0\}$, for each $\alpha \in \Sigma\left(\mathfrak{m}_{P \mathbb{C}},{ }^{*} \mathfrak{b}_{P}\right)$.

Proof: Select $v \in{ }^{P} \mathcal{W}$ such that $\Lambda \in \mathrm{L}_{P, v}(\mathfrak{b}, \tau)$. Let $\hat{\mathfrak{b}}, t$ be associated as in Lemma16.4 Then by (a) and (b) of the mentioned lemma, $t$ maps $\mathfrak{b}={ }^{*} \mathfrak{b}_{P} \oplus \mathfrak{a}_{P \mathrm{q}}$ onto $\hat{\mathfrak{b}}={ }^{*} \hat{\mathfrak{b}} \oplus \mathfrak{a}_{v^{-1} P v \mathfrak{q}}$, preserving the decompositions. The assertion now follows from Lemma 16.4 (a) and (c).

\section{The constant term of the Eisenstein integral}

In this section we describe the constant term of the normalized Eisenstein integral, introduced in Definition 13.7. We start by recalling the notion of the constant term introduced in 17 .

If $f \in \mathcal{A}_{\text {temp }}(\mathrm{X}: \tau)$, see Def. 10.1 then in particular $f \in \mathcal{A}\left(\mathrm{X}_{+}: \tau\right)$ and $f$ has an expansion of the form (6.1). It follows from Lemma 10.2 (c) combined with [14, Thm. 3.5, that, for each $Q \in \mathcal{P}_{\sigma}$ and every $v \in N_{K}\left(\mathfrak{a}_{\mathrm{q}}\right)$,

$$
\xi \in \operatorname{Exp}(Q, v \mid f) \Rightarrow \operatorname{Re} \xi+\rho_{Q} \leq 0 \quad \text { on } \quad \mathfrak{a}_{Q \mathrm{q}}^{+} .
$$

We define the function $f_{Q, v}: \mathrm{X}_{1 Q, v,+} \rightarrow V_{\tau}$ by

$$
f_{Q, v}(m a)=d_{Q}(m a) \sum_{\substack{\xi \in \operatorname{Exp}(Q, v \mid f) \\ \operatorname{Re} \xi+\rho Q=0}} a^{\xi} q_{\xi}(Q, v \mid f, \log a, m),
$$

for $m \in \mathrm{X}_{Q, v,+}, a \in A_{Q \mathrm{q}}$. Here $d_{Q}: M_{1 Q} \rightarrow \mathbb{R}$ is defined by $d_{Q}(m)=\sqrt{\left.|\operatorname{det} \operatorname{Ad}(m)|\right|_{\mathfrak{n}_{Q}}} \mid$. Note that $d_{Q}=1$ on $M_{Q}$ and on $A_{Q} \cap H$. Hence, $d_{Q}$ factors to a function on $\mathrm{X}_{1 Q, v}$; in fact, $d_{Q}(m a)=a^{\rho_{Q}}$, for $m \in M_{Q \sigma}$ and $a \in A_{Q \mathrm{q}}$.

We note that, for $v \in N_{K}\left(\mathfrak{a}_{\mathrm{q}}\right)$, the function $R_{v} f: x \mapsto f(x v)$ belongs to the space $\mathcal{A}_{\text {temp }}\left(G / v H v^{-1}: \tau\right)$.

\section{Proposition 17.1}

(a) If $u, v \in N_{K}\left(\mathfrak{a}_{\mathrm{q}}\right)$, then $\left(R_{v} f\right)_{Q, u}=f_{Q, u v}$.

(b) The function $f_{Q, v}$ extends uniquely to smooth function on $\mathrm{X}_{1 Q, v}$. This extension is the unique function in $\mathcal{A}_{\mathrm{temp}}\left(\mathrm{X}_{1 Q, v}: \tau_{Q}\right)$ such that

$$
\lim _{t \rightarrow \infty}\left(d_{Q}(m \exp t X) f(m \exp t X v)-f_{Q, v}(m \exp t X)\right)=0,
$$

for every $m \in M_{1 Q}$ and $X \in \mathfrak{a}_{Q q}^{+}$.

Proof: The first assertion follows from [14, Lemma 3.7. In view of (a) it suffices to prove the second assertion for $v=e$. In this case the assertion follows from [17, proof of Thm. 1. 
Thus, for $v=e$, the function $f_{Q, v}$ coincides with the constant term of $f$ along $Q$, introduced by [17, which in turn generalizes Harish-Chandra's notion of the constant term for the case of the group, see [30], Sect. 21, Thm. 1. We shall therefore call $f_{Q, v}$ the constant term of $f$ along $(Q, v)$. The following result, which generalizes a result of Harish-Chandra, see [30, Sect. 21, Lemma 1, is essentially given in [17, Thm. 1 (b).

Lemma 17.2 (Transitivity of the constant term) Let $P, Q \in \mathcal{P}_{\sigma}$ be such that $P \subset Q$. Put ${ }^{* 1} P:=M_{1 Q} \cap P$. Let $v \in N_{K}\left(\mathfrak{a}_{\mathrm{q}}\right)$ and $u \in N_{K_{Q}}\left(\mathfrak{a}_{\mathrm{q}}\right)$. Then

$$
\left(f_{Q, v}\right)_{* 1}^{* 1, u}=f_{P, u v} .
$$

Proof: For $v=u=e$ the result is equivalent to [17, Thm. 1(b). Let now $v \in$ $N_{K}\left(\mathfrak{a}_{\mathrm{q}}\right)$ and $u \in N_{K_{Q}}\left(\mathfrak{a}_{\mathrm{q}}\right)$ be general. Then right translation by $u$ defines a linear isomorphism $R_{u}: \mathcal{A}\left(\mathrm{X}_{1 Q, v}: \tau_{Q}\right) \rightarrow \mathcal{A}\left(\mathrm{X}_{1 Q, u v}: \tau_{Q}\right)$. Hence, applying Proposition 17.1 (b) we find that

$$
R_{u}\left(f_{Q, v}\right)=f_{Q, u v} .
$$

Applying Proposition 17.1 (a) we see that

$$
f_{Q, u v}=\left(R_{u v} f\right)_{Q, e} \quad \text { and } \quad\left(f_{Q, v}\right)_{* 1} P, u=\left(R_{u} f_{Q, v}\right)_{* 1} P, e .
$$

Combining (17.1) with (17.2), and using the first line of the proof and Proposition 17.1 (a), we finally obtain that

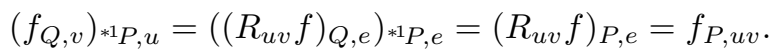

The following transformation rule for the constant term will also be useful to us. If $u, v \in N_{K}\left(\mathfrak{a}_{\mathrm{q}}\right)$, we define the map $\rho_{\tau, u}: C^{\infty}\left(\mathrm{X}_{1 Q, v}: \tau_{Q}\right) \rightarrow C^{\infty}\left(\mathrm{X}_{1 u Q u^{-1}, u v}: \tau_{u Q u^{-1}}\right)$ in accordance with [14, Eqn. (3.24), by

$$
\rho_{\tau, u} \varphi(m)=\tau(u) \varphi\left(u^{-1} m u\right), \quad\left(m \in M_{1 u Q u^{-1}}\right) .
$$

One readily checks that $\rho_{\tau, u}$ maps $\mathcal{A}_{\text {temp }}\left(\mathrm{X}_{1 Q, v}: \tau_{Q}\right)$ into $\mathcal{A}_{\text {temp }}\left(\mathrm{X}_{1 u Q u^{-1}, u v}: \tau_{u Q u^{-1}}\right)$.

Lemma 17.3 Let $f \in \mathcal{A}_{\text {temp }}(\mathrm{X}: \tau)$ and let $Q \in \mathcal{P}_{\sigma}$ and $u, v \in N_{K}\left(\mathfrak{a}_{\mathrm{q}}\right)$. Then

$$
f_{u Q u^{-1}, u v}=\rho_{\tau, u} f_{Q, v} .
$$

Proof: From the definition of $d_{Q}$ one readily verifies that $d_{u Q u^{-1}}\left(u m u^{-1}\right)=$ $d_{Q}(m)$, for $m \in M_{1 Q}$. The result now follows by a straightforward application of Proposition 17.1 (b). See [14, Lemma 3.6, for a similar proof.

Assume that $\Omega \subset i \mathfrak{a}_{P \mathrm{q}}^{*}$ is open, and $f: \Omega \times \mathrm{X} \rightarrow V_{\tau}$ a smooth map such that $f_{\nu}: x \mapsto f(\nu, x)$ belongs to $\mathcal{A}_{\text {temp }}(\mathrm{X}: \tau)$ for every $\nu \in \Omega$. If $Q \in \mathcal{P}_{\sigma}$ and $v \in N_{K}\left(\mathfrak{a}_{\mathrm{q}}\right)$, we shall write $f_{Q, v}$ for the map $\Omega \times \mathrm{X}_{1 Q, v} \rightarrow V_{\tau}$ defined by

$$
f_{Q, v}(\nu, m)=\left(f_{\nu}\right)_{Q, v}(m), \quad\left(\nu \in \Omega, m \in \mathrm{X}_{1 Q, v}\right) .
$$


We now turn our attention to the normalized Eisenstein integral $E^{\circ}(P: \cdot)$ where $P \in \mathcal{P}_{\sigma}$ is assumed to be of residue type. In the end it will follow that any $P \in \mathcal{P}_{\sigma}$ is of this type, see Remark [2.2, so that this is really no restriction on $P$. Let $\Omega_{P}$ be the set of points in $i \mathfrak{a}_{P q}^{*}$ where the function $\nu \mapsto E^{\circ}(P: \nu)$ is regular. Then for $\nu \in \Omega_{P}$ and $\psi \in \mathcal{A}_{2, P}$, the function $E^{\circ}(P: \nu: \cdot) \psi$ belongs to $\mathcal{A}_{\text {temp }}(\mathrm{X}: \tau)$, see Proposition 13.15. In accordance with the above, we denote its constant term along $(Q, v)$, for $Q \in \mathcal{P}_{\sigma}$ and $v \in N_{K}\left(\mathfrak{a}_{\mathrm{q}}\right)$, by $E_{Q, v}^{\circ}(P: \nu: \cdot) \psi$.

Proposition $17.4(\mathrm{RT})$ Let $P, Q \in \mathcal{P}_{\sigma}$ and $u \in N_{K}\left(\mathfrak{a}_{\mathrm{q}}\right)$.

(a) The function $E_{Q, u}^{\circ}(P: \cdot)$ extends to a meromorphic $C^{\infty}\left(\mathrm{X}_{1 Q, u}, \operatorname{Hom}\left(\mathcal{A}_{2, P}, V_{\tau}\right)\right)$ valued function on $\mathfrak{a}_{P \mathrm{qC}}^{*}$, with singular set equal to a locally finite union of real $\Sigma_{r}(P)$-hyperplanes.

(b) There exists a $\varepsilon>0$ such that, for every $\psi \in \mathcal{A}_{2, P}$ and $p \in \Pi_{\Sigma_{r}(P)}\left(\mathfrak{a}_{P \mathrm{q}}^{*}\right)$ with the property that $\nu \mapsto p(\nu) E^{\circ}(P: \nu: \cdot) \psi$ is regular on $\mathfrak{a}_{P \mathrm{q}}^{*}(\varepsilon)$, the function $\nu \mapsto p(\nu) E_{Q, u}^{\circ}(P: \nu: \cdot) \psi$ is regular on $\mathfrak{a}_{P \mathrm{q}}^{*}(\varepsilon)$ as well.

(c) If $E_{Q, u}^{\circ}(P: \cdot) \neq 0$, then $W\left(\mathfrak{a}_{P q} \mid \mathfrak{a}_{Q q}\right)$ is non-empty.

(d) Let $W\left(\mathfrak{a}_{P q} \mid \mathfrak{a}_{Q q}\right)$ be non-empty. Then there exist unique meromorphic functions $E_{Q, u, s}^{\circ}(P: \cdot): \mathfrak{a}_{P \mathrm{qC}}^{*} \rightarrow \operatorname{Hom}\left(\mathcal{A}_{2, P}, C^{\infty}\left(\mathrm{X}_{Q, u}: \tau_{Q}\right)\right)$, for $s \in$ $W\left(\mathfrak{a}_{P \mathrm{q}} \mid \mathfrak{a}_{Q \mathrm{q}}\right)$, such that, for all $m \in \mathrm{X}_{Q, u}$ and $a \in A_{Q \mathrm{q}}$,

$$
E_{Q, u}^{\circ}(P: \nu: m a)=\sum_{s \in W\left(\mathfrak{a}_{P \mathrm{q}} \mid \mathfrak{a}_{Q \mathrm{q}}\right)} a^{s^{*} \nu} E_{Q, u, s}^{\circ}(P: \nu: m),
$$

as an identity of meromorphic functions in the variable $\nu \in \mathfrak{a}_{Q \mathrm{qC}}^{*}$. Here $s^{*} \nu=\nu \circ s$, see $\S$ 3. The singular locus of any of the meromorphic functions $E_{Q, u, s}^{\circ}(P: \cdot)$, for $s \in W\left(\mathfrak{a}_{P \mathrm{q}} \mid \mathfrak{a}_{Q \mathrm{q}}\right)$, is the union of a locally finite collection of real $\Sigma_{r}(P)$-hyperplanes.

Proof: We give the proof under the assumption that $P$ is of residue type, see Remark 12.2

(a): Let $\psi \in \mathcal{A}_{2, P}$, and define $f:(\nu, x) \mapsto E^{\circ}(P: \nu: x) \psi$. Then $f \in$ $\mathcal{E}_{P, Y}^{\text {hyp }}(\mathrm{X}: \tau)$, with $Y \subset{ }^{*} \mathfrak{a}_{P \mathrm{q}}^{*}$ a finite subset, see Lemma 13.17 In particular, it follows that $f \in C_{P, Y}^{\mathrm{ep}, \mathrm{hyp}}\left(\mathrm{X}_{+}: \tau\right)$, see Section [6] The set $\mathcal{H}:=\mathcal{H}_{f}$ is a real $\Sigma_{r}(P)$-configuration in $\mathfrak{a}_{P \mathrm{qC}}^{*}$, again by Lemma 13.17

Let $\mathcal{H}_{0}$ be the collection of $H \in \mathcal{H}$ with $H \cap i \mathfrak{a}_{P \mathrm{q}}^{*} \neq \emptyset$. Then $\mathcal{H}_{0}$ is finite, since $\mathcal{H}$ is real. For every $H \in \mathcal{H}_{0}$ we select a first degree polynomial function $l_{H} \in P_{1}\left(\mathfrak{a}_{P \mathrm{q}}^{*}\right)$ with $H=l_{H}^{-1}(0)$, and put

$$
\pi_{0}=\prod_{H \in \mathcal{H}_{0}} l_{H}^{d(H)}
$$

with $d=d_{f}$. Select $\varepsilon_{0}>0$ such that $H \in \mathcal{H}, H \cap \mathfrak{a}_{P q}^{*}\left(\varepsilon_{0}\right) \neq \emptyset \Rightarrow H \in \mathcal{H}_{0}$. Then the family $f^{0}:(\nu, x) \mapsto \pi_{0}(\nu) f(\nu, x)$ belongs to $\mathcal{O}\left(\mathfrak{a}_{P \mathrm{q}}^{*}\left(\varepsilon_{0}\right), C^{\infty}(\mathrm{X}: \tau)\right)$. 
Moreover, in view of Lemma 13.17 for every $\sigma \in W / \sim_{Q \mid P}$ and $\xi \in-\sigma \cdot Y+$ $\mathbb{N} \Delta_{r}(Q)$, the function

$$
q_{\sigma, \xi}^{0}(Q, u \mid f):=\pi_{0} q_{\sigma, \xi}(Q, u \mid f)
$$

belongs to $P_{k}\left(\mathfrak{a}_{Q \mathrm{q}}\right) \otimes \mathcal{O}\left(\mathfrak{a}_{P \mathrm{q}}^{*}\left(\varepsilon_{0}\right), C^{\infty}\left(\mathrm{X}_{Q, u}: \tau_{Q}\right)\right)$; here $k=\operatorname{deg}_{a} f$. It follows from 14, Lemma 12.7, that

$$
f_{\nu}^{0}(\mathrm{mau})=\sum_{\sigma \in W / \sim_{Q \mid P}} a^{\sigma \nu-\rho_{Q}} \sum_{\xi \in-\sigma \cdot Y+\mathbb{N} \Delta_{r}(Q)} a^{-\xi} q_{\sigma, \xi}^{0}(Q, u \mid f, \log a)(\nu, m),
$$

for every $m \in \mathrm{X}_{Q, u,+}$, and $a \in A_{Q \mathrm{q}}^{+}\left(R_{Q, u}(m)^{-1}\right)$, where the second series converges neatly in $a$. For every $\nu \in \Omega_{P}$, the function $f_{\nu}^{0}$ belongs to $\mathcal{A}_{\text {temp }}(\mathrm{X}: \tau)$, see Proposition 13.15. Since $Y$ is real, it follows by uniqueness of asymptotics, for all $m \in \mathrm{X}_{Q, u,+}$ and $a \in A_{Q q}$, that

$$
\left(f_{\nu}^{0}\right)_{Q, u}(m a)=\sum_{\substack{\sigma \in W / \sim_{Q \mid P} \\ 0 \in-\sigma \cdot Y+\mathbb{N}_{r}(Q)}} a^{\sigma \nu} q_{\sigma, 0}^{0}(Q, u \mid f, \log a)(\nu, m) .
$$

By density and continuity, this expression holds for all $m \in \mathrm{X}_{Q, u}$ and $a \in A_{Q \mathrm{q}}$. On the other hand, by the characterization of the constant term in Proposition 17.1 (b), it follows that, for $\nu \in \Omega_{P}$,

$$
\left(f_{\nu}^{0}\right)_{Q, u}=\pi_{0}(\nu)\left(f_{\nu}\right)_{Q, u} .
$$

Using Lemma 13.17 once again, we infer from (17.5) that $\nu \mapsto E_{Q, u}^{\circ}(P: \nu) \psi=$ $\left(f_{\nu}\right)_{Q, u}$ extends to a meromorphic $C^{\infty}\left(\mathrm{X}_{1 Q, u}: \tau_{Q}\right)$-valued function on $\mathfrak{a}_{P \text { qC }}^{*}$ with singular set contained in $\cup \mathcal{H}_{f}$. This establishes (a).

We will first establish the remaining assertions under the assumption that $u=e$.

(b): Let $\Lambda \in \mathrm{L}_{P}(\mathfrak{b}, \tau), \psi \in \mathcal{A}_{2, P}(\Lambda)$ and define $f$ as above. For $p \in$ $\Pi_{\Sigma_{r}(P)}\left(\mathfrak{a}_{P \mathrm{q}}^{*}\right)$ we put $f_{p}(\nu, x)=p(\nu) f(\nu, x)$.

According to Theorem 15.12 there exist $q \in \Pi_{\Sigma_{r}\left(\mathfrak{a}_{P q}^{*}\right), \mathbb{R}}\left(\mathfrak{a}_{P \mathrm{q}}^{*}\right)$ and constants $\varepsilon_{0}>0$ and $s_{0}>0$, all independent of $\Lambda$ and $\psi$, such that $f_{q}$ is holomorphic on $\mathfrak{a}_{P q}^{*}\left(\varepsilon_{0}\right)$ and belongs to $\mathcal{T}\left(P, \tau, \Lambda, \varepsilon_{0}, s_{0}\right)$. Let $\varepsilon_{1}$ be any constant with $0<$ $\varepsilon_{1}<\varepsilon_{0}$. If $p \in \Pi_{\Sigma_{r}(P)}\left(\mathfrak{a}_{P \mathrm{q}}^{*}\right)$ is such that $f_{p}$ is holomorphic on $\mathfrak{a}_{P \mathrm{q}}^{*}\left(\varepsilon_{1}\right)$, then clearly $f_{p q} \in \mathcal{T}\left(P, \tau, \Lambda, \varepsilon_{0}, s_{0}\right)$. By a repeated application of Cauchy's integral formula to $f_{p q}(\nu, x)$, with polydiscs of size $O\left(\left(1+l_{\mathrm{X}}(x)\right)^{-1}\right)$, it now follows that $f_{p} \in \mathcal{T}\left(P, \tau, \Lambda, \varepsilon_{1}^{\prime}, s_{0}\right)$, for every $\varepsilon_{1}^{\prime}$ with $0<\varepsilon_{1}^{\prime}<\varepsilon_{1}$. See [5], Lemma 6.1, for a more detailed indication of how to use Cauchy's formula.

Let $v \in{ }^{P} \mathcal{W}$ be such that $\Lambda \in \mathrm{L}_{P, v}(\mathfrak{b}, \tau)$, let $\hat{\mathfrak{b}}, t$ be as in Lemma 16.4 and put $\hat{P}=v^{-1} P v$. Then, in view of Remark 16.5 the family $\hat{f}_{p}: \mathfrak{a}_{\hat{P} \mathrm{qC}}^{*} \times \mathrm{X} \rightarrow V_{\tau}$ defined by $\hat{f}_{p}(\mu, x)=f_{p}\left(t^{-1} \mu, x\right)$ belongs to $\mathcal{T}\left(\hat{P}, \tau, t \Lambda, \varepsilon_{0}, s_{0}\right)$.

Since $t \Lambda \in i^{*} \hat{\mathfrak{b}}^{*}$, by Lemma 16.4 (c), we may apply [17, Thm. 3, which in turn is based on [5], Thm. 12.9. Let $\varepsilon_{1}^{\prime}<\varepsilon_{1}<\varepsilon_{0}$ be as above. According to the mentioned theorem there exists a constant $\bar{\varepsilon}_{1}^{\prime}>0$ such that for every 
$F \in \mathcal{T}\left(\hat{P}, \tau, t \Lambda, \varepsilon_{1}^{\prime}, s_{0}\right)$, and all $m \in \mathrm{X}_{1 Q, e}$, the function $\nu \mapsto\left(F_{\nu}\right)_{Q, e}$ is holomorphic on $\mathfrak{a}_{\hat{P} \mathfrak{q}}^{*}\left(\varepsilon_{1}^{\prime}\right)$. From the proof of [17], Thm. 3, it follows that this holds with $\bar{\varepsilon}_{1}^{\prime}=\min \left(\varepsilon_{1}^{\prime}, \bar{\varepsilon}\right)$, where $\bar{\varepsilon}>0$ is the constant of [17], Lemma 5 . The latter constant only depends on $\Lambda$; the set $\mathrm{L}_{P}(\mathfrak{b}, \tau)$ is finite, hence we may chose $\bar{\varepsilon}$ simultaneously for all $\Lambda$ under consideration. We now fix $\varepsilon>0$ such that $\varepsilon<\min \left(\varepsilon_{0}, \bar{\varepsilon}\right)$. Assume that the hypothesis of part (b) of the theorem is fulfilled. If we apply the above discussion to the functions $f_{p}$ and $F=\hat{f}_{p}$, with $\varepsilon_{1}=\varepsilon$, then $\bar{\varepsilon}_{1}^{\prime}=\varepsilon_{1}^{\prime}$, and it follows that the function $\nu \mapsto\left(\left(f_{p}\right)_{\nu}\right)_{Q, e}(m)=p(\nu) E_{Q, e}^{\circ}(P: \nu: m) \psi$ is holomorphic on $\mathfrak{a}_{P \mathrm{q}}^{*}\left(\varepsilon_{1}^{\prime}\right)$, for all $m \in \mathrm{X}_{Q, e}$. In view of part (a) of the theorem, it follows that $\nu \mapsto p(\nu) E_{Q, e}^{\circ}(P: \nu) \psi$ is holomorphic on $\mathfrak{a}_{P q}^{*}\left(\varepsilon_{1}^{\prime}\right)$ as a function with values in $C^{\infty}\left(\mathrm{X}_{1 Q, e}: \tau_{Q}\right)$. This holds for every $\varepsilon_{1}^{\prime}<\varepsilon$, whence the desired assertion.

(c): From the hypothesis with $v=e$ it follows that there exists a $\Lambda \in$ $\mathrm{L}_{P}(\mathfrak{b}, \tau)$ and a $\psi \in \mathcal{A}_{2, P}(\Lambda)$ such that $E_{Q, u}^{\circ}(P: \cdot) \psi \neq 0$. Let $\Omega_{P}^{\prime}$ be the set of $\nu \in \Omega_{P}$ such that $\Lambda+\nu$ is a $\Sigma(\mathfrak{b})$-regular element of $\mathfrak{b}_{\mathbb{C}}^{*}$. It follows from Lemma 16.4 that $\Lambda$ is regular with respect to $\Sigma\left(\mathfrak{m}_{P \mathbb{C}},{ }^{*} \mathfrak{b}_{P}\right)$. Therefore, $\Omega_{P}^{\prime}$ is open dense in $\Omega_{P}$, hence in $i \mathfrak{a}_{P q}^{*}$. We infer that we may select $\nu \in \Omega_{P}^{\prime}$ such that $\left(f_{\nu}\right)_{Q, e} \neq 0$, with notation as introduced in part (a) of this proof.

Fix $v \in{ }^{P} \mathcal{W}$ such that $\Lambda \in \mathrm{L}_{P, v}(\mathfrak{b}, \tau)$. Let $(\hat{\mathfrak{b}}, t)$ be as in Lemma 16.4 and put $\hat{P}:=v^{-1} P v$. Then $t \Lambda$ belongs to $i^{*} \hat{\mathfrak{b}}^{*}$ and is regular relative to $\Sigma\left(\mathfrak{m}_{\hat{P}_{\mathbb{C}}}{ }^{*} \hat{\mathfrak{b}}\right)$. Now $f_{\nu} \in \mathcal{A}_{\text {temp }}(\mathrm{X}: \tau)$ and by Remark 16.5

$$
D f_{\nu}=\gamma_{\hat{\mathfrak{b}}}(D: t \Lambda+t \nu) f_{\nu}
$$

for all $D \in \mathbb{D}(\mathrm{X})$. Since $t \Lambda \in i^{*} \hat{\mathfrak{b}}^{*}, t \nu \in i \mathfrak{a}_{\hat{P}_{\mathrm{q}}}$ and $t \Lambda+t \nu$ is regular with respect to $\Sigma(\hat{\mathfrak{b}})$, it follows from [17, Thm. 2, that the set $W\left(\mathfrak{a}_{\hat{P}_{\mathrm{q}}} \mid \mathfrak{a}_{Q \mathrm{q}}\right)$ is non-empty. The map $s \mapsto t^{-1} \circ s$ is a bijection from the latter set onto $W\left(\mathfrak{a}_{P q} \mid \mathfrak{a}_{Q q}\right)$, which set is therefore non-empty as well.

(d): Uniqueness of the functions $E_{Q, e, s}^{\circ}(P: \cdot)$ is obvious, by linear independence of the functions $a \mapsto a^{s^{*} \nu}$ for generic $\nu$. We fix $\Lambda$ and $\psi$ as in part (b) of the proof and define $f$ as in part (a). We define the set $\Omega_{P}^{\prime}$ as in (c). Let $\Omega_{P}^{\prime \prime}$ be the open dense subset consisting of $\nu \in \Omega_{P}^{\prime}$ with $s^{*} \nu$ mutually different, for $s \in W\left(\mathfrak{a}_{P \mathrm{q}} \mid \mathfrak{a}_{Q \mathrm{q}}\right)$. Let $v, \hat{\mathfrak{b}}, t, \hat{P}$ be as in part (c) of the proof, and fix $\nu \in \Omega_{P}^{\prime \prime}$. In view of (17.6), it follows from [17, Thm. 2, that there exists a collection of functions $f_{t \nu, \hat{s}, Q} \in C^{\infty}\left(\mathrm{X}_{1 Q, e}: \tau_{Q}\right)$, for $\hat{s} \in W\left(\mathfrak{a}_{\hat{P}_{\mathrm{q}}} \mid \mathfrak{a}_{Q \mathrm{q}}\right)$, such that

$$
\left(f_{\nu}\right)_{Q, e}(m)=\sum_{\hat{s} \in W\left(\mathfrak{a}_{\hat{P}_{q}} \mid \mathfrak{a}_{Q q}\right)} f_{t \nu, \hat{s}, Q}(m), \quad\left(m \in \mathrm{X}_{1 Q, e}\right) ;
$$

and

$$
f_{t \nu, \hat{s}, Q}(m a)=a^{\hat{s}^{*} t \nu} f_{t \nu, \hat{s}, Q}(m), \quad\left(m \in \mathrm{X}_{1 Q, e}, a \in A_{Q \mathrm{q}}\right) .
$$

Combining these equations, substituting $t s$ for $\hat{s}$ and writing $f_{\nu, s, Q}=f_{t \nu, t s, Q}$, we see that, for all $m \in \mathrm{X}_{Q, e}$ and $a \in A_{Q \mathrm{q}}$,

$$
\left(f_{\nu}\right)_{Q, e}(m a)=\sum_{s \in W\left(\mathfrak{a}_{P q} \mid \mathfrak{a}_{Q q}\right)} a^{s^{*} \nu} f_{\nu, s, Q}(m) .
$$


For every $s \in W\left(\mathfrak{a}_{P \mathrm{q}} \mid \mathfrak{a}_{Q \mathrm{q}}\right)$ there exists an element $\tilde{s} \in W$ such that $s=\left.\tilde{s}\right|_{\mathfrak{a}_{Q \mathrm{q}}}$, see Corollary 3.5 It follows that $s^{*} \nu=\left.\tilde{s}^{-1} \nu\right|_{\mathfrak{a}_{Q q}}$, for all $\nu \in \mathfrak{a}_{P \mathrm{qC}}^{*}$. Using the definition of $\sim_{Q \mid P}$ we see that the class of $\tilde{s}^{-1}$ in $W / \sim_{Q \mid P}$ is uniquely determined by $s$. We denote this class by $\sigma_{s}$. Comparing (17.5) and (17.7) we see by uniqueness of asymptotics that $X \mapsto q_{\sigma_{s}, 0}(Q, e \mid f, X)(\nu)$ is constant as a $C^{\infty}\left(\mathrm{X}_{Q, e}: \tau_{Q}\right)$-valued function on $\mathfrak{a}_{Q \mathrm{q}}$ and that

$$
f_{\nu, s, Q}(m)=q_{\sigma_{s}, 0}(Q, e \mid f, 0)(\nu, m),
$$

for all $m \in \mathrm{X}_{Q, e}$. We define $E_{Q, e, s}^{\circ}(P: \nu: m) \psi:=q_{\sigma_{s}, 0}(Q, e \mid f, 0)(\nu, m)$. Then (17.4) applied to $\psi$ follows for $\nu \in \Omega_{P}^{\prime \prime}$. Finally, the assertions on meromorphy follow from the fact that $q_{\sigma_{s}, 0}(Q, e \mid f, 0) \in \mathcal{M}\left(\mathfrak{a}_{P \mathrm{qC}}^{*}, \mathcal{H}_{f}, d_{f}, C^{\infty}\left(\mathrm{X}_{Q, e}: \tau_{Q}\right)\right)$, by Lemma 13.17

It remains to establish (b)-(d) under the assumption that $u \in N_{K}\left(\mathfrak{a}_{\mathrm{q}}\right)$ is arbitrary. Assertion (b) follows from the already established assertion with $u=e$ by application of Lemma 17.3 with $u, e$ in place of $u, v$, respectively.

To prove (c), we assume that $E_{Q, u}^{\circ}(P: \cdot) \neq 0$ and put $Q^{\prime}=u^{-1} Q u$. Using Lemma 17.3 we infer that $E_{Q^{\prime}, e}^{\circ}(P: \cdot) \neq 0$. Hence, from the already established assertion (c) with $Q^{\prime}, e$ in place of $Q, u$ it follows that $W\left(\mathfrak{a}_{P \mathrm{q}} \mid \mathfrak{a}_{Q^{\prime} \mathrm{q}}\right) \neq \emptyset$. Since $s \mapsto \operatorname{Ad}(u)^{-1} \circ s \circ \operatorname{Ad}(u)$ induces a bijection from $W\left(\mathfrak{a}_{P \mathrm{q}} \mid \mathfrak{a}_{Q \mathrm{q}}\right)$ onto $W\left(\mathfrak{a}_{P \mathrm{q}} \mid \mathfrak{a}_{Q^{\prime} \mathrm{q}}\right)$, it follows that $W\left(\mathfrak{a}_{P \mathrm{q}} \mid \mathfrak{a}_{Q \mathrm{q}}\right) \neq \emptyset$.

Finally, assertion (d) follows from the already established assertion with $u^{-1} Q u, e$ in place of $Q, u$ by applying Lemma 17.3 once more in a similar fashion as above.

If $P, Q$ are associated parabolic subgroups in $\mathcal{P}_{\sigma}$, see Def. 13.4 then $W\left(\mathfrak{a}_{P \mathrm{q}}\right.$ $\left.\mathfrak{a}_{Q \mathrm{q}}\right)$ is a non-empty finite set of isomorphisms from $\mathfrak{a}_{Q \mathrm{q}}$ onto $\mathfrak{a}_{P \mathrm{q}}$; moreover, the natural left action of $W\left(\mathfrak{a}_{P \mathrm{q}}\right)$ as well as the natural right action of $W\left(\mathfrak{a}_{Q \mathrm{q}}\right)$ on this set is free and transitive.

Proposition 17.5 (RT) Let $P, Q \in \mathcal{P}_{\sigma}$ be associated and let $v \in N_{K}\left(\mathfrak{a}_{\mathrm{q}}\right)$.

Then, for each $s \in W\left(\mathfrak{a}_{P \mathrm{q}} \mid \mathfrak{a}_{Q \mathrm{q}}\right)$ and every $\psi \in \mathcal{A}_{2, P}$, the meromorphic $C^{\infty}\left(\mathrm{X}_{Q, v}: \tau\right)$-valued function $\nu \mapsto E_{Q, v, s}^{\circ}(P: \nu: \cdot) \psi$ on $\mathfrak{a}_{P \mathrm{qc}}^{*}$, defined as in 17.4, attains its values in the finite dimensional space $\mathcal{A}_{2}\left(\mathrm{X}_{Q, v}: \tau_{Q}\right)$.

Proof: We give the proof under the assumption that $P$ and $Q$ are of residue type, see Remark 12.2 Fix $\psi \in \mathcal{A}_{2, P}$. Let $\nu \in \Omega_{P}$ and define the function $f \in \mathcal{A}_{\text {temp }}\left(\mathrm{X}_{1 Q, v}: \tau_{Q}\right)$ by

$$
f(m)=E_{Q, v}^{\circ}(P: \nu: m) \psi \quad\left(m \in \mathrm{X}_{Q, v}\right) .
$$

We recall from Section 2 that ${ }^{*} \mathfrak{a}_{Q \mathrm{q}}$ is maximal abelian in $\operatorname{Ad}(v) \mathfrak{q}$. Let $R_{Q}$ be a proper parabolic subgroup of $M_{Q}$ that contains ${ }^{*} A_{Q q}$ and is stable under the involution $\sigma^{v} \theta$. In the notation of Section $2 R_{Q}$ is of the form $P_{X}$, for some $X \in{ }^{*} \mathfrak{a}_{Q \mathrm{q}}$, relative to $\left(M_{Q}, \theta\right)$ in place of $(G, \theta)$. Since $X$ is fixed under $\sigma \theta$, it follows that $R_{Q}$ is $\sigma \theta$-stable as well. The $\sigma^{v}$-split component of the Lie algebra of $R_{Q}$ equals $\mathfrak{a}_{R_{Q}} \cap \operatorname{Ad}(v) \mathfrak{q}=\mathfrak{a}_{R_{Q}} \cap{ }^{*} \mathfrak{a}_{Q \mathfrak{q}}=\mathfrak{a}_{R_{Q}} \cap \mathfrak{q}$, hence equals the $\sigma$-split 
component. We therefore denote it by $\mathfrak{a}_{R_{Q} q}$; the associated positive chamber is denoted by $\mathfrak{a}_{R_{Q} \mathrm{q}}^{+}$.

Since $A_{Q}$ is central in $M_{1 Q}$, and stable under both $\sigma^{v}$ and $\sigma$, the group $R_{1 Q}=$ $R_{Q} A_{Q}$ is a parabolic subgroup of $M_{1 Q}$ that contains $A_{\mathrm{q}}$ and is stable under both involutions $\sigma^{v} \theta$ and $\sigma \theta$. The associated $\sigma^{v}$-split component equals $A_{\mathrm{q}}=$ ${ }^{*} A_{Q \mathrm{q}} A_{Q \mathrm{q}}$, which is also equal to the $\sigma$-split component of $R_{1 Q}$. Accordingly, the positive chamber is given by $A_{R_{1 Q \mathrm{q}}}^{+}=A_{R_{Q \mathrm{q}}}^{+} A_{Q \mathrm{q}}$.

We now claim that every $\xi \in \operatorname{Exp}\left(R_{1 Q}, e \mid f\right)$ satisfies

$$
\operatorname{Re} \xi+\rho_{R_{1 Q}} \leq 0 \quad \text { on } \quad \mathfrak{a}_{R_{1 Q \mathrm{q}}}^{+} \text {. }
$$

Indeed, for $R_{1 Q}$ minimal this follows from Lemma 10.2 For general $R_{1 Q}$, it follows by application of [14, Thm. 3.5.

On the other hand, it is readily seen that $R=R_{1 Q} N_{Q}$ is an element of $\mathcal{P}_{\sigma}$ and that $R_{1 Q}={ }^{* 1} R:=R \cap M_{1 Q}$. By application of Lemma 17.2

$$
f_{R_{1 Q}, e}=E_{R, v}^{\circ}(P: \nu) \psi \text {. }
$$

From $R_{Q} \subsetneq M_{Q}$ we infer that $R \subsetneq Q$, hence $\mathfrak{a}_{Q \mathrm{q}} \subsetneq \mathfrak{a}_{R \mathrm{q}}$, from which we see that $\operatorname{dim} \mathfrak{a}_{R \mathrm{q}}>\operatorname{dim} \mathfrak{a}_{P \mathrm{q}}$; hence, $W\left(\mathfrak{a}_{P \mathrm{q}} \mid \mathfrak{a}_{R \mathrm{q}}\right)=\emptyset$. From Proposition 17.4 (b) it now follows that the function on the right-hand side of the above equality is zero. We conclude that $f_{R_{1 Q}, e}=0$ for every $R_{Q}$ as above. By definition of the constant term it follows that every $\xi \in \operatorname{Exp}\left(R_{1 Q}, e \mid f\right)$ satisfies $\operatorname{Re} \xi+\rho_{R_{1 Q}} \neq 0$ in addition to (17.8). Put $f_{s}=E_{Q, v, s}^{\circ}(P: \nu) \psi$. Then

$$
f(a m)=\sum_{s \in W\left(\mathfrak{a}_{P \mathrm{q}} \mid \mathfrak{a}_{Q \mathrm{q}}\right)} a^{s^{*} \nu} f_{s}(m),
$$

for $m \in \mathrm{X}_{Q, v}$ and $a \in A_{Q \mathrm{q}}$. It follows that every $f_{s}$ belongs to $\mathcal{A}_{\text {temp }}\left(\mathrm{X}_{Q, v}: \tau_{Q}\right)$. Moreover, every $\xi \in \operatorname{Exp}\left(R_{Q}, e \mid f_{s}\right)$ satisfies $\operatorname{Re} \xi+\rho_{R_{Q}} \leq 0$ on $\mathfrak{a}_{R_{Q q}}^{+}$and $\operatorname{Re} \xi+\rho_{R_{Q}} \neq 0$. In particular, if $R_{Q}$ is a maximal $\theta \sigma^{v}$-stable parabolic subgroup in $M_{Q}$, it follows that every exponent $\xi \in \operatorname{Exp}\left(R_{Q}, e \mid f_{s}\right)$ satisfies $\operatorname{Re} \xi+\rho_{R_{Q}}<$ 0 on $\mathfrak{a}_{R_{Q} \mathrm{q}}^{+}$. This implies that $\left(f_{s}\right)_{R_{Q}, e}=0$; hence, $f_{s} \in \mathcal{A}_{2}\left(\mathrm{X}_{Q, v}: \tau_{Q}\right)$, by [9], Prop. 12 .

Let $s \in W\left(\mathfrak{a}_{P \mathrm{q}} \mid \mathfrak{a}_{Q \mathrm{q}}\right)$. We have shown that the function $\varphi: \nu \mapsto E_{Q, v, s}^{\circ}(P: \nu) \psi$ attains its values in $\mathcal{A}_{2}\left(\mathrm{X}_{Q, v}: \tau_{Q}\right)$ for $\nu \in \Omega_{P}$. Since $Q$ is of residue type, $\mathcal{A}_{2}\left(\mathrm{X}_{Q, v}: \tau_{Q}\right)$ is a finite dimensional subspace of $C^{\infty}\left(\mathrm{X}_{Q, v}: \tau_{Q}\right)$ by Lemma 12.6 By meromorphy it now follows that $\varphi$ is $\mathcal{A}_{2}\left(\mathrm{X}_{Q, v}: \tau_{Q}\right)$-valued.

If $P, Q \in \mathcal{P}_{\sigma}$ are associated, then $s \mapsto s^{-1}$ defines a bijection from $W\left(\mathfrak{a}_{Q q} \mid\right.$ $\left.\mathfrak{a}_{P \mathrm{q}}\right)$ onto $W\left(\mathfrak{a}_{P \mathrm{q}} \mid \mathfrak{a}_{Q \mathrm{q}}\right)$. In this case we write, for $s \in W\left(\mathfrak{a}_{Q \mathrm{q}} \mid \mathfrak{a}_{P \mathrm{q}}\right)$,

$$
s \nu:=\left(s^{-1}\right)^{*} \nu=\nu \circ s^{-1}, \quad\left(\nu \in \mathfrak{a}_{P \mathrm{qC}}^{*}\right) .
$$

Definition 17.6 (RT) Let $P, Q \in \mathcal{P}_{\sigma}$ be be associated. For each $s \in W\left(\mathfrak{a}_{Q q} \mid\right.$ $\left.\mathfrak{a}_{P \mathrm{q}}\right)$ we define the meromorphic $\operatorname{Hom}\left(\mathcal{A}_{2, P}, \mathcal{A}_{2, Q}\right)$-valued function $C_{Q \mid P}^{\circ}(s: \cdot)$ on $\mathfrak{a}_{P \mathrm{qc}}^{*}$ by

$$
\left[C_{Q \mid P}^{\circ}(s: \nu) \psi\right]_{v}=E_{Q, v, s^{-1}}^{\circ}(P: \nu) \psi, \quad\left(v \in{ }^{Q} \mathcal{W}\right)
$$


In the chain of reasoning leading up to Theorem 21.2 this definition requires $P$ to be of residue type, since it depends on the validity of Definition 13.7 see Remark 12.2

Corollary 17.7 (RT) Let $P, Q \in \mathcal{P}_{\sigma}$. For each $s \in W\left(\mathfrak{a}_{Q \mathrm{q}} \mid \mathfrak{a}_{P \mathrm{q}}\right)$, the $\operatorname{Hom}\left(\mathcal{A}_{2, P}, \mathcal{A}_{2, Q}\right)$ valued meromorphic function $C_{Q \mid P}^{\circ}(s: \cdot)$ on $\mathfrak{a}_{P \mathrm{qC}}^{*}$ has a singular locus equal to a locally finite union of real $\Sigma_{r}(P)$-hyperplanes.

Let $\nu \in i \mathfrak{a}_{P \mathrm{q}}^{*}$ be a regular point for $E^{\circ}(P: \cdot)$ and the $C$-functions $C_{Q \mid P}^{\circ}(s: \cdot)$, as $s \in W\left(\mathfrak{a}_{Q \mathrm{q}} \mid \mathfrak{a}_{P \mathrm{q}}\right)$. Let $\psi \in \mathcal{A}_{2, P}$. Then the function $E^{\circ}(P: \nu) \psi$, which belongs to $\mathcal{A}_{\text {temp }}(\mathrm{X}: \tau)$ by Proposition 13.15, has the following constant term along $(Q, v)$, for $v \in{ }^{Q} \mathcal{W}$,

$$
E_{Q, v}^{\circ}(P: \nu: m a) \psi=\sum_{s \in W\left(\mathfrak{a}_{Q \mathrm{q}} \mid \mathfrak{a}_{P q}\right)} a^{s \nu}\left[C_{Q \mid P}^{\circ}(s: \nu) \psi\right]_{v}(m),
$$

for all $m \in \mathrm{X}_{Q, v}$ and $a \in A_{Q \mathrm{q}}$.

Proof: We give the proof under the assumption that $P$ and $Q$ are of residue type, see Remark 12.2 The result is an immediate consequence of Propositions 17.4 and 17.5 combined with Definition 17.6

Remark 17.8 Formula (17.9) above generalizes Harish-Chandra's formula for the constant term of the normalized Eisenstein integral in 29, Thm. 5-6, see also 31, Thm. 14.1. Accordingly, the functions $C_{Q \mid P}^{\circ}(s: \cdot)$, for $s \in W\left(\mathfrak{a}_{Q \mathrm{q}} \mid \mathfrak{a}_{P \mathrm{q}}\right)$, will be called normalized $C$-functions.

In the context of reductive symmetric spaces, for minimal $P$ the above result is due to [5], Eqn. (133), in view of 9], Eqn. (52). For general $P$ the result is due to 19, Eqn. (5.3). See also Remark 13.10]

Remark 17.9 Note that it follows from the characterization of the normalized Eisenstein integral in Proposition [13.6 that

$$
C_{P \mid P}^{\circ}(1: \nu)=I, \quad\left(\nu \in \mathfrak{a}_{P \mathrm{qC}}^{*}\right) .
$$

\section{The Maass-Selberg relations}

In this section we derive the Maass-Selberg relations for the normalized $C$ functions. As a first step we use the vanishing theorem to prove the following functional equation for the Eisenstein integral.

Proposition 18.1 (RT) Let $P, Q \in \mathcal{P}_{\sigma}$ be associated parabolic subgroups. Then, for each $s \in W\left(\mathfrak{a}_{Q \mathrm{q}} \mid \mathfrak{a}_{P \mathrm{q}}\right)$ and all $x \in \mathrm{X}$,

$$
E^{\circ}(P: \nu: x)=E^{\circ}(Q: s \nu: x) C_{Q \mid P}^{\circ}(s: \nu),
$$

as a meromorphic identity in $\nu \in \mathfrak{a}_{P \mathrm{qC}}^{*}$. 
Proof: We give the proof under the assumption that $P$ is of residue type, see Remark 12.2 Fix $\psi \in \mathcal{A}_{2, P}$. By Corollary 17.7 the function $\nu \mapsto C_{Q \mid P}^{\circ}(s: \nu) \psi$ belongs to $\mathcal{M}\left(\mathfrak{a}_{P \mathrm{qC}}^{*}, \mathcal{H}, \mathcal{A}_{2, Q}\right)$, for some locally finite collection $\mathcal{H}$ of $\Sigma_{r}(P)$ hyperplanes in $\mathfrak{a}_{P \mathrm{qC}}^{*}$. It follows that the function $\lambda \mapsto C_{Q \mid P}^{\circ}\left(s: s^{-1} \lambda\right) \psi$ belongs to $\mathcal{M}\left(\mathfrak{a}_{Q \mathrm{qC}}^{*}, s \mathcal{H}, \mathcal{A}_{2, Q}\right)$. Here $s \mathcal{H}$ is a locally finite collection of $\Sigma_{r}(Q)$-hyperplanes in $\mathfrak{a}_{Q \mathrm{qC}}^{*}$. By Proposition 13.6 the family $(\lambda, x) \mapsto E^{\circ}(Q: \lambda: x) \psi_{Q}$ belongs to $\mathcal{E}_{Q}^{\text {hyp }}(\mathrm{X}: \tau)$, for every $\psi_{Q} \in \mathcal{A}_{2, Q}$. We conclude that the family

$$
f:(\lambda, x) \mapsto E^{\circ}(Q: \lambda: x) C_{Q \mid P}^{\circ}\left(s: s^{-1} \lambda\right) \psi
$$

belongs to $\mathcal{E}_{Q}^{\text {hyp }}(\mathrm{X}: \tau)$ as well. For $\lambda$ in the complement $\Omega$ of a locally finite union of hyperplanes in $i \mathfrak{a}_{Q \mathrm{q}}^{*}$, the function $f_{\lambda}$ belongs to $\mathcal{A}_{\text {temp }}(\mathrm{X}: \tau)$, and its constant term along $(Q, v)$, for $v \in{ }^{Q} \mathcal{W}$, is given by

$$
\left(f_{\lambda}\right)_{Q, v}(m a)=\sum_{t \in W\left(\mathfrak{a}_{Q q} \mid \mathfrak{a}_{Q q}\right)} a^{t \lambda}\left[\operatorname{pr}_{v} C_{Q \mid Q}^{\circ}(t: \lambda) C_{Q \mid P}^{\circ}\left(s: s^{-1} \lambda\right) \psi\right](m) ;
$$

see Corollary 17.7 Taking Remark [17.9 into account, we see that $q_{\lambda-\rho_{Q}}(Q, v \mid$ $f_{\lambda}(X, m)=\left[\operatorname{pr}_{v} C_{Q \mid P}^{\circ}\left(s: s^{-1} \lambda\right) \psi\right](m)$, for all $\lambda \in \Omega, X \in \mathfrak{a}_{Q \mathrm{q}}$ and $m \in \mathrm{X}_{Q, v,+}$. By application of [14], Thm. 7.7, Eqn. (7.14), it follows that

$$
q_{\overline{1}, 0}(Q, v \mid f, X)(\lambda, m)=\left[\operatorname{pr}_{v} C_{Q \mid P}^{\circ}\left(s: s^{-1} \lambda\right) \psi\right](m),
$$

for generic $\lambda \in i \mathfrak{a}_{Q \mathrm{q}}^{*}, X \in \mathfrak{a}_{Q \mathrm{q}}$ and $m \in \mathrm{X}_{Q, v,+}$. By meromorphy this actually holds as an identity of meromorphic functions in $\lambda$.

On the other hand, it follows from Definition 13.7 combined with Lemma 6.12 that the family

$$
g:(\lambda, x) \mapsto E^{\circ}\left(P: s^{-1} \lambda: x\right) \psi
$$

belongs to $\mathcal{E}_{Q}^{\text {hyp }}(\mathrm{X}: \tau)$. Moreover, for $\lambda$ in the complement of a locally finite union of hyperplanes in $i \mathfrak{a}_{Q \mathrm{q}}^{*}$, the function $g_{\lambda}$ belongs to $\mathcal{A}_{\text {temp }}(\mathrm{X}: \tau)$, and its constant term along $(Q, v)$ is given by

$$
\left(g_{\lambda}\right)_{Q, v}(m a)=\sum_{t \in W\left(\mathfrak{a}_{Q \mathbf{q}} \mid \mathfrak{a}_{P \mathrm{q}}\right)} a^{t s^{-1} \lambda}\left[\operatorname{pr}_{v} C_{Q \mid P}^{\circ}\left(t: s^{-1} \lambda\right) \psi\right](m) .
$$

This implies that, for every $X \in \mathfrak{a}_{Q \mathrm{q}}$,

$$
q_{\overline{1}, 0}(Q, v \mid g, X)(\lambda, \cdot)=\operatorname{pr}_{v} C_{Q \mid P}^{\circ}\left(s: s^{-1} \lambda\right) \psi,
$$

as a meromorphic identity in $\lambda \in \mathfrak{a}_{Q \mathrm{qC}}^{*}$. From (18.2) and (18.4) it follows that the family $f-g \in \mathcal{E}_{Q}^{\text {hyp }}(\mathrm{X}: \tau)$ satisfies the hypothesis of the vanishing theorem, Theorem 6.11 Hence, $f=g$. It follows that the meromorphic $C^{\infty}(\mathrm{X}: \tau)$-valued function $\nu \mapsto f_{s \nu}-g_{s \nu}$ on $\mathfrak{a}_{P \mathrm{qC}}^{*}$ is zero. This implies the result, in view of (18.1) and (18.3). 
Corollary 18.2 (RT) Let $P, Q \in \mathcal{P}_{\sigma}$ be associated parabolic subgroups. Then, for each $s \in W\left(\mathfrak{a}_{Q q} \mid \mathfrak{a}_{P q}\right)$ and all $x \in X$,

$$
E^{*}(P: \nu: x)=C_{Q \mid P}^{\circ}(s:-\bar{\nu})^{*} E^{*}(Q: s \nu: x),
$$

as a meromorphic identity in $\nu \in \mathfrak{a}_{Q \mathrm{qc}}^{*}$.

Proof: We give the proof under the assumption that $P$ and $Q$ are of residue type, see Remark 12.2 The result follows from Proposition 18.1 combined with Definition 13.7

We shall now derive the Maass-Selberg relations for the normalized $C$ functions from the invariance properties of the kernel $K_{P}$, formulated in Theorem 13.23

Theorem 18.3 (RT) Let $P, Q \in \mathcal{P}_{\sigma}$ be associated. Then for each $s \in W\left(\mathfrak{a}_{Q \mathrm{q}} \mid\right.$ $\left.\mathfrak{a}_{P \mathrm{q}}\right)$,

$$
C_{Q \mid P}^{\circ}(s: \nu) C_{Q \mid P}^{\circ}(s:-\bar{\nu})^{*}=I,
$$

as an identity of meromorphic $\operatorname{End}\left(\mathcal{A}_{2, Q}\right)$-valued functions in the variable $\nu \in$ $\mathfrak{a}_{P \mathrm{qC}}^{*} ;$

Remark 18.4 For the case of the group the above result was announced by Harish-Chandra in 29, Thm. 6, with a proof appearing in 32, Lemma 17.1 (see also Remark [13.9). For the Riemannian case $H=K$, which is a special case of that of the group, the relations were proved in [34, Thm. 6.6.

For general reductive symmetric spaces and minimal $P$ the result is due to [5], Thm. 16.3, combined with [6], in view of [9], text after Eqn. (55). For general $P$ the result is due to J. Carmona and P. Delorme, 19, Thm. 2 and Prop. 5 (vi). See also Remark 13.10

Proof: We give the proof under the assumption that $P$ and $Q$ are of residue type, see Remark 12.2 It follows from Definition 13.20 that

$$
\left|W_{Q}\right| K_{Q}(s \nu: x: y)=E^{\circ}(Q: s \nu: x) E^{*}(Q: s \nu: y),
$$

for all $x, y \in \mathrm{X}$, as an identity of meromorphic functions in $\nu \in \mathfrak{a}_{P \mathrm{qc}}^{*}$. On the other hand, from the mentioned definition combined with Proposition 18.1 and Corollary 18.2 it follows that

$$
\begin{aligned}
& \left|W_{P}\right| K_{P}(\nu: x: y)=E^{\circ}(P: \nu: x) E^{*}(P: \nu: y) \\
& \quad=E^{\circ}(Q: s \nu: x) C_{Q \mid P}^{\circ}(s: \nu) C_{Q \mid P}^{\circ}(s:-\bar{\nu})^{*} E^{*}(Q: s \nu: y),
\end{aligned}
$$

for $x, y \in \mathrm{X}$, and generic $\nu \in \mathfrak{a}_{P \mathrm{qC}}^{*}$. Now $\left|W_{P}\right|=\left|W_{Q}\right|$ since $P$ and $Q$ are associated. Hence, using Theorem 13.23 we infer that

$$
\begin{aligned}
& E^{\circ}(Q: s \nu: x) E^{*}(Q: s \nu: y) \\
& \quad=E^{\circ}(Q: s \nu: x) C_{Q \mid P}^{\circ}(s: \nu) C_{Q \mid P}^{\circ}(s:-\bar{\nu})^{*} E^{*}(Q: s \nu: y)
\end{aligned}
$$


for all $x, y \in X$, as identities of meromorphic functions in the variable $\nu \in \mathfrak{a}_{P \mathrm{qC}}^{*}$. Let $v \in{ }^{Q} \mathcal{W}$, and take $x=$ mav with $m \in \mathrm{X}_{Q, v,+}$ arbitrary and $a$ tending to infinity in $A_{Q \mathrm{q}}^{+}$. Comparing the coefficients of $a^{s \nu-\rho_{Q}}$ in the asymptotic expansions along $(Q, v)$ of the resulting expressions on both sides of (18.8), using Definition 13.7 we obtain that

$$
\operatorname{pr}_{Q, v} \circ E^{*}(Q: s \nu: y)=\operatorname{pr}_{Q, v} \circ C_{Q \mid P}^{\circ}(s: \nu) C_{Q \mid P}^{\circ}(s:-\bar{\nu})^{*} E^{*}(Q: s \nu: y),
$$

for all $y \in \mathrm{X}$, as an identity of meromorphic functions in the variable $\nu \in \mathfrak{a}_{P \mathrm{qc}}^{*}$. Taking adjoints and substituting $-\bar{\nu}$ for $\nu$ we now obtain

$$
E^{\circ}(Q: s \nu: y) \circ \mathrm{i}_{Q, v}=E^{\circ}(Q: s \nu: y) C_{Q \mid P}^{\circ}(s: \nu) C_{Q \mid P}^{\circ}(s:-\bar{\nu})^{*} \circ \mathrm{i}_{Q, v} .
$$

Fix $w \in{ }^{Q} \mathcal{W}$, and put $y=$ maw, with $m \in \mathrm{X}_{Q, w,+}$ arbitrary and $a \in A_{Q \mathrm{q}}^{+}$ tending to infinity. Comparing the coefficients of $a^{s \nu-\rho_{Q}}$ in the expansions along $(Q, w)$ of the functions on both sides of (18.9), we obtain

$$
\operatorname{pr}_{Q, w} \circ \mathrm{i}_{Q, v}=\operatorname{pr}_{Q, w} \circ C_{Q \mid P}^{\circ}(s: \nu) C_{Q \mid P}^{\circ}(s:-\bar{\nu})^{*} \circ \mathrm{i}_{Q, v},
$$

as a meromorphic identity in the variable $\nu \in \mathfrak{a}_{P \mathrm{qc}}^{*}$. This holds for arbitrary $v, w \in{ }^{Q} \mathcal{W}$; in view of the direct sum decomposition (13.1) with $Q$ in place of $P$, the equality (18.10) therefore remains valid if the maps $\operatorname{pr}_{Q, w}$ and $\mathrm{i}_{Q, v}$ are replaced by the identity map of $\mathcal{A}_{2, Q}$.

Remark 18.5 Conversely, if the Maass-Selberg relations (18.5) hold, then the expression on the right-hand side of (18.7) equals the one on the right-hand side of (18.6); hence (13.13), the invariance property of the kernel $K_{P}$, follows. Thus, the Maass-Selberg relations are equivalent to the invariance properties of the kernel.

Corollary 18.6 (RT) Let $P, Q \in \mathcal{P}_{\sigma}$ be associated parabolic subgroups. and let $s \in W\left(\mathfrak{a}_{Q \mathrm{q}} \mid \mathfrak{a}_{P \mathrm{q}}\right)$. Then there exists a constant $\varepsilon>0$ such that the meromorphic $\operatorname{Hom}\left(\mathcal{A}_{2, P}, \mathcal{A}_{2, Q}\right)$-valued function $C_{Q \mid P}^{\circ}(s: \cdot)$ is regular on $\mathfrak{a}_{P \mathrm{q}}^{*}(\varepsilon)$.

Proof: We give the proof under the assumption that $P$ and $Q$ are of residue type, see Remark 12.2 The corollary is a straightforward consequence of Corollary 17.7 and Theorem 18.3, combined with the lemma below.

Lemma 18.7 Let $P \in \mathcal{P}_{\sigma}$. Let $V$ be a complete locally convex space, and let $\varphi$ be a $V$-valued meromorphic function on $\mathfrak{a}_{P \mathrm{qC}}^{*}$, with singular locus $\operatorname{sing} \varphi$ contained in a locally finite collection of real $\Sigma_{r}(P)$-hyperplanes. Assume that for every $\lambda_{0} \in i \mathfrak{a}_{P \mathrm{q}}^{*}$ there exists an open neighborhood $\omega$ of $\lambda_{0}$ in $i \mathfrak{a}_{P \mathrm{q}}^{*}$ such that $\varphi$ is bounded on $\omega \backslash \operatorname{sing} \varphi$. Then there exists a $\varepsilon>0$ such that $\varphi$ is holomorphic on $\mathfrak{a}_{P \mathrm{q}}^{*}(\varepsilon)$. 
Proof: Since the collection $\mathcal{H}$ of singular hyperplanes of $\varphi$ is a real $\Sigma_{r}(P)$ configuration, the number of $H \in \mathcal{H}$ with $H \cap \mathfrak{a}_{P \mathrm{q}}^{*}\left(\varepsilon^{\prime}\right) \cap H \neq \emptyset$ is finite, for every $\varepsilon^{\prime}>0$. Hence, there exists a $p \in \Pi_{\Sigma_{r}(P), \mathbb{R}}\left(\mathfrak{a}_{P \mathrm{q}}^{*}\right)$ of minimal degree such that $\varphi_{p}: \lambda \mapsto p(\lambda) \varphi(\lambda)$ is holomorphic on a neighborhood of $i \mathfrak{a}_{P \mathrm{q}}^{*}$. Clearly $\varphi_{p}$ is holomorphic on $\mathfrak{a}_{P \mathrm{q}}^{*}(\varepsilon)$, for a suitable $\varepsilon>0$. Assume that $\operatorname{deg} p \geq 1$. Then there exists a $\alpha \in \Sigma_{r}(P)$ and a constant $c \in \mathbb{R}$ such that $l: \lambda \mapsto\langle\alpha, \cdot\rangle-c$ is a divisor of $p$. By minimality of $p$ it follows that $h:=i \mathfrak{a}_{P q}^{*} \cap l^{-1}(0)$ is non-empty. From the hypothesis we infer that $\varphi_{p}=0$ on $h$. By analytic continuation it follows that $\varphi_{p}=0$ on $h_{\mathbb{C}} \cap \mathfrak{a}_{P \mathrm{q}}^{*}(\varepsilon)=l^{-1}(0) \cap \mathfrak{a}_{P \mathrm{q}}^{*}(\varepsilon)$. By a straightforward argument involving power series expansion in the coordinate function $l$, it now follows that $l^{-1} \varphi_{p}$ is holomorphic on $\mathfrak{a}_{P \mathrm{q}}^{*}(\varepsilon)$. This contradicts the minimality of $p$. Hence, $\operatorname{deg} p=0$ and the result follows.

Theorem 18.8 (RT) Let $P \in \mathcal{P}_{\sigma}$. Then there exists a constant $\varepsilon>0$ such that $\nu \mapsto E^{\circ}(P: \nu)$ is a holomorphic $C^{\infty}\left(\mathrm{X}, \operatorname{Hom}\left(\mathcal{A}_{2, P}, V_{\tau}\right)\right)$-valued function on $\mathfrak{a}_{P \mathrm{q}}^{*}(\varepsilon)$.

Remark 18.9 For the group case the above result is due to Harish-Chandra 32. For general reductive symmetric spaces and for $P$ minimal, the result is due to [9], Thm. 2. For non-minimal $P$ it is due to [19], Thm. 3(i).

Proof: We give the proof under the assumption that $P$ is of residue type, see Remark 12.2. Let $\Lambda \in \mathrm{L}_{P}(\mathfrak{b}, \tau)$ and fix $\psi \in \mathcal{A}_{2, P}(\Lambda)$ and $\eta \in V_{\tau}^{*}$. Let $\varepsilon_{0}>0$; then the family $F:(\lambda, x) \mapsto \eta E^{\circ}(P: \lambda: x) \psi$ belongs to the space $\operatorname{II}_{\text {mer }}\left(\Lambda, \varepsilon_{0}\right)$ defined in 17, Def. 3. From Corollary [17.7] and Corollary [18.6] it follows that $F$ satisfies the hypotheses of [7, Thm. 2. Hence, there exists a $\varepsilon_{1}>0$ such that $F$ belongs to the space $\operatorname{II}^{\prime}\left(\Lambda, \varepsilon_{1}\right)$. In particular, this implies that $\lambda \mapsto F_{\lambda}$ is holomorphic on $\mathfrak{a}_{P \mathrm{q}}^{*}(\varepsilon)$ for some $\varepsilon>0$. The theorem now follows by linearity and finite dimensionality of $V_{\tau}$ and $\mathcal{A}_{2, P}$.

Proposition 18.10 (RT) Let $P \in \mathcal{P}_{\sigma}$. Then there exist constants $\varepsilon>0$ and $s>0$ and for every $u \in U(\mathfrak{g})$ constants $n \in \mathbb{N}$ and $C>0$, such that the function $\nu \mapsto E^{\circ}(P: \nu)$ is a holomorphic $C^{\infty}\left(\mathrm{X}, \operatorname{Hom}\left(\mathcal{A}_{2, P}, V_{\tau}\right)\right)$-valued function on $\mathfrak{a}_{P \mathrm{q}}^{*}(\varepsilon)$ satisfying the estimate

$$
\left\|E^{\circ}(P: \nu: u ; x)\right\| \leq C|(\nu, x)|^{n} \Theta(x) e^{s|\operatorname{Re} \nu| l_{\mathrm{X}}(x)}, \quad\left(\nu \in \mathfrak{a}_{P \mathrm{q}}^{*}(\varepsilon), x \in \mathrm{X}\right) .
$$

Proof: We give the proof under the assumption that $P$ is of residue type, see Remark 12.2 By finite dimensionality of $V_{\tau}$ and $\mathcal{A}_{2, P}$, it follows from Theorem 15.12 and Definition 15.4 that there exists a $p \in \Pi_{\Sigma_{r}(P), \mathbb{R}}\left(\mathfrak{a}_{P \mathrm{q}}^{*}\right)$ and constants $\varepsilon>0$ and $s>0$ such that $\nu \mapsto p(\nu) E^{\circ}(P: \nu)$ is a holomorphic function on $\mathfrak{a}_{P q}^{*}(\varepsilon)$, with values in $C^{\infty}(\mathrm{X}) \otimes \operatorname{Hom}\left(\mathcal{A}_{2, P}, V_{\tau}\right)$. Moreover, it satisfies the following estimates. For every $u \in U(\mathfrak{g})$ there exist constants $n \in \mathbb{N}$ and $C>0$ such that

$$
\left\|p(\nu) E^{\circ}(P: \nu: u ; x)\right\| \leq C|(\nu, x)|^{n} \Theta(x) e^{s|\operatorname{Re} \nu| l_{\mathrm{X}}(x)}, \quad\left(\nu \in \mathfrak{a}_{P \mathrm{q}}^{*}(\varepsilon), x \in \mathrm{X}\right) .
$$


If we choose $\varepsilon>0$ sufficiently small, then by Theorem 18.8 the function $\nu \mapsto$ $E^{\circ}(P: \nu)$ is already holomorphic on $\mathfrak{a}_{P \mathrm{q}}^{*}(\varepsilon)$. By a straightforward application of Cauchy's integral formula, involving polydiscs of size $O\left(\left(1+l_{\mathrm{X}}(x)\right)^{-1}\right)$, it follows that for $\varepsilon>0$ sufficiently small, the following is true. For every $u \in U(\mathfrak{g})$ there exist $n \in \mathbb{N}$ and $C>0$ such that the estimate (18.11) holds with $p=1$.

Corollary 18.11 (RT) Let $P \in \mathcal{P}_{\sigma}$. Then there exist constants $\varepsilon>0$ and $s>0$ and for every $u \in U(\mathfrak{g})$ constants $n \in \mathbb{N}$ and $C>0$, such that the function $\nu \mapsto$ $E^{*}(P: \nu)$ is a holomorphic $C^{\infty}\left(\mathrm{X}, \operatorname{Hom}\left(V_{\tau}, \mathcal{A}_{2, P}\right)\right)$-valued function on $\mathfrak{a}_{P \mathrm{q}}^{*}(\varepsilon)$ satisfying the estimate

$$
\left\|E^{*}(P: \nu: u ; x)\right\| \leq C|(\nu, x)|^{n} \Theta(x) e^{s|\operatorname{Re} \nu| l_{\mathrm{X}}(x)}, \quad\left(\nu \in \mathfrak{a}_{P \mathrm{q}}^{*}(\varepsilon), x \in \mathrm{X}\right) .
$$

Proof: We give the proof under the assumption that $P$ is of residue type, see Remark 12.2 In view of Definition 13.7 the result follows from Proposition 18.10

Corollary 18.12 (RT) Let $P \in \mathcal{P}_{\sigma}$. Then, for all $U \in S\left(\mathfrak{a}_{P \mathrm{q}}^{*}\right)$ and $u \in U(\mathfrak{g})$, there exist constants $m \in \mathbb{N}$ and $C>0$ such that

$$
\left\|E^{*}(P: \nu ; U: u ; x)\right\| \leq C|(\nu, x)|^{m} \Theta(x), \quad\left(\nu \in i \mathfrak{a}_{P \mathrm{q}}^{*}, x \in \mathrm{X}\right) .
$$

Proof: We give the proof under the assumption that $P$ is of residue type, see Remark 12.2. The result follows from the estimate of the previous corollary, by a straightforward application of Cauchy's integral formula involving polydiscs of size $O\left(\left(1+l_{\mathrm{X}}(x)\right)^{-1}\right)$.

\section{The spherical Fourier transform}

We recall from [5], Cor. 17.6, that there exists a constant $N \in \mathbb{N}$ such that

$$
\left(1+l_{\mathrm{X}}\right)^{-N} \Theta^{2} \in L^{1}(\mathrm{X})
$$

Combining the estimate (12.1) with (19.1) and the estimate of Corollary 18.12 we see that the integral in the following definition converges absolutely.

Definition 19.1 (RT) Let $P \in \mathcal{P}_{\sigma}$. If $f \in \mathcal{C}(\mathrm{X}: \tau)$, we define its Fourier transform $\mathcal{F}_{P} f: i \mathfrak{a}_{P \mathrm{q}}^{*} \rightarrow \mathcal{A}_{2, P}$ by

$$
\mathcal{F}_{P} f(\nu)=\int_{X} E^{*}(P: \nu: x) f(x) d x, \quad\left(\nu \in i \mathfrak{a}_{P \mathrm{q}}^{*}\right) .
$$

The above definition depends on the validity of the estimate of Corollary 18.12 Thus, within the chain of reasoning leading up to Theorem 21.2 the use of this definition requires $P$ to be of residue type, see Remark 12.2 
Remark 19.2 If $G$ has compact center modulo $H$, then $\mathfrak{a}_{G q}=\{0\}$ and $\mathcal{A}_{2, G}=$ $\mathcal{A}_{2}(G / H: \tau)$. Moreover, using Remark [13.11 we infer that $f \mapsto \mathcal{F}_{G} f(0)$ is the restriction to $\mathcal{C}(\mathrm{X}: \tau)$ of the orthogonal projection $L^{2}(\mathrm{X}: \tau) \rightarrow L_{d}^{2}(\mathrm{X}: \tau)=$ $\mathcal{A}_{2, G}$.

Lemma 19.3 (RT) Let $P \in \mathcal{P}_{\sigma}$. Then for every $U \in S\left(\mathfrak{a}_{P \mathrm{q}}^{*}\right)$ there exists a constant $m \in \mathbb{N}$ and a continuous seminorm $s$ on $\mathcal{C}(\mathrm{X}: \tau)$ such that

$$
\sup _{\nu \in i \mathfrak{a}_{P \mathfrak{q}}^{*}}(1+|\nu|)^{-m}\left\|\mathcal{F}_{P} f(\nu ; U)\right\| \leq s(f),
$$

for all $f \in \mathcal{C}(\mathrm{X}: \tau)$. In particular, the Fourier transform $\mathcal{F}_{P}$ maps $\mathcal{C}(\mathrm{X}: \tau)$ continuous linearly into $C^{\infty}\left(i \mathfrak{a}_{P \mathrm{q}}^{*}\right) \otimes \mathcal{A}_{2, P}$.

Proof: We give the proof under the assumption that $P$ is of residue type, see Remark 12.2. The result follows from the estimates (19.1) and (12.1) combined with the estimate of Corollary 18.12

Lemma $19.4(\mathrm{RT})$ Let $P \in \mathcal{P}_{\sigma}$. Then for every $D \in \mathbb{D}(\mathrm{X})$ and all $f \in$ $\mathcal{C}(\mathrm{X}: \tau)$

$$
\mathcal{F}_{P}(D f)(\nu)=\underline{\mu}_{P}(D: \nu) \mathcal{F}_{P} f(\nu), \quad\left(\nu \in i \mathfrak{a}_{P \mathrm{q}}^{*}\right) .
$$

Proof: We give the proof under the assumption that $P$ is of residue type, see Remark 12.2 From [3], Lemma 7.2, we recall that every $D \in \mathbb{D}(\mathrm{X})$ acts by a continuous linear endomorphism on $\mathcal{C}(\mathrm{X}: \tau)$. Since $\mathcal{F}_{P}: \mathcal{C}(X: \tau) \rightarrow C_{c}^{\infty}\left(i \mathfrak{a}_{P \mathrm{q}}^{*}\right) \otimes \mathcal{A}_{2, P}$ is continuous, it suffices to prove the identity (19.2) for $f$ in the dense subspace $C_{c}^{\infty}(\mathrm{X}: \tau)$ of $\mathcal{C}(\mathrm{X}: \tau)$. For such $f$ the identity is an immediate consequence of Lemma 14.8

Let $\Omega$ be the image in $\mathbb{D}(\mathrm{X})$ of the Casimir operator defined by the bilinear form $B$ on $\mathfrak{g}$, see Section 2

Lemma $19.5(\mathrm{RT})$ Let $P \in \mathcal{P}_{\sigma}$ and let $\varepsilon>0$.

(a) $\underline{\mu}_{P}(\Omega: \nu)=-|\nu|^{2} I+O(|\nu|)$ as $\nu \in \mathfrak{a}_{P \mathrm{q}}^{*}(\varepsilon),|\nu| \rightarrow \infty$.

(b) There exists a constant $R>0$ such that for every $\nu \in \mathfrak{a}_{P \mathrm{q}}^{*}(\varepsilon)$ with $|\nu| \geq R$ the endomorphism $\underline{\mu}_{P}(\Omega: \nu)$ is invertible and the operator norm of its inverse satisfies the estimate

$$
|\nu|^{2}\left\|\underline{\mu}_{P}(\Omega: \nu)^{-1}\right\| \leq 2 .
$$

Proof: We give the proof under the assumption that $P$ is of residue type, see Remark 12.2 Let $\langle\cdot, \cdot\rangle$ denote the complex bilinear form on $\mathfrak{a}_{P \mathrm{qC}}^{*}$ that extends the dual of the given bilinear form on $\mathfrak{a}_{P \mathrm{q}}$. There exists a first order polynomial function $\underline{\mu}_{1}: \mathfrak{a}_{P \mathrm{qC}}^{*} \rightarrow \operatorname{End}\left(\mathcal{A}_{2, P}\right)$ such that

$$
\underline{\mu}_{P}(\Omega: \nu)=\langle\nu, \nu\rangle I+\underline{\mu}_{1}(\nu), \quad\left(\nu \in \mathfrak{a}_{P \mathrm{qC}}^{*}\right) .
$$


Indeed, this follows by application of Corollary 14.4. It follows by a straightforward estimation that, for $\nu \in \mathfrak{a}_{P \mathrm{q}}^{*}(\varepsilon)$,

$$
\langle\nu, \nu\rangle=-|\nu|^{2}+O(|\nu|) \quad \text { as } \quad|\nu| \rightarrow \infty .
$$

Using that $\left\|\mu_{1}(\nu)\right\|=O(|\nu|)$ we obtain (a) from (19.4) and (19.5). From (a) it follows that $-|\nu|^{-2} \underline{\mu}_{P}(\Omega: \nu)=I+O\left(|\nu|^{-1}\right)$. Hence, (b) follows.

In the following result, $\mathcal{S}\left(i \mathfrak{a}_{P \mathrm{q}}^{*}\right)$ denotes the Euclidean Schwartz space of $i \mathfrak{a}_{P \mathrm{q}}^{*}$.

Proposition 19.6 (RT) Let $P \in \mathcal{P}_{\sigma}$. Then the Fourier transform $\mathcal{F}_{P}$ maps $\mathcal{C}(\mathrm{X}: \tau)$ continuous linearly into $\mathcal{S}\left(i \mathfrak{a}_{P \mathrm{q}}^{*}\right) \otimes \mathcal{A}_{2, P}$.

Proof: We give the proof under the assumption that $P$ is of residue type, see Remark 12.2 Moreover, we use the argumentation of [5], p. 436, completion of the proof of Theorem 19.1, with $\mathcal{F}_{P}$ in place of $\mathcal{F}_{\pi}$. Let us label the first two displayed formulas in the mentioned text in [5] by (E1) and (E2), respectively. The estimate (19.3) generalizes the estimate of [5], Lemma 19.4.

Let $R>0$ be as in Lemma 19.5 (b) for some choice of $\varepsilon>0$ and let $u \in S\left(\mathfrak{a}_{P \mathrm{q}}^{*}\right)$ and $M \in \mathbb{N}$. In view of the last assertion of Lemma 19.3 it suffices to prove the analogue of (E1), i.e., it suffices to prove the existence of a continuous seminorm $s$ on $\mathcal{C}(\mathrm{X}: \tau)$ such that

$$
\left\|\mathcal{F}_{P} f(\nu ; u)\right\| \leq(1+|\nu|)^{-M} s(f),
$$

for every $f \in \mathcal{C}(\mathrm{X}: \tau)$ and all $\nu \in i \mathfrak{a}_{P \mathrm{q}}^{*}$ with $|\nu| \geq R$. As in [5] this is done by induction on the order of $u$, by using Lemma 19.3 instead of (E2) and by using Lemmas 19.4 and 19.5 instead of [5], Lemmas 19.3 and 19.4, respectively.

We end this section with a result on the Fourier transform of a compactly supported smooth function. If $S>0$ we write

$$
\mathrm{X}_{S}:=\left\{x \in \mathrm{X} \mid l_{\mathrm{X}}(x) \leq S\right\} .
$$

Then $\mathrm{X}_{S}$ is a $K$-invariant compact subset of $\mathrm{X}$. We write $C_{S}^{\infty}(\mathrm{X}: \tau)$ for the closed subspace of $C^{\infty}(\mathrm{X}: \tau)$ consisting of functions with support contained in $\mathrm{X}_{S}$.

Proposition 19.7 (RT) Let $P \in \mathcal{P}_{\sigma}$ and let $\varepsilon>0$ be as in Corollary 18.11. For every $f \in C_{c}^{\infty}(\mathrm{X}: \tau)$, the Fourier transform $\mathcal{F}_{P}(f)$ extends to a holomorphic function on $\mathfrak{a}_{P \mathrm{q}}^{*}(\varepsilon)$ with values in $\mathcal{A}_{2, P}$. Moreover, let $S>0$. Then for every $m \in \mathbb{N}$ there exists a continuous seminorm $p_{m}$ on $C_{S}^{\infty}(\mathrm{X}: \tau)$ such that, for every $f \in C_{S}^{\infty}(\mathrm{X}: \tau)$,

$$
\left\|\mathcal{F}_{P} f(\nu)\right\| \leq(1+|\nu|)^{-m} p_{m}(f), \quad\left(\nu \in \mathfrak{a}_{P \mathrm{q}}^{*}(\varepsilon)\right) .
$$


Proof: We give the proof under the assumption that $P$ is of residue type, see Remark 12.2 The assertion about holomorphy is a straightforward consequence of the holomorphy of the Eisenstein integral as formulated in Corollary 18.11] Let $n \in \mathbb{N}$ be the constant of the mentioned corollary associated with $u=1$. Let $S>0$. Then it straightforwardly follows from the estimate of Corollary 18.11 that there exists a continuous seminorm $p_{0}$ on $C_{S}^{\infty}(\mathrm{X}: \tau)$ such that, for every $f \in C_{S}^{\infty}(\mathrm{X}: \tau)$,

$$
\left\|\mathcal{F}_{P} f(\nu)\right\| \leq(1+|\nu|)^{n} p_{0}(f), \quad\left(\nu \in \mathfrak{a}_{P q}^{*}(\varepsilon)\right) .
$$

Let $R>0$ be associated with $\varepsilon>0$ as in Lemma 19.5 Then it follows from the above estimate that, for every $k \in \mathbb{N}$ and for $\nu \in \mathfrak{a}_{P \mathrm{q}}^{*}(\varepsilon)$ with $|\nu| \geq R$,

$$
\begin{aligned}
|\nu|^{2 k}\left\|\mathcal{F}_{P} f(\nu)\right\| & =|\nu|^{2 k}\left\|\underline{\mu}_{P}(\Omega: \nu)^{-k} \mathcal{F}_{P}\left(\Omega^{k} f\right)(\nu)\right\| \\
& \leq(1+|\nu|)^{n} p_{0}\left(2^{k} \Omega^{k} f\right) .
\end{aligned}
$$

Taking $k \in \mathbb{N}$ such that $n-2 k \leq m$ we see that there exists a continuous seminorm $p_{m}^{\prime}$ on $C_{S}(\mathrm{X}: \tau)$ such that for every $f \in C_{S}^{\infty}(\mathrm{X}: \tau)$ the estimate (19.6) holds for all $\nu \in \mathfrak{a}_{P \mathrm{q}}^{*}(\varepsilon)$ with $|\nu| \geq R$. From (19.7) it follows that there exists a constant $C>0$ such that the estimate (19.6) holds with $C p_{0}$ in place of $p_{m}$, for all $\nu \in \mathfrak{a}_{P \mathrm{q}}^{*}(\varepsilon)$ with $|\nu|<R$. Take for $p_{m}$ any continuous seminorm with $p_{m} \geq \max \left(C p_{0}, p_{m}^{\prime}\right)$; then the desired assertion follows.

We end this section with another useful result.

Lemma $19.8(\mathrm{RT})$ Let $P \in \mathcal{P}_{\sigma}$ and assume that $\mathfrak{a}_{P \mathrm{q}} \neq 0$. Then $\mathcal{F}_{P}$ vanishes on $\mathcal{A}_{2}(\mathrm{X}: \tau)$.

Proof: We give the proof under the assumption that $P$ is of residue type, see Remark 12.2 Fix $f \in \mathcal{A}_{2}(\mathrm{X}: \tau)$. Then there exists a non-trivial polynomial $q$ in one variable such that $q(\Omega) f=0$. In view of Lemma 19.4 it follows that $q\left(\underline{\mu}_{P}(\Omega: \nu)\right) \mathcal{F}_{P} f(\nu)=0$ for all $\nu \in i \mathfrak{a}_{P \mathrm{q}}^{*}$. From Lemma 19.5 it follows that the polynomial function $\nu \mapsto \operatorname{det} q\left(\underline{\mu}_{P}(\Omega: \nu)\right)$ is not identically zero. Hence, $\mathcal{F}_{P} f$ vanishes on an open dense subset of $i \mathfrak{a}_{P q}^{*}$. By smoothness of $\mathcal{F}_{P} f$ it follows that $\mathcal{F}_{P} f=0$.

\section{The wave packet transform}

It follows from the estimate in Proposition 18.10 that the integral in the following definition is absolutely convergent. We agree to write $d \nu$ for the Lebesgue measure $d \mu_{P}(\nu)$ on $i \mathfrak{a}_{P \mathrm{q}}^{*}$, normalized as in Section 5

Definition 20.1 (RT) Let $P \in \mathcal{P}_{\sigma}$. Then for every $\varphi \in \mathcal{S}\left(i \mathfrak{a}_{P \mathrm{qC}}^{*}\right) \otimes \mathcal{A}_{2, P}$, we define the wave packet $\mathcal{J}_{P} \varphi: \mathrm{X} \rightarrow V_{\tau}$ by

$$
\mathcal{J}_{P} \varphi(x):=\int_{i \mathfrak{a}_{P \mathrm{q}}^{*}} E^{\circ}(P: \nu: x) \varphi(\nu) d \nu \quad(x \in \mathrm{X}) .
$$


This definition depends on the validity of the estimate of Proposition 18.10, which in the chain of reasoning leading up to Theorem 21.2 requires $P$ to be of residue type, see Remark 12.2

Note that the wave packet $\mathcal{J}_{P} \varphi$ is smooth $\tau$-spherical function.

Remark 20.2 If $G$ has compact center modulo $H$, then $A_{G q}=\{0\}$ and $\mathcal{A}_{2, G}=$ $\mathcal{A}_{2}(G / H: \tau)$. In this case the measure $d \nu=d \mu_{G}$ has total volume 1 (end of Section 5), and using Remark 13.11 we infer that $\mathcal{J}_{G} \varphi=\varphi(0)$. Accordingly, $\mathcal{J}_{G}$ is naturally identified with the inclusion map $\mathcal{A}_{2}(\mathrm{X}: \tau) \rightarrow C^{\infty}(\mathrm{X}: \tau)$.

Theorem 20.3 (RT) Let $P \in \mathcal{P}_{\sigma}$ Then the wave packet map $\mathcal{J}_{P}$ maps $\mathcal{S}\left(i \mathfrak{a}_{P \mathrm{q}}^{*}\right) \otimes$ $\mathcal{A}_{2, P}$ continuous linearly into the $S$ chwartz space $\mathcal{C}(\mathrm{X}: \tau)$.

Proof: We give the proof under the assumption that $P$ is of residue type, see Remark 12.2 Let $\Lambda \in \mathrm{L}_{P}(\mathfrak{b}, \tau)$ and fix $\psi \in \mathcal{A}_{2, P}(\Lambda)$. We recall from the proof of Theorem 18.8 that the family $F$ defined by

$$
F(\nu, x)=E^{\circ}(P: \nu: x) \psi
$$

has components with respect to a basis of $V_{\tau}$ that are functions of type $\operatorname{II}^{\prime}(\Lambda)$ in the sense of [7. Hence, by [7, Thm. 1, the map $\alpha \mapsto \mathcal{W}_{\alpha, F}$, where

$$
\mathcal{W}_{\alpha, F}(x)=\int_{i \mathfrak{a}_{P \mathrm{q}}^{*}} \alpha(\nu) F(\nu, x) d \nu,
$$

is continuous linear from $\mathcal{S}\left(i \mathfrak{a}_{P \mathrm{q}}^{*}\right)$ into $\mathcal{C}(\mathrm{X}: \tau)$. We note that $\mathcal{W}_{\alpha, F}=\mathcal{J}_{P}(\alpha \otimes \psi)$. Hence, the result follows by using linearity, the finite dimensionality of $\mathcal{A}_{2, P}$ (see Corollary 14.4) and the decomposition (14.10) of the latter space.

Let $P \in \mathcal{P}_{\sigma}$ and $D \in \mathbb{D}(\mathrm{X})$. In the following lemma we write $\underline{\mu}_{P}(D)$ for the endomorphism of $\mathcal{S}\left(i \mathfrak{a}_{P q}^{*}\right) \otimes \mathcal{A}_{2, P}$ given by

$$
\left[\underline{\mu}_{P}(D) \varphi\right](\nu)=\underline{\mu}_{P}(D: \nu)(\varphi(\nu)),
$$

for $\varphi \in \mathcal{S}\left(i \mathfrak{a}_{P \mathrm{q}}^{*}\right) \otimes \mathcal{A}_{2, P}$ and $\nu \in i \mathfrak{a}_{P \mathrm{q}}^{*}$

Lemma 20.4 (RT) Let $P \in \mathcal{P}_{\sigma}$ and $D \in \mathbb{D}(\mathrm{X})$. Then

$$
D \circ \mathcal{J}_{P}=\mathcal{J}_{P} \circ \underline{\mu}_{P}(D) \quad \text { on } \quad \mathcal{S}\left(i \mathfrak{a}_{P \mathrm{q}}^{*}\right) \otimes \mathcal{A}_{2, P}
$$

Proof: We give the proof under the assumption that $P$ is of residue type, see Remark 12.2 The operator $D$ defines a continuous linear endomorphism of $\mathcal{C}(\mathrm{X}: \tau)$, by 3 , Lemma 7.2. In view of Theorem 20.3 it follows that both sides of the equation are continuous linear maps from $\mathcal{S}\left(i \mathfrak{a}_{P q}^{*}\right) \otimes \mathcal{A}_{2, P}$ to $\mathcal{C}(\mathrm{X}: \tau)$. Hence, by density it suffices to prove the equality when applied to an element $\varphi \in C_{c}^{\infty}\left(i \mathfrak{a}_{P q}^{*}\right) \otimes \mathcal{A}_{2, P}$. But then the result is an immediate consequence of Lemma 14.1 by differentiation under the integral sign, in view of Definition 20.1 
We equip $\mathcal{C}(\mathrm{X}: \tau)$ with the restriction of the $L^{2}$-inner product $\langle\cdot \mid \cdot\rangle$ from $L^{2}(\mathrm{X}: \tau)$. Similarly, for $P \in \mathcal{P}_{\sigma}$, we equip $\mathcal{S}\left(i \mathfrak{a}_{P \mathrm{q}}^{*}\right) \otimes \mathcal{A}_{2, P}$ with the restriction of the $L^{2}$-type inner product $\langle\cdot \mid \cdot\rangle$ from $L^{2}\left(i \mathfrak{a}_{P \mathrm{q}}^{*}\right) \otimes \mathcal{A}_{2, P}$. With respect to these structures the Fourier transform $\mathcal{F}_{P}$ and the wave packet map $\mathcal{J}_{P}$ are adjoint in the following sense.

Lemma 20.5 (RT) Let $P \in \mathcal{P}_{\sigma}$. Then the continuous linear operators $\mathcal{F}_{P}: \mathcal{C}(\mathrm{X}: \tau) \rightarrow$ $\mathcal{S}\left(i \mathfrak{a}_{P \mathrm{q}}^{*}\right) \otimes \mathcal{A}_{2, P}$ and $\mathcal{J}_{P}: \mathcal{S}\left(i \mathfrak{a}_{P \mathrm{q}}^{*}\right) \otimes \mathcal{A}_{2, P} \rightarrow \mathcal{C}(\mathrm{X}: \tau)$ are adjoint in the sense that, for all $f \in \mathcal{C}(\mathrm{X}: \tau)$ and $\varphi \in \mathcal{S}\left(i \mathfrak{a}_{P \mathrm{q}}^{*}\right) \otimes \mathcal{A}_{2, P}$,

$$
\left\langle\mathcal{F}_{P} f \mid \varphi\right\rangle=\left\langle f \mid \mathcal{J}_{P} \varphi\right\rangle
$$

Proof: We give the proof under the assumption that $P$ is of residue type, see Remark 12.2. By continuity and density it suffices to prove (20.2) for all $f \in C_{c}^{\infty}(\mathrm{X}: \tau)$ and $\varphi \in C_{c}^{\infty}\left(i \mathfrak{a}_{P \mathrm{q}}^{*}\right) \otimes \mathcal{A}_{2, P}$. For such $f$ and $\varphi$, the formula follows by an application of Fubini's theorem.

\section{Fourier inversion for Schwartz functions}

In this section we show that the Fourier inversion formula [8.10), established in [12, implies an inversion formula for Schwartz functions, formulated in terms of the Fourier transforms and the wave packet maps introduced in the previous sections.

The crucial first step is the following.

Proposition 21.1 (RT) Let $F \subset \Delta$. Then for every $W$-invariant even residue weight $t \in \mathrm{WT}(\Sigma)$,

$$
T_{F}^{t}=\left[W: W_{F}\right] t\left(\mathfrak{a}_{F \mathrm{q}}^{+}\right) \mathcal{J}_{F} \mathcal{F}_{F} \quad \text { on } \quad C_{c}^{\infty}(\mathrm{X}: \tau) .
$$

Proof: We give the proof under the assumption that $P_{F}$ is of residue type, see Remark 12.2 In case $F=\Delta$ and $G$ has compact center modulo $H$, then $\mathfrak{a}_{F q}=$ $\mathfrak{a}_{G q}=\{0\}$ and the proof below has to be read according to the conventions indicated in Remarks 8.8 9.4 13.11, 19.2 and 20.2

Let $f \in C_{c}^{\infty}(\mathrm{X}: \tau)$ and $x \in \mathrm{X}_{+}$. It follows from (8.8) that

$$
T_{F}^{t} f(x)=|W| t\left(\mathfrak{a}_{F \mathrm{q}}^{+}\right) \int_{i \mathfrak{a}_{F \mathfrak{q}}^{*}+\varepsilon_{F}} \int_{X} K_{F}^{t}(\nu: x: y) f(y) d y d \mu_{F}(\nu),
$$

for all $\varepsilon_{F} \in \mathfrak{a}_{F \mathrm{q}}^{+}$sufficiently close to zero. In view of (13.12) and Definition 19.1 this equality may be rewritten as

$$
T_{F}^{t} f(x)=\left[W: W_{F}\right] t\left(\mathfrak{a}_{F \mathrm{q}}^{+}\right) \int_{i \mathfrak{a}_{F \mathfrak{q}}^{*}+\varepsilon_{F}} E^{\circ}\left(P_{F}: \nu: x\right) \mathcal{F}_{F} f(\nu) d \mu_{F}(\nu) .
$$

Since the expressions on both sides of the equation extend smoothly to all of $\mathrm{X}$ in the variable $x$, it follows that (21.2) holds for all $x \in \mathrm{X}$. From Theorem[18.8 
and Proposition 19.7 it follows that $\nu \mapsto E^{\circ}\left(P_{F}: \nu: x\right) \mathcal{F}_{F} f(\nu)$ is holomorphic on $\mathfrak{a}_{F \mathbf{q}}^{*}(\varepsilon)$, for some $\varepsilon>0$. Moreover, from the mentioned results it also follows that for every $N>0$, there exists a constant $C_{N}>0$ such that

$$
\left\|E^{\circ}\left(P_{F}: \nu: x\right) \mathcal{F}_{F} f(\nu)\right\| \leq C_{N}(1+|\nu|)^{-N}, \quad\left(\nu \in \mathfrak{a}_{F \mathrm{q}}^{*}(\varepsilon)\right) .
$$

This estimate allows us to take the limit of 21.2 for $\varepsilon_{F} \rightarrow 0$; thus, using Definition 20.1 and observing that $d \mu_{F}(\nu)=d \nu$ on $i \mathfrak{a}_{F \mathrm{q}}^{*}$, we obtain (21.1).

The proof of the following result involves an induction step using the long chain of results marked (RT), see Remark 12.2

\section{Theorem 21.2}

(a) Every $P \in \mathcal{P}_{\sigma}$ is of residue type.

(b) If $t$ is any $W$-invariant even residue weight for $\Sigma$, then

$$
f=\sum_{F \subset \Delta}\left[W: W_{F}\right] t\left(\mathfrak{a}_{F \mathrm{q}}^{+}\right) \mathcal{J}_{F} \mathcal{F}_{F} f
$$

for every $f \in \mathcal{C}(\mathrm{X}: \tau)$.

(c) The pair $(G, H)$ is of residue type if and only if $G$ has a compact center modulo $H$.

Proof: We first show that (c) and (b) follow from (a). Thus, assume (a). Then viewed as a parabolic subgroup, $G$ is of residue type. By Remark 13.3 it follows that the pair $\left({ }^{\circ} G,{ }^{\circ} G \cap H\right)$ is of residue type. Moreover, if $G$ has compact center modulo $H$, then $(G, H)$ is of residue type. If the center of $G$ is not compact modulo $H$, then $(G, H)$ is not of residue type, by Definition 12.1 (a). This establishes (c).

We now turn to (b). Let $f$ be a $W$-invariant even residue weight for $\Sigma$. Then for each $F \subset \Delta$, the parabolic subgroup $P_{F}$ is of residue type so that Proposition 21.1 applies. It now follows from (8.10) combined with 21.1 that 21.3) holds for every $f \in C_{c}^{\infty}(\mathrm{X}: \tau)$. Finally, the validity of (b) follows by density of $C_{c}^{\infty}(\mathrm{X}: \tau)$ and continuity of each of the $\mathcal{J}_{F} \mathcal{F}_{F}$, for $F \subset \Delta$ (Proposition 19.6 and Theorem 20.3 apply with $P=P_{F}$, since $P_{F}$ is of residue type).

It remains to prove (a). We will do this by induction on $\operatorname{dim} A_{\mathrm{q}}$, the $\sigma$ split rank of $G$. First, assume that $\operatorname{dim} A_{\mathrm{q}}=0$. Then $\mathrm{X}$ is compact, hence the pair $(G, H)$ is of residue type, see Remark [12.3 It follows that $G$, viewed as a parabolic subgroup, is of residue type, see Remark 13.3 Since $\mathcal{P}_{\sigma}=\{G\}$, this establishes (a) in case $\operatorname{dim} A_{\mathrm{q}}=0$.

Thus, assume that $\operatorname{dim} A_{\mathrm{q}} \geq 1$ and that (a) has been established for all pairs $\left(G^{\prime}, \sigma^{\prime}\right)$ with $G^{\prime}$ of $\sigma$-split rank smaller than $\operatorname{dim} A_{\mathrm{q}}$.

If the center of $G$ is not compact modulo $H$, then ${ }^{\circ} A_{\mathrm{q}}:={ }^{\circ} G \cap A_{\mathrm{q}} \subsetneq A_{\mathrm{q}}$. Hence, it follows by the inductive hypothesis that every $\sigma$-parabolic subgroup of ${ }^{\circ} G$ containing ${ }^{\circ} A_{\mathrm{q}}$ is of residue type. Denote the set of these parabolic subgroups by ${ }^{\circ} \mathcal{P}_{\sigma}$. Then $G={ }^{\circ} G \times C$, where $C=\exp (\operatorname{center}(\mathfrak{g}) \cap \mathfrak{p})$ and $P \mapsto P C$ is a 
bijection from ${ }^{\circ} \mathcal{P}_{\sigma}$ onto $\mathcal{P}_{\sigma}$. Moreover, $M_{P C}=M_{P}$ for every $P \in \mathcal{P}_{\sigma}\left({ }^{\circ} G\right)$. In view of Definition 13.1 it follows that every parabolic subgroup from $\mathcal{P}_{\sigma}$ is of residue type as well, whence (a).

Thus, we may assume that $G$ has compact center modulo $H$; then $\mathfrak{a}_{\Delta \mathrm{q}}=0$. By the inductive hypothesis, the symmetric pairs $\left(M_{F}, M_{F} \cap v H v^{-1}\right)$, for $F \subsetneq \Delta$ and $v \in N_{K}\left(\mathfrak{a}_{\mathrm{q}}\right)$, satisfy condition (a). In particular, $M_{F}$, viewed as a parabolic subgroup of $M_{F}$, is of residue type relative to $v H v^{-1}$. Since ${ }^{\circ} M_{F}=M_{F}$, it follows by Remark 13.3 that the pairs $\left(M_{F}, M_{F} \cap v H v^{-1}\right)$, are all of residue type. In view of Definition 13.1 it now follows that the standard parabolic subgroups $P_{F}$, for $F \subsetneq \Delta$, are all of residue type.

Let $t$ be a $W$-invariant even residue weight on $\Sigma$. Then $T_{\Delta}^{t}$ is a continuous linear operator from $C_{c}^{\infty}(\mathrm{X}: \tau)$ into the finite dimensional subspace $\mathcal{A}_{\Delta}^{t}(\mathrm{X}: \tau)$ of $C^{\infty}(\mathrm{X}: \tau)$; all functions in this subspace are $\mathbb{D}(\mathrm{X})$-finite, see the text after (9.1).

Proposition 21.1 applies for every subset $F \subsetneq \Delta$. Hence, from (8.10) and (21.1) it follows that

$$
T_{\Delta}^{t}=I-\sum_{F \subsetneq \Delta}\left[W: W_{F}\right] t\left(\mathfrak{a}_{F \mathrm{q}}^{+}\right) \mathcal{J}_{F} \mathcal{F}_{F}
$$

as an operator from $C_{c}^{\infty}(\mathrm{X}: \tau)$ into $C^{\infty}(\mathrm{X}: \tau)$. Applying Proposition 19.6 and Theorem 20.3 we infer that $T_{\Delta}^{t}$ extends to a continuous linear map from $\mathcal{C}(\mathrm{X}: \tau)$ into $\mathcal{A}_{\Delta}^{t}(\mathrm{X}: \tau) \cap \mathcal{C}(\mathrm{X}: \tau)$; moreover, the latter intersection is continuously contained in $\mathcal{A}_{2}(\mathrm{X}: \tau)$. By density of $C_{c}^{\infty}(\mathrm{X}: \tau)$, the validity of the identity (21.4) extends to the space $\mathcal{C}(\mathrm{X}: \tau)$.

By repeated application of Lemma 19.8, with $P=P_{F}, F \subsetneq \Delta$, it follows from (21.4) that $T_{\Delta}^{t}=I$ on $\mathcal{A}_{2}(\mathrm{X}: \tau)$. Finally, by application of Lemma 20.5 to (21.4) it follows that $T_{\Delta}^{t}$ is symmetric with respect to the $L^{2}$-inner product on $\mathcal{C}(\mathrm{X}: \tau)$. We conclude that $T_{\Delta}^{t}$ is the orthogonal projection from $\mathcal{C}(\mathrm{X}: \tau)$ onto $\mathcal{A}_{2}(\mathrm{X}: \tau)$; in particular, it follows that the latter space is finite dimensional. Moreover, since $\mathcal{A}_{2}(\mathrm{X}: \tau)$ is dense in $L_{d}^{2}(\mathrm{X}: \tau)$ it follows that $\mathcal{A}_{2}(\mathrm{X}: \tau)=L_{d}^{2}(\mathrm{X}: \tau)$ and that $T_{\Delta}^{t}$ is the restriction of the orthogonal projection $L^{2}(\mathrm{X}: \tau) \rightarrow L_{d}^{2}(\mathrm{X}: \tau)$. From this we conclude that $(G, H)$ is of residue type, see Definition 12.1 and Remark [12.5. Hence, $P_{\Delta}=G$ is of residue type. It follows that all standard parabolic subgroups in $\mathcal{P}_{\sigma}$ are of residue type. Since every $P \in \mathcal{P}_{\sigma}$ is associated with a standard one, see Lemma 3.6 assertion (a) follows by application of Lemma 13.5

We define the equivalence relation $\sim$ on the collection of subsets of $\Delta$ by $F \sim F^{\prime} \Longleftrightarrow P_{F} \sim P_{F^{\prime}}$.

Lemma 21.3 Let $t$ be a $W$-invariant even residue weight on $\Sigma$ and let $F \subset \Delta$. Then

$$
\sum_{\substack{F^{\prime} \subset \Delta \\ F^{\prime} \sim F}} t\left(\mathfrak{a}_{F^{\prime} \mathrm{q}}^{+}\right)=\left|W\left(\mathfrak{a}_{F \mathrm{q}}\right)\right|^{-1}
$$


Proof: Let $\mathcal{P}_{\sigma}\left(A_{F \mathrm{q}}\right)$ denote the collection of all $P \in \mathcal{P}_{\sigma}$ with $\sigma$-split component $A_{P \mathrm{q}}$ equal to $A_{F \mathrm{q}}$. Moreover, let $\mathcal{S}$ denote the collection of all subsets $F^{\prime} \subset \Delta$ with $F^{\prime} \sim F$. For every $P \in \mathcal{P}_{\sigma}\left(A_{F \mathrm{q}}\right)$ there exists a unique $F_{P} \subset \Delta$ such that $s P s^{-1}=P_{F_{P}}$, for some $s \in W$, see Lemma 3.6 Clearly, $F_{P} \sim F$. Moreover, the map p: $P \mapsto F_{P}$ is surjective from $\mathcal{P}_{\sigma}\left(A_{F q}\right)$ onto $\mathcal{S}$. If $F^{\prime} \in \mathcal{S}$, let $W_{F^{\prime}, F}$ denote the collection of $s \in W$ mapping $\mathfrak{a}_{F q}$ onto $\mathfrak{a}_{F^{\prime} q}$. Then the map $s \mapsto s^{-1} P_{F^{\prime}} s$ from $W_{F^{\prime}, F}$ onto $\mathrm{p}^{-1}\left(F^{\prime}\right)$ factors to a bijection from $W\left(\mathfrak{a}_{F^{\prime} \mathrm{q}} \mid \mathfrak{a}_{F \mathrm{q}}\right)$ onto $\mathrm{p}^{-1}\left(F^{\prime}\right)$. Starting from (2.1) with $Q=P_{F}$ we now obtain that

$$
\begin{aligned}
1 & =\sum_{P \in \mathcal{P}_{\sigma}\left(A_{F \mathfrak{q}}\right)} t\left(\mathfrak{a}_{P \mathfrak{q}}^{+}\right) \\
& =\sum_{\substack{F^{\prime} \subset \Delta \\
F^{\prime} \sim F}} \sum_{s \in W\left(\mathfrak{a}_{F^{\prime}} \mid \mathfrak{a}_{F q}\right)} t\left(s^{-1}\left(\mathfrak{a}_{F^{\prime} \mathrm{q}}^{+}\right)\right) \\
& =\sum_{\substack{F^{\prime} \subset \Delta \\
F^{\prime} \sim F}}\left|W\left(\mathfrak{a}_{F^{\prime} \mathrm{q}} \mid \mathfrak{a}_{F \mathrm{q}}\right)\right| t\left(\mathfrak{a}_{F^{\prime} \mathrm{q}}^{+}\right) .
\end{aligned}
$$

For every $F^{\prime} \subset \Delta$ with $F^{\prime} \sim F$, the group $W\left(\mathfrak{a}_{F q}\right)$ acts freely and transitively from the right on $W\left(\mathfrak{a}_{F^{\prime} \mathbf{q}} \mid \mathfrak{a}_{F \mathrm{q}}\right)$. Hence, $\left|W\left(\mathfrak{a}_{F^{\prime} \mathrm{q}} \mid \mathfrak{a}_{F \mathrm{q}}\right)\right|=\left|W\left(\mathfrak{a}_{F \mathrm{q}}\right)\right|$ and the result follows.

Lemma 21.4 Let $P, Q \in \mathcal{P}_{\sigma}$ be associated parabolic subgroups and let $s \in$ $W\left(\mathfrak{a}_{P \mathrm{q}} \mid \mathfrak{a}_{Q \mathrm{q}}\right)$. Then

(a) $\mathcal{F}_{P} f(s \nu)=C_{P \mid Q}^{\circ}(s: \nu) \mathcal{F}_{Q} f(\nu)$, for all $f \in \mathcal{C}(\mathrm{X}: \tau)$ and $\nu \in i \mathfrak{a}_{Q q}^{*}$;

(b) $\mathcal{J}_{P} \mathcal{F}_{P}=\mathcal{J}_{Q} \mathcal{F}_{Q}$ as endomorphisms of $\mathcal{C}(\mathrm{X}: \tau)$.

Proof: It follows from Corollary 18.2 combined with the Maass-Selberg relations (18.5), that

$$
E^{*}(P: s \nu: x)=C_{P \mid Q}^{\circ}(s: \nu) E^{*}(Q: \nu: x),
$$

for all $x \in \mathrm{X}$ and all $\nu \in i \mathfrak{a}_{Q \mathrm{q}}^{*}$. Now (a) follows by Definition 19.1 The linear bijection $s$ from $i \mathfrak{a}_{Q \mathrm{q}}^{*}$ onto $i \mathfrak{a}_{P \mathrm{q}}^{*}$ maps the chosen Lebesgue measures onto each other, see Section [5] Hence, for $f \in \mathcal{C}(\mathrm{X}: \tau)$,

$$
\mathcal{J}_{P} \mathcal{F}_{P} f(x)=\int_{i \mathfrak{a}_{Q q}^{*}} E^{\circ}(P: s \nu: x) \mathcal{F}_{P} f(s \nu) d \nu
$$

Applying (a) and Proposition 18.1 we obtain that

$$
\begin{aligned}
E^{\circ}(P: s \nu: x) \mathcal{F}_{P} f(s \nu) & =E^{\circ}(P: s \nu: x) C_{P \mid Q}^{\circ}(s: \nu) \mathcal{F}_{Q} f(\nu) \\
& =E^{\circ}(Q: \nu: x) \mathcal{F}_{Q} f(\nu) .
\end{aligned}
$$

Substituting the obtained identity in the right-hand side of (21.5) we obtain (b). 
Remark 21.5 Let $P \in \mathcal{P}_{\sigma}$. Then it follows from part (b) of the above lemma that the continuous linear endomorphism $\mathcal{J}_{P} \circ \mathcal{F}_{P}$ of $\mathcal{C}(\mathrm{X}: \tau)$ depends on $P$ through its equivalence class in $\mathcal{P}_{\sigma} / \sim$.

If $P \in \mathcal{P}_{\sigma}$, we agree to write $W_{P}^{*}$ for the normalizer of $\mathfrak{a}_{P \mathrm{q}}$ in $W$. Then $W\left(\mathfrak{a}_{P \mathrm{q}}\right) \simeq W_{P}^{*} / W_{P}$

Theorem 21.6 Let $f \in \mathcal{C}(\mathrm{X}: \tau)$. Then

$$
f=\sum_{[P] \in \mathcal{P}_{\sigma} / \sim}\left[W: W_{P}^{*}\right] \mathcal{J}_{P} \mathcal{F}_{P} f .
$$

Remark 21.7 In view of Remark 13.10 this theorem corresponds to part (iii) of Thm. 2 in Delorme's paper [24. Note that in the latter theorem constants $\left|W\left(\mathfrak{a}_{P \mathrm{q}}\right)\right|^{-1}$, for $P \in \mathcal{P}_{\sigma}$, appear in place of the constants $\left[W: W_{P}^{*}\right]$. This is due to a different normalization of measures, as will be explained in the sequel [15] to this paper.

Proof: We observe that $\left[W: W_{P}\right]\left|W\left(\mathfrak{a}_{P q}\right)\right|^{-1}=\left[W: W_{P}^{*}\right]$, for $P \in \mathcal{P}_{\sigma}$. Since every $P \in \mathcal{P}_{\sigma}$ is associated with a standard parabolic subgroup, see Lemma 3.6 the result now follows from Theorem 21.2] Lemma 21.3] and Remark 21.5

\section{Properties of the Fourier transforms}

The purpose of this section is to establish relations between the different Fourier and wave packet transforms $\mathcal{F}_{P}$ and $\mathcal{J}_{Q}$, as $P, Q \in \mathcal{P}_{\sigma}$. We shall also determine the image of $\mathcal{F}_{P}$ and the kernel of $\mathcal{J}_{Q}$. The relation between the several Fourier transforms is given by Lemma 21.4 (a).

Lemma 22.1 Let $P, Q, R \in \mathcal{P}_{\sigma}$ be associated. Then, for all $s \in W\left(\mathfrak{a}_{Q \mathrm{q}} \mid \mathfrak{a}_{P \mathrm{q}}\right)$ and $t \in W\left(\mathfrak{a}_{R \mathrm{q}} \mid \mathfrak{a}_{Q \mathrm{q}}\right)$,

$$
C_{R \mid P}^{\circ}(t s: \nu)=C_{R \mid Q}^{\circ}(t: s \nu) \circ C_{Q \mid P}^{\circ}(s: \nu), \quad\left(\nu \in i \mathfrak{a}_{P \mathrm{q}}^{*}\right) .
$$

Proof: The above identity is an immediate consequence of the functional equation for the Eisenstein integral, see Proposition 18.1 and the definition of the $C$-function, see Definition 17.6

In particular, from the above lemma with $P=Q=R$ combined with the Maass-Selberg relations, see Theorem 18.3 we see that we may define a unitary representation $\gamma_{P}$ of $W\left(\mathfrak{a}_{P \mathrm{q}}\right)$ in $L^{2}\left(i \mathfrak{a}_{P \mathrm{q}}^{*}\right) \otimes \mathcal{A}_{2, P}$ by

$$
\left[\gamma_{P}(s) \varphi\right](\nu)=C_{P \mid P}^{\circ}\left(s: s^{-1} \nu\right) \varphi\left(s^{-1} \nu\right), \quad\left(\nu \in i \mathfrak{a}_{P \mathrm{q}}^{*}\right),
$$

for $\varphi \in L^{2}\left(i \mathfrak{a}_{P \mathrm{q}}^{*}\right) \otimes \mathcal{A}_{2, P}$. The associated collection of $W\left(\mathfrak{a}_{P \mathrm{q}}\right)$-invariants in $L^{2}\left(i \mathfrak{a}_{P \mathrm{q}}^{*}\right) \otimes \mathcal{A}_{2, P}$ is denoted by $\left(L^{2}\left(i \mathfrak{a}_{P \mathrm{q}}^{*}\right) \otimes \mathcal{A}_{2, P}\right)^{W\left(\mathfrak{a}_{P \mathrm{q}}\right)}$. The orthogonal projection from the first onto the latter space is denoted by

$$
\mathrm{P}_{W\left(\mathfrak{a}_{P \mathrm{q}}\right)}: \quad L^{2}\left(i \mathfrak{a}_{P \mathrm{q}}^{*}\right) \otimes \mathcal{A}_{2, P} \rightarrow\left(L^{2}\left(i \mathfrak{a}_{P \mathrm{q}}^{*}\right) \otimes \mathcal{A}_{2, P}\right)^{W\left(\mathfrak{a}_{P \mathrm{q}}\right)}
$$


The intersection of the latter space with $\mathcal{S}\left(i \mathfrak{a}_{P q}^{*}\right) \otimes \mathcal{A}_{2, P}$ consists of the functions $\varphi \in \mathcal{S}\left(i \mathfrak{a}_{P q}^{*}\right) \otimes \mathcal{A}_{2, P}$ satisfying

$$
\varphi(s \nu)=C_{P \mid P}^{\circ}(s: \nu) \varphi(\nu), \quad\left(s \in W\left(\mathfrak{a}_{P \mathrm{q}}\right), \nu \in i \mathfrak{a}_{P \mathrm{q}}^{*}\right),
$$

and is denoted by $\left(\mathcal{S}\left(i \mathfrak{a}_{P \mathrm{q}}^{*}\right) \otimes \mathcal{A}_{2, P}\right)^{W\left(\mathfrak{a}_{P \mathrm{q}}\right)}$.

Corollary 22.2 Let $P \in \mathcal{P}_{\sigma}$. The image of $\mathcal{C}(\mathrm{X}: \tau)$ under the Fourier transform $\mathcal{F}_{P}$ is contained in the space $\left(\mathcal{S}\left(i \mathfrak{a}_{P \mathrm{q}}^{*}\right) \otimes \mathcal{A}_{2, P}\right)^{W\left(\mathfrak{a}_{P q}\right)}$.

Proof: Let $f \in \mathcal{C}(\mathrm{X}: \tau)$. Then it follows from Lemma 21.4(a) with $P=Q$ that $\varphi:=\mathcal{F}_{P} f$ satisfies (22.1).

We can now state the first main result of this section.

Theorem 22.3 Let $P, Q \in \mathcal{P}_{\sigma}$.

(a) If $P$ and $Q$ are not associated, then $\mathcal{F}_{Q} \circ \mathcal{J}_{P}=0$.

(b) If $P$ and $Q$ are associated, then $\left[W: W_{P}^{*}\right] \mathcal{F}_{Q} \circ \mathcal{J}_{P} \circ \mathcal{F}_{P}=\mathcal{F}_{Q}$ on $\mathcal{C}(\mathrm{X}: \tau)$.

(c) If $P$ and $Q$ are associated, then, for each $s \in W\left(\mathfrak{a}_{Q \mathrm{q}} \mid \mathfrak{a}_{P \mathrm{q}}\right)$, every $\varphi \in$ $\mathcal{S}\left(i \mathfrak{a}_{P \mathrm{q}}^{*}\right) \otimes \mathcal{A}_{2, P}$ and all $\nu \in i \mathfrak{a}_{P \mathrm{q}}^{*}$

$$
\mathcal{F}_{Q} \circ \mathcal{J}_{P} \varphi(s \nu)=\left[W: W_{P}^{*}\right]^{-1} C_{Q \mid P}^{\circ}(s: \nu) \mathrm{P}_{W\left(\mathfrak{a}_{P q}\right)} \varphi(\nu) .
$$

In particular, $\mathcal{F}_{P} \circ \mathcal{J}_{P}=\left[W: W_{P}^{*}\right]^{-1} \mathrm{P}_{W\left(\mathfrak{a}_{P q}\right)}$.

The proof is analogous to the proof of Theorem 16.6 in 10, with adaptations to deal with the present more general situation. In the course of the proof we need two lemmas. The first of these is a straightforward generalization of Lemma 16.11 in [10].

Lemma 22.4 Let $P \in \mathcal{P}_{\sigma}$, let $\mathfrak{b} \subset \mathfrak{q}$ be a $\theta$-stable Cartan subspace containing $\mathfrak{a}_{P \mathrm{q}}$ and let $\Lambda \in{ }^{*} \mathfrak{b}_{P \mathbb{C}}^{*}$. Then for $\lambda$ in the complement of a finite union of affine hyperplanes in $\mathfrak{a}_{P \mathrm{qc}}^{*}$, the map $D \mapsto d[\gamma(D: \Lambda+\cdot)](\lambda)$ is surjective from $\mathbb{D}(\mathrm{X})$ onto $\mathfrak{a}_{P \mathrm{qc}}$.

Proof: The proof is a straightforward modification of the proof of Lemma 16.12 in [10. In that proof one should everywhere put $\mathfrak{a}_{P \mathrm{q}}$ in place of $\mathfrak{a}_{\mathrm{q}}$ and ${ }^{*} \mathfrak{b}_{P}$ in place of $\mathfrak{b}_{\mathrm{k}}$. In particular, $\pi_{\mathfrak{a}_{\mathrm{q}}}$ should be replaced by the projection $\pi_{\mathfrak{a}_{P \mathrm{q}}}: \mathfrak{b}_{\mathbb{C}}^{*} \rightarrow \mathfrak{a}_{P \mathrm{qC}}^{*}$ along the subspace ${ }^{*} \mathfrak{b}_{P \mathbb{C}}^{*}$.

The next lemma is a consequence of Lemma 16.4 which in turn heavily relies on the information about the infinitesimal characters of discrete series stated in Theorem [16.1.

Lemma 22.5 Let $P, Q \in \mathcal{P}_{\sigma}$, let $\mathfrak{b} \subset \mathfrak{q}$ be a $\theta$-stable Cartan subspace containing $\mathfrak{a}_{\mathrm{q}}$ and let $\Lambda_{1} \in \mathrm{L}_{P}(\mathfrak{b}, \tau)$ and $\Lambda_{2} \in \mathrm{L}_{Q}(\mathfrak{b}, \tau)$. Let $\nu_{1} \in i \mathfrak{a}_{P \mathrm{q}}^{*}$ be such that $\Lambda_{1}+\nu_{1}$ is regular with respect to $\Sigma(\mathfrak{b})$, let $\nu_{2} \in i \mathfrak{a}_{Q q}^{*}$ and assume that $\Lambda_{1}+\nu_{1}$ and $\Lambda_{2}+\nu_{2}$ are conjugate under $W(\mathfrak{b})$. Then $P$ and $Q$ are associated, and $\nu_{1}$ and $\nu_{2}$ are conjugate under $W\left(\mathfrak{a}_{Q \mathrm{q}} \mid \mathfrak{a}_{P \mathrm{q}}\right)$. 
Proof: Let $s \in W(\mathfrak{b})$ be such that $s\left(\Lambda_{1}+\nu_{1}\right)=\Lambda_{2}+\nu_{2}$. Select $v_{1} \in{ }^{P} \mathcal{W}$ and $v_{2} \in Q_{\mathcal{W}}$ such that $\Lambda_{1} \in L_{P, v_{1}}(\mathfrak{b}, \tau)$ and $\Lambda_{2} \in L_{Q, v_{2}}(\mathfrak{b}, \tau)$. Let $\left(\hat{\mathfrak{b}}_{1}, t_{1}\right)$ and $\left(\hat{\mathfrak{b}}_{2}, t_{2}\right)$ be associated with $P, v_{1}$ and $Q, v_{2}$, respectively, as in Lemma 16.4. Then $t_{1} \Lambda_{1}+t_{1} \nu_{1}$ and $t_{2} \Lambda_{2}+t_{2} \nu_{2}$ are conjugate under $t_{2} s t_{1}^{-1} \in W\left(\hat{\mathfrak{b}}_{2} \mid \hat{\mathfrak{b}}_{1}\right)$. It follows by application of [17, Lemma 2, that $t_{1} \mathfrak{a}_{P \mathrm{q}}=\operatorname{Ad}\left(v_{1}\right)^{-1} \mathfrak{a}_{P \mathrm{q}}$ and $t_{2} \mathfrak{a}_{Q \mathrm{q}}=\operatorname{Ad}\left(v_{2}\right)^{-1} \mathfrak{a}_{Q \mathrm{q}}$ are conjugate under $t_{2} s t_{1}^{-1}$. This implies that $s\left(\mathfrak{a}_{P \mathrm{q}}\right)=$ $\mathfrak{a}_{Q \mathrm{q}}$. It follows that $\left.s\right|_{\mathfrak{a}_{P \mathrm{q}}} \in W\left(\mathfrak{a}_{Q \mathrm{q}} \mid \mathfrak{a}_{P \mathrm{q}}\right)$, see Lemma 3.7 hence, $P \sim Q$. It also follows by [17, Lemma 2, that $t_{2} s t_{1}^{-1}$ maps $t_{1} \nu_{1}$ onto $t_{2} \nu_{2}$; hence, $s \nu_{1}=\nu_{2}$.

The following lemma collects some properties of the composition $\mathcal{F}_{Q} \circ \mathcal{J}_{P}$ needed in the proof of Theorem 22.3

Lemma 22.6 Let $P, Q \in \mathcal{P}_{\sigma}$. Then the composition $\mathcal{T}:=\mathcal{F}_{Q} \circ \mathcal{J}_{P}$ is a continuous linear map from $\mathcal{S}\left(i \mathfrak{a}_{P \mathrm{q}}^{*}\right) \otimes \mathcal{A}_{2, P}$ to $\mathcal{S}\left(i \mathfrak{a}_{Q \mathrm{q}}^{*}\right) \otimes \mathcal{A}_{2, Q}$. Moreover, it satisfies the following properties.

(a) $\underline{\mu}_{Q}(D) \circ \mathcal{T}=\mathcal{T} \circ \underline{\mu}_{P}(D)$ for all $D \in \mathbb{D}(\mathrm{X})$.

(b) $\mathcal{T}$ maps into $\left(\mathcal{S}\left(i \mathfrak{a}_{Q \mathrm{q}}^{*}\right) \otimes \mathcal{A}_{2, Q}\right)^{W\left(\mathfrak{a}_{Q \mathrm{q}}\right)}$.

Proof: The continuity of $\mathcal{T}$ follows from Theorem 20.3 combined with Proposition 19.6 Property (a) follows from Lemma 20.4 combined with Lemma 19.4. Finally, (b) follows from Corollary 22.2

Proposition 22.7 Let $P, Q \in \mathcal{P}_{\sigma}$. There exists an open dense $W\left(\mathfrak{a}_{Q \mathrm{q}}\right)$-invariant subset $\Omega \subset i \mathfrak{a}_{Q \mathrm{q}}^{*}$ with the following property. Let $\mathcal{T}$ be any continuous linear map from $\mathcal{S}\left(i \mathfrak{a}_{P \mathrm{q}}^{*}\right) \otimes \mathcal{A}_{2, P}$ to $\mathcal{S}\left(i \mathfrak{a}_{Q \mathrm{q}}^{*}\right) \otimes \mathcal{A}_{2, Q}$ satisfying the properties of Lemma 22.6.

(a) If $P$ and $Q$ are not associated, then $\mathcal{T}=0$.

(b) If $P$ and $Q$ are associated and $s_{0} \in W\left(\mathfrak{a}_{P \mathrm{q}} \mid \mathfrak{a}_{Q \mathrm{q}}\right)$, then there exists a unique smooth function $\beta: \Omega \rightarrow \operatorname{Hom}\left(\mathcal{A}_{2, P}, \mathcal{A}_{2, Q}\right)$ such that

$$
\mathcal{T} \varphi(\nu)=\mathrm{P}_{W\left(\mathfrak{a}_{Q \mathrm{q}}\right)}\left(\beta s_{0}^{*} \varphi\right)(\nu),
$$

for all $\varphi \in C_{c}^{\infty}\left(s_{0} \Omega\right) \otimes \mathcal{A}_{2, P}$ and $\nu \in \Omega$.

Proof: For every $\nu \in i \mathfrak{a}_{Q \mathrm{q}}^{*}$, we define the distribution $u_{\nu} \in \mathcal{D}^{\prime}\left(i \mathfrak{a}_{P \mathrm{q}}^{*}\right) \otimes$ $\operatorname{Hom}\left(\mathcal{A}_{2, P}, \mathcal{A}_{2, Q}\right)$ by

$$
u_{\nu}(\varphi)=\mathcal{T}(\varphi)(\nu), \quad\left(\varphi \in C_{c}^{\infty}\left(i \mathfrak{a}_{P \mathrm{q}}^{*}\right) \otimes \mathcal{A}_{2, P}\right) .
$$

Then it follows from condition (a) that

$$
u_{\nu} \circ \underline{\mu}_{P}(D)=\underline{\mu}_{Q}(D: \nu) u_{\nu}, \quad(D \in \mathbb{D}(\mathrm{X})) .
$$


Let now $\Lambda_{1} \in \mathrm{L}_{P}(\mathfrak{b}, \tau)$ and $\psi_{1} \in \mathcal{A}_{2, P}\left(\Lambda_{1}\right)$. Let $\Lambda_{2} \in \mathrm{L}_{Q}(\mathfrak{b}, \tau)$ and $\psi_{2} \in$ $\mathcal{A}_{2, Q}\left(\Lambda_{2}\right)$. We define the distribution $v_{\nu} \in \mathcal{D}^{\prime}\left(i \mathfrak{a}_{P \mathrm{q}}^{*}\right)$ by $v_{\nu}(f)=\left\langle u_{\nu}\left(f \otimes \psi_{1}\right) \mid \psi_{2}\right\rangle$, for $f \in C_{c}^{\infty}\left(i \mathfrak{a}_{P q}^{*}\right)$. It follows that

$$
\left[\gamma_{\mathfrak{b}}\left(D: \Lambda_{1}+\cdot\right)-\gamma_{\mathfrak{b}}\left(D: \Lambda_{2}+\nu\right)\right] v_{\nu}=0 .
$$

Each element $\Lambda$ from the finite set $\mathrm{L}_{Q}(\mathfrak{b}, \tau)$ is regular with respect to $\Sigma\left(\mathfrak{m}_{Q \mathbb{C}},{ }^{*} \mathfrak{b}_{Q}\right)$, see Corollary 16.6] Let $\Omega_{0}$ be the set of $\nu \in i \mathfrak{a}_{Q q}^{*}$ such that $\Lambda+\nu$ is regular with respect to $\Sigma(\mathfrak{b})$, for every $\Lambda \in \mathrm{L}_{Q}(\mathfrak{b}, \tau)$. Then $\Omega_{0}$ is the complement of a finite union of hyperplanes in $i \mathfrak{a}_{Q \mathrm{q}}^{*}$, hence open dense.

Let $\nu \in \Omega_{0}$ and let $\Lambda_{1}, \Lambda_{2}$ be as above. Moreover, let $\nu_{1} \in i \mathfrak{a}_{P \mathrm{q}}^{*}$ and assume that $\nu \notin W\left(\mathfrak{a}_{Q_{\mathrm{q}}} \mid \mathfrak{a}_{P \mathrm{q}}\right) \nu_{1}$. Then by Lemma 22.5 the elements $\Lambda_{1}+\nu_{1}$ and $\Lambda_{2}+\nu$ are not conjugate under $W(\mathfrak{b})$. Hence, there exists a $D \in \mathbb{D}(\mathrm{X})$ such that the polynomial function in front of $v_{\nu}$ in 22.3) does not vanish at $\nu_{1}$. This implies that $v_{\nu}$ vanishes in a neighborhood of $\nu_{1}$. Let $\varphi \in C_{c}^{\infty}\left(i \mathfrak{a}_{P \mathrm{q}}^{*}\right) \otimes \mathcal{A}_{2, P}$. Then it follows from the above by linearity that $\mathcal{T}(\varphi)(\nu)=u_{\nu}(\varphi)=0$ for all $\nu \in \Omega_{0}$ with $\nu \notin W\left(\mathfrak{a}_{Q \mathrm{q}} \mid \mathfrak{a}_{P \mathrm{q}}\right) \operatorname{supp} \varphi$. By density and continuity, this implies that the function $\mathcal{T}(\varphi)$ vanishes on $i \mathfrak{a}_{Q \mathrm{q}}^{*} \backslash W\left(\mathfrak{a}_{Q \mathrm{q}} \mid \mathfrak{a}_{P \mathrm{q}}\right) \operatorname{supp} \varphi$. Hence,

$$
\operatorname{supp} \mathcal{T} \varphi \subset W\left(\mathfrak{a}_{Q \mathrm{q}} \mid \mathfrak{a}_{P \mathrm{q}}\right) \operatorname{supp} \varphi
$$

If $P$ and $Q$ are not associated, then it follows that the latter set has empty interior in $i \mathfrak{a}_{Q \mathrm{q}}^{*}$, hence $\mathcal{T} \varphi=0$ by continuity. This establishes (a).

From now on, we assume that $P \sim Q$. Then it follows from the above that

$$
\operatorname{supp} u_{\nu} \subset W\left(\mathfrak{a}_{P \mathrm{q}} \mid \mathfrak{a}_{Q \mathrm{q}}\right) \nu,
$$

for every $\nu \in i \mathfrak{a}_{Q q}^{*}$.

Let $\Omega_{1}$ be the set of $\nu \in i \mathfrak{a}_{Q \mathrm{q}}^{*}$ whose stabilizer in $W\left(\mathfrak{a}_{Q \mathrm{q}}\right)$ is trivial. Then $\Omega_{1}$ contains the complement of a finite union of hyperplanes in $i \mathfrak{a}_{Q q}^{*}$ hence is open dense in $i \mathfrak{a}_{Q \mathrm{q}}^{*}$. Since $W\left(\mathfrak{a}_{Q \mathrm{q}}\right)$ acts simply transitively on $W\left(\mathfrak{a}_{P \mathrm{q}} \mid \mathfrak{a}_{Q \mathrm{q}}\right)$ from the right, we see that if $\nu \in \Omega_{1}$, the points $s \nu$, for $s \in W\left(\mathfrak{a}_{P \mathrm{q}} \mid \mathfrak{a}_{Q \mathrm{q}}\right)$, are mutually different.

Let $\Omega_{2}^{\prime}$ be the set of $\mu \in i \mathfrak{a}_{P \mathrm{q}}^{*}$ such that for every $\Lambda_{1} \in \mathrm{L}_{P}(\mathfrak{b}, \tau)$ the map $D \mapsto d\left[\gamma_{\mathfrak{b}}\left(D: \Lambda_{1}+\cdot\right)\right](\mu)$ is surjective from $\mathbb{D}(\mathrm{X})$ onto $\mathfrak{a}_{P \mathrm{qc}}^{*}$. Then $\Omega_{2}^{\prime}$ is an open subset of $i \mathfrak{a}_{P \mathrm{q}}^{*}$ containing the complement of a finite union of hyperplanes, see Lemma 22.4 It follows that $\Omega_{2}=\cap_{s \in W\left(\mathfrak{a}_{P q} \mid \mathfrak{a}_{Q q}\right)} s^{-1} \Omega_{2}^{\prime}$ is a similar subset of $i \mathfrak{a}_{Q q}^{*}$. We define $\Omega:=\Omega_{1} \cap \Omega_{2}$.

Let now $\nu \in \Omega$. We claim that the distribution $u_{\nu}$ has order zero. To prove the claim, fix $\Lambda_{1}, \Lambda_{2}, \psi_{1}, \psi_{2}$ as before, and define $v=v_{\nu}$ as above. Then by linearity, it suffices to show that $v$ has order zero. Since $\operatorname{supp}(v) \subset W\left(\mathfrak{a}_{P q} \mid\right.$ $\left.\mathfrak{a}_{Q \mathrm{q}}\right) \nu$, by (22.4), it follows by our assumption on $\Omega_{1}$ that we may express $v$ uniquely as a sum of distributions $v_{s}$, for $s \in W\left(\mathfrak{a}_{P \mathrm{q}} \mid \mathfrak{a}_{Q \mathrm{q}}\right)$, with $\operatorname{supp} v_{s} \subset\{s \nu\}$. From (22.3) it follows that each $v_{s}$ satisfies the equations

$$
\varphi_{D} v_{s}=0, \quad(D \in \mathbb{D}(\mathrm{X}))
$$

where $\varphi_{D}: i \mathfrak{a}_{P \mathfrak{q}}^{*} \rightarrow \mathbb{C}$ is given by $\varphi_{D}=\gamma_{\mathfrak{b}}\left(D: \Lambda_{1}+\cdot\right)-\gamma_{\mathfrak{b}}(D: \Lambda+\nu)$. 
It follows from our assumption on $\Omega_{2}$ that the collection of differentials $d \varphi_{D}(s \nu)$, for $D \in \mathbb{D}(\mathrm{X})$, spans $\mathfrak{a}_{P \mathrm{qC}}^{*}$. Now apply [10], Lemma 16.10 , to conclude that $v_{s}$ has order zero, for each $s \in W\left(\mathfrak{a}_{P \mathrm{q}} \mid \mathfrak{a}_{Q \mathrm{q}}\right)$. This establishes the claim that $v_{\nu}$ has order zero.

It also follows from the above that

$$
u_{\nu}=\sum_{s \in W\left(\mathfrak{a}_{P \mathrm{q}} \mid \mathfrak{a}_{Q \mathrm{q}}\right)} \delta_{s \nu} \otimes E_{s}(\nu)
$$

with $E_{s}(\nu)$ a unique element of $\operatorname{Hom}\left(\mathcal{A}_{2, P}, \mathcal{A}_{2, Q}\right)$, for $s \in W\left(\mathfrak{a}_{P \mathrm{q}} \mid \mathfrak{a}_{Q \mathrm{q}}\right)$. We conclude that, for every $\varphi \in C_{c}^{\infty}\left(i \mathfrak{a}_{P q}^{*}\right) \otimes \mathcal{A}_{2, P}$ and all $\nu \in \Omega$,

$$
\mathcal{T} \varphi(\nu)=\sum_{s \in W\left(\mathfrak{a}_{P \mathrm{q}} \mid \mathfrak{a}_{Q \mathrm{q}}\right)} E_{s}(\nu) \varphi(s \nu) .
$$

Fix $s_{0} \in W\left(\mathfrak{a}_{P \mathrm{q}} \mid \mathfrak{a}_{Q \mathrm{q}}\right)$. Let $\nu_{0} \in \Omega$. By the assumption on $\Omega_{1}$ there exists an open neighborhood $U$ of $\nu_{0}$ in $\Omega$ such that the sets $s U$ are mutually disjoint, for $s \in W\left(\mathfrak{a}_{P \mathrm{q}} \mid \mathfrak{a}_{Q \mathrm{q}}\right)$. For $\varphi \in C_{c}^{\infty}\left(s_{0} U\right) \otimes \mathcal{A}_{2, P}$ we have

$$
\mathcal{T}(\varphi)(\nu)=E_{s_{0}}(\nu) \varphi\left(s_{0} \nu\right) .
$$

We conclude that $E_{s_{0}}$ is smooth on $U$. It follows that $E_{s_{0}} \in C^{\infty}(\Omega) \otimes \operatorname{Hom}\left(\mathcal{A}_{2, P}, \mathcal{A}_{2, Q}\right)$. From the above asserted uniqueness of the $E_{s}$ and the transformation property of $\mathcal{T} \varphi$ stated in Lemma 22.6 (b), it follows that

$$
E_{s_{0}}(t \nu)=C_{Q \mid Q}^{\circ}(t: \nu) E_{s_{0}}(\nu), \quad\left(t \in W\left(\mathfrak{a}_{Q \mathrm{q}}\right)\right) .
$$

If we combine this with (22.5) we obtain, for all $\varphi \in C_{c}^{\infty}\left(s_{0} \Omega\right) \otimes \mathcal{A}_{2, P}$, and all $\nu \in \Omega$, that

$\mathcal{T} \varphi(\nu)=\sum_{t \in W\left(\mathfrak{a}_{Q \mathrm{q}}\right)} C_{Q \mid Q}^{\circ}(t: \nu)^{-1} E_{s_{0}}(t \nu) \varphi\left(s_{0} t \nu\right)=\left|W\left(\mathfrak{a}_{Q \mathrm{q}}\right)\right| \mathrm{P}_{W\left(\mathfrak{a}_{Q \mathrm{q}}\right)}\left(E_{s_{0}} s_{0}^{*} \varphi\right)(\nu)$.

This establishes the result with $\beta=\left|W\left(\mathfrak{a}_{Q \mathrm{q}}\right)\right| E_{s_{0}}$.

Proof of Theorem 22.3; If $P \nsim Q$, then it follows from Lemma 22.6] combined with Proposition 22.7 that $\mathcal{F}_{Q} \circ \mathcal{J}_{P}=0$.

To prove (b) we note that if $f \in C_{c}^{\infty}(\mathrm{X}: \tau)$, then it follows from Theorem 21.6] that $f=\sum_{[R] \in \mathcal{P}_{\sigma} / \sim}\left[W: W_{R}^{*}\right] \mathcal{J}_{R} \mathcal{F}_{R} f$. Applying $\mathcal{F}_{Q}$ to both sides of this equation, it follows by (a) that

$$
\mathcal{F}_{Q} f=\left[W: W_{R}^{*}\right] \mathcal{F}_{Q} \mathcal{J}_{R} \mathcal{F}_{R} f
$$

for each $R \in \mathcal{P}_{\sigma}$ with $R \sim Q$; in particular, we may take $R=P$. Thus, (b) follows by continuity and density.

We shall first prove (c) under the assumption that $P=Q$ and $s=1$. Put $\mathcal{T}=\mathcal{F}_{P} \circ \mathcal{J}_{P}$. From Lemma 22.6 and Proposition 22.7 we deduce that

$$
\mathcal{T} \psi(\nu)=\mathrm{P}_{W\left(\mathfrak{a}_{P \mathrm{q}}\right)}(\beta \psi)(\nu),
$$


for all $\psi \in C_{c}^{\infty}(\Omega) \otimes \mathcal{A}_{2, P}$ and $\nu \in \Omega$; here $\Omega \subset i \mathfrak{a}_{P \mathrm{q}}^{*}$ is an open dense subset, and $\beta \in C^{\infty}(\Omega) \otimes \operatorname{End}\left(\mathcal{A}_{2, P}\right)$. It follows from Lemma 20.5 that the operator $\mathcal{T}$ is symmetric with respect to the $L^{2}$-inner product on $\mathcal{S}\left(i \mathfrak{a}_{P \mathrm{q}}\right) \otimes \mathcal{A}_{2, P}$. Let $\varphi \in \mathcal{S}\left(i \mathfrak{a}_{P \mathrm{q}}\right) \otimes \mathcal{A}_{2, P}$. Then for all $\psi \in C_{c}^{\infty}(\Omega) \otimes \mathcal{A}_{2, P}$,

$$
\langle\mathcal{T} \varphi \mid \psi\rangle=\langle\varphi \mid \mathcal{T} \psi\rangle=\left\langle\varphi \mid \mathrm{P}_{W\left(\mathfrak{a}_{P q}\right)}(\beta \psi)\right\rangle=\left\langle\beta^{*} \mathrm{P}_{W\left(\mathfrak{a}_{P q}\right)} \varphi \mid \psi\right\rangle .
$$

This implies that, for all $\varphi \in \mathcal{S}\left(i \mathfrak{a}_{P \mathrm{q}}^{*}\right) \otimes \mathcal{A}_{2, P}, \mathcal{T} \varphi=\beta^{*} \mathrm{P}_{W\left(\mathfrak{a}_{P \mathrm{q}}\right)} \varphi$ on $\Omega$. We claim that in fact $\beta^{*}=\left[W: W_{P}^{*}\right]^{-1} I$ on $\Omega$.

To prove the claim we note that it follows from the established part (b) with $P=Q$ that $\mathcal{F}_{P}=\left[W: W_{P}^{*}\right] \mathcal{T F}_{P}$. We infer that, for $f \in C_{c}^{\infty}(\mathrm{X}: \tau)$ and $\nu \in \Omega$,

$$
\begin{aligned}
\mathcal{F}_{P} f(\nu) & =\left[W: W_{P}^{*}\right] \mathcal{T} \mathcal{F}_{P} f(\nu) \\
& =\left[W: W_{P}^{*}\right] \beta(\nu)^{*} \mathrm{P}_{W\left(\mathfrak{a}_{P \mathrm{q}}\right)} \mathcal{F}_{P} f(\nu) \\
& =\left[W: W_{P}^{*}\right] \beta(\nu)^{*} \mathcal{F}_{P} f(\nu) .
\end{aligned}
$$

In the last equality we have used Corollary 22.2 The claim now follows by application of Lemma 22.8 below. We infer that for all $\varphi \in \mathcal{S}\left(i \mathfrak{a}_{P \mathrm{q}}\right) \otimes \mathcal{A}_{2, P}$, we have $\mathcal{T} \varphi=\left[W: W_{P}^{*}\right]^{-1} \mathrm{P}_{W\left(\mathfrak{a}_{P \mathrm{q}}\right)} \varphi$ on $\Omega$, hence on $i \mathfrak{a}_{P \mathrm{q}}^{*}$, by continuity and density. This establishes part (c) of the theorem for $P=Q$ and $s=1$. For a general pair of associated parabolic subgroups $P, Q$ and a general $s \in W\left(\mathfrak{a}_{P q} \mid\right.$ $\mathfrak{a}_{Q \mathrm{q}}$ ) the assertion follows by application of Lemma 21.4 (a).

Lemma 22.8 Let $P \in \mathcal{P}_{\sigma}$ and let $\nu \in i \mathfrak{a}_{P \mathrm{q}}^{*}$ have trivial stabilizer in $W\left(\mathfrak{a}_{P \mathrm{q}}\right)$. Then $f \mapsto \mathcal{F}_{P} f(\nu)$ maps $C_{c}^{\infty}(\mathrm{X}: \tau)$ onto $\mathcal{A}_{2, P}$.

Proof: The proof is a reduction to the lemma below, in a way completely analogous to the proof of [10], Lemma 16.13.

Lemma 22.9 Let $P \in \mathcal{P}_{\sigma}$ and let $\nu \in i \mathfrak{a}_{P \mathrm{q}}^{*}$ have trivial stabilizer in $W\left(\mathfrak{a}_{P \mathrm{q}}\right)$. Then for every $\psi \in \mathcal{A}_{2, P} \backslash\{0\}$, the Eisenstein integral $E^{\circ}(P: \nu) \psi$ does not vanish identically on $\mathrm{X}$.

Proof: The proof is completely analogous to the proof of [10], Lemma 16.14, involving the constant term of the Eisenstein integral along $P$.

Proposition 22.10 Let $P \in \mathcal{P}_{\sigma}$.

(a) The map $\mathrm{P}_{W\left(\mathfrak{a}_{P q}\right)}$ restricts to a continuous linear projection from $\mathcal{S}\left(i \mathfrak{a}_{P q}^{*}\right) \otimes$ $\mathcal{A}_{2, P}$ onto $\left(\mathcal{S}\left(i \mathfrak{a}_{P \mathrm{q}}^{*}\right) \otimes \mathcal{A}_{2, P}\right)^{W\left(\mathfrak{a}_{P q}\right)}$. This projection is orthogonal with respect to the given $L^{2}$-inner product.

(b) $\mathcal{J}_{P} \circ \mathrm{P}_{W\left(\mathfrak{a}_{P \mathrm{q}}\right)}=\mathcal{J}_{P}$.

(c) The kernel of $\mathcal{J}_{P}$ equals the kernel of $\mathrm{P}_{W\left(\mathfrak{a}_{P q}\right)}$. 
Proof: It follows from Theorem 22.3 (c) that $\mathrm{P}_{W\left(\mathfrak{a}_{P \mathrm{q}}\right)}=\left[W: W_{P}^{*}\right] \mathcal{F}_{P} \circ \mathcal{J}_{P}$. It now follows from application of Lemma 22.6 that $\mathrm{P}_{W\left(\mathfrak{a}_{P q}\right)}$ is a continuous linear endomorphism of $\mathcal{S}\left(i \mathfrak{a}_{P \mathrm{q}}^{*}\right) \otimes \mathcal{A}_{2, P}$, with image contained in $\left(\mathcal{S}\left(i \mathfrak{a}_{P \mathrm{q}}^{*}\right) \otimes\right.$ $\left.\mathcal{A}_{2, P}\right)^{W\left(\mathfrak{a}_{P q}\right)}$. The latter space is contained in $\left(L^{2}\left(i \mathfrak{a}_{P \mathrm{q}}^{*}\right) \otimes \mathcal{A}_{2, P}\right)^{W\left(\mathfrak{a}_{P q}\right)}$, on which space $\mathrm{P}_{W\left(\mathfrak{a}_{P q}\right)}$ equals the identity. Hence, $\mathrm{P}_{W\left(\mathfrak{a}_{P q}\right)}$ is a projection and (a) readily follows.

Starting from Theorem 22.3 (b) with $P=Q$, we obtain by taking adjoints and applying Lemma 20.5 that

$$
\mathcal{J}_{P} \circ\left[W: W_{P}^{*}\right] \mathcal{F}_{P} \circ \mathcal{J}_{P}=\mathcal{J}_{P} .
$$

Assertion (b) now follows by application of Theorem 22.3 (c).

From (b) it follows that ker $\mathrm{P}_{W\left(\mathfrak{a}_{P q}\right)} \subset$ ker $\mathcal{J}_{P}$. The converse inclusion follows by Theorem 22.3 (c).

Proposition 22.11 Let $P \in \mathcal{P}_{\sigma}$. Then the image of $\mathcal{F}_{P}$ equals $\left(\mathcal{S}\left(i \mathfrak{a}_{P \mathrm{q}}^{*}\right) \otimes\right.$ $\left.\mathcal{A}_{2, P}\right)^{W\left(\mathfrak{a}_{P \mathrm{q}}\right)}$.

Proof: That $\operatorname{im}\left(\mathcal{F}_{P}\right) \subset\left(\mathcal{S}\left(i \mathfrak{a}_{P q}^{*}\right) \otimes \mathcal{A}_{2, P}\right)^{W\left(\mathfrak{a}_{P q}\right)}$ was asserted in Corollary 22.2 For the converse inclusion, let $\varphi \in\left(\mathcal{S}\left(i \mathfrak{a}_{P \mathbf{q}}^{*}\right) \otimes \mathcal{A}_{2, P}\right)^{W\left(\mathfrak{a}_{P \mathrm{q}}\right)}$. Then

$$
\varphi=\mathrm{P}_{W\left(\mathfrak{a}_{P q}\right)} \varphi=\mathcal{F}_{P}\left(\left[W: W_{P}^{*}\right] \mathcal{J}_{P} \varphi\right) \in \operatorname{im}\left(\mathcal{F}_{P}\right),
$$

in view of Theorem 22.3 (c).

\section{The spherical Plancherel theorem}

In this final section we establish the Plancherel theorem for functions from $\mathcal{C}(\mathrm{X}: \tau)$ and $L^{2}(\mathrm{X}: \tau)$. If $P \in \mathcal{P}_{\sigma}$, then by $\mathcal{C}_{P}(\mathrm{X}: \tau)$ we denote the image of the operator $\mathcal{J}_{P}$ in $\mathcal{C}(\mathrm{X}: \tau)$.

Theorem 23.1 Let $P \in \mathcal{P}_{\sigma}$.

(a) The space $\mathcal{C}_{P}(\mathrm{X}: \tau)$ is closed in $\mathcal{C}(\mathrm{X}: \tau)$ and depends on $P \in \mathcal{P}_{\sigma}$ through its class in $\mathcal{P}_{\sigma} / \sim$.

(b) The spaces $\operatorname{ker} \mathcal{F}_{P}$ and $\mathcal{C}_{P}(\mathrm{X}: \tau)$ are each other's orthocomplement in $\mathcal{C}(\mathrm{X}: \tau)$.

(c) The space $\mathcal{C}(\mathrm{X}: \tau)$ admits the following finite direct sum decomposition

$$
\mathcal{C}(\mathrm{X}: \tau)=\bigoplus_{[R] \in \mathcal{P}_{\sigma} / \sim} \mathcal{C}_{R}(\mathrm{X}: \tau)
$$

The summands are mutually orthogonal with respect to the $L^{2}$-inner product on $\mathcal{C}(\mathrm{X}: \tau)$. 
(d) For each $P \in \mathcal{P}_{\sigma}$, the operator $\left[W: W_{P}^{*}\right] \mathcal{J}_{P} \circ \mathcal{F}_{P}$ is the projection onto $\mathcal{C}_{P}(\mathrm{X}: \tau)$ along the remaining summands in the above direct sum.

Remark 23.2 For the case of the group this result, together with Propositions 22.10 and 22.11 is a consequence of Harish-Chandra's Plancherel theorem for $K$-finite Schwartz functions, see Remark 13.9 and 32, §27. See also [1, Ch. III, $\S 1$, for a review of the Plancherel theorem for spherical Schwartz functions.

For general symmetric spaces, the result, together with Propositions 22.10 and 22.11] is equivalent to Thm. 2 in Delorme's paper [24, in view of Remark 13.10. See also Remark 21.7.

Proof: If $Q \in \mathcal{P}_{\sigma}, Q \nsim P$, then, by Theorem 22.3 (a),

$$
\mathcal{F}_{Q}=0 \quad \text { on } \quad \mathcal{C}_{P}(\mathrm{X}: \tau) .
$$

In view of Theorem 21.6 this implies that

$$
\left[W: W_{P}^{*}\right] \mathcal{J}_{P} \mathcal{F}_{P}=I \quad \text { on } \quad \mathcal{C}_{P}(\mathrm{X}: \tau) .
$$

We infer that $\mathcal{C}_{P}(\mathrm{X}: \tau)=\operatorname{im}\left(\mathcal{J}_{P} \mathcal{F}_{P}\right)$. By Remark 21.5 it follows that $\mathcal{C}_{P}(\mathrm{X}: \tau)$ depends on $P$ through its class in $\mathcal{P}_{\sigma} / \sim$. This establishes the second assertion of (a). From Theorem 21.6 we see that $\mathcal{C}(\mathrm{X}: \tau)$ is the vector sum of the spaces $\mathcal{C}_{R}(\mathrm{X}: \tau)$, for $[R] \in \mathcal{P}_{\sigma} / \sim$. To establish the orthogonality of the summands, let $P, Q \in \mathcal{P}_{\sigma}, P \not Q$, let $f \in \mathcal{C}_{P}(\mathrm{X}: \tau)$ and $\psi \in \mathcal{S}\left(i \mathfrak{a}_{Q \mathrm{q}}^{*}\right) \otimes \mathcal{A}_{2, Q}$. Then $\left\langle f \mid \mathcal{J}_{Q} \psi\right\rangle=\left\langle\mathcal{F}_{Q} f \mid \psi\right\rangle=0$, by Lemma 20.5 and (23.2). This establishes (c). Combining (c) with (23.2) and (23.3), we obtain (d). From (c) it follows that $\mathcal{C}_{P}(\mathrm{X}: \tau)$ is closed, whence (a).

It remains to establish (b). From (23.2) it follows that $\operatorname{ker} \mathcal{F}_{P}$ contains the part $\mathcal{C}_{0}$ of the sum (23.1) consisting of the summands with $R \nsim P$. On the other hand, $\operatorname{ker} \mathcal{F}_{P} \cap \mathcal{C}_{P}(\mathrm{X}: \tau)=0$ by (23.3) and we conclude that $\operatorname{ker} \mathcal{F}_{P}=\mathcal{C}_{0}$. The latter space equals the orthocomplement of $\mathcal{C}_{P}(\mathrm{X}: \tau)$, by the orthogonality of the sum 23.1).

We denote by $L_{P}^{2}(\mathrm{X}: \tau)$ the closure in $L^{2}(\mathrm{X}: \tau)$ of $\mathcal{C}_{P}(\mathrm{X}: \tau)$. This space depends on $P$ through its class in $\mathcal{P}_{\sigma} / \sim$.

Corollary 23.3 The space $L^{2}(\mathrm{X}: \tau)$ admits the following orthogonal direct sum decomposition into closed subspaces

$$
L^{2}(\mathrm{X}: \tau)=\bigoplus_{[P] \in \mathcal{P}_{\sigma} / \sim} L_{P}^{2}(\mathrm{X}: \tau) .
$$

Proof: Since $\mathcal{C}(\mathrm{X}: \tau)$ is dense in $L^{2}(\mathrm{X}: \tau)$, the result is an immediate consequence of Theorem 23.1 (c).

We recall that a continuous linear map between Hilbert spaces $T: \mathcal{H}_{1} \rightarrow \mathcal{H}_{2}$ is called a partial isometry if $T$ is an isometry from $(\operatorname{ker} T)^{\perp}$ into $\mathcal{H}_{2}$. In particular, this means that $\operatorname{im} T$ is a closed subspace of $\mathcal{H}_{2}$. 
Theorem 23.4 Let $P \in \mathcal{P}_{\sigma}$.

(a) The operator $\mathcal{F}_{P}$ extends uniquely to a continuous linear map from $L^{2}(\mathrm{X}: \tau)$ to $L^{2}\left(i \mathfrak{a}_{P \mathrm{q}}^{*}\right) \otimes \mathcal{A}_{2, P}$, denoted $\mathcal{F}_{P}$.

(b) The operator $\mathcal{J}_{P}$ extends uniquely to a continuous linear map from $L^{2}\left(i \mathfrak{a}_{P \mathrm{q}}^{*}\right) \otimes$ $\mathcal{A}_{2, P}$ to $L^{2}(\mathrm{X}: \tau)$, denoted $\mathcal{J}_{P}$. This extension is the adjoint of the extended operator $\mathcal{F}_{P}$.

(c) The extended operator $\left[W: W_{P}^{*}\right]^{1 / 2} \mathcal{F}_{P}$ is a partial isometry from $L^{2}(\mathrm{X}: \tau)$ onto the space $\left(L^{2}\left(i \mathfrak{a}_{P q}^{*}\right) \otimes \mathcal{A}_{2, P}\right)^{W\left(\mathfrak{a}_{P q}\right)}$, with kernel equal to the orthocomplement of $L_{P}^{2}(\mathrm{X}: \tau)$.

(d) The extended operator $\left[W: W_{P}^{*}\right]^{1 / 2} \mathcal{J}_{P}$ is a partial isometry from $L^{2}\left(i \mathfrak{a}_{P q}^{*}\right) \otimes$ $\mathcal{A}_{2, P}$ onto $L_{P}^{2}(\mathrm{X}: \tau)$ with kernel equal to the orthocomplement of $\left(L^{2}\left(i \mathfrak{a}_{P \mathrm{q}}^{*}\right) \otimes\right.$ $\left.\mathcal{A}_{2, P}\right)^{W\left(\mathfrak{a}_{P \mathrm{q}}\right)}$.

(e) Let $\mathbf{P}_{\sigma} \subset \mathcal{P}_{\sigma}$ be a choice of representatives for the classes in $\mathcal{P}_{\sigma} / \sim$. Then

$$
I=\sum_{R \in \mathbf{P}_{\sigma}}\left[W: W_{R}^{*}\right] \mathcal{J}_{R} \mathcal{F}_{R} \quad \text { on } \quad L^{2}(\mathrm{X}: \tau)
$$

Remark 23.5 In view of Remark 13.10 this result corresponds to part (iv) of Theorem 2 in 24]. See also Remark 23.2.

Proof: Fix $\mathbf{P}_{\sigma}$ as in (e). Let $f \in \mathcal{C}(\mathrm{X}: \tau)$. Then it follows from Theorem 23.1 (c) and (d), combined with Lemma 20.5 that

$$
\|f\|^{2}=\langle f \mid f\rangle=\sum_{R \in \mathbf{P}_{\sigma}}\left[W: W_{R}^{*}\right]\left\langle f \mid \mathcal{J}_{R} \mathcal{F}_{R} f\right\rangle=\sum_{R \in \mathbf{P}_{\sigma}}\left[W: W_{R}^{*}\right]\left\|\mathcal{F}_{R} f\right\|^{2} .
$$

In particular, this equation holds for a choice of $\mathbf{P}_{\sigma}$ with $P \in \mathbf{P}_{\sigma}$. It follows that $\mathcal{F}_{P}$ is continuous with respect to the $L^{2}$-topologies. By density of $\mathcal{C}(\mathrm{X}: \tau)$ in $L^{2}(\mathrm{X}: \tau)$, it follows that $\mathcal{F}_{P}$ has a unique continuous linear extension $L^{2}(\mathrm{X}: \tau) \rightarrow L^{2}\left(i \mathfrak{a}_{P \mathrm{q}}^{*}\right) \otimes \mathcal{A}_{2, P}$. Hence (a).

Since $\mathcal{J}_{P}$ is the adjoint of $\mathcal{F}_{P}$ with respect to the $L^{2}$-inner products on the Schwartz spaces, it follows that the adjoint of the extension of $\mathcal{F}_{P}$ is a continuous linear extension of $\mathcal{J}_{P}$ to a continuous linear operator from $L^{2}\left(i \mathfrak{a}_{P q}^{*}\right) \otimes \mathcal{A}_{2, P}$ to $L^{2}(\mathrm{X}: \tau)$. This extension is unique by density of $\mathcal{S}\left(i \mathfrak{a}_{P \mathrm{q}}^{*}\right)$ in $L^{2}\left(i \mathfrak{a}_{P \mathrm{q}}^{*}\right)$. This proves (b).

By continuity and density, the formula in (e) follows from Theorem 21.6. From Theorem 23.1 (b) and (c) it follows that, for $R \in \mathbf{P}_{\sigma}$ with $R \nsim P$, $\mathcal{F}_{P}=0$ on $\mathcal{C}_{R}(\mathrm{X}: \tau)$, hence on $L_{R}^{2}(\mathrm{X}:, \tau)$, by continuity and density. Put $\widetilde{\mathcal{F}}_{P}:=$ $\left[W: W_{P}^{*}\right]^{1 / 2} \mathcal{F}_{P}$ and $\widetilde{\mathcal{J}}_{P}:=\left[W: W_{P}^{*}\right]^{1 / 2} \mathcal{J}_{P}$. Using (e) we infer that ker $\widetilde{\mathcal{F}}_{P}=$ $L_{P}^{2}(\mathrm{X}: \tau)^{\perp}$ and that $\widetilde{\mathcal{J}}_{P} \circ \widetilde{\mathcal{F}}_{P}$ is the orthogonal projection from $L^{2}(\mathrm{X}: \tau)$ onto $L_{P}^{2}(\mathrm{X}: \tau)$. Since $\widetilde{\mathcal{J}}_{P}=\widetilde{\mathcal{F}}_{P}^{*}$, it follows that $\widetilde{\mathcal{F}}_{P}$ is isometric from $L_{P}^{2}(\mathrm{X}: \tau)$ onto $\operatorname{im} \widetilde{\mathcal{F}}_{P}$ and that $\widetilde{\mathcal{J}}_{P}$ is isometric from $\operatorname{im} \widetilde{\mathcal{F}}_{P}$ onto $L_{P}^{2}(\mathrm{X}: \tau)$. It follows from 
Theorem 22.3 and continuity and density that $\widetilde{\mathcal{F}}_{P} \circ \widetilde{\mathcal{J}}_{P}=\mathrm{P}_{W\left(\mathfrak{a}_{P q}\right)}$ on $L^{2}\left(i \mathfrak{a}_{P \mathrm{q}}^{*}\right) \otimes$ $\mathcal{A}_{2, P}$. Hence,

$$
\operatorname{im}\left(\widetilde{\mathcal{F}}_{P}\right)=\widetilde{\mathcal{F}}_{P}\left(L_{P}^{2}(\mathrm{X}: \tau)\right)=\operatorname{im}\left(\mathrm{P}_{W\left(\mathfrak{a}_{P q}\right)}\right)=\left(L^{2}\left(i \mathfrak{a}_{P \mathrm{q}}^{*}\right) \otimes \mathcal{A}_{2, P}\right)^{W\left(\mathfrak{a}_{P q}\right)}
$$

and (c) follows. Finally, (d) follows from (c) by taking adjoints.

\section{References}

[1] J. Arthur, A Paley-Wiener theorem for real reductive groups. Acta Math. 150 (1983), 1-89.

[2] E. P. van den Ban, Invariant differential operators on semisimple symmetric spaces and finite multiplicities in a Plancherel formula. Ark. för Mat. 25 (1987), 175-187.

[3] E. P. van den Ban, Asymptotic behaviour of matrix coefficients related to reductive symmetric spaces. Indag. Math. 49, 1987, 225-249.

[4] E. P. van den Ban, The principal series for a reductive symmetric space, I. $H$-fixed distribution vectors. Ann. scient. Éc. Norm. Sup. 21 (1988), $359-412$.

[5] E. P. van den Ban, The principal series for a reductive symmetric space II. Eisenstein integrals. J. Funct. Anal. 109 (1992), 331-441.

[6] E. P. van den Ban, The action of intertwining operators on spherical vectors in the minimal principal series of a reductive symmetric space. Indag. Math. 145 (1997), 317-347.

[7] E. P. van den Ban, J. Carmona and P. Delorme, Paquets d' ondes dans l'espace de Schwartz d'un espace symétrique réductif. J. Funct. Anal. 139 (1996), 225-243.

[8] E. P. van den Ban and H. Schlichtkrull, Asymptotic expansions and boundary values of eigenfunctions on Riemannian symmetric spaces. J. reine angew. Math. 380 (1987), 108-165.

[9] E. P. van den Ban and H. Schlichtkrull, Fourier transforms on a semisimple symmetric space. Invent. Math. 130 (1997), 517-574.

[10] E. P. van den Ban and H. Schlichtkrull, The most continuous part of the Plancherel decomposition for a reductive symmetric space. Annals Math. 145 (1997), 267-364.

[11] E. P. van den Ban and H. Schlichtkrull, A residue calculus for root systems. Compositio Math. 123 (2000), 27-72. 
[12] E. P. van den Ban and H. Schlichtkrull, Fourier inversion on a reductive symmetric space. Acta Math. 182 (1999), 25-85.

[13] E. P. van den Ban and H. Schlichtkrull, Harmonic analysis on reductive symmetric spaces. pp. 565-582 in: European Congress of Math., Barcelona 2000, Vol I, C. Casacuberta, e.a., eds. Progress in Math. 201, Birkhäuser Verlag, 2001.

[14] E. P. van den Ban and H. Schlichtkrull, Analytic families of eigenfunctions on a reductive symmetric space. Represent. Theory 5 (2001), 615-712.

[15] E. P. van den Ban and H. Schlichtkrull, The Plancherel decomposition for a reductive symmetric space, II. Representation theory. Preprint 2001, arXiv:math.RT/0111304

[16] E. P. van den Ban and H. Schlichtkrull, A Paley-Wiener theorem for reductive symmetric spaces. Preprint 2003, arXiv:math.RT/0302232

[17] J. Carmona, Terme constant des fonctions tempérées sur un espace symétrique réductif. J. reine angew. Math. 491 (1997), 17-63.

[18] J. Carmona and P. Delorme, Base méromorphe de vecteurs distributions $\mathrm{H}$-invariants pour les séries principales généralisées d'espaces symétriques réductifs: Equation fonctionelle. J. Funct. Anal. 122 (1994), 152-221.

[19] J. Carmona and P. Delorme, Transformation de Fourier sur l'espace de Schwartz d'un espace symétrique réductif. Invent. math. 134 (1998), 5999.

[20] W. Casselman, Canonical extensions of Harish-Chandra modules to representations of $G$. Canad. J. Math. 41 (1989), 385-438.

[21] W. Casselman and D. Miličić, Asymptotic behavior of matrix coefficients of admissible representations. Duke Math. J. 49 (1982), 869-930.

[22] P. Delorme, Intégrales d'Eisenstein pour les espaces symétriques réductifs: tempérance, majorations. Petite matrice B. J. Funct. Anal. 136 (1994), $422-509$.

[23] P. Delorme, Troncature pour les espaces symétriques réductifs. Acta Math. 179 (1997), 41-77.

[24] P. Delorme, Formule de Plancherel pour les espaces symétriques réductifs. Annals of Math. 147 (1998), 417-452.

[25] M. Flensted-Jensen, Discrete series for semisimple symmetric spaces. Annals Math. 111 (1980), 253-311.

[26] P. Harinck, Fonctions orbitales sur $G_{\mathbb{C}} / G_{\mathbb{R}}$. Formule d'inversion des intégrales orbitales et formule de Plancherel. J. Funct. Anal. 153 (1998), 52-107. 
[27] Harish-Chandra, Spherical functions on a semisimple Lie group, I, II. Amer. J. Math. 80 (1958), 241-310, 553-613. Also: Collected papers, Vol 2, pp. 409-539. Springer-Verlag, New York, 1984.

[28] Harish-Chandra, Discrete series for semisimple Lie groups. II Explicit determination of the characters. Acta Math. 116 (1966), 1-111. Also: Collected papers, Vol 3, pp. 537-647. Springer-Verlag, New York, 1984.

[29] Harish-Chandra, On the theory of the Eisenstein integral. Lecture Notes in Math. 266, 123-149, Springer-Verlag, New York, 1972. Also: Collected papers, Vol 4, pp. 47-73, Springer-Verlag, New York, 1984.

[30] Harish-Chandra, Harmonic analysis on real reductive groups I. The theory of the constant term. J. Funct. Anal. 19 (1975), 104-204. Also: Collected papers, Vol 4, pp. 102-202, Springer-Verlag, New York, 1984.

[31] Harish-Chandra, Harmonic analysis on real reductive groups II. Wave packets in the Schwartz space. Invent. math. 36 (1976), 1-55. Also: Collected papers, Vol 4, pp. 203-257, Springer-Verlag, New York, 1984.

[32] Harish-Chandra, Harmonic analysis on real reductive groups III. The Maass-Selberg relations and the Plancherel formula. Annals of Math. 104 (1976), 117-201. Also: Collected papers, Vol 4, pp. 259-343, SpringerVerlag, New York, 1984.

[33] G. Heckman and H. Schlichtkrull, Harmonic analysis and special functions on symmetric spaces. Perspectives in Math. 16, Academic Press, Inc., San Diego, 1994.

[34] S. Helgason, The surjectivity of invariant differential operators on symmetric spaces I. Annals of Math. 98 (1973), 451-479.

[35] S. Helgason, Groups and geometric analysis. Integral geometry, invariant differential operators, and spherical functions. Math. Surveys and Monographs, 83. AMS, Providence, RI, 2000.

[36] A. W. Knapp and E. Stein, Intertwining operators for semisimple Lie groups, II. Invent. Math. 60 (1980), 9-84.

[37] R. P. Langlands, On the functional equations satisfied by Eisenstein series. Springer Lecture Notes 544, Springer-Verlag, Berlin, 1976.

[38] O. Loos, Symmetric spaces 1. W.A. Benjamin, 1969.

[39] T. Oshima, A realization of semisimple symmetric spaces and construction of boundary value maps. Adv. Studies in Pure Math. 14 (1988), 603-650.

[40] T. Oshima and T. Matsuki, A description of discrete series for semisimple symmetric spaces. Adv. Stud. Pure Math. 4 (1984), 331-390. 
[41] V. S. Varadarajan, Harmonic analysis on real reductive groups. Lect. Notes in Math. 576, Springer-Verlag, Berlin, 1977.

[42] N. R. Wallach, Real Reductive Groups I. Academic Press, Inc., San Diego, 1988.

[43] N. R. Wallach, Real Reductive Groups II. Academic Press, Inc., San Diego, 1992.

E.P. van den Ban

Mathematisch Instituut

Universiteit Utrecht

PO Box 80010

3508 TA Utrecht

Netherlands

E-mail: ban@math.uu.nl
H. Schlichtkrull

Matematisk Institut

Københavns Universitet

Universitetsparken 5

2100 København $\varnothing$

Denmark

E-mail: schlicht@math.ku.dk 